Attachment and mentalization as predictors of outcome in family therapy for adolescent anorexia nervosa

Tom Jewell

PhD Thesis, 2021

Department of Child and Adolescent Psychiatry,

Institute of Psychiatry, Psychology and Neuroscience,

King's College London 


\begin{abstract}
Family therapy for adolescent anorexia nervosa (FT-AN) is the first line treatment for the disorder, but little is known about who the treatment works for, or how it works. This thesis investigates the potential value of the constructs of attachment and mentalization as predictors of outcome in family therapy for adolescent AN.
\end{abstract}

The thesis presents a systematic review of attachment and mentalization and their association with child and adolescent eating pathology in which 22 relevant studies were found. In keeping with the evidence base for adults with eating disorders, both attachment and mentalization were found to be correlates of eating pathology in childhood and adolescence. However, evidence for these constructs in the process of treatment in child and adolescent eating disorders is scarce. In a second systematic review, the psychometric properties of attachment measures in middle childhood and adolescence were investigated across 53 studies. The overall conclusion of the study is that there is a lack of evidence of adequate psychometric properties for attachment measures in this age group.

In the main study, attachment and mentalization were investigated as predictors of outcome in a sample of adolescents and their parents $(n=192)$ receiving FT-AN across three specialist outpatient eating disorder services. Mentalization, but not attachment, was found to predict poor clinical outcome at nine months after starting of treatment, with the strongest predictive effect being excessive certainty about mental states as assessed in parents. Therapeutic alliance scores at one month were predictive of outcome but did not play a mediating role in relation to baseline attachment or mentalization. 
In a further empirical study, definitions of eating disorder recovery were investigated through a qualitative analysis of comments on recovery-focused social media forums. Two superordinate themes emerged: firstly, recovery was defined as encompassing broad psychosocial domains of wellbeing; secondly, recovery was understood to be an ongoing process. Social connectedness to others was regarded as important both to the process and definition of eating disorder recovery.

The final chapter of the thesis integrates findings from across the studies with emerging theoretical developments in the fields of attachment and mentalization. It is argued that attachment and mentalization represent worthwhile constructs for further FT-AN research, with a particular emphasis on the need to better understand the mechanisms by which excessive mental state certainty might lead to poor clinical outcomes. 


\section{Table of Contents}

Abstract $\quad$ ii

Table of Contents $\quad$ iv

List of Tables $\quad x$

List of Figures $\quad$ xi

Statement of Authorship $\quad$ xii

Publications resulting from work in the thesis $\quad$ xiv

Acknowledgements $\quad$ xvi

Chapter 1 - Background 1

1.1. Introduction 1

1.1.1 Anorexia nervosa 2

1.1.2 Hilde Bruch's views on family influences on anorexia nervosa 2

1.1.3 Early roots of family therapy for eating disorders 5

$\begin{array}{ll}\text { 1.1.4 The Maudsley model } & 7\end{array}$

1.1.5 Complex interventions revisited 10

1.1.6 The need for new concepts and theory in FT-AN 13

1.1.7 Attachment and mentalization 15

1.1.8 Attachment, mentalization and eating disorders 17

1.1.9 Attachment, mentalization and family therapy 17

1.2 Summary and outline of this thesis 19

Chapter 2 - Family therapy for child and adolescent eating disorders: A critical review 23

2.1 Abstract $\quad 24$

2.2 Introduction $\quad 25$

2.3 Description of the treatment 27

2.3.1 Family therapy for Anorexia Nervosa (FT-AN) 27

2.3.2 Multi-Family Therapy for Anorexia Nervosa (MFT-AN) 30

2.3.3 Family therapy for bulimia nervosa (FT-BN) 31

2.3.4 Multi-family therapy for bulimia nervosa (MFT-BN) 31

2.4 Efficacy research $\quad 32$

2.4.1 Relative efficacy of FT-AN 32 
2.4.3 Efficacy trials comparing different forms of FT-AN 36

2.4.4 Efficacy of FT-AN compared with generic family therapy approaches 37

2.4.5 Efficacy of MFT-AN and MFT-BN 39

2.5 Findings on implementation and service context 40

2.5.1 Implementation Studies $\quad 40$

2.5.2 Service context as a possible common factor in the treatment of eating disorders $\quad 40$

2.6 Predictors, moderators and mediators of outcome $\quad 42$

2.6.1 Predictors of outcome $\quad 42$

2.6.2 Moderators $\quad 42$

2.6.3 Potential mediators of treatment 45

2.6.4 Therapeutic alliance $\quad 47$

$\begin{array}{ll}2.7 \text { Discussion } & 49\end{array}$

2.8 Conclusion $\quad 51$

2.9 Updated literature review $\quad 53$

2.9.1 Randomised controlled trials $\quad 53$

2.9.2 Reviews $\quad 55$

2.9.3 Empirical studies $\quad 59$

2.9.4 Summary of the updated literature 61

Chapter 3 - Attachment and mentalization and their association with child and adolescent $\begin{array}{ll}\text { eating pathology: A systematic review } & 63\end{array}$

$\begin{array}{ll}3.1 \text { Abstract } & 64\end{array}$

$\begin{array}{ll}3.2 \text { Introduction } & 65\end{array}$

3.2.1 Attachment 66

3.2.2 Mentalization $\quad 68$

$\begin{array}{ll}3.3 \text { Rationale for this review } & 70\end{array}$

$\begin{array}{ll}3.4 \text { Method } & 71\end{array}$

3.4.1 Assessment of bias

$\begin{array}{ll}3.5 \text { Results } & 73\end{array}$

3.5.1 Risk of bias within studies $\quad 73$

3.5.2 Cross-sectional studies of attachment and eating pathology 86

3.5.3 Cross-sectional studies involving tests of mediation $\quad 88$

3.5.4 Prospective studies of attachment and eating pathology $\quad 89$

3.5.5 Studies of mentalization and eating pathology 93

$\begin{array}{ll}3.6 \text { Discussion } & 98\end{array}$ 
3.6.1 Implications for theory

$\begin{array}{ll}\text { 3.6.2 Implications for research } & 102\end{array}$

$\begin{array}{ll}3.7 \text { Limitations } & 104\end{array}$

$\begin{array}{ll}3.8 \text { Conclusion } & 104\end{array}$

3.9 Updated literature review $\quad 106$

3.9.1 Studies of attachment and eating pathology 106

3.9.2 Reviews of attachment and eating pathology 106

3.9.3 Community studies of attachment and eating pathology 108

3.9.4 Studies of attachment in adolescent eating disorder samples 112

$\begin{array}{ll}\text { 3.9.5 Studies of mentalization and eating pathology } & 114\end{array}$

$\begin{array}{ll}\text { 3.9.6 Summary of the updated literature } & 117\end{array}$

Chapter 4 - Attachment measures in middle childhood and adolescence: A systematic review

$\begin{array}{ll}\text { of measurement properties } & 118\end{array}$

$\begin{array}{ll}4.1 \text { Abstract } & 119\end{array}$

$\begin{array}{ll}\text { 4.1.1 Abbreviations } & 120\end{array}$

$\begin{array}{ll}4.2 \text { Introduction } & 121\end{array}$

4.2.1 Measuring attachment in infancy and adulthood 122

4.2.2 Measuring attachment in middle childhood and adolescence 123

4.2.3 Rationale for this review $\quad 125$

$\begin{array}{ll}\text { 4.2.4 Objective } & 125\end{array}$

$\begin{array}{ll}4.3 \text { Method } & 125\end{array}$

$\begin{array}{ll}\text { 4.3.1 Literature search } & 125\end{array}$

4.3.2 Data extraction 126

4.3.3 Assessment of measurement properties 126

$\begin{array}{ll}\text { 4.3.4 Data synthesis } & 127\end{array}$

4.4 Results 139

4.4.1 Internal consistency 139

$\begin{array}{ll}\text { 4.4.2 Test-retest reliability } & 139\end{array}$

4.4.3 Inter-rater reliability (interview and projective measures) 141

$\begin{array}{ll}\text { 4.4.4 Content validity } & 141\end{array}$

$\begin{array}{ll}\text { 4.4.5 Structural validity } & 141\end{array}$

4.4.6 Hypotheses testing 142

4.4.7 Cross-cultural validity 143

4.4.8 Criterion validity 143

4.4.9 Synthesis of results 143 
4.5.1 What is the most valid and reliable approach to assessing attachment in middle childhood and adolescence?

4.5.2 Is attachment distributed categorically or continuously? 148

4.5.3 Implications for research 149

4.5.4 Implications for clinical practice 151

4.5.5 Limitations 152

$\begin{array}{ll}4.6 \text { Conclusion } & 153\end{array}$

Chapter 5 - Attachment and mentalization as predictors of outcome in family therapy for

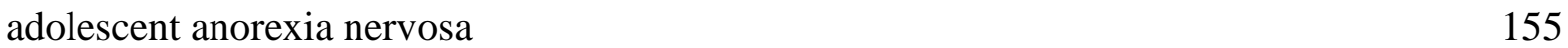

5.1. Abstract 156

5.2. Introduction $\quad 157$

5.2.1 Proposed links between attachment, mentalization and treatment outcome $\quad 159$

5.2.2 The potential role of emotional regulation $\quad 160$

5.2.3 Hypotheses: $\quad 161$

5.2.4 Secondary analysis 163

$\begin{array}{ll}\text { 5.3. Methods } & 163\end{array}$

5.3.1 Data collection 164

5.3.2 Outcomes 164

5.3.3 Adolescent exposure variables (T1): 165

5.3.4 Parent exposure variables (T1) 166

5.3.5 Adolescent and parent mediator variables (T2) 167

5.3.6 Baseline covariates 167

5.3.7 Baseline measures of comorbidity 168

$\begin{array}{ll}\text { 5.3.8 Study registration } & 168\end{array}$

5.3.9 Ethics 168

5.3.10 Sample size 169

5.3.11 Internal reliability 169

5.3.12 Public and Patient Involvement (PPI) 169

$\begin{array}{ll}\text { 5.3.13 Analysis plan } & 170\end{array}$

5.3.14 Missing data 171

$\begin{array}{ll}\text { 5.3.15 Multiple imputation } & 171\end{array}$

5.4 Results 173

$\begin{array}{ll}\text { 5.4.1 Demographics } & 173\end{array}$

$\begin{array}{ll}\text { 5.4.2 Descriptive statistics } & 177\end{array}$ 
5.4.3 Correlations between baseline variables

$\begin{array}{ll}\text { 5.4.5 Prediction of outcome from baseline covariates } & 180\end{array}$

5.4.6 Associations between exposure, mediator and outcome variables $\quad 180$

5.4.7 Prediction of outcome from baseline exposure variables $\quad 180$

5.4.8 Prediction of outcome by alliance (hypothesised mediator) 180

5.4.9 Prediction of alliance by baseline exposure variables 181

5.4.10 Mediation analysis $\quad 181$

5.4.11 Secondary analysis - prediction of percentage median Body Mass Index at 9 months 182

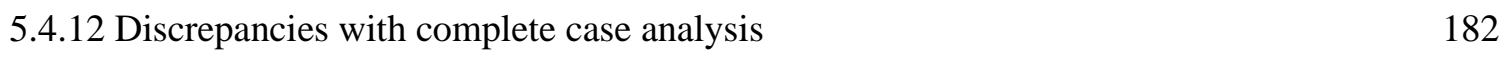

$\begin{array}{ll}5.5 \text { Discussion } & 184\end{array}$

$\begin{array}{ll}\text { 5.5.1. Limitations } & 187\end{array}$

$\begin{array}{ll}\text { 5.6. Conclusion } & 188\end{array}$

Chapter 6 - Recovery as a process: Exploring definitions of recovery in the context of eating$\begin{array}{ll}\text { disorder-related social media forums } & 189\end{array}$

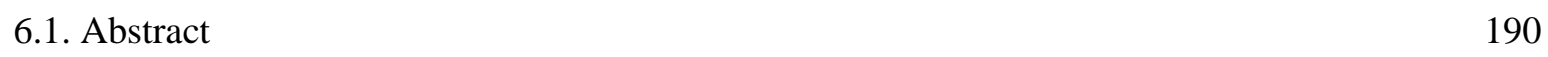

6.2. Introduction 191

6.2.1 Distinguishing remission and recovery 191

6.2.2 Defining recovery and remission in adolescent anorexia nervosa 193

6.2.3 Perspectives on recovery from persons with lived experience 194

6.2.4 Social media and eating disorder recovery 194

$\begin{array}{ll}6.3 \text { Method } & 195\end{array}$

6.3.1 Corpus Selection and Data Extraction 195

6.3.2 Data Analysis 196

6.4 Results 196

6.4.1 User Characteristics 196

6.4.2 Data Corpus Characteristics 197

$\begin{array}{ll}\text { 6.4.3 Themes } & 197\end{array}$

$\begin{array}{ll}\text { 6.4.4 Recovery as a process } & 199\end{array}$

6.4.5 Psychosocial factors 200

$\begin{array}{ll}6.5 \text { Discussion } & 201\end{array}$

6.5.1 Attachment, affiliation and eating disorder recovery 202

6.5.2 Eating disorder recovery - universal or unique? 204

6.5.3 Implications for service provision 205

6.5.4 Limitations 205

6.6 Conclusion and Future Directions 206 
7.1. Introduction and overview of the thesis 207

7.2. Attachment and mentalizing - trait or state? 209

7.3. Common and specific factors in FT-AN 211

7.4. New developments in attachment and mentalization 215

7.5. Revisiting theory about mechanisms of change in FT-AN 216

$\begin{array}{ll}\text { 7.5.1 Excessive certainty, rigidity and anorexia nervosa } & 218\end{array}$

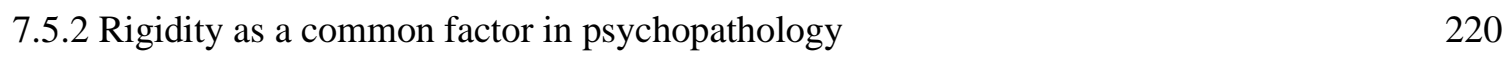

$\begin{array}{ll}\text { 7.5.3 Social relationships and recovery } & 221\end{array}$

7.5.4 Summary of proposed mechanisms of change in FT-AN 223

7.6 Recommendations for future research 223

$\begin{array}{ll}\text { 7.6.1 Psychometric work } & 223\end{array}$

7.6.2 Better characterisation of families at risk of poor outcome 224

7.6.3 Prospective observational studies of treatment outcome in FT-AN 225

$\begin{array}{ll}\text { 7.6.4 Experimental studies } & 226\end{array}$

$\begin{array}{ll}\text { 7.7. Conclusion } & 227\end{array}$

$\begin{array}{ll}\text { References } & 229\end{array}$

$\begin{array}{ll}\text { Appendices } & 288\end{array}$

Appendix A: Characteristics of included studies - Observer-rated measures 289

Appendix B: Characteristics of included studies - Self-report measures 291

Appendix C: Methodological quality and adequacy of psychometric properties - Observer-rated measures 293

Appendix D: Methodological quality and adequacy of psychometric properties - Self-report measures

Appendix E: COSMIN criteria for evidence synthesis 297

Appendix F: Reference list of studies included in the review 298

Appendix G: Parent Exposure Variables by parental gender 306

Appendix H: Adolescent self-reported baseline and exposure variables by gender 308

Appendix I: Means and Standard Deviations of Baseline Variables and Self-Report Measures by Site

Appendix J: Associations between parent exposure, mediator and outcome variables 312

Appendix K: Associations between adolescent exposure, mediator and outcome variables $\quad 314$ 


\section{List of Tables}

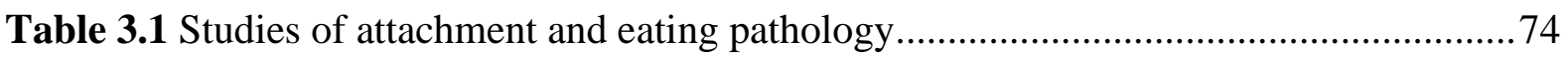

Table 3.2 Studies of mentalization and eating pathology in children and adolescents 80

Table 3.3 Assessment of risk of bias within individual studies of attachment and eating pathology

Table 3.4 Assessment of risk of bias within individual studies of attachment and eating pathology

Table 4.1 Characteristics, strengths and weaknesses of observer-rated measures

Table 4.2 Characteristics, strengths and weaknesses of self-report measures

Table 4.3 COSMIN Criteria for adequacy of measurement properties.

Table 4.4 Synthesis of psychometric properties and level of evidence for observer-rated measures

Table 4.5 Synthesis of psychometric properties and level of evidence for self - report measures

Table 5.1 Demographic characteristics of the sample.

Table 5.2 Range, Means, Standard Deviations and Internal Reliability of Self-Report

Measures

Table 5.3 Correlations between key baseline and exposure variables

Table 6.1 Eating-disorder subreddit themes 198 


\section{List of Figures}

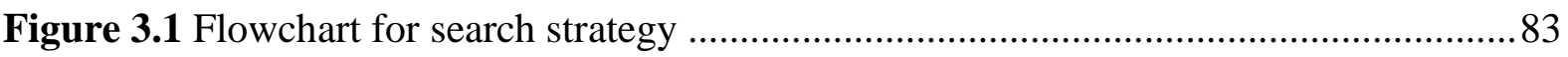

Figure 4.1 Flowchart for search strategy .............................................................. 140

Figure 5.1 Conceptual diagram showing hypothesised model. ......................................... 162

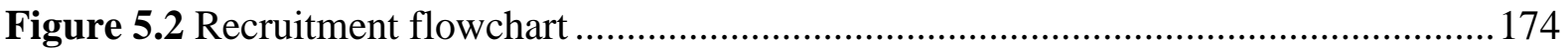

Figure 5.3 Mediation model showing associations between parental exposure, parental mediator and outcome. 


\section{Statement of Authorship}

All work presented in the thesis is my own, except where acknowledged in the text. All investigations were conceived of and carried out by me, as first author, in collaboration with colleagues included in the author lists that precede each chapter. Chapter-specific author contribution summaries are included below.

Chapter 2: TJ, EB, CS, MS and IE conceived the manuscript; TJ wrote the manuscript; all authors critically reviewed the manuscript and provided comments during submission and revision.

Chapter 3: TJ, IE and PF conceived the systematic review; TJ and TG ran the searches; TJ assessed study eligibility; TJ and HC assessed risk of bias; TJ wrote the manuscript; all authors critically reviewed the manuscript and provided comments during submission and revision.

Chapter 4: TJ, IE and PF wrote the protocol for the systematic review; TJ, TG and KW conducted literature searches; TJ, TG, KS, EC and KW rated the included studies; TJ wrote the manuscript; all authors critically reviewed the manuscript and provided comments during submission and revision.

Chapter 5: TJ, IE and PF conceived the study; TJ collected the data entirely from Site 1, and largely collected the data from participants in Sites 2 and 3, where there was also support from local staff under the supervision of LS and AE, who were Principal Investigators at their sites; TJ ran the statistical analyses under the guidance of $\mathrm{MH}$, who was the statistical advisor for the study; TJ wrote the chapter which 
contains elements of the preprint manuscript; all authors critically reviewed the preprint manuscript.

Chapter 6: TJ, BB and UF conceived the study; TJ and UF conducted the analysis; TJ wrote the thesis chapter which contains elements of the published manuscript including Method and Results; TJ, BB and UF co-authored the published manuscript. 


\section{Publications resulting from work in the thesis}

Chapters 2, 3, 4 and 6 are based on published papers with the following details:

Chapter 2: Jewell, T., Blessitt, E., Stewart, C., Simic, M. and Eisler, I. (2016). Family Therapy for Child and Adolescent Eating Disorders: A Critical Review. Family Process, 55(3), 577-594

Chapter 3: Jewell, T., Collyer, H., Gardner, T., Tchanturia, K., Fonagy, P., Simic, M. and Eisler, I. (2016). Attachment and mentalization and their association with child and adolescent eating pathology: A systematic review. International Journal of Eating Disorders, 49(4), 354-373.

Chapter 4: Jewell, T., Gardner, T., Susi, K., Watchorn, K., Coopey, E., Simic, M., Fonagy, P. and Eisler, I. (2019). Attachment measures in middle childhood and adolescence: a systematic review of measurement properties. Clinical Psychology Review, 68, 71-82.

Chapter 6: Bohrer, B., Foye, U. and Jewell, T. (2020). Recovery as defined by users of eating-disorder-related social media forums. International Journal of Eating Disorders, 53(8), 1219-1223. 


\section{Manuscripts under review:}

Chapter 5 is an extended version of the following manuscript which is currently under review:

Jewell, T., Herle, M., Serpell, L., Eivors, A., Simic, M., Fonagy, P., \& Eisler, I. (2020, November 9). Attachment and mentalization as predictors of outcome in family therapy for adolescent anorexia nervosa. https://doi.org/10.31234/osf.io/fgytk

\section{Other publications during the $\mathbf{P h D}$}

Jewell, T. and Lemmens, G. (2018). Multiple Family Therapy: forever promising? Commentary on Gelin et al. (2018) and Cook-Darzens et al. (2018), The evidence base for Multiple Family Therapy in psychiatric and non-psychiatric conditions: a review (parts 1 and 2). Journal of Family Therapy, 40(3), 344-348.

Simic, M., Jewell, T. and Eisler, I. (2020). Beneath the surface of expressed emotion: The clinical relevance of possible mechanisms underlying EE in eating disorders. In D. Le Grange and R. Rienecke (Eds.), Eating Disorders and Expressed Emotion: Integrating Treatment, Intervention, and a Positive Family Environment (pp. 33-57). Hove: Routledge. 


\section{Acknowledgements}

I would like to begin by thanking all the participants who took part in my research at such a difficult and all-consuming time in their lives. I cannot thank you enough, and I hope that the work contained in this thesis contributes at least in some small way to advancing clinical practice in the future.

This thesis would not have been possible without funding from the Clinical Doctoral Research Fellowship scheme of the National Institute of Health Research (CDRF2014-05-024) - I am deeply grateful for the opportunity to do this work. I would also like to express my sincere gratitude to my supervisors, Professor Ivan Eisler and Professor Peter Fonagy, for their support and advice before, during and after the fellowship, and for helping to develop my thinking throughout the last seven years or so.

There are many individuals who contributed to the work contained in this thesis. I would like to thank all my co-authors for their hard work, with a particular shout-out to all the early career researchers who helped me: Karima Susi, Emily Coopey, Tessa Gardner, Kate Watchorn and Hannah Collyer, who endured countless hours in the trenches of the systematic reviews: Una Foye and Britt Bohrer - our work together was a high-point of the fellowship; and finally Moritz Herle - thanks so much for all your help, I learnt so much from working with you. I also want to express a big thank you to Duncan McCaig for the data extraction and early conversations regarding the study presented in Chapter 6. 
Recruiting participants across three NHS sites was a huge undertaking and the main study could never have happened without the support of a huge number of clinicians and assistant psychologists. I would particularly like to thank Alison Eivors and Lucy Serpell for their involvement and support in Leicester and NELFT, and Mima Simic and Catherine Stewart for their support at the Maudsley. I was fortunate to supervise a host of excellent MSc students during the fellowship, and I am grateful to the following for all their help over the years: Ndidiamaka Gordon, Jennifer Danby, Alice Bowen, Rasa Kubiliute, Geraldine Lannon and Georgia Drymonitou.

Finally, I would like to thank my 'buggly' family of Jess, Elliot and Cara for all their patience and support over the course of the PhD. I love you. 


\section{Chapter 1: Background}

\subsection{Introduction}

Family therapy is the treatment with the most evidence of efficacy in the treatment of adolescent anorexia nervosa, but two key questions have received insufficient attention: Who does it work for? And how does it work? This thesis aims to address both questions by investigating the role of attachment and mentalization in the process and outcome of family therapy for adolescent anorexia nervosa (FT-AN) (Eisler et al., 2016a; National Institute for Health and Care Excellence, 2017). In so doing, the thesis will bring to bear new theory and evidence that can inform the development of new treatments, or the adaptation of existing ones.

Effective interventions require sound theory (Pennesi \& Wade, 2016), but the relationship between theory and practice in FT-AN has been complicated by its history. In the influential Medical Research Council model for the development of complex interventions (Craig et al., 2008), the identification and development of theory is a key step in the research cycle. FT-AN's development has been informed by both theory and research (Eisler, 2005; Eisler, Wallis and Dodge, 2016), but the field's progress has been hampered by a lack of testable models (Pennesi and Wade, 2016) and research with adequate power to investigate moderators and mediators of outcome (Hamadi \& Holliday, 2020). This problem is not unique to FT-AN: according to Cuijpers et al. (2019), no common or specific factor meets criteria as an empirically validated working mechanism in psychotherapy. Thus, the task of furthering understanding of change processes in FT-AN is a prodigious one. 
In this chapter we shall introduce anorexia nervosa, FT-AN, attachment and mentalizing and place them in historical context, before outlining the content of the overall thesis. Two main arguments will be advanced in this chapter: firstly, that a knowledge of the history of ideas informing FT-AN is necessary to understand why the treatment has a relatively under-developed empirical and theoretical base with regards to change processes; secondly, that attachment and mentalization constitute promising avenues for FT-AN research and practice.

\section{$\underline{1.1 .1 \text { Anorexia nervosa }}$}

Anorexia nervosa (AN) is a serious psychiatric condition which typically emerges during adolescence, characterised by food restriction, distorted body image and intense fear of weight gain (American Psychiatric Association, 2013). AN is associated with high rates of psychiatric co-morbidity (Salbach-Andrae et al., 2008) and significantly raised mortality rates (Arcelus et al., 2011). Point prevalence of AN in adolescence has been estimated at $0.7 \%$ (Mitchison et al., 2020), whilst incidence has been estimated at 14 per 100000 (Petkova et al., 2019). Today the disorder is understood as having multiple risk factors across a broad range of domains including biological, psychological and behavioural domains (see Jacobi et al., 2004 and Zipfel et al., 2015 for reviews). However, over the past 300 years there have been various accounts of the aetiology of AN (Silverman, 1997).

\subsubsection{Hilde Bruch's views on family influences on anorexia nervosa}

In the decades following the Second World War, psychoanalytic accounts of aetiology dominated the field, and dozens of such theories were proposed (Kog \& Vandereycken, 1989). In terms of impact and lasting influence, the ideas developed by Hilde Bruch $(1973,1978)$, a psychiatrist and psychoanalyst, are regarded as some of the most influential in the eating disorder field over recent decades (Skarderud, 
2009; Treasure and Cardi, 2017) and are of particular relevance to the constructs investigated in this thesis.

Bruch claimed that the "development of anorexia nervosa is so closely related to abnormal patterns of family interaction that successful treatment must always involve resolution of the underlying family problems, which may not be identifiable as open conflicts: on the contrary, quite often excessive closeness and overintense involvements lie at the roots" (Bruch, 1978, p.106). Bruch hypothesized that the early relationship between infant and mother had profound and lasting effects on the mental, emotional and physiologic characteristics of the adult (Bruch, 1973a). She posited that, in individuals with severe eating disorders, "a basic disturbance can be identified in the way the sensation of hunger is experienced" (Bruch, 1973, p.44). The awareness of hunger was argued to be a developmental achievement arising from attuned interactions between the infant and mother. However, she claimed that "if the innate needs of the child and the environmental responses are poorly attuned to each other, a perplexing confusion in his conceptual awareness will result” (Bruch, 1973, p.51).

Whilst Bruch did not use the terminology of 'risk factors' as later outlined by authors such as Jacobi et al. (2004), her ideas have been reformulated in the light of modern understandings of aetiology (Treasure \& Cardi, 2017). Early caregiving experiences can be understood as distal risk factors for the development of AN within Bruch's model, rather than as a definitive cause. Bruch saw non-synchronous or mis-attuned early caregiving as giving rise to "deficiencies" that led to vulnerability to psychopathology at later developmental stages: "Lacking the essential tools for the subsequent developmental steps, a child is bound to be continuously bewildered and 
anxious, incapable of effective self-assertion in an incomprehensible world" (Bruch, 1973, p.63). Importantly for this thesis, Bruch incorporated ideas about reciprocal influence and circular causality into her model - ideas originally arising from cybernetics and general systems theory (Von Bertalanffy, 1972; Wiener, 1948), and key influences on the development of both family therapy and John Bowlby's attachment theory.

Indeed, Bruch's work includes many conceptual close cousins to central ideas explored in the attachment, mentalization and family therapy literature. Like Bowlby (1969), Bruch cited the work of Harlow on the effects of maternal deprivation in monkeys (e.g. Harlow \& Harlow, 1966), and she also cites an early study by Ainsworth \& Bell (1969) in support of her argument for the importance of early caregiving in influencing feeding trajectories. Although she did not develop her ideas around cognitive structures to the extent that Bowlby did, she came close to describing something akin to Bowlby's (1969) notion of internal working models, writing of how early experiences bias the infant's "perceptions and action patterns so that he responds to subsequent situations, at least initially, in the light of these early experiences, which in turn are in a continuous reciprocal or circular interaction with earlier structures and later transformations" (Bruch, 1973, p.63).

Bruch's foresight has been highlighted by Treasure \& Cardi (2017), who argue that her formulations regarding disturbed perceptions of emotions and bodily sensations anticipated the subsequent evidence base for concepts such as interoception and emotion processing in AN. Whilst Bruch saw early experiences as antecedents of the social and emotional difficulties characteristic of $\mathrm{AN}$, these were also seen as 
interacting with the physical impact of persistent malnutrition (Bruch, 1982). Evidence of larger deficits in theory of mind in individuals with acute AN, as compared with recovered individuals (Bora \& Köse, 2016), is one illustration of this interplay between physical and psychological functioning.

\subsubsection{Early roots of family therapy for eating disorders}

Bruch's ideas also find several close echoes in the concepts outlined by early pioneers of family therapy. For instance, Bruch's idea of excessive closeness between mother and daughter is conceptually similar to Minuchin's (1974) concept of enmeshment, which refers to an "extreme form of proximity and intensity in family interactions" (Minuchin, Rosman and Baker, 1978, p.30). For Minuchin, Rosman and Baker (1978), enmeshment was one of the four family interactional patterns that formed part of their concept of a psychosomatic family, in combination with the child's physiological vulnerability and their role in mediating family conflicts. For these authors, the development of AN was explained by the presence of these interactional patterns. The notion that excessive closeness in the family system plays a role in the development of AN is also fundamental to the ideas of Selvini-Palazzoli (1974), who used the term three-way matrimony to describe a family triangle in which the daughter with AN has to manage split loyalties to her parents through secret coalitions. Thus, ideas about family causation of mental health problems constitute a 'red thread' throughout the literature on psychotherapy for eating disorders in the post-war period from 1945 to approximately 1980.

The close links between psychoanalysis and the early family therapists have arguably been somewhat forgotten, perhaps because family therapists have tended to privilege discontinuities in their telling of the history of ideas (Flaskas, 2010). However, as 
pointed out by Byng-Hall (2003), many of the early family therapy pioneers were in fact psychoanalysts first - something which Byng-Hall credits with the speed and skill with which they were able to tune in to what was going on in families. This connection with psychoanalysis is particularly striking when we consider the key figures who were developing family therapy for eating disorders by the late 1970s and early 1980s, all of whom were psychoanalytically trained: Salvador Minuchin in Philadelphia; Mara Selvini Palazzoli in Milan; Helm Stierlin in Heidelberg; and finally Christopher Dare at the Maudsley Hospital in London.

In Minuchin, Rosman and Baker's (1978) seminal Psychosomatic Families, the authors presciently state that, whilst they have chosen to emphasise differences in their model from what has gone before, a reader in the future might well notice the continuity between their ideas and those of their predecessors. From today's standpoint, the early family therapists did indeed share many of the same assumptions as psychoanalysts such as Hilde Bruch, but their point of departure came in terms of therapeutic approach and site of intervention. Minuchin, Rosman and Baker (1978, p.90) make the point that:

"With the systems model it is unnecessary to posit historical causes and to tease them out during the therapeutic process. The past, or the significant parts of the past, are contained in the present. The early mother-child relationship is embodied in the current patterns of mutual regulation in the family. A change in that pattern - a transformation in the present - will change the meaning and the influence of the past." 
For Minuchin and his colleagues, family therapy did not require a rejection of psychodynamic or behavioural ideas, both of which were understood to have elements of truth and therapeutic value. Instead, the new emphasis was on treating the current context as a pragmatic means to effect change, since "the individual's responses will change if the context changes significantly, and so will his experiences" (Minuchin, Rosman and Baker, 1978, p.83). Following on from the formulation of the psychosomatic family (Minuchin et al., 1975), the structural approach to family therapy focused on changing the family interactional patterns that were postulated to be maintaining the child's AN symptoms: namely patterns of enmeshment, overprotectiveness, rigidity and lack of conflict resolution. These interactional patterns were to be disrupted through the use of an active therapist stance in which interactions would be blocked, encouraged or otherwise influenced by an array of interventions such as the use of enactment to encourage direct communication between family members.

In summary, the aim of the structural family therapy model was to change the structures that were seen to be maintaining the symptom - once altered, these changes were assumed to be preserved through the family's "self-perpetuating properties" (Minuchin, 1974, p.11).

\subsubsection{The Maudsley model}

Whilst the structural model of family therapy for eating disorders was undoubtedly hugely influential, by the mid-1980s it was receiving increasing levels of critique for its empirical and conceptual limitations. Early claims made for an $86 \%$ success rate, based on a sample of 53 cases in an observational study (Minuchin, Rosman and 
Baker, 1978), were criticised for weaknesses in methodology, such as the lack of independent assessors of outcome, whilst the evidence to support the psychosomatic family model itself was unconvincing (Kog \& Vandereycken, 1989). Also lacking was evidence for the superiority of family therapy to other approaches. Conceptually, the structural approach came under attack from feminist scholars for its lack of attention to power differentials both within the family unit and in wider society (Hare-Mustin, 1987; Hoffman, 1985). The adolescent patient with AN came to be a site for contested views about gender, family and society, with Kog \& Vandereycken (1989, p.21) suggesting that the patient had come to be seen as "no longer a piteous victim but a kind of misunderstood hero who acts as the secret family rebel, challenging patriarchal, collectivist, and self-sacrificial values and morals of traditional families... caught in the whirlpool of post-industrial civilization."

With a purely structural approach falling somewhat out of favour, the 1980s saw the rise to prominence of a model of family therapy developed at the Maudsley Hospital in London. This 'Maudsley model' drew from a variety of influences from different 'schools' of family therapy: Minuchin's (1974) structural model, strategic therapy (Haley, 1973), and the Milan group (Selvini Palazzoli, Boscolo, Cecchin and Prata, 1978) and its later off-shoots (the so-called 'Post-Milan' schools) (Boscolo, Cecchin, Hoffman and Penn, 1985). Psychodynamic influences were also present (Dare \& Eisler, 1997), and the approach can be characterised as integrative. The 1980s were a febrile time of transition for the family therapy field in general, in which the joke going around amongst trainees was: "blink and you'll miss a paradigm shift” (Flaskas, 2010). By integrating the newer 'post-modern' schools of family therapy, such as the PostMilan school, and by taking an 'agnostic' position with regards to the aetiology of AN 
- whilst also acknowledging its multifactorial origins (Dare \& Eisler, 1997) - the Maudsley team were able to counter the accusation of 'parent-blaming' whilst also positioning the treatment within the zeitgeist of systemic therapy. Moreover, the Maudsley group were able to combine both the glamour of the avant-garde ideas whirling through 1980s family therapy (Goldberg \& David, 1991; Stierlin, 1983), with the research data needed to gain a foothold in the academy. Crucially, the results of their randomised controlled trial (RCT) of family therapy (Russell et al., 1987) demonstrated superiority over individual therapy in reducing relapses following admission to hospital amongst adolescents with AN. Moreover, the research group's interest in expressed emotion (Vaughn \& Leff, 1976), discussed further in Chapter 2 (see also Simic, Jewell and Eisler, 2020), provided an intellectual and research focus that differentiated it from other schools and approaches to family therapy.

The 1987 trial was to be the first of nine RCTs completed to date for FT-AN, and this evidence base forms the basis for FT-AN's position as the first-line choice of treatment for adolescent AN (Couturier et al., 2020; National Institute for Health and Care Excellence, 2017). However, the model used in that trial and through the 1980s and 1990s was not manualised. In 2001, Lock et al. published the first treatment manual of the 'Maudsley model' or 'Maudsley method,' which was subsequently used in RCTs in the United States (e.g. Lock et al., 2005) and Australia (Le Grange et al., 2016) and came to be known as family-based treatment (FBT). In this thesis the term FBT will be used to refer to this manual and studies that utilised it, whereas FT-AN will be used as the umbrella term for eating-disorder focused family therapy (NICE, 2017; see also Chapter 2). 
The publication of the FBT manual paved the way for the further development of the evidence base for FT-AN, helping to establish its status as an Empirically Supported Treatment, and facilitating its wider dissemination. Importantly, it also served to define the treatment in terms of the content of the intervention (e.g. the use of a family meal in the second session) and led to a new articulation of its theoretical tenets. The authors stated that "mobilizing parents and family members as a resource is the most important theoretical position that sets this approach apart from other family and individual therapies for AN" (Lock et al., 2001, p.2). Perhaps even more decisively, several authors of the 2001 treatment manual wrote an Academy of Eating Disorders position paper on the role of the family in eating disorders (Le Grange et al., 2010): the authors stated that whilst family-based theories of causation of eating disorders, which they described as simplistic and erroneous, remain in circulation, modern family therapy approaches to adolescent eating disorders have reconceptualised the family from a site of pathology to a resource for recovery.

\subsubsection{Complex interventions revisited}

To summarise, the history of the development of FT-AN has some resemblance to the processes set out by the Medical Research Council framework (Craig et al., 2008). The development of Minuchin's family therapy model in Philadelphia, Dare and Eisler's Maudsley model in London, and Lock and Le Grange's FBT model in Chicago and Stanford all took place in the context of funded programs of research. Having said that, it should be noted that Minuchin's research outputs are not of the same order as those of the Maudsley or Chicago/Stanford groups, since they were not presented in peer-reviewed journal publications but in book form (Minuchin, Rosman and Baker, 1978); moreover, Minuchin did not pursue a research career, and by his 
own admission his interest in AN abated (see personal communication cited in Schmidt \& Asen, 2005). Nevertheless, the research he and his colleagues conducted in Philadelphia was to have an enormous influence on the field (Eisler, Wallis and Dodge, 2016). Their program of research explored the hypothesis that family interactions contributed to the development and maintenance of "psychosomatic conditions' (including AN), and that such interactions represented a viable and effective site for therapeutic interventions. In London, the Maudsley group researched an integrative family therapy approach that took an agnostic view on issues of causation (following Haley, 1973), but continued to research family processes, particularly through its line of research into expressed emotion (e.g. Eisler et al., 2000; Le Grange et al., 1992; Szmukler et al., 1985). In the past 20 years, the FBT manual (Lock et al., 2001), which positions families as a resource rather than a site of pathology, has helped to establish family therapy as an Empirically Supported Treatment through a number of RCTs (e.g. Le Grange et al., 2016; Lock et al., 2005, 2010).

In spite of these achievements, FT-AN arguably finds itself at a difficult point in its development. The field took a vital and necessary step in distancing itself from the "erroneous" theories that posited families as playing a role in the causation of AN (Le Grange et al., 2010), but this left it with a need to establish an alternative theoretical framework. Eisler (2005) reframed the interactional patterns that are sometimes seen in families with an adolescent with $\mathrm{AN}$ as the result of the family's response to illness (citing for example the work of Steinglass et al., 1987), rather than the cause of the eating disorder. This, along with the notion that families are a resource to be mobilized in the service of recovery, can be seen as representing two key theoretical tenets of the 
approach. There has also been attention paid, in terms of theory and research, to the constructs of parental self-efficacy (Byrne et al., 2015; Sadeh-Sharvit et al., 2018) and expressed emotion (Duclos et al., 2018; Eisler et al., 2007), as discussed further in Chapter 2. However, there has been a relative lack of specific, testable hypotheses about change mechanisms in FT-AN over the course of its development. Moreover, the evidence base such as it exists in relation to mechanisms of change, is largely made up of secondary analyses of RCTs that lack statistical power to address research questions about processes such as moderation and mediation (Hamadi and Holliday, 2020). Whilst exploratory analyses can play a role in hypothesis-generating, the reliance on such analyses to further scientific knowledge is also problematic in terms of research methodology, since researchers can have considerable degrees of freedom in terms of research questions and data analysis plans.

Thus, the problem for the FT-AN field has not been the lack of any theory, but a lack of testable theory of the kind that can inform improvements to treatment through an ongoing research process as per the MRC framework. To illustrate the point: in their important review of models of disordered eating, Pennesi \& Wade (2016) set as part of their inclusion criteria theoretical models that could be and had been tested: for instance models had to identify no more than six clearly conceptualised and measurable variables; furthermore, the relationship between at least two independent variables and the outcome variable, as delineated in the model, had to have been tested. No such models meeting inclusion criteria were identified for FT-AN. The authors comment on the lack of development of a testable theoretical underpinning for FTAN, which they suggest may disadvantage the further development of the treatment. 
To recap, the FT-AN field lacks theory in part due to the nature of its history: it has rejected the 'psychosomatic model' that provided some of its initial inspiration, but has not adequately established new theoretical principles through an iterative process of developing and testing theory through methodologically rigorous research. Nevertheless, in the big picture of the psychological therapy field, FT-AN's limitations could be characterized as the norm rather than the exception. Hofmann \& Hayes (2019) see the field of psychotherapy as currently emerging from several decades in which the research priority has been the development of specific treatment protocols for specific mental health syndromes. Whilst they posit that this historical period did many good things for the field, such as establishing the effectiveness of psychotherapy, the quest for 'protocols for syndromes' led to a narrowing of vision: as they put it, "theory suffered, and a more purely technological approach blossomed" (Hofmann and Hayes (2019), p.39). For Cuijpers et al., (2019, p.224), "it is as if we have been in the pilot phase of research for five decades without being able to dig deeper" into mechanisms of change in psychotherapy.

A key question for FT-AN is how to begin to take forward a research agenda that could begin to shed light on such mechanisms within the treatment. To do so will require both strong theory and constructs that can be measured in a reliable and valid way.

\subsubsection{The need for new concepts and theory in FT-AN}

FT-AN has yet to identify psychological constructs that can be said to be robust and well-replicated predictors of outcome. There are many factors that may account for this, including measurement error, lack of consistency in approaches to defining and measuring variables, and lack of statistical power. Indeed a lack of consistency in how 
outcomes are defined has been a major barrier to advancing the eating disorder field: for instance by hampering comparisons of treatments, and also preventing the development of a sound evidence base relating to predictors, moderators and mediators of outcome (Bardone-Cone et al., 2018; Vall \& Wade, 2015). The critically important issue of how to define eating disorder recovery is addressed via a qualitative study of social media users in Chapter 6 of this thesis.

However, the failure to build a well-replicated evidence base for psychological predictors of outcome in FT-AN may not only be due to research limitations such as a lack of standardized recovery definitions: might it also be due to failings in the predictor concepts themselves? The narrowing of focus over time described by Hofmann and Hayes (2019) in relation to the psychotherapy field in general can be said to apply to FT-AN. Despite the central role that parents play in the treatment, especially in early phases of therapy, research into parent and family variables does not appear to have been a high priority for the field (see Chapter 2 for description of the treatment and an overview of evidence into parent and family variables). One can speculate that one reason why research into family or parent variables has not been prioritized has been the legacy of the 'psychosomatic model' (Minuchin, Rosman and Baker, 1978), and the understandable desire on the part of model developers to distance the treatment from this work. However, paradoxically, FT-AN also needs family research in order to investigate its postulated mechanisms of change. But if relational concepts are to be included in the theory, research and practice of FT-AN, what might they be? And can they be sufficiently well operationalized and measured for the purpose of research? 


\subsubsection{Attachment and mentalization}

This thesis evaluates the relational constructs of attachment and mentalization in relation to treatment process and outcomes in FT-AN. Both concepts are multi-faceted and complex. Attachment can be understood as a broad, higher-order construct which has been operationalized in multiple ways, with a shared foundation in the conceptual and empirical work of Bowlby and Ainsworth (Bowlby, 1969; Ainsworth et al., 1978; see also Chapter 4). Attachment style, the construct investigated in the main study of this thesis, refers to a constellation of knowledge, expectations, and insecurities that people hold about themselves and their close relationships (Fraley \& Roisman, 2019). Mentalization refers to the capacity to understand the self and others in terms of intentional mental states such as feelings, desires, attitudes and goals (Luyten et al., 2020), whilst reflective function refers to this ability in the context of attachment relationships (Fonagy et al., 1991).

Bowlby (1969), a psychiatrist and psychoanalyst, who founded attachment theory in collaboration with Ainsworth (Ainsworth et al., 1978), described the theory as "an attempt to explain both attachment behaviour, with its episodic appearance and disappearance, and also the enduring attachments that children and other individuals make to particular others" (Bowlby, 1988, p.29). In order to explain these phenomena, Bowlby claimed that the key concept was the behavioural system, whose functioning was analogous to that of a physiological system keeping a measure such as temperature within an appropriate range (Bowlby, 1988). This conceptualisation was informed by Wiener's (1948) theory of cybernetics, which also influenced the development of family therapy. Bowlby postulated that the making and maintaining of affectional bonds was controlled by a "cybernetic system" within the central nervous system 
"utilizing working models of self and attachment figure in relationship with each other" (Bowlby, 1988, p.120).

The mentalization-based approach to psychopathology developed by Fonagy et al., (2002) posits that early attachment relationships play a key role in the developmental achievement of mentalizing ability. Marked mirroring of the child's emotional states by caregivers, for instance through the adult's facial expression, strongly influence the development of mentalizing and the infant's burgeoning sense of self. The emphasis on timely and contingent responses by the caregiver to the infant's cues represents a point of convergence with the ideas of Hilde Bruch (1973). Like Bruch, Fonagy et al.'s seminal Affect Regulation, Mentalization and the Development of the Self included both a close reading of the developmental research on infancy, and case examples of adults experiencing psychopathology. Whilst Fonagy et al. (2002) touched briefly on eating disorders in the book, the theory was first developed with borderline personality disorder in mind. As with attachment, mentalizing was conceived with the primary goal to inform the practice of psychotherapy; the development of Mentalization-Based Treatment (Bateman \& Fonagy, 1999) for borderline personality disorder took place in parallel to the development of the theoretical model. Bowlby, in his final book (A Secure Base), writes in the Preface with regret that whilst "attachment theory was formulated by a clinician for use in the diagnosis and treatment of emotionally disturbed patients and families, its usage hitherto has been mainly to promote research in developmental psychology" (Bowlby, 1988, p.ix). However, over the past 30 years there has been an explosion in interest in the clinical implications of attachment, with attachment-based therapeutic models being developed for individuals, couples and families across the life-course. 


\subsubsection{Attachment, mentalization and eating disorders}

Whilst attachment and mentalization represent relatively new concepts within the theory of FT-AN, both have been widely researched in the context of the wider eating disorder field. Individuals with eating disorders experience higher rates of insecure attachment and mentalizing difficulties than community controls (Caglar-Nazali et al., 2014; Tasca \& Balfour, 2014; see also overview in Chapter 3). There have been efforts to integrate attachment and mentalizing ideas into treatment models, such as the development of mentalization-based treatment for eating disorders (Robinson et al., 2018). In recent years, researchers have also begun to investigate the role of attachment and mentalizing as predictors of alliance and outcome in eating disorder treatment. For instance, therapeutic alliance was found to be predicted by attachment (Folke et al., 2016) and mentalization (Katznelson et al., 2019) in a Danish sample receiving treatment for bulimia nervosa. Treatment outcomes have also been predicted by both attachment (Illing et al., 2010a) and mentalization (Kuipers et al., 2018; Rothschild-Yakar et al., 2010; Schulte-Rüther et al., 2012).

\subsubsection{Attachment, mentalization and family therapy}

Attachment was initially formulated as a theory at around the same time as the first wave of family therapists emerged in the 1960s and 1970s. John Byng-Hall, whose seminal Rewriting Family Scripts provided an influential synthesis of attachment and systemic ideas, comments that the family therapy field was surprisingly slow to integrate attachment into family therapy (Byng-Hall, 1995). However, as Bowlby's comments cited earlier illustrate, uptake of attachment ideas within the psychotherapy field in general appears to have taken until the 1990s. In the family therapy field, notable empirically supported treatment models that have used attachment as a 
theoretical basis include Diamond et al.'s (2002) Attachment-Based Family Therapy model for adolescent depression and self-harm, and Emotionally Focused Couple Therapy (Johnson, 2019). Meanwhile Dallos (2004, 2006) has also integrated attachment, systemic and narrative therapy ideas, and illustrated them with reference to eating disorders (e.g. Crittenden \& Dallos, 2009; Dallos \& Denford, 2008).

Finally, Wallis et al. (2017a) made an important theoretical contribution to the FT-AN literature with their qualitative study of 16 adolescents and 28 parents who had completed FBT following inpatient admission within the context of a clinical trial. All adolescent participants had achieved positive clinical outcomes at 12-month followup. Using grounded theory to analyse the participants' interviews, the researchers developed a conceptual model of family relationships before, during and after treatment. Participants described a process whereby family members experienced stress, conflict and isolation prior to treatment, but during FBT the structure of treatment, including regular appointments, initial intensity and consistent support created a sense of relational containment. For parents, the support of the therapeutic team provided them with a sense of increased confidence and security. Attachmentrelated themes were also present in the adolescents' responses, describing an increased sense of trust and connection with parents. Following FBT, families reported being closer, with adolescents seeming more confident and emotionally expressive. Whilst the study has the limitation of selection bias in terms of its small sample of participants, all of whom had positive treatment outcomes, the study nonetheless is one of the first to take a longitudinal view of family processes over the course of treatment, and to include the wider treatment system of family and team. 


\subsection{Summary and outline of this thesis}

FT-AN is a field in which there is a need for theory and research that can inform the further development of the intervention, in line with the principles of the Medical Research Council framework for complex interventions (Craig et al., 2008). The primary aim of this thesis is to investigate the constructs of attachment and mentalization as predictors of outcome in FT-AN. In order to do so, the main study of the thesis (Chapter 5) is a prospective observational study in which a specific theoretical model will be tested in which attachment and mentalization predict categorical outcome via the mediating variable of therapeutic alliance. In so doing, the study will speak to a key issue in psychotherapy research: whether change happens through specific or common factors (Cuijpers et al., 2019). Attachment and mentalization represent relational constructs that may have roles to play in FT-AN that are specific to the nature of the treatment and the disorder, as outlined further in subsequent chapters of the thesis. At the same time, FT-AN may work through common factors such as the mobilization of hope and expectancy for change. By investigating the potential mediating role of alliance, the study seeks to shed further light on how common and specific factors might operate within FT-AN.

Secondary aims of the thesis are to synthesize the evidence base for FT-AN through a narrative review; to systematically review the evidence pertaining to attachment and mentalization in relation to child and adolescent eating pathology; to conduct a systematic review of measurement properties of attachment measures in middle childhood and adolescence; and to undertake a qualitative analysis of how eating disorder recovery might be defined from the perspective of users of eating disorder recovery-related social media forums. 
The structure of the thesis is as follows:

Chapter 2 will present a narrative review of the evidence base for family therapy for child and adolescent eating disorders, including evidence relating to efficacy, prediction of outcome, and treatment processes such as alliance. The chapter will consider the evidence base from a common factors perspective (Sprenkle et al., 2009), and will apply the notion of epistemic trust (Fonagy et al., 2015) to FT-AN. Epistemic trust refers to the individual's willingness to consider new knowledge from another person as trustworthy, generalizable, and relevant to the self. It is argued that mechanisms of change in FT-AN might include the fostering of hope and epistemic trust in the context of family therapy that is situated within an expert multidisciplinary team. In addition, theoretical ideas are advanced in relation to the potential relevance of attachment and mentalization to outcome in FT-AN.

Chapter 3 reports the first systematic review of the evidence for an association between attachment, mentalization and eating pathology in children and adolescents. The review identifies large gaps in the evidence base as regards to attachment and mentalization within child and adolescent clinical populations with eating disorders. Moreover, this chapter addresses the issue of the possible aetiological role that attachment and mentalization might play within eating disorders.

Chapter 4 addresses the key issue of measurement of attachment. If attachment is to play a role in FT-AN theory and research, we need to be confident in our ability to measure it in a valid and reliable way. Unfortunately, however, previous reviews (e.g. 
National Institute for Health and Care Excellence, 2015) have identified poor methodology and sub-optimal psychometric properties for measures of attachment in middle childhood and adolescence. The chapter presents the first systematic review of attachment measures in middle childhood and adolescence to include all available measures, whether they be self-report, interview or task-based assessments. Individual studies were assessed for risk of bias as well as for the adequacy of psychometric properties, using the COnsensus-based Standards for the selection of health Measurement INstruments (COSMIN) (de Vet et al., 2011).

Chapter 5 presents the main study of the thesis, which is a prospective observational study of 192 families undertaking FT-AN across three clinical sites in the United Kingdom. The study tests a specific model in which attachment and mentalization predict outcome, with therapeutic alliance serving as a mediator.

Chapter 6 reports a qualitative study of how users of social media forums define eating disorder recovery. Using thematic analysis of comments posted on eating disorder recovery forums on the Reddit website, the study highlights the importance of positive aspects of well-being and quality of life, including social connection. The presence of attachment themes in the comments will also be highlighted.

Finally, Chapter 7 will provide a discussion and synthesis of the overall body of research presented in this thesis. 
All chapters are either presented as stand-alone journal articles, reproduced exactly as published or accepted, or adapted from manuscripts that are either published or under review (as indicated at the start of each chapter). 
Chapter 2 - Family therapy for child and adolescent eating disorders: A critical review

This chapter is presented as a published journal article. Other than the updated literature review at the end, it is an exact copy of the following publication:

Jewell, T., Blessitt, E., Stewart, C., Simic, M. and Eisler, I. (2016). Family Therapy for Child and Adolescent Eating Disorders: A Critical Review. Family Process, 55, $3,577-594$ 


\subsection{Abstract}

Eating disorder-focused family therapy has emerged as the strongest evidence based treatment for adolescent anorexia nervosa supported by evidence from nine RCTs, and there is increasing evidence of its efficacy in treating adolescent bulimia nervosa (three RCTs). There is also emerging evidence for the efficacy of multi-family therapy formats of this treatment, with a recent RCT demonstrating the benefits of this approach in the treatment of adolescent anorexia nervosa. In this article we critically review the evidence for eating disorder-focused family therapy through the lens of a moderate common factors paradigm. From this perspective, this treatment is likely to be effective since it provides a supportive and non-blaming context that one, creates a safe, predictable environment that helps to contain anxiety generated by the eating disorder, two, promotes specific change early on in treatment in eating disorder related behaviors, and three provides a vehicle for the mobilization of common factors such as hope and expectancy reinforced by the eating disorder expertise of the multidisciplinary team. In order to improve outcomes for young people, there is a need to develop an improved understanding of the moderators and mediators involved in this treatment approach. Such an understanding could lead to the refining of the therapy, and inform adaptations for those families who do not currently benefit from treatment. 


\subsection{Introduction}

In this article we provide a critical review of the evidence for eating-disorder focused family therapy (Eisler, Le Grange and Lock, 2015) for children and adolescents. We will look at the evidence as it pertains to anorexia nervosa (AN) and bulimia nervosa $(\mathrm{BN})$, the two disorders that have been most-studied in the child and adolescent population. AN is a disorder characterized by significantly low weight, dietary restriction, intense fear of weight gain and distorted body image, and consists of restricting and binge/purge subtypes (APA, 2013). BN is characterized by a similar fear of weight gain, as well as binge eating followed by compensatory behaviors such as vomiting or laxative abuse. Using DSM-5 criteria (APA, 2013), the lifetime prevalence of $\mathrm{AN}$ is $1.7 \%$, whilst for $\mathrm{BN}$ it is $0.8 \%$ (Smink et al., 2014).

The evidence for treatment of child and adolescent eating disorders has been reviewed extensively in recent years. Systematic reviews have concluded that family therapy for adolescent anorexia nervosa (FT-AN) ${ }^{1}$ has strong evidence of efficacy (Watson and Bulik, 2013; Lock, 2015), with higher rates of recovery at 6 and 12-month follow-up as compared to individual therapy (Watson and Bulik, 2013; Couturier et al., 2013a; Downs and Blow, 2013; Lock, 2015). FT-AN is the recommended treatment for adolescent AN in clinical guidelines for a number of countries, such as the U.S (APA, 2006) and UK (NICE, 2004), and is the only well-established treatment available for this population (Lock, 2015). The evidence for psychosocial treatments of $\mathrm{BN}$ is more limited, but family therapy for adolescent bulimia nervosa (FT-BN) has been found to be superior to cognitive behavior therapy (CBT) in a recent RCT (Le Grange et al., 2015). Previous studies of FT-BN have shown it to achieve comparable outcomes to CBT (Schmidt et al., 2007) and superior outcomes to supportive psychotherapy (Le 
Grange et al., 2007). Evidence is also accumulating for the efficacy of multi-family therapy formats, in which several families with a child with an eating disorder come together for intensive group treatment (Eisler et al., 2016b; Simic and Eisler, 2015). In this article we will review the evidence for eating disorder focused family therapy through the lens of the common factors paradigm (Sprenkle, Davis and Lebow, 2009; Wampold, 2010). This perspective emphasizes the importance of variables which apply across all therapeutic models, such as client and therapist factors, therapeutic alliance, therapist allegiance to the treatment model, and the mobilization of client hope or expectancy. Since these common factors have been argued to account for a much greater proportion of variance in outcome than the specific model employed in treatment (Asay and Lambert, 1999), the common factors paradigm lends itself to a critical appraisal of the evidence for any empirically supported treatment - particularly claims of greater efficacy relative to other treatments. Whilst the common factors paradigm has sometimes led to polarized debates about the relative merits of common factors as compared with specific models and techniques, it is possible to take a 'moderate common factors' position: that is, both accepting that common factors are key ingredients to successful psychotherapy, and yet remaining open to the possibility that specific aspects of a treatment model or particular techniques can be superior for particular difficulties or sub-groups of clients (Sprenkle, Davis and Lebow, 2009). In this article we will be adopting just such a position in our review of the evidence. 


\subsection{Description of the treatment}

\subsubsection{Family therapy for Anorexia Nervosa (FT-AN)}

The core features of FT-AN include the following: a clear focus on working with the family to help their child recover, coupled with a strong message that the family is not seen as the cause of the problem; expecting the parents to take a lead in managing their child's eating in the early stages of treatment; externalizing the eating disorder; and a shifting of focus on to adolescent and family developmental life cycle issues in the later stages of treatment (Eisler, Wallis and Dodge, 2015). Whilst the treatment has been manualized by a number of research teams, the different treatment manuals all adhere to the core principles just outlined (for more detailed discussion see Eisler, Wallis and Dodge, 2015). In this article we describe the treatment as operationalized in the Maudsley service manual (Eisler et al., 2016a).

In our conceptualization the treatment is an integrative four-phase systemic treatment model delivered by a therapist in the context of a specialist multi-disciplinary eating disorders team setting. In Phase One, Engagement and development of therapeutic alliance, the child is assessed alongside their parent/s. The assessment process has a strong multidisciplinary focus that includes a psychiatric frame (confirming the diagnosis, identifying co-existing problems such as anxiety, depression or self-harm), a medical/pediatric frame (evaluating medical and physical risk that have to be managed safely), an individual psychological frame (exploring motivation to change, identifying cognitive and temperamental characteristics of the young person) as well as a family systems frame (developing a systemic formulation and identifying areas of family strengths and resilience) but the assessment is also the beginning of the process of engagement between therapist and family. The therapeutic engagement 
should include all family members including the young person even though they may often appear to be a reluctant participant at first. The therapist offers information about AN and the physiological as well as psychological effects of starvation (Keys et al., 1950) highlighting the fact that many of the phenomena associated with eating disorders are characteristic of anyone in a state of starvation. The providing of expert information thus becomes part of the process of externalizing the illness reinforced by “externalizing conversations" (White and Epston, 1990), with the aim of developing a therapeutic ethos of non-blame and guilt reduction. The inclusion of a medical examination at assessment helps to engender an appropriate atmosphere of concern, promotes a message to the parent/s that they need to act urgently to reverse the effects of starvation, and contributes to the development of a safe base for treatment. During the final part of the assessment, a meal plan is presented to the parents as a 'prescription' for recovery. The family are reassured that the therapist will work alongside them, beginning with a family meal within the next week where further advice and information will be provided by the therapist. Weekly appointments are the norm during the early stages of treatment whilst parent/s and patient struggle with the demands of feeding and weight restoration.

In Phase Two, Helping families manage the eating disorder, the therapist continues to encourage parents to take a lead in managing their child's eating, while emphasizing the temporary nature of this role. The child is weighed by the therapist at the start of each session with the focus of therapy then being dictated by the weight trajectory. Therapeutic tasks in this phase will include detailed exploration of what happens at mealtimes, exploring parental roles, increasing parents' sense of agency, for instance through challenging beliefs about the impossibility of parental action, and sharing 
examples of what other families have done to overcome similar difficulties. While much of the focus is on how the parents can help their child it is important for the therapist to maintain a good engagement with the young person, discussing broader goals that can be achieved as physical health is gradually restored.

With most families this phase tends to have a fairly behavioral focus and relational issues that are raised are noted as important but left to be "addressed at a later stage when the physical concerns have been resolved". With a minority of families progress at this stage is, however, more problematic and broader issues need to be addressed with the family. Most commonly this slower progress happens in the context of a more complex individual and/or family presentation (Simic et al., 2016). This will tend to include higher levels of comorbidity, the young person may be emotionally dysregulated and the clinical picture may include episodes of self-harm and a binge/purge rather than a pure restricting presentation. At a relational level there is often increased negativity or hostility and insecure patterns of attachment (Jewell et al., 2016b). Attachment and emotion focused family interventions (Diamond et al., 2014; Robinson et al., 2015) may be usefully employed to overcome the impasse that can develop at this stage.

Therapy moves to the third stage, Exploring issues of individual and family development, once weight restoration has largely been achieved or is well under way. This marks a move in therapy towards handing back age-appropriate responsibility to the patient and supporting parent/s to re-focus on their individual needs and those of other family members. The presence of an eating disorder, like any other serious and life-threatening condition, can cause great disruption to family organization (Eisler, 
2005). These effects are explored and addressed during this phase of treatment. The move to Phase Three is generally also marked by a change in the nature of the therapeutic alliance. In the early stages of treatment the therapeutic alliance tends to be characterized by dependence on the therapist, reinforced by the therapists' expertise, willingness to give advice and share experiences of successful strategies that other families have employed. The family's dependency on the therapist in the early stage of treatment parallels the temporary increased dependency of the young person on the parents that the therapy advocates. Just as at the later stage of therapy the parents are encouraged to start handing back responsibility to the young person (and the young person is encouraged to reassert their wish for independence), the therapy also needs to address the dependent relationship of the family on the therapist.

Ending treatment, discussion of future plans and discharge is the final phase of treatment. The therapist in Phase Four may encounter parental anxiety which is out of step with their child's progress and therefore sessions in this phase include discussions about relapse prevention, tolerance of uncertainty, reviewing the course of recovery and some reflection on the expertise of parents and child to manage future difficulties.

\subsubsection{Multi-Family Therapy for Anorexia Nervosa (MFT-AN)}

MFT-AN draws on the four-phase treatment model in a group therapy for 5-7 seven families at one time. MFT-AN commences with an introductory afternoon comprising a lecture highlighting the psychological and physical consequences of starvation. Parents and young people then meet a 'graduate family' - a family who have previously been through the MFT-AN process - who share their experiences of the group. This meeting is followed by four intensive days of therapy with up to five 
follow-up days over the following six to nine months. The intensive nature of the treatment has been described as creating a 'hothouse effect' (Asen and Scholz, 2010) which makes it a powerful context for mutual learning, reducing the sense of isolation and stigma and increasing a sense of hope and the likelihood of change. In addition to the program of therapeutic sessions (see Simic and Eisler, 2015, for details), families also have their meals and snacks together in communal areas, providing multiple opportunities for in-vivo learning and support.

\subsubsection{Family therapy for bulimia nervosa (FT-BN)}

FT-BN differs somewhat from the FT-AN model. FT-BN sessions are far more likely to feature separated sessions, with the therapist meeting the young person and parent/s on their own at least early on in treatment. Greater attention is focused on building a therapeutic engagement between therapist and the young person in order to ensure that issues of motivation to change and building trust within the family can be addressed early on. Early sessions with parents provide psychoeducation, practical parenting skills and coaching with an emphasis on reducing criticism, blame and guilt. Validation skills are promoted as a way of supporting future change. Early separated interventions in FT-BN provide a foundation for later conjoint sessions, when issues of communication and collaboration can be enhanced to support behavioral change.

\subsubsection{Multi-family therapy for bulimia nervosa (MFT-BN)}

MFT-BN shares some similarities with MFT-AN, with similar benefits arising from the group process as described above. It provides a group learning opportunity but with sessions spaced weekly over four months, allowing for a slower process of change, and providing space for reflection and practice of the skills learned. Similarly to FT-BN, initial group meetings have more separate parallel sessions with young 
people and the parents. The shared context of the MFT group allows parents to feel that they are not alone in dealing with the frustrations and difficult behaviors of their child. Alongside systemic tasks and exercises, elements of both Dialectical and Cognitive-Behavioral approaches are combined to address the unique needs of this patient group (Stewart et al., 2015).

\subsection{Efficacy research}

The eating disorders field provides a challenging context in which to conduct research. The relative rarity of AN means that multiple sites are often needed to recruit sufficient numbers into an RCT (Watson and Bulik, 2013). In the case of BN, recruitment to studies can also be problematic, since help-seeking is typically delayed by $4-5$ years (Turnbull et al., 1996), meaning that many adolescents with $\mathrm{BN}$ are not presenting to services until adulthood. A further challenge for research is that the urgent medical risks presented by eating disorders, particularly $\mathrm{AN}$, mean that providing a wait-list condition in any efficacy trial raises important ethical issues (Watson and Bulik, 2013). This means that treatments under investigation have to go up against other credible, bona fide treatments. This provides a sterner test of efficacy, but provides a methodological challenge in a field with few established treatments.

\subsubsection{Relative efficacy of FT-AN}

Three RCTs have investigated the efficacy of family therapy as compared with individual therapy for AN. The first of these, by Russell et al. (1987), was conducted at the Maudsley Hospital in London, and involved a sample of 57 participants with AN and 23 participants with BN. Participants included both adolescents and adults, and they were divided into four sub-groups: one group with $\mathrm{BN}$, and three groups with 
AN grouped by duration of illness and age at onset of illness. These subgroups were then randomized to family or individual therapy. After one year of treatment, in the subgroup of AN participants aged under 19 at illness onset and an illness duration of less than three years $(n=21) 90 \%$ of those receiving family therapy achieved better categorical outcomes (based on weight, menstruation status and presence of bulimic behaviors) compared to $18 \%$ of those receiving individual therapy. These differences persisted at five-year follow-up (Eisler et al., 1997). The findings of this influential study are compromised by a number of limitations. Firstly, the lack of manualization of the treatments delivered means that the study would no longer meet criteria for inclusion as evidence for an empirically supported treatment (Lock, 2015). Secondly, the sample size for the group which showed superior efficacy of FT-AN is very small. Thirdly, the supportive individual therapy arm was not a bona fide therapy, since it lacked a theoretical model of change or clear focus for treatment.

Robin et al., (1999) conducted a small RCT ( $\mathrm{n}=37$ ) comparing family therapy and individual therapy in the treatment of adolescents (aged 11-20) with AN. The behavioral family systems therapy (BFST) used by Robin and colleagues had many similarities with the approach developed at the Maudsley (Robin et al., 1999; Eisler, Wallis and Dodge, 2015). The comparison treatment consisted of Ego-Oriented Individual Therapy (EOIT), a treatment derived from psychodynamic principles. The therapist saw adolescents on a weekly basis, and met with parents fortnightly, although unlike in the family intervention the parents were encouraged not to get directly involved in the management of mealtimes. BFST resulted in significantly greater increases in body mass index (BMI) at end of treatment (BFST mean change 4.7; EOIT mean change 2.3) and at one-year follow-up (BFST 5.5; EOIT 3.2). Approximately 
two thirds of the adolescents reached the target weights (set individually by their pediatrician) at end of treatment with no differences between the treatment arms. Significantly more girls in the BFST group (94\%) than in EOIT (64.4\%) had resumed menstruation by the end of treatment.

The largest efficacy trial comparing FT-AN and individual work was conducted by Lock et al. (2010). 121 adolescents with AN were randomized either to a manualized family therapy based on the Maudsley approach, referred to as Family Based Treatment (FBT), or to an individual therapy referred to as Adolescent Focused Therapy (AFT) a modification of EOIT used in Robin et al.'s (1999) study. At the end of treatment there was no statistical difference between the two treatments in terms of rates of full remission, although FBT was statistically superior in terms of partial remission, participants' BMI percentile, and hospitalization rates. FBT was significantly superior in terms of rates of full remission at six-month (FBT $=40 \%$ vs. AFT $=18 \%)$ and twelve-month follow-up $(\mathrm{FBT}=49 \%$; AFT $=23 \%)$. Full remission was defined as a participant achieving a minimum of $95 \%$ expected body weight adjusted for sex, age and height, and scores within one standard deviation of community norms for self-reported eating pathology.

\subsubsection{Relative efficacy of FT-BN}

There have been three RCTs of FT-BN. Le Grange et al., (2007) compared family therapy (using a modification of their FBT manual) with supportive psychotherapy in a sample of 80 adolescents. FBT-BN emerged as significantly superior at end-oftreatment (39\% binge-and-purge abstinence vs. $18 \%$ in the supportive therapy arm,). At six-month follow-up, abstinence rates had reduced in both groups (29\% for FBT- 
BN vs. $10 \%$ for supportive therapy), but FBT-BN retained its superiority over supportive therapy. However, the assessment of treatment response was not blind to treatment condition, thus posing a risk of bias. A further test of the efficacy of FT-BN was provided by Schmidt et al., (2007), in a comparison with guided self-care CBT $(n=85)$. In this study there were no significant differences between groups on the primary outcome, abstinence from bingeing and purging, either at six months (end of treatment) (FT-BN 13\%; CBT 19\%), or at follow up at twelve months when abstinence rates stood at $41 \%$ for the FT-BN group, and $36 \%$ for the CBT group. However, the CBT group had an earlier reduction in binge frequency. Schmidt et al., (2007) highlight a low rate of recruitment because of some of the older adolescents' unwillingness to involve their parents in the treatment, but adolescents who received FT-BN continued to make more improvements after the end of treatment than those seen on their own. Treatment costs were significantly lower for the CBT arm.

In the most recent RCT, Le Grange et al., (2015) compared FBT-BN, CBT and supportive psychotherapy in a sample of 130 adolescents. Recruitment rates were structured in such a way that more adolescents were randomized to FBT-BN ( $\mathrm{n}=51)$ and CBT $(n=58)$ than the supportive psychotherapy arm $(n=20)$. Compared to CBT, abstinence rates were higher for FBT-BN at end of treatment (39\% for FBT-BN vs. $20 \%$ for CBT) and at six-month follow-up (44\% for FBT-BN vs. $25 \%$ for CBT). At one-year follow-up there were no differences between groups. Rates of hospitalization were significantly lower for FBT-BN. The risk of bias is reduced in this study as compared with the earlier Le Grange et al. (2007) study, due to the use of independent assessors. 


\subsubsection{Efficacy trials comparing different forms of FT-AN}

Four studies have compared the efficacy of different forms of FT-AN. Le Grange et al. (1992) conducted a pilot RCT comparing conjoint FT-AN, in which family members were seen together for therapy, with separated FT-AN, in which adolescents and parents were seen separately by the same therapist. This small study $(\mathrm{n}=18)$ found no significant differences between the two forms of treatment. Eisler et al. (2000, 2007) conducted a larger RCT $(n=40)$ comparing separated and conjoint FT-AN, replicating the finding that overall neither was superior, either at end of treatment or at 5-year follow-up. The lack of difference between the two treatment arms was important because it challenged the prevailing theoretical assumptions of the model, since the findings further undermined the idea that family members needed to be seen together, in order to intervene in family patterns that might be illness-maintaining. Moreover, whilst at aggregate level there was no difference between treatment arms, families rated as high in maternal criticism - an aspect of the measure expressed emotion (Leff and Vaughn, 1985) - achieved significantly better outcomes when offered separated FT-AN, a finding that was sustained at 5-year follow-up.

A recent RCT by Le Grange et al. (2016) (n=107) compared conjoint FBT with a manualized form of separated FBT, which they name Parent-Focused Treatment (PFT). In PFT, a nurse weighs the adolescent, assesses medical stability, and provides brief supportive counselling, with the total individual contact time limited to 15 minutes. The adolescent's weight and any other pertinent information is then communicated to the therapist, who then sees the parents for 50 minutes with a similar treatment focus to that used with the whole family in FBT. Remission, defined as in the Lock et al. (2010) study, was higher in PFT than in conjoint FBT at end-of- 
treatment (six months) (43\% vs. 22\%), but did not differ statistically at 6- or 12-month follow-up. Lower parental expressed emotion predicted higher rates of remission in both study arms, but in contrast to the Eisler et al. studies $(2000 ; 2007)$ treatment response in families with high expressed emotion did not differ according to treatment. Whilst the study further undermines the idea that conjoint sessions are a necessary ingredient of successful treatment, the findings also demonstrate the benefits of conjoint treatment for certain patient groups. For instance, patients with higher eating disorder-related obsessionality benefitted more from FT-AN than PFT, in keeping with previous findings suggesting that conjoint treatment is more beneficial for this group of patients as compared to separated treatment (Eisler et al., 2000) or individual work (Lock et al., 2010). Finally, Lock et al. (2005) have investigated dose of treatment, comparing outcomes of short (10 sessions over 6 months) vs. long forms (20 sessions over 12 months) of FBT In this study of outpatient treatment $(n=86)$, there were no significant differences in outcomes between the two treatment arms. The study suggests that there are a number of treatment 'responders' for whom FBT works well within a short duration of time. For this group, increased contact hours appear to confer no additional benefit. However, non-intact families, and families where the young person had high levels of eating disorder-related obsessionality, benefitted significantly more from the longer form of treatment.

\subsubsection{Efficacy of FT-AN compared with generic family therapy approaches}

From a moderate common factors perspective, an obvious question to ask is whether the efficacy of FT-AN is due to specific ingredients of the model, or whether an alternative family therapy model might achieve equivalent outcomes. Thus far only one RCT, conducted by Agras et al. (2014), has been designed to help answer this 
question. This study of outpatient treatment $(\mathrm{n}=164)$ compared two forms of manualized family therapy: FBT and Systemic Family Therapy (SyFT - Pote et al., 2001). The latter was a 'generic' form of family therapy, not specifically designed for treating adolescent AN. At end of treatment, there were no significant differences between treatments in terms of the primary outcome measures of percentage of ideal body weight and remission. However, participants receiving FBT gained weight faster early on in treatment, spent fewer days in hospital, and treatment costs were lower, suggesting overall advantages of FBT. The study therefore provides support for the view that therapists adhering to a FT-AN treatment manual will achieve superior results overall as compared to those utilizing a more generic approach.

Interpreting the findings of this study is complicated by two potential confounding variables: the eating disorder expertise of clinicians, and the role of specialist service contexts. Participants in both arms of the Agras et al. study were seen in specialist eating disorder services by therapists with an average of 6 years of experience of working with eating disorders. SyFT did not preclude a focus on the eating disorder, and given the treatment context and the therapists' expertise, it is understandable that many families brought the discussion of the child's eating disorder to therapy as a treatment priority. As a result, the two forms of family therapy investigated may have been more similar than it would otherwise seem (Blessittt, Voulgari and Eisler, 2015). These issues are illustrated by Godart et al.'s (2012) RCT conducted in France ( $\mathrm{n}=60)$, which investigated whether family therapy improved outcomes in adolescents treated in hospital. The family therapy model used in the study was not FT-AN, but a more generic approach in which family dynamics were conceptualized as being involved in the development and maintenance of the eating disorder. Adolescents receiving family 
therapy achieved significantly better outcomes compared with those receiving treatment as usual. Consequently, we can surmise that family therapy can have a beneficial impact on outcome even if the FT-AN model is not used. However, given that again the study was conducted by therapists with significant eating disorders expertise in a specialist service, it does not follow that family therapists without eating disorders expertise, working in non-specialist services, can achieve equivalent results. We discuss the impact of service context in more detail later in this article.

\subsubsection{Efficacy of MFT-AN and MFT-BN}

Currently one RCT has been conducted examining the efficacy of MFT-AN (Eisler at al., submitted) demonstrating significantly improved categorical outcomes for families who attended MFT-AN in addition to single family FT-AN. The potential benefit of MFT-AN is also indicated by several smaller studies. For instance, Salaminiou et al. (2015) report good or intermediate outcomes achieved by 6 months in $62 \%$ of the 30 families receiving MFT-AN. Gabel et al. (2014) in a case matched comparison report higher weight gain in adolescents receiving MFT-AN as compared to treatment as usual. Finally, Marzola et al. (2015) reports a brief treatment adaptation of MFT-AN, in which treatment was delivered over five full consecutive days. A follow-up of between 2-5 years of 74 patients showed that nearly $90 \%$ had achieved full or partial remission.

Research findings on the efficacy of MFT-BN are currently scarce. Stewart et al. (2015) have described the development of a MFT-BN group delivered in an outpatient context over 20 weeks in 1.5 hour long sessions. Preliminary findings reported in their paper $(n=10)$ suggest that the group reduces eating pathology and depression, and 
increases adaptive coping skills. Thus MFT-BN currently shows promise, but further research is needed with larger samples and comparison groups.

\subsection{Findings on implementation and service context}

\subsubsection{Implementation Studies}

A small number of studies have looked at the implementation of FT-AN (see Couturier and Kimber (2015) for a recent review). Three small dissemination studies investigated whether clinicians who were unfamiliar with FT-AN could be trained in the approach over two days, following which their clinical outcomes were evaluated. In all three studies, clinical outcomes improved (Couturier et al., 2010; Tukiewicz et al., 2010; Loeb et al., 2007). Two larger retrospective studies conducted by Wallis et al. (2007) and Hughes et al. (2014) provide evidence of substantial reductions in rates of hospitalization, readmission and the length of hospital admissions following the adoption of FT-AN by children's hospitals in Sydney and Melbourne.

2.5.2 Service context as a possible common factor in the treatment of eating disorders One of the main limitations of all the studies discussed above is that they do not take into account the potential impact of therapist eating disorders expertise and the role of specialist service context, which can be seen as common factors across all the treatment studies, conducted thus far in the adolescent eating disorder field.

The findings of a naturalistic study by House et al., (2012) sheds some light on these issues. The study compared all adolescents with an eating disorder in London over a two-year period whose treatment followed different referral and treatment pathways that were determined by local commissioning arrangements that either allowed direct 
referral from primary care physicians to a specialist outpatient eating disorders service or followed a stepped care model with initial referrals going to the local generic child and adolescent mental health services (CAMHS). There were considerable differences between those who had access to the specialist care pathway compared to those whose initial referral was to generic CAMHS teams. The specialist pathway had 2-3 times higher case identification rates, two and a half times lower rates of hospital admissions during the first 12 months following referral and considerably greater consistency of care with one treatment provider. While this does not provide direct evidence for the effectiveness of the FT-AN (although this was the main treatment mode in the specialist services), it suggests that other factors are operating, such as referrers' expectation, clinician confidence, the availability of a specialist eating disorders multidisciplinary team that is able to manage complex cases from the start, and the mobilization of expectancy effects (Eisler, Wallis and Dodge, 2015) which RCTs do not account for but which appear to have a major impact on outcome.

A related finding comes from a study by Murray, Griffiths and Le Grange (2014). This small study $(n=29)$ found that collegiate alliance - the perceived alliance between case-involved professionals - predicted drop-out from FBT and was negatively correlated with eating pathology at end-of-treatment. One possible explanation for this could be that support from a likeminded multidisciplinary team may be important in FT-AN due to the emotional challenges that clinicians can experience in using this approach. Couturier et al. (2013b) conducted interviews with FBT therapists, from which it emerged that clinicians can feel anxious about certain therapeutic tasks, such as weighing the patient and completing family meals. Kosmerly et al. (2015) found that greater clinician anxiety was associated with therapists being less likely to weigh 
the client at the beginning of a session. Similarly, Robinson and Kosmerly (2015) found just under a third of FBT therapists in their study reported that clinicians' own emotions negatively influenced treatment decisions. Thus one of the things which specialist teams may provide is an environment in which therapists can receive supervision and support, thereby strengthening treatment fidelity (Couturier and Kimber, 2015), but also attending to the emotional challenges which may lead to poorer clinical outcomes.

\subsection{Predictors, moderators and mediators of outcome}

\subsubsection{Predictors of outcome}

Short duration of illness and younger age both predict better outcomes in FT-AN (Eisler et al., 2000; Lock et al., 2006; Agras et al., 2014) as does a lower level of emaciation at the start of treatment (Eisler et al., 2000). Adolescents with lower levels of eating disorder pathology had higher rates of recovery in studies by Eisler et al., (2000) and Agras et al., (2014). In the latter study, intact families and adolescents without binge-purge symptoms also fared better.

In RCTs for $\mathrm{BN}$, the following have been found to predict higher rates of abstinence at end of treatment: being male, milder eating pathology, lower baseline depression scores and higher family cohesion (Le Grange et al., 2015; Le Grange, Crosby and Lock, 2008).

\section{$\underline{\text { 2.6.2 Moderators }}$}

Our understanding of moderators and mediators is necessarily limited to those variables that have been chosen for data collection in published RCTs. Unsurprisingly, adolescent eating pathology has been one of the most commonly used measures in the 
field. One particular aspect of eating pathology - eating-disorder related obsessionality - has frequently emerged as a moderator of treatment in FT-AN. For adolescents with high obsessionality, outcomes have been better when the family have been offered a longer course of treatment (Lock et al., 2006). Conjoint treatment also appears more helpful for this group of patients, as compared to individual therapy (Lock et al., 2010) or separated forms of FT-AN (Eisler et al., 2000; Le Grange et al., 2016). However, adolescents with high obsessionality benefitted less from FBT as compared with SyFT, a more generic manualized FT approach (Agras et al., 2014). Since adolescents with high obsessionality had higher baseline levels of eating pathology, depression, anxiety, and compensatory behaviors, the findings suggest that a broader treatment focus within family therapy may be beneficial for adolescents with high levels of comorbidity.

Other findings on moderation are that adolescents with AN with binge-purge symptoms benefitted more from a longer course of FBT, as did non-intact families (Lock et al., 2006). In BN, participants with lower family conflict and lower eating pathology scores responded better to FBT-BN compared to CBT (Le Grange et al., 2015; Le Grange, Crosby and Lock, 2008).

The possible role of EE as a moderator of treatment effectiveness in FT-AN has received particular attention over the years. Pilot studies of FT-AN found that a high level of EE (particularly maternal criticism) towards the adolescent was highly predictive of poor engagement in family therapy (Szmukler et al. 1985) and poor treatment outcome (Le Grange et al., 1992; Dare et al., 1995). As previously discussed, Eisler et al. (2007) found that high EE at baseline predicted poorer outcomes at five- 
year follow-up for those in conjoint family therapy. More recently, Rienecke et al. (2016) found that patients with mothers rated high in hostility by observers gained more weight in individual therapy than FBT. Furthermore, higher paternal criticism is associated with poorer outcomes regardless of treatment (Rienecke et al., 2016; Le Grange et al., 2016).

Forsberg et al. (2015) have suggested that the inconsistent findings on EE might reflect an underlying third variable. We have suggested elsewhere (Jewell et al., 2016) that a concept such as attachment, which overlaps theoretically and empirically with that of EE (Scott et al., 2011; Green et al., 2007), may help to explain the differential response to FT-AN. The ability to tolerate strong negative affects is a marker for secure attachment (Fonagy et al., 2012). Some parents and adolescents with insecure attachment representations may therefore have a lower threshold for tolerating the emotional arousal that is likely engendered in the early weeks of FT-AN. If adolescents and/or parents become highly emotionally aroused during family therapy sessions, they may be more likely to interpret material arising from the session - such as comments by family members - in negative terms, such as criticism or blame. This may also be the case during emotionally charged interactions in the family home, particularly family mealtimes, making the task of helping the young person increase their food intake more demanding. More fundamentally, the meaning given to the parental task of managing their child's eating may differ according to family members' attachment representations. Adolescents with secure attachment may be more likely to accept parental supervision of their eating as an act of care. By contrast, adolescents with a preoccupied attachment style (Shmueli-Goetz et al., 2008), who tend to be caught up in past grievances, may 'push back' at such parental supervision 
experiencing it not as caring but controlling and nagging. Similarly, parents who themselves have unresolved attachment issues are more likely to lack confidence in themselves as parents (Jones et al. 2015) and may respond to their child's rejection of help by increasing their own negativity. In such cases, conceptualizing the therapeutic task as 'putting parents in control' may in fact be counter-productive.

\subsubsection{Potential mediators of treatment}

Currently, no formal mediators have been identified in studies of eating disorder focused family therapy. This is a serious barrier to improving treatment, since an understanding of mediators could inform adaptations to the model, allowing for active components to be intensified whilst redundant elements could be discarded (Kazdin and Weisz, 1998).

What does the available evidence suggest might be a plausible mediator? Several lines of research provide support for the importance of parental variables. In a study by Ellison et al. (2012), clinicians rated parents on variables which are seen as key to change in the Lock et al. (2001) treatment manual, such as parental control and unity, in a sample of 59 adolescents receiving FBT. Higher scores on these variables predicted adolescent weight gain, with the strongest predictor being parental sense of being in control over AN. However, change in these variables across time was not assessed. By contrast, Robinson et al. (2013) found that parental self-efficacy increased over the course of FBT, and that increases in parental self-efficacy over treatment were correlated with reductions in adolescent eating pathology. From a clinician perspective, parental empowerment is also seen as key ingredient of FBT 
(Dimitropoulos et al., 2015), although this might be seen as somewhat circular in that empowerment is a key theme within the treatment model.

Two studies have illuminated change processes in MFT-AN using qualitative data. Engman-Bredvik et al. (2015) interviewed 12 parents, and reported that parents valued the role of the group in reducing parents' perceptions of blame and stigma arising from having a child with AN. Parents also spoke of gaining increased competence in their parental roles, which was attributed to learning gained through meeting other parents. Similar themes emerged from Voriadiki et al.'s (2015) study of parents and adolescents who attended MFT-AN. Key themes included the importance of feeling less alone, and more hopeful about recovery, as a consequence of meeting other families. Adolescents reported becoming more accepting of the idea that they had a problem. These findings suggest that, at least in MFT-AN, change is happening rapidly, and that the development of hope - a crucial common factor - appears to be mobilized by the treatment context.

Indeed, one of the challenges for FT-AN research is that change can take place very rapidly when the approach is successful. Weight gain achieved by the fourth treatment session - usually one month after the start of treatment - is a predictor of good outcome at end-of-treatment (Doyle et al., 2010; Le Grange et al., 2014). Similarly, in FBT$\mathrm{BN}$, early change in binge eating and purging among adolescents is a good predictor of response at the end of treatment (Le Grange et al., 2008). This has important implications in the search for treatment mediators. Since by definition a mediator must change value following the start of treatment (Kraemer et al., 2002), mediators may be operating very early in treatment. Thus, a challenge for future research is both to 
conceptualize what changes, and to capture it empirically. Variables such as parental self-efficacy may operate as mediators in some families but not others, and it is plausible that change mechanisms may be different in families who respond early to treatment as compared with families where change takes longer to achieve. To explore these issues it will be necessary to conduct process studies using multiple time-points for data collection, particularly within the early phase of treatment. A further hypothesis is that the first assessment appointment may be a key precipitant of change for some families. If true, future research could measure expectancy effects by asking families to evaluate their views on attending the service, and hopes for recovery, prior to attending the first appointment.

\subsubsection{Therapeutic alliance}

The role of the therapeutic alliance in psychotherapy outcomes has perhaps received more attention than any other common factor. However, findings on alliance in FTAN thus far do not tell a simple story partly because different measures have been used in different studies including both observational and self-report measures and partly because adolescent and parent alliance ratings appear to predict different aspects of outcome. Isserlin \& Couturier (2012) using an observational measure of alliance found that parental alliance predicted engagement in treatment and early behavioral change, whereas adolescent alliance was positively linked to remission in eating disorder cognitions at the end of treatment. Similar findings using a different observational measure of alliance are reported by Pereira et al., (2006) although in this case adolescent alliance predicted both psychological change and early weight gain, whereas parental alliance predicted engagement in treatment. Ellison et al., (2012) also report a positive association between parental alliance and post-treatment weight gain, and a negative association with dropout. Somewhat surprisingly the study found 
differences between maternal and paternal alliance ratings, with maternal alliance predicting greater weight gain and lower dropout, and paternal alliance predicting less weight gain.

Complex findings have also emerged from two studies of alliance using data from Lock et al.'s (2010) study of FBT vs. individual therapy. In the first study, observerrated alliance predicted partial, although not full remission, at end-of-treatment, for both FBT and individual therapy (Forsberg et al., 2013). In a more recent study, Forsberg et al. (2014) found no association between parental alliance rated at session 4 and remission at end of treatment. Given the previously discussed finding that many adolescents achieve early weight gain, it is hard to unpick the temporal ordering of alliance and weight gain; it is certainly plausible that weight gain itself will have a positive impact on alliance and disentangling the impact of alliance on outcome may therefore be difficult.

The mixed findings on alliance and outcomes in FT-AN point to a potentially complex relationship between these variables. If our hypothesis that meaningful change in FTAN may happen as early as the first assessment session, and that common factors such as hope/expectancy effects contribute to this, then perhaps the alliance to an individual clinician may need to be considered alongside of the impact of the service context on the family members' views - particularly their trust or belief in the treatment center as a credible institution. A useful conceptualization is offered by Fonagy and Allison (2014), who have applied the notion of epistemic trust to the process of psychotherapy. Epistemic trust refers to an individual's willingness to consider new knowledge from another person as trustworthy, generalizable, and relevant to the self (Fonagy and 
Allison, 2014), and thus draws attention to the relational context in which learning takes place (Landrum, Eaves and Shafto, 2015).

The expert multidisciplinary team context in which the initial assessment takes place may play a key role in enhancing the developing alliance with the therapist providing a safe base for treatment and promoting the development of epistemic trust. The knowledge that the team as a whole have of the nature of eating disorders and the way they impact family life resonates with the family's experiences and gives them a sense of being understood and supported. This contributes to the perceived credibility and trustworthiness of the therapist and supports the development of the therapeutic alliance. However, the development of epistemic trust may not proceed in a straightforward fashion in all cases; insecure attachment and emotion regulation difficulties in particular may mitigate against the development of such trust, at least in part due to the hypothesis proposed earlier in this paper. If epistemic trust is a precondition for learning, then this may be a useful organizing principle for treatment. Specific interventions, such as therapists coaching parents during the family meal, may succeed or fail based on the extent to which trust has been developed. For families who do not develop such trust early in treatment, it may be fruitful for the therapist to consider alternative ways by which this may be achieved. Viewed from this perspective, seeing parents and adolescents separately, or offering a multi-family therapy group, provide new contexts for the development of epistemic trust and change.

\subsection{Discussion}

Eating disorder-focused family therapy has become firmly established as an empirically supported treatment, with evidence of superior efficacy relative to 
individual approaches in both AN and BN (Watson and Bulik, 2013; Couturier et al., 2013a; Le Grange et al., 2015). Nevertheless, caution is needed here. Whilst there is evidence of treatment efficacy from well-designed RCTs, to date there have been no replication studies conducted independently of model developers. As a result, it is possible that allegiance effects may account for the apparent superiority of family therapy. Moreover, other potentially viable treatments for adolescent anorexia nervosa such as CBT (Dalle Grave et al., 2013) have not been compared directly with FT-AN, and it is possible that they could be equally effective. It is important to note that even in comparisons of family versus individual approaches, the treatments often have a great deal in common with each other. Parental involvement has been described as a sine qua non of child and adolescent eating disorders treatment (Lask, 2000). In line with this view, most of the 'individual' treatments that have been studied included at least some collateral parent sessions. This means that parents still had the benefit of the expectancy effects that might accrue both from seeing a therapist who presents a clear model of treatment, and also through contact with other professionals such as pediatricians. Given that a treatment which excluded parents completely would be in many instances ethically problematic, and unacceptable to many families, it may be fruitless to frame the debate in terms of family and individual approaches being in opposition to each other. While from a research point of view it is perfectly legitimate to ask questions about the relative efficacy of each treatment, in clinical practice treatments will often be combined and family and individual therapy will then be seen as complimentary components of treatment.

In clinical settings outside of an RCT, children and adolescents will often receive individual time with therapists quite routinely. In FT-BN, individual sessions are built 
into the treatment model, which makes use of elements drawn from CBT. Similarly, in both MFT-AN and MFT-BN, group sessions for adolescents on their own make up a significant proportion of the therapeutic program. However, FT-AN currently lags behind in its conceptualization of when adolescents should be seen on their own, or what the therapeutic aims of individual therapy should be. For families who currently do not respond to treatment, adaptations to FT-AN, potentially involving separated adolescent and parent sessions, are in need of theoretical elaboration and empirical validation. Meanwhile, for adolescents with co-morbidities, the third stage of FT-AN (once weight restoration is well underway) may constitute an ideal time in which to add adjunctive treatments, such as CBT. For both adolescent $\mathrm{AN}$ and BN, the key questions for the field are: For whom does the current treatment work? What alternatives or additions should be offered for those who do not benefit? And can the treatment model be improved so that adolescents achieve higher rates of remission?

\subsection{Conclusion}

Based on the available evidence, we can conclude that the most successful treatments of child and adolescent eating disorders have the following general features in common; they:

- actively mobilize the family as a key treatment resource to promote changes in eating disorder behaviors early on in treatment;

- provide a coherent model of treatment (ideally operationalized in a treatment manual) that allows a degree of consistency in the way treatment is provided, while providing enough flexibility to tailor the treatment to the specific needs of individual families;

- are delivered by clinicians with significant expertise in eating disorders, where possible within a specialist multidisciplinary team context; this provides a setting 
which engenders a sense of safety and trust in which adolescents and parents can take on new learning and new behaviors.

Although the existing treatment manuals specify the ingredients that should form the content and process of treatment, the empirical evidence to support our understanding of which ingredients are necessary and how they bring about change is still limited. Developing an understanding of the mechanisms of change and the factors that moderate how these operate in different individuals and different families is therefore a key priority for research.

As many have argued previously, improving our understanding of moderators and mediators signposts when and how treatments need to be modified, or what additional or alternative therapies might need to be offered when the standard treatment is not sufficient. They also, however, offer a new perspective on the debate about the role of common factors in therapy. Some common factors, such as temperament, may not be amenable to change but interact with the specific treatment factors and therefore act as moderators. Others, such as therapeutic alliance, may both interact with specific treatment factors and be amenable to change and can be understood as part of what mediates change. We suggest that a moderate common factors position - both seeking to understand the commonalities between treatments, and yet open to the idea that different treatment approaches can have distinctive effects, and may also interact in specific ways with different common factors - offers a coherent base from which to begin to disentangle these important and complex issues. 


\subsection{Updated literature review}

\subsubsection{Randomised controlled trials}

Since the publication of the narrative review presented in this chapter, there have been few major changes to the evidence base. Two studies employing RCT designs have been reported, albeit neither are rigorously conducted efficacy trials. Herscovici et al. (2017) conducted a small, exploratory study $(n=23)$ comparing FT-AN with and without a family meal. Unsurprisingly, given the sample size, no differences emerged between the two groups. Nyman-Carlsson et al. (2020) reported a comparison of individual CBT versus family therapy in a sample of 78 young adults. The family therapy model was based on FT-AN but adapted by the study team. Likewise, the CBT offered was similar to the enhanced CBT (CBT-E) model for eating disorders developed by Fairburn (2008), but adapted by study authors. There were no differences between treatment groups on any outcome measures, which included body mass index, eating disorder psychopathology and diagnostic status; end of treatment was at 18 months, with follow-up 18 months after that. Whilst this lack of difference between treatments in keeping with the common factors view described earlier in the chapter, the study is underpowered and thus conclusions about common versus specific factors cannot be drawn. Predictors of outcome were reported in a separate study (Nyman-Carlsson et al., 2019). In the family therapy group, lower interoceptive deficits predicted higher weight gain, whilst bulimic behaviour and emotion dysregulation predicted increased scores on a diagnostic index comprised of questions relating to fear of weight gain, body image and eating habits. Conversely, in the CBT group higher interoceptive deficits and lower emotion regulation difficulties predicted greater weight gain. 
A non-randomised effectiveness trial in adolescent eating disorders has recently been reported by Le Grange et al. (2020). This is the first study to compare FBT (Lock et al., 2001) with CBT-E (Fairburn, 2008). In the CBT-E arm, therapists used the transdiagnostic CBT-E treatment manual which was adapted for adolescents (Dalle Grave \& Calugi, 2020), whilst in the FBT arm, therapists used either the FBT-AN (Lock et al., 2001) or FBT-BN (Le Grange \& Lock, 2007) manuals. The overall sample comprised 97 adolescents with predominantly restrictive eating disorders, who were stratified into two groups: a lower weight group with a percentage median Body Mass Index $(\% \mathrm{mBMI})$ of $<90 \%$, and a higher weight group of over 90\%mBMI. Patients and their families could choose between treatments, resulting in significantly older patients, with a longer duration of illness, in the CBT-E group. Lengths of treatment also differed in the low weight group, where families received up to 20 sessions of FBT over approximately 6 months but received up to 40 sessions of CBT-E over 9-12 months.

The main finding of the study was that the slope of weight gain between the beginning and end of treatment was significantly higher in the FBT than CBT-E arms of the study, for both lower and higher weight groups. Several potential moderators are reported for weight gain at end of treatment, including: patients with a higher level of depression having CBT-E gained weight more slowly, whereas depression level did not affect weight gain in FBT; and patients with a history of psychiatric hospitalization lost weight in CBT-E, whereas patients without such a history gained weight in either CBT-E or FBT. Non-specific predictors of outcome included a history of abuse, which predicted lower weight gain in both the lower and higher weight groups. In the lower weight cohort, a higher rate of weight gain was 
predicted by less externalizing problems, absence of comorbid psychiatric disorder and lack of prior mental health treatment. However, analyses from this study should be treated with extreme caution due to methodological weaknesses including lack of statistical power and missing data leading to sampling bias. For example, in the lower weight cohort, the CBT-E sample comprised $n=16$, but at end of treatment only $\mathrm{n}=9$ were assessed, and at 12-month follow-up only 5 were assessed; meanwhile, the FBT sample comprised $n=21$ of whom only 14 were assessed at end of treatment, and 9 assessed at follow-up. No missing data were imputed, meaning that analyses were based on very small samples with a high rate of attrition.

\subsubsection{Reviews}

Arguably the most important publications since 2016 have been reviews. Prior to these recent reviews, one of the few meta-analytic studies was conducted by Couturier, Kimber, and Szatmari (2013a), which combined both FT-AN and FT-BN studies and concluded that family therapy demonstrated superiority to individual approaches at follow-up, but not at end of treatment. The picture emerging from several recent meta-analyses has been less favourable to FT-AN, although it is important to clarify the differences in methodology that help to explain the different conclusions.

The National Institute for Health and Care Excellence (NICE) published national guidelines for the identification and treatment of eating disorders for the United Kingdom in 2017. Whilst NICE (2017) recommended FT-AN as the first-line treatment for adolescent anorexia nervosa, their meta-analysis of treatment studies did not provide evidence of superior efficacy of FT-AN compared with alternative treatments. Unlike Couturier, Kimber, and Szatmari (2013a), NICE ran separate 
analyses for AN and BN. The most relevant analyses for AN included three studies that compared FT-AN against individual therapy approaches: Lock et al. (2010), Robin et al. (1999) and Russell et al. (1987) (with follow up for the latter study reported in Eisler et al., 1997). The analyses found no benefit of FT-AN on remission at end of treatment or follow-up, although FT-AN was superior to individual therapy; at end of treatment, there were benefits of FT-AN as compared with individual therapy on Morgan-Russell outcome, eating pathology and weight outcomes. However, due to low quality ratings of the studies, NICE gave the weaker recommendation to 'consider' rather than 'offer' family therapy for adolescent AN. By contrast, the stronger recommendation to 'offer' family therapy was made by NICE for adolescent BN, on the basis of their meta-analysis of just two FT-BN studies (Le Grange et al., 2015; Schmidt et al., 2007) showing superior remission compared to individual approaches as follow-up, but not remission.

Couturier et al. (2020) recently reported new Canadian practice guidelines for the treatment of child and adolescent eating disorders. New meta-analyses were not conducted for their review, but study quality was assessed. These guidelines state that FBT has the most evidence to support its use in child and adolescent eating disorders, and they give a 'strong recommendation' in favour of FBT for any child or adolescent with AN or BN, especially for those who have been ill less than 3 years. The authors call for more research in the following areas: treatments for complex presentations of eating disorders, including patients with complex co-morbidity including borderline personality; research on factors influencing likely treatment response; the development of new and adjunctive treatments for families unable to engage in FBT; and investigation of how treatment needs may differ for particular 
populations including individuals with non-binary gender identities. These recommendations have some areas of overlap with the research recommendations given by NICE (2017), who also called for research into eating disorder populations with comorbidities, such as Type 1 diabetes. NICE call for RCTs to be conducted, and for these to include investigation of moderators and mediators. NICE also call for research into the factors associated with staying well after successful treatment of AN.

The Cochrane review conducted by Fisher et al. (2019) on family therapy approaches for anorexia nervosa included a number of meta-analyses, although differed from NICE in the choice of studies included in their analyses. The headline conclusion of the review was that there was low-quality evidence to support the efficacy of family therapy compared to treatment as usual in terms of remission posttreatment (based on two studies, with 81 participants), but not at follow-up. However, the two studies used for the analysis were both with adults. The analyses of greater relevance to FT-AN were the comparisons of family therapy to psychological interventions, which also included the Lock et al. (2010), Robin et al. (1999) and Russell et al. (1987) trials, but also included adult studies by Dare et al. (2001) and Crisp et al. (1991). Fisher et al. (2019) found no evidence of superiority of family therapy relative to psychological interventions in terms of remission; however, there were superior outcomes for FT-AN for weight at end of treatment, although not at follow-up, which is in keeping with the findings by NICE (2017) and the meta-analysis by Murray et al. (2019) which is discussed below. The authors comment on the high risk of bias found in the included studies, for instance through outcome assessment being conducted by clinicians rather than blinded assessors. The 
review ends with a recommendation for well-designed trials of family therapy versus other psychological approaches using standard outcomes; such studies should investigate the impact of both chronicity and age.

A meta-analysis completed by Murray et al. (2019) of treatment studies of AN across the age range highlights the discrepancy between weight and psychological symptom recovery in existing treatments. The study analyses weight and psychological symptom outcomes at end of treatment and follow-up for AN treatment studies that employed RCT designs comparing an intervention with a comparator. The main finding was that $\mathrm{AN}$ treatments demonstrated superiority to comparator treatments for weight outcomes only at end of treatment, and not at follow-up; there was no evidence of superiority of AN interventions for psychological symptoms. In the secondary analyses, there was a moderating effect of treatment type, with smaller effects for psychosocial treatments relative to other treatment categories, but the summary effect size for family therapy treatments remained significant.

Given that many of the RCTs conducted in the FT-AN field compare two slightly different formats of the same treatment, it is perhaps unsurprising that few differences between treatments emerged - particularly from a common factors perspective. However, the authors ran a further analysis in which they excluded comparisons of different doses of the same treatment or nuanced forms of the same treatment, and this analysis yielded similar results. Whilst one interpretation of this meta-analysis is that the AN field needs new treatments that focus on reducing psychological symptoms, another view is that change in such symptoms may take longer than the typical time frames employed in RCTs - for instance, in Accurso et 
al.'s (2020) study of parental views on their child's recovery, psychological symptom recovery was estimated to take 3.9 years on average (see further discussion in Chapter 6). Whether such symptom recovery can be expedited in new, more focused treatments is an empirical question requiring further research.

Finally, an important systematic review of moderators and mediators in FT-AN was conducted by Hamadi \& Holliday (2020), who found that it was not possible to draw firm conclusions about the topic. They helpfully draw attention to weaknesses in research methodology used in this area, including the use of secondary analyses of trial data to investigate moderators and mediators without sufficient statistical power; furthermore, they assert that conducting post-hoc analyses involves the risk of 'data mining'. Of the studies they include that were not part of our earlier narrative review, the study by Sadeh-Sharvit et al. (2018) is noteworthy since it investigated selfefficacy as a mediator, using data from the Agras et al. (2014) trial comparing FBT with Systemic Family Therapy. The study found that increases in maternal selfefficacy by session 8 mediated weight gain by session 10 for participants in the FBT arm of the trial. As noted by Hamadi and Holliday (2020), parental self-efficacy shows promise as a possible mediator in FBT, although currently there is a need for more robust research to investigate this.

\subsubsection{Empirical studies}

Notwithstanding the methodological limitations of such studies, several notable secondary analysis studies of trials have been published since 2016. Allan et al. (2018) report on change in EE in the two arms of the Le Grange et al. (2016) RCT comparing parent-focussed therapy (PFT) versus conjoint Family-Based Treatment 
(FBT). The authors found that PFT was associated with a decrease in maternal criticism regardless of treatment outcome, whereas FBT was associated with increases in maternal criticism. This finding adds further evidence that the format of family therapy delivered can have tangible and potentially negative impacts on some families. However, as discussed further by Simic, Jewell and Eisler (2020), there appear to be risks and benefits to conjoint or separated treatment. In clinical practice, clinicians are likely to make use of both formats, as advocated by Dare and Eisler (1997). Nevertheless, Allan et al.'s (2018) study points to the potential need for therapists to regard $\mathrm{EE}$, and increases in $\mathrm{EE}$ over time, as one important source of information to guide decisions about the format of therapy.

In another secondary analysis of this trial, Hughes et al. (2019) replicated past findings that early weight gain predicted remission, and also explored the predictors of early response in terms of weight recovery. For adolescents in the FBT arm, early responders had fathers with significantly higher therapeutic alliance and lower criticism compared with early non-responders. Several variables predicted early response in PFT, including lower criticism in mothers, higher adolescent-rated expectancy about the success of treatment, and higher adolescent-rated therapeutic alliance with their nurse.

Another study of particular relevance to this thesis is that by Wallis et al. (2017b), who report an analysis of data from the trial by Madden et al. (2015), which compared outcomes following shorter or longer inpatient stays followed by FBT ( $\mathrm{n}=$ 82). In this secondary analysis, Wallis et al. (2017b) report psychological predictors of outcome in a sub-set of participants $(n=57)$ who had completed the treatment 
protocol and had available outcome data. Participants from both treatment arms were collapsed into one group. Attachment was assessed in adolescents using the Inventory of Parent and Peer Attachment (Armsden \& Greenberg, 1987), a selfreport measure (see Chapter 4); family functioning was rated with the Family Assessment Device (FAD) (Epstein et al., 1983), also a self-report measure. Neither adolescent attachment at baseline, nor change in attachment, were associated with remission at 20-sessions or at 12-month follow-up. At baseline, better adolescent reported problem-solving, communication and general functioning, as assessed using the FAD, were associated with remission at session 20, but not at 12-month followup. No mother-reported scales on the FAD were associated with outcome at either timepoint, whereas Behavioural Control as rated by father at the start of treatment predicted remission at 12-month follow-up, though not at end of treatment. Wallis et al. (2017a) have also published an important qualitative study of change in familial relationships over time, as discussed in Chapter 1 of this thesis.

\subsubsection{Summary of the updated literature}

Recent years have yielded little progress towards the goals of developing a better understanding of predictors of outcome or mechanisms of change in FT-AN. At the same time, several important reviews have brought into relief the limitations of the existing evidence base. Whilst meta-analytic evidence does point to the superior efficacy of FT-AN in terms of weight outcomes at end of treatment, this is based on a small number of studies. Meanwhile, evidence regarding predictors of treatment and mechanisms of change in FT-AN remains scant. In terms of theory, there is scope to research new concepts and to investigate the interplay between common and specific factors in FT-AN. In the next chapter, we consider the concepts of 
attachment and mentalization and their possible relevance to child and adolescent eating pathology. 
Chapter 3 - Attachment and mentalization and their association with child and adolescent eating pathology: A systematic review

This chapter is presented as a published journal article. Other than the updated literature review at the end, it is an exact copy of the following publication:

Jewell, T., Collyer, H., Gardner, T., Tchanturia, K., Fonagy, P., Simic, M. and Eisler, I. (2016). Attachment and mentalization and their association with child and adolescent eating pathology: A systematic review. International Journal of Eating Disorders, 49:354-373. 


\subsection{Abstract}

Objective: Insecure attachment and mentalizing difficulties have been associated with eating pathology in adulthood. However, it is unclear whether eating pathology is associated with attachment or mentalization in children. The aim of this study is to systematically review the literature in this emerging field.

Method: Electronic databases were used to search for articles.

Results: 22 studies were identified. In the 15 studies investigating attachment, an association with eating pathology was found in all studies. Mentalizing difficulties and eating pathology were found to be correlated in the seven studies which examined their association.

Discussion: In keeping with the adult literature, cross-sectional studies of children and adolescents consistently report associations with eating pathology. There is some evidence from prospective studies that insecure attachment may be a risk factor for the development of eating pathology in adolescence. The literature on mentalization and eating pathology suggests that adolescents with anorexia nervosa may have difficulties in recognizing emotions. Further research utilizing clinical samples and well-validated measures of attachment and mentalization are required to shed further light on this area. 


\subsection{Introduction}

In recent years the concepts of attachment and mentalization have attracted increasing research interest in the field of eating disorders (Caglar-Nazali et al., 2014; Kuipers \& Bekker, 2012; Tasca \& Balfour, 2014). An association between attachment insecurity and eating disorders has been a consistent finding of numerous studies over the past quarter century (Armstrong \& Roth, 1989; Ringer \& Crittenden, 2007; Ward et al., 2000). Meanwhile, several studies have reported findings of mentalizing difficulties in adults with eating disorders (Morris et al., 2014; Oldershaw et al., 2011; RothschildYakar et al., 2010a; Russell et al., 2009). Attachment and mentalization represent two aspects of social and emotional development which have been considered to be salient to eating disorders, either as foci for treatment, or through inclusion in causal models (Caglar-Nazali et al., 2014; Connan et al., 2003). Whilst the two concepts are distinct, there are areas of both theoretical and empirical overlap. Importantly, both attachment and mentalization are developmental processes, which appear to be in a state of flux during the adolescent period.

The number of studies which have examined attachment and mentalization in adolescent samples is relatively small, and no systematic review has been conducted in this area. The primary aim of this paper is therefore to systematically review the literature on attachment and mentalization as it relates to child and adolescent eating pathology. The secondary aim is to consider the implications of the evidence in relation to etiological models for eating disorders. 


\subsubsection{Attachment}

Bowlby (1979) proposed attachment as a theory of human behavior and relationships across the lifespan. He suggested that infants develop mental representations of the self and others in response to their repeated experiences of the availability and responsiveness of their caregivers. These 'internal working models' (Bowlby, 1969) (IWMs) were postulated to form a lasting template for appraisals of self and others in close relationships across the life-course.

The evidence for stability in attachment representations across the lifespan is complex. Pinquart et al.'s (2013) meta-analysis of longitudinal studies of attachment from infancy to adulthood found no significant stability in attachment classification in intervals over 15 years. However, levels of stability were greater in lower-risk samples. Possible reasons for the lack of attachment stability across time include difficulties around measurement, and changes in caregiver quality over time leading to re-appraisal of IWMs. More recently, a twin study by Fearon et al. (2014) showed a strong genetic influence on attachment representations in adolescence. The development of adult attachment representations therefore appears to be a complex process, with significant amounts of change across developmental periods, and a role for genes as well as environment.

In adults, attachment can be assessed by interview or self-report. The Adult Attachment Interview (George et al., 1985) (AAI) yields four categories of attachment representations, labelled secure, dismissive, preoccupied and disorganized. An alternative approach to assessment has been the use of self-report measures of 
attachment style, which assess thoughts and behavior in relation to adult romantic relationships. Brennan, Clark and Shaver (1998) found two factors to underlie all the extant adult self-report measures included in their meta-analysis. Attachment anxiety relates to an attachment style in which an individual is highly motivated to engage in close relationships and has a tendency to idealize others while devaluing themselves. By contrast, individuals with attachment avoidance tend to minimize their own attachment needs and avoid close relationships with others.

The measurement of attachment in middle childhood and adolescence is complex, and there is an absence of a widely agreed 'gold standard' measure. However, findings increasingly support the use of interview-based measures of attachment for adolescents (Scott et al., 2011) such as the Child Attachment Interview (ShmueliGoetz et al., 2008; Venta et al., 2014). A small number of well-validated self-report measures of attachment for children and adolescents have been developed (Armsden \& Greenberg, 1987; Kerns et al., 1996), but many of the measures for this age group have only limited data on validity (Kerns, 2008).

In adult samples of patients with eating disorders, higher rates of insecure attachment have been consistently found compared with rates in community samples (Kuipers \& Bekker, 2012; Tasca et al., 2011; Zachrisson \& Skårderud, 2010). Many studies have used cross-sectional study designs to examine associations between attachment security and eating pathology, using either self-report (Armstrong \& Roth, 1989; Broberg et al., 2001) or interview-based measures of attachment (Barone \& Guiducci, 2009; Ringer \& Crittenden, 2007; Ward et al., 2000). Several more recent studies have 
explored potential mediators of the relationship between attachment and eating pathology (see Tasca \& Balfour, 2014 for a review). For example, both Tasca et al. (2009) and Ty and Francis (2013) have reported affect regulation to be one such mediator of the relationship. Finally, a small number of studies have begun to examine the role of attachment in the treatment process for adults with eating disorders. For instance, Illing et al. (2010) found that higher attachment anxiety was significantly related to greater severity of eating disorder symptoms and poorer treatment outcome in a clinical sample with a range of eating disorder diagnoses.

\section{$\underline{\text { 3.2.2 Mentalization }}$}

Mentalization refers to the capacity to reflect and interpret one's own and others' behavior in terms of intentional internal mental states such as thoughts, feelings, and beliefs (Fonagy \& Target, 2006). The concept thus includes, but is not limited to, the concept of theory of mind (TOM) (Ha et al., 2013). The concepts of attachment and mentalization are at their closest point theoretically within the developmental model proposed by Fonagy et al. (2002), who postulated that mentalization develops within the social matrix of attachment relationships.

The links between attachment and mentalization have been explored empirically with adults using the concept of reflective function (RF). This refers to the ability to reflect on the mind of self and others in the context of attachment relationships (Fonagy et al., 1991). RF can be rated from AAI transcripts using the Reflective Function Scale (Fonagy et al., 1998), and a small body of research has examined RF in eating disorder samples. 
As compared with community controls, patients with anorexia nervosa were found to have lower RF - meaning poorer mentalization - in studies by Ward et al. (2001) and Rothschild-Yakar et al. (2010). Fonagy et al. (1996) also reported lower RF in a sample of adults with eating disorders, although the diagnostic profile of the sample were not specified. Rothschild-Yakar et al. (2010) found that higher RF was associated with lower drive for thinness in their community sample. However, in their clinical sample, which consisted of inpatients with anorexia nervosa binge/purge subtype, no correlation was found between mentalizing and drive for thinness, and a positive correlation was found between mentalization and bulimic symptomology. A complex relationship between mentalization and bulimia is also suggested by Pedersen et al.'s (2012) study, in which no difference in mean RF scores was found between a sample of women with bulimia nervosa and healthy controls. However, patients with bulimia were more polarized in their RF abilities, with more scores in both the low and high range.

Other researchers have investigated the concept of TOM within eating disorders, with a recent systematic review concluding that patients with eating disorders have a poorer understanding of the mental states of others, as compared with controls (Caglar-Nazali et al., 2014). For example, Harrison et al. (2009) found that adult women with anorexia nervosa (AN) had significantly lower scores on the Reading the Mind in the Eyes task (Baron-Cohen et al., 2001) as compared with a clinical comparison group. However, Oldershaw et al. (2010) found almost complete normalization of emotion recognition ability and emotional TOM in patients who had recovered from AN, suggesting that impairments in these domains may be a consequence of starvation. 


\subsection{Rationale for this review}

Attachment and mentalization represent two areas of social and emotional functioning that are salient in adult eating disorders, and findings support their inclusion in explanatory models both as causal and maintaining factors (Caglar-Nazali et al., 2014). However, the existing research on both attachment and mentalization is beset by a number of limitations. Firstly, studies using clinical samples may be biased by study designs in which chronically unwell patients are recruited to studies using nonrandom sampling methods. Secondly, studies have not always adequately controlled for potential confounders, such as depression or chronicity. Thirdly, the crosssectional study designs used in the adult literature do not provide strong evidence about the role of either attachment or mentalization in the etiology of eating disorders. Moreover, the relationship between attachment and mentalization is unclear and likely to be complex. From a psychoanalytic perspective, both Bruch (1973) and Fonagy et al. (2002) postulated a role for misattuned early attachment relationships in the development of mentalizing difficulties in patients with eating disorder. Empirically, there is evidence that RF predicts attachment status (Bouchard et al., 2008). However, mentalizing difficulties such as impairments in TOM have also been conceptualized as intermediate phenotypes that are shared between eating disorders and autism spectrum disorders (see Treasure, 2013 for a review). A review of studies of child and adolescent eating pathology and its association with attachment and mentalization therefore has the potential to shed light on etiological models of eating disorders.

A second rationale for this review is that there is evidence to suggest that attachment (Illing et al., 2010b; Tasca \& Balfour, 2014a) and mentalizing (Rothschild-Yakar et al., 2010a; Schulte-Rüther et al., 2012a; Wentz et al., 2009) may have a bearing on 
treatment outcome in eating disorders. It has been suggested that subgroups of eating disorder patients that differ in symptom profile, attachment classification and mentalizing abilities might require a different therapeutic approach (Kuipers \& Bekker, 2012; Tasca \& Balfour, 2014). These considerations are likely to be salient in the treatment of adolescent eating disorders, not least because both attachment representations and mentalizing capacity appear to be in a state of flux during adolescence (Bleiberg, 2013). Indeed, emerging evidence from brain imaging studies (e.g. Blakemore, 2008) suggests that adolescence may be a critical stage in the development of mentalizing and social cognition. Furthermore, changes in attachment and mentalizing over the course of therapy have been shown to mediate treatment response in adolescents who self-harm (Rossouw \& Fonagy, 2012), raising the possibility that attachment and mentalizing may be worthwhile treatment targets in other adolescent clinical populations, such as those with eating disorders.

\subsection{Method}

A systematic review of studies examining attachment and/or mentalization with children and/or adolescents with eating pathology was conducted using the Medline (1946-Present), PsychInfo (1806-Present) and Embase (1974-Present) databases. The search was conducted according to PRISMA guidelines (Moher et al., 2009). We searched for relevant articles in English using the keywords attachment, mentalization, social cognition, theory of mind, reflective function, eating disorders, eating pathology, anorexia nervosa, bulimia nervosa and binge eating, up until May 2015. Limits were set to exclude studies of infants, and to exclude dissertations. Referencechecking of located articles was conducted. Searches were conducted independently 
by HC and TG. Study eligibility was assessed by TJ. In cases where eligibility was unclear, decisions on inclusion/exclusion were made by TJ and IE.

Studies were included if they used a measure of eating pathology and also a measure of attachment or mentalization. The upper age limit for inclusion was set at 20 . There is no agreed definition of adolescence, with chronological age being just one of several ways to define it (Hawton et al., 2012). We were guided by the World Health Organization's definition of adolescence as ending at age 19 (World Health Organization, 2014). In order to include studies of 19-year-olds, we decided on 20 as the upper age limit. Data on age range, mean age and standard deviation are presented in Tables 1 and 2. For studies which did not present a full range of data on age, we made a judgement based on the available data and sampling method to determine inclusion. Case studies and studies of obesity were excluded.

\subsubsection{Assessment of bias}

We assessed bias within individual studies using a critical appraisal checklist adapted from Fowkes and Fulton (1991), using the following criteria: (a) representativeness of study sample; (b) acceptability of the control group; (c) quality of the measure of eating pathology; (d) quality of the measure of attachment or mentalization; (e) attrition; (f) blindness of assessors where this was relevant to methodology. Bias was assessed by $\mathrm{TJ}$ and $\mathrm{HC}$, with IE providing additional advice. 


\subsection{Results}

The initial search yielded a total of 460 studies, which were screened for suitability by reading the title and abstract. Of these, 111 studies were identified as potentially being suitable for inclusion, and the full texts of these studies were appraised. Following this phase, 22 studies were identified as meeting criteria, and 89 were excluded. A flowchart detailing the search and the reasons for exclusion can be found in Figure 3.1. Details of attachment studies can be found in Table 3.1, and mentalization studies in Table 3.2.

\section{$\underline{\text { 3.5.1. Risk of bias within studies }}$}

Results for the assessment of bias within individual studies are presented in Tables 3.3 and 3.4 for attachment and mentalization studies respectively. Two issues stood out as raising the risk of bias for many of the studies in this review. Firstly, the representativeness of samples was uncertain in most of the studies in the review, with most studies using non-random sampling methods. Only the studies by Le Grange et al. (2014) and Milan and Acker (2014), both of which were drawn from large population studies, used sampling methods to try to ensure a representative sample. Of the studies using clinical samples, only the studies by Zonnevylle-Bender et al. (2004) and Zonnevijlle-Bender et al. (2002) were rated as having a lower risk of bias, which was due to their use of a consecutive sampling approach. The second risk of bias came from the use of poorly validated measures, an issue which will be discussed further in the Results and Discussion sections. 
Table 3.1 Studies of attachment and eating pathology

\begin{tabular}{|c|c|c|c|c|c|}
\hline $\begin{array}{l}\text { First Author, } \\
\text { Year }\end{array}$ & Participants & $\begin{array}{l}\text { Age (Mean, } \\
\text { SD) }\end{array}$ & $\begin{array}{l}\text { Measure of } \\
\text { Attachment or } \\
\text { Mentalization }\end{array}$ & $\begin{array}{l}\text { Measure of } \\
\text { eating } \\
\text { pathology }\end{array}$ & Main results \\
\hline Cole-Detke, 1996 & $\begin{array}{l}61 \text { college women } \\
\text { Eating disorder and } \\
\text { depression }(n=19) \\
\text { Depression }(n=14) \\
\text { Eating disorders }(n=12) \\
\text { HC }(n=16)\end{array}$ & $18.6,1.0$ & $\begin{array}{l}\text { AAI Q-Sort } \\
\text { (Kobak, 1993) }\end{array}$ & $\begin{array}{l}\text { Eating Disorders } \\
\text { Inventory } \\
\text { (Garner, 1983) } \\
\text { Bulimia Criteria } \\
\text { Questionnaire } \\
\text { (Katzman, } \\
\text { 1984) }\end{array}$ & $\begin{array}{l}\text { The eating disorder group was found to have a high proportion } \\
\text { of participants in the Dismissing category }(67 \%) \text {. } \\
\text { After controlling for depression, eating disorder symptoms } \\
\text { were associated with a deactivating attachment strategy. }\end{array}$ \\
\hline Burge, 1997 & $\begin{array}{l}\text { Community sample of } \\
137 \text { female adolescents }\end{array}$ & $\begin{array}{l}18.17 \\
\text { SD not given }\end{array}$ & $\begin{array}{l}\text { Inventory of } \\
\text { Parent and Peer } \\
\text { Attachment } \\
\text { (Armsden, 1987) } \\
\text { Revised Adult } \\
\text { Attachment Scale } \\
\text { (Collins, 1990) }\end{array}$ & $\begin{array}{l}\text { Structured } \\
\text { clinical } \\
\text { interview for } \\
\text { DSM-III-R } \\
\text { (Spitzer, 1989) }\end{array}$ & $\begin{array}{l}\text { There was no main effect for attachment in predicting eating } \\
\text { pathology one year later. } \\
\text { Increases in eating pathology at time point two were found } \\
\text { when interaction terms were entered into the regression, taking } \\
\text { into account both attachment and symptomology at time point } \\
\text { one. }\end{array}$ \\
\hline Sharpe, 1998 & $\begin{array}{l}\text { Community sample of } \\
305 \text { girls }\end{array}$ & $11.9,1.3$ & $\begin{array}{l}\text { Attachment item } \\
\text { (Hazan, 1987) }\end{array}$ & $\begin{array}{l}\text { Weight } \\
\text { Concerns Scale } \\
\text { (Killen, 1994) }\end{array}$ & Insecurely attached subjects reported higher weight concerns \\
\hline Meesters, 2007 & $\begin{array}{l}\text { Non-clinical sample of } \\
405 \text { adolescents } \\
\text { (181 boys, } 224 \text { girls) aged } \\
10-16\end{array}$ & $12.5,1.5$ & $\begin{array}{l}\text { Modified } \\
\text { Relationship } \\
\text { Questionnaire } \\
\text { (Bartholomew, } \\
\text { 1991) }\end{array}$ & $\begin{array}{l}\text { Modified } \\
\text { Children's } \\
\text { Eating Attitude } \\
\text { Test (Holt, } \\
\text { 2002; Maloney, } \\
\text { 1988) }\end{array}$ & $\begin{array}{l}\text { There was a correlation between insecure attachment towards } \\
\text { mother and food preoccupation/dieting in boys only. } \\
\text { No other correlations between attachment and eating pathology } \\
\text { were found. }\end{array}$ \\
\hline
\end{tabular}


Table 3.1 Studies of attachment and eating pathology (continued)

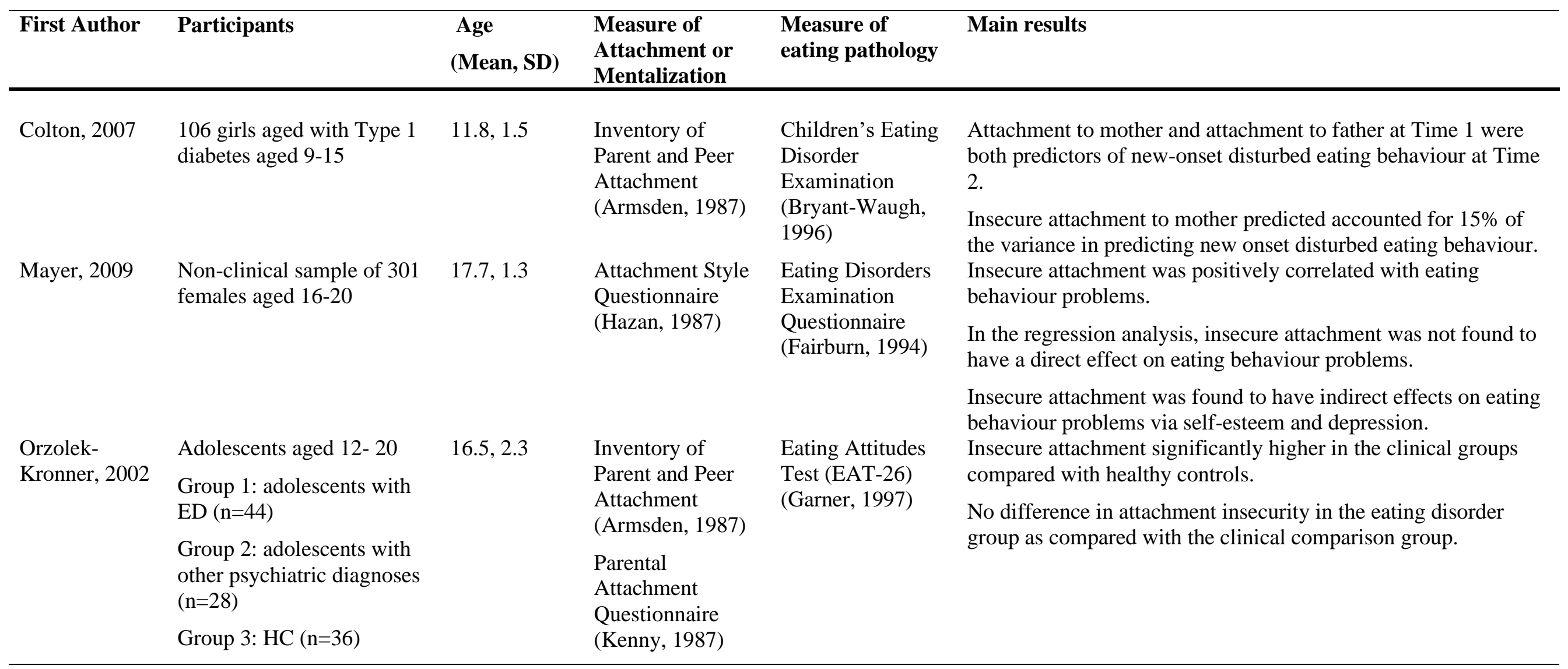


Table 3.1 Studies of attachment and eating pathology (continued)

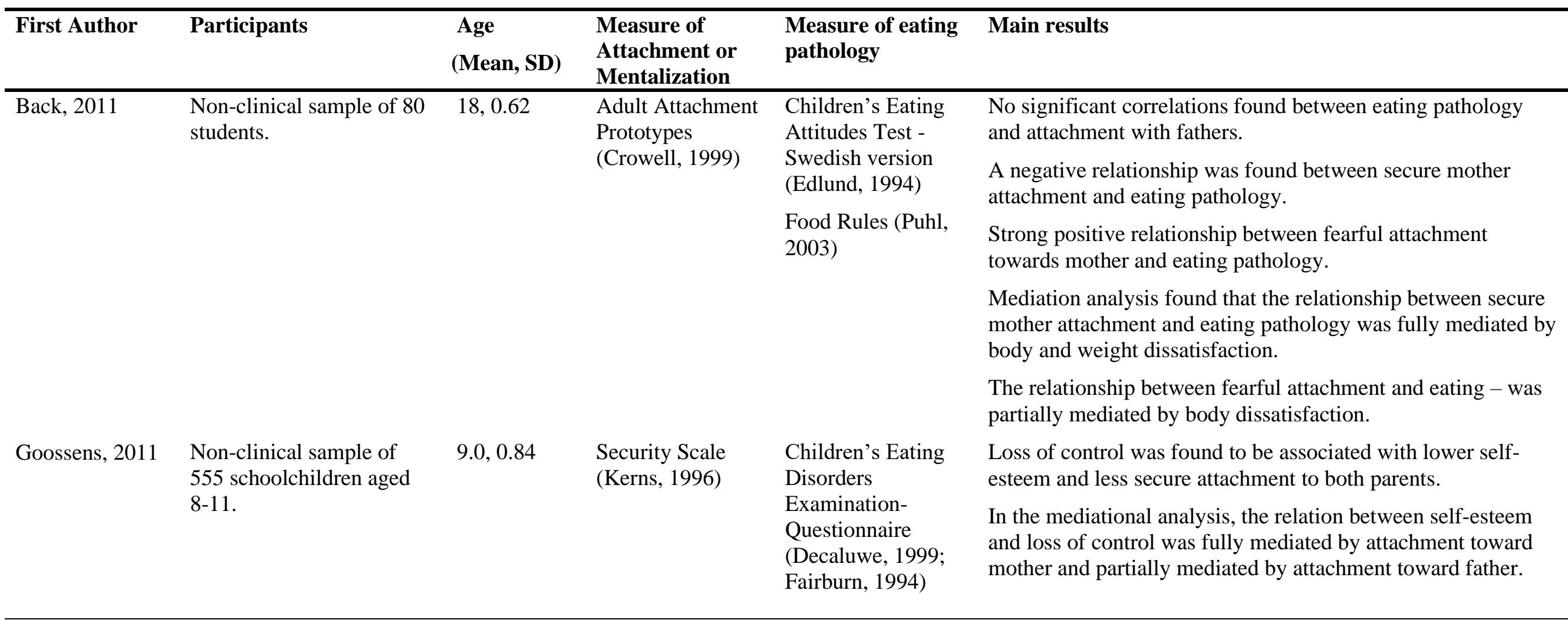


Table 3.1 Studies of attachment and eating pathology (continued)

\begin{tabular}{|c|c|c|c|c|}
\hline First Author & Participants & $\begin{array}{l}\text { Age } \\
\text { (Mean, SD) }\end{array}$ & $\begin{array}{l}\text { Measure of } \\
\text { Attachment or } \\
\text { Mentalization }\end{array}$ & $\begin{array}{l}\text { Measure of eating Main results } \\
\text { pathology }\end{array}$ \\
\hline
\end{tabular}

Goossens, 2012 Non-clinical sample of 688 children aged 8-11.

Pace, 2012

\begin{abstract}
Non-clinical sample of 233 female students aged 18-20.
\end{abstract}

$9.05,0.86$

Security Scale

(Kerns, 1996)

Children's Eating

Disorders

Examination-

Questionnaire

(Decaluwe, 1999;

Fairburn, 1994)

\section{$19.1,0.9$}

\section{Relationship \\ Questionnaire \\ (Bartholomew,}

1991)
At baseline, attachment towards mother was significantly and negatively correlated with children's eating restraint, subjective and objective binge eating, and eating, weight and shape concerns.

At baseline, attachment towards father was significantly and negatively associated with children's eating concerns, and subjective and objective binge eating.

Attachment towards mother at baseline significantly predicted increases in adjusted BMI, eating restraint and eating, weight and shape concerns one year later.

Binge Eating Scale Students with high scores on the self-report measure of binge (Gormally, 1982) eating $(n=31)$ were found to have significantly lower scores on the secure attachment scale of the attachment measure, as compared with the group with low binge-eating symptoms $(\mathrm{n}=$ 202).

The group reporting binge eating symptoms had significantly higher scores on the preoccupied and fearful scales of the attachment measure, but not on the avoidant scale. 
Table 3.1 Studies of attachment and eating pathology (continued)

\begin{tabular}{|c|c|c|c|c|c|}
\hline First Author & Participants & $\begin{array}{l}\text { Age } \\
\text { (Mean, SD) }\end{array}$ & $\begin{array}{l}\text { Measure of } \\
\text { Attachment or } \\
\text { Mentalization }\end{array}$ & $\begin{array}{l}\text { Measure of eating } \\
\text { pathology }\end{array}$ & Main results \\
\hline Cate, 2013 & $\begin{array}{l}76 \text { girls aged 9-12 were } \\
\text { screened for ED risk. } \\
\text { High risk ED group: } \mathrm{n}=15 \\
\text { Low risk ED group: } \mathrm{n}=15\end{array}$ & $\begin{array}{l}\text { High risk ED } \\
\text { group: } 10.8,0.9 \\
\text { Low risk ED } \\
\text { group: } 10.3,0.8\end{array}$ & $\begin{array}{l}\text { Inventory of } \\
\text { Parent and Peer } \\
\text { Attachment- } \\
\text { Revised (Gullone, } \\
\text { 2005) }\end{array}$ & $\begin{array}{l}\text { Children's Eating } \\
\text { Attitude Test } \\
\text { (Maloney, 1988) }\end{array}$ & $\begin{array}{l}\text { There was a significant negative correlation between } \\
\text { eating disorder risk and attachment security. }\end{array}$ \\
\hline Boone, 2013 & $\begin{array}{l}\text { Non-clinical sample of } 328 \\
\text { male and female students } \\
\text { aged } 14-20 .\end{array}$ & $17.1,1.13$ & $\begin{array}{l}\text { Experiences in } \\
\text { Close } \\
\text { Relationships } \\
\text { Scale (Fraley, } \\
\text { 2000) }\end{array}$ & $\begin{array}{l}\text { Bulimia scale of the } \\
\text { Eating Disorders } \\
\text { Inventory } 2 \\
\text { (Garner, 1991) }\end{array}$ & $\begin{array}{l}\text { Secure attachment towards mother and father was } \\
\text { negatively correlated with binge eating. } \\
\text { Anxious attachment towards mother and father was } \\
\text { associated with binge eating symptoms. } \\
\text { Avoidant attachment towards father was associated with } \\
\text { binge eating symptoms. } \\
\text { Perfectionistic self-promotion fully mediated the } \\
\text { relationship between avoidant attachment towards father } \\
\text { and binge eating. } \\
\text { Socially prescribed perfectionism fully mediated the } \\
\text { relationship between attachment avoidance towards } \\
\text { mother and binge eating. }\end{array}$ \\
\hline Le Grange, 2014 & $\begin{array}{l}\text { Non-clinical sample of } \\
1300 \text { boys and girls aged } \\
15-16 .\end{array}$ & Not reported. & $\begin{array}{l}\text { Inventory of } \\
\text { Parent and Peer } \\
\text { Attachment } \\
\text { (Armsden, 1987) }\end{array}$ & $\begin{array}{l}\text { Eating Disorders } \\
\text { Inventory } \\
\text { (Maloney, 1988) }\end{array}$ & $\begin{array}{l}\text { Higher scores for peer attachment at age } 13-14 \text {, } \\
\text { representing stronger relationships, were associated with } \\
\text { an increase in abnormal eating at age } 15-16(\mathrm{~b}=.20) \text {. } \\
\text { Parent attachment was not included in the final path } \\
\text { model. }\end{array}$ \\
\hline
\end{tabular}


Table 3.1 Studies of attachment and eating pathology (continued)

\begin{tabular}{|c|c|c|c|c|c|}
\hline First Author & Participants & $\begin{array}{l}\text { Age } \\
\text { (Mean, SD) }\end{array}$ & $\begin{array}{l}\text { Measure of } \\
\text { Attachment or } \\
\text { Mentalization }\end{array}$ & $\begin{array}{l}\text { Measure of eating } \\
\text { pathology }\end{array}$ & Main results \\
\hline \multirow[t]{3}{*}{ Milan, 2014} & \multirow[t]{3}{*}{$\begin{array}{l}\text { Non-clinical sample of } 447 \\
\text { girls. }\end{array}$} & \multirow[t]{3}{*}{$15.1,1.7$ months } & $\begin{array}{l}\text { Modified Strange } \\
\text { Situation Procedure } \\
\text { (Cassidy, 1992) }\end{array}$ & \multirow[t]{3}{*}{$\begin{array}{l}\text { Eating Attitude } \\
\text { Test (Garner, 1982) }\end{array}$} & $\begin{array}{l}\text { No evidence of a direct association between } \\
\text { attachment status in early childhood and eating } \\
\text { pathology in adolescence. }\end{array}$ \\
\hline & & & \multirow[t]{2}{*}{$\begin{array}{l}\text { Behavioural Systems } \\
\text { Questionnaire (Furman, } \\
\text { 1999) }\end{array}$} & & $\begin{array}{l}\text { At age } 15 \text {, BMI predicted disordered eating for } \\
\text { those adolescents with a history of insecure } \\
\text { attachment in early childhood. }\end{array}$ \\
\hline & & & & & $\begin{array}{l}\text { Among adolescents with a history of insecure } \\
\text { attachment in early childhood, pubertal weight gain } \\
\text { and maternal negative affect had an indirect effect } \\
\text { on eating pathology via a preoccupied relational } \\
\text { style in adolescence. }\end{array}$ \\
\hline \multirow[t]{2}{*}{$\begin{array}{l}\text { Van Durme, } \\
2015\end{array}$} & \multirow[t]{2}{*}{$\begin{array}{l}\text { Non-clinical sample of } 952 \\
\text { boys and girls aged } 10-15 .\end{array}$} & \multirow[t]{2}{*}{$12.19,1.06$} & \multirow{2}{*}{$\begin{array}{l}\text { Experiences of Close } \\
\text { Relationships - Revised } \\
\text { - Child Version } \\
\text { (Brenning, 2011) }\end{array}$} & \multirow{2}{*}{$\begin{array}{l}\text { Children's Eating } \\
\text { Disorder } \\
\text { Examination- } \\
\text { Questionnaire } \\
\text { (Bryant-Waugh, } \\
\text { 1996) }\end{array}$} & $\begin{array}{l}\text { Both attachment avoidance and anxiety had direct } \\
\text { effects on eating restraint and eating pathology. }\end{array}$ \\
\hline & & & & & $\begin{array}{l}\text { The effect of attachment avoidance and anxiety on } \\
\text { eating restraint and eating pathology was partially } \\
\text { mediated by maladaptive emotional regulation. }\end{array}$ \\
\hline
\end{tabular}

Notes: ED, Eating Disorder; HC, Healthy Controls. 
Table 3.2 Studies of mentalization and eating pathology in children and adolescents

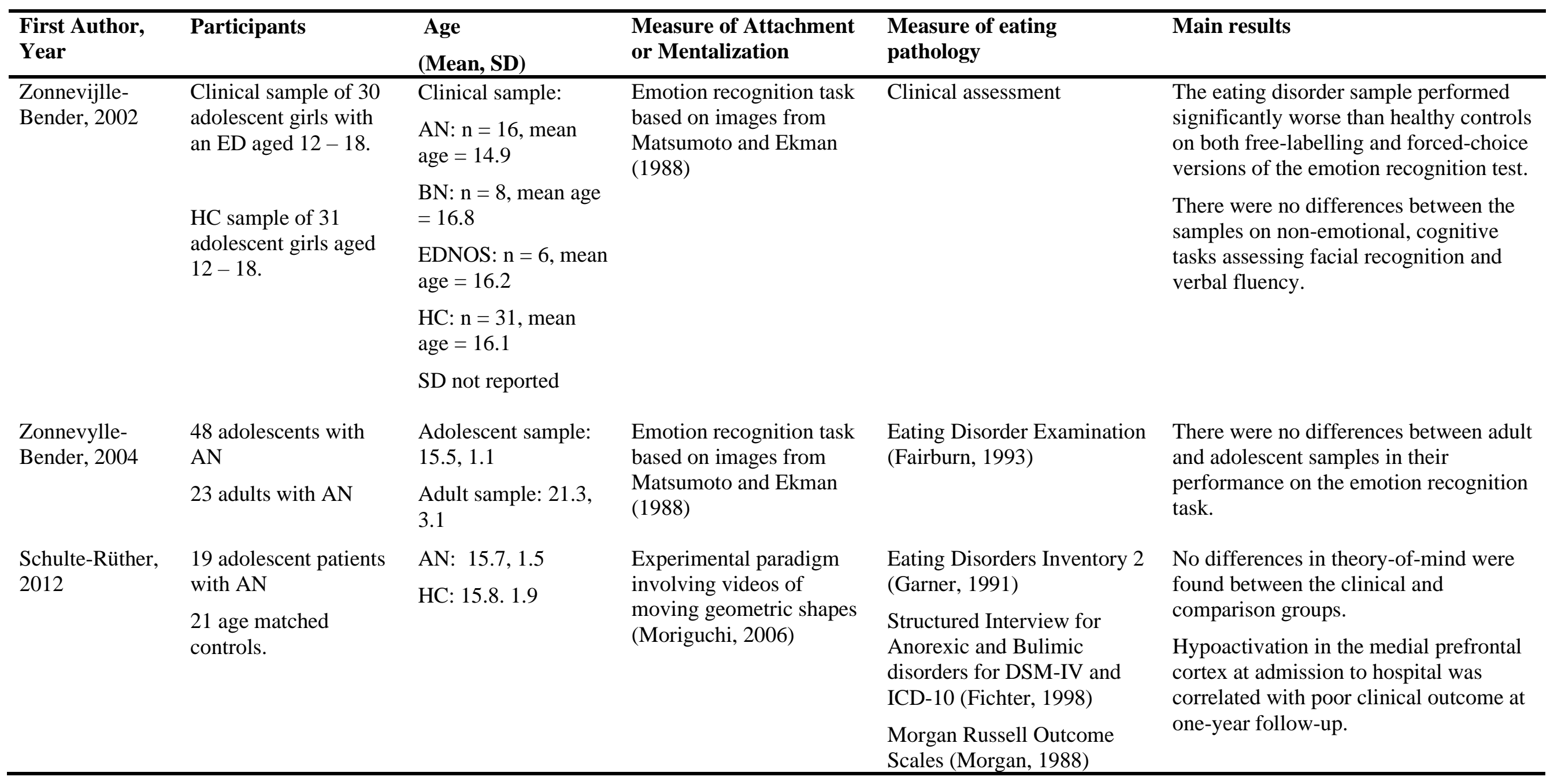


Table 3.2 Studies of mentalization and eating pathology in children and adolescents (continued)

\begin{tabular}{|c|c|c|c|c|c|}
\hline First Author & Participants & $\begin{array}{l}\text { Age } \\
\text { (Mean, SD) }\end{array}$ & $\begin{array}{l}\text { Measure of } \\
\text { Attachment or } \\
\text { Mentalization }\end{array}$ & $\begin{array}{l}\text { Measure of eating } \\
\text { pathology }\end{array}$ & Main results \\
\hline Cate, 2013 & $\begin{array}{l}76 \text { girls aged } 9-12 \\
\text { were screened for } \\
\text { ED risk. } \\
\text { High risk ED } \\
\text { group: } \mathrm{n}=15 \\
\text { Low risk ED group: } \\
\mathrm{n}=15\end{array}$ & $\begin{array}{l}\text { High risk ED } \\
\text { group: } 10.8,0.9 \\
\text { Low risk ED } \\
\text { group: } 10.3,0.8\end{array}$ & $\begin{array}{l}\text { Thematic } \\
\text { Apperception Test } \\
\text { (Murray, 1943) }\end{array}$ & $\begin{array}{l}\text { Children's Eating } \\
\text { Attitude Test } \\
\text { (Maloney, 1988) }\end{array}$ & $\begin{array}{l}\text { The group at high risk of eating disorders was found to have } \\
\text { significantly lower mentalization scores as compared with the } \\
\text { low risk group. } \\
\text { There was a significant negative correlation between eating } \\
\text { disorder risk and mentalization. }\end{array}$ \\
\hline $\begin{array}{l}\text { Rothschild- } \\
\text { Yakar, } 2013\end{array}$ & $\begin{array}{l}71 \text { female } \\
\text { inpatients and } 45 \\
\text { healthy controls. }\end{array}$ & $\begin{array}{l}\text { AN-R group: } \\
\mathrm{n}=31(15.9,1.8) \\
\text { AN-B/P group: } \\
\mathrm{n}=18(16.4,1.8) \\
\text { BN group: } \mathrm{n}=22 \\
(16.2,1.2) \\
\text { HC group: } \mathrm{n}=45 \\
(16.1,1.4)\end{array}$ & $\begin{array}{l}\text { Object } \\
\text { Representation } \\
\text { Inventory (Blatt, } \\
\text { 1993) }\end{array}$ & $\begin{array}{l}\text { Eating Disorders } \\
\text { Inventory } 2 \\
\text { (Garner, 1991) }\end{array}$ & $\begin{array}{l}\text { Patients with eating disorders presented with a significantly } \\
\text { lower level of symbolic representation and with more } \\
\text { malevolent representations of their parents in comparison to } \\
\text { the controls. } \\
\text { Across all study participants, a more benevolent parental } \\
\text { representation, specifically with father, combined with better } \\
\text { mentalization abilities, was found to indirectly predict lower } \\
\text { eating disorder symptoms, via the reduction of distress. }\end{array}$ \\
\hline Lulé, 2014 & $\begin{array}{l}\text { Clinical sample of } \\
15 \text { adolescent } \\
\text { females with AN. }\end{array}$ & $\begin{array}{l}\text { AN: } 16.2,1.26 \\
\text { HC: } 16.5,1.09\end{array}$ & $\begin{array}{l}\text { Emotion recognition } \\
\text { task based on images } \\
\text { from Matsumoto and } \\
\text { Ekman (1988) }\end{array}$ & $\begin{array}{l}\text { Eating Disorders } \\
\text { Examination } \\
\text { Questionnaire } \\
\text { (Fairburn, 1994) }\end{array}$ & $\begin{array}{l}\text { Adolescents with anorexia nervosa showed a shorter reaction } \\
\text { time for recognition of emotions relative to controls. } \\
\text { Adolescents with anorexia nervosa showed a tendency to } \\
\text { recognise disgust less correctly than controls. }\end{array}$ \\
\hline & $\begin{array}{l}\text { HC sample of } 15 \\
\text { adolescent females. }\end{array}$ & & & $\begin{array}{l}\text { Eating Disorders } \\
\text { Inventory } 2 \\
\text { (Garner, 1991) }\end{array}$ & $\begin{array}{l}\text { Perception of the basic emotions fear, surprise, sadness and } \\
\text { anger did not differ significantly between groups. } \\
\text { Adolescents with anorexia nervosa showed increased ability to } \\
\text { recognize happiness relative to the control group. }\end{array}$ \\
\hline
\end{tabular}


Table 3.2 Studies of mentalization and eating pathology in children and adolescents (continued)

\begin{tabular}{|c|c|c|c|c|c|}
\hline First Author & Participants & $\begin{array}{l}\text { Age } \\
\text { (Mean, SD) }\end{array}$ & $\begin{array}{l}\text { Measure of } \\
\text { Attachment or } \\
\text { Mentalization }\end{array}$ & $\begin{array}{l}\text { Measure of eating } \\
\text { pathology }\end{array}$ & Main results \\
\hline Lang, 2015 & $\begin{array}{l}61 \text { adults and } 36 \\
\text { adolescents with } \\
\text { AN aged 11-47 } \\
\text { HC sample of } 69 \\
\text { adults and } 27 \\
\text { adolescents aged } \\
11-53 \text {. }\end{array}$ & $\begin{array}{l}\text { Adolescent AN: } \\
15.08,1.70 \\
\text { Adolescent HC: } \\
14.56,1.91\end{array}$ & $\begin{array}{l}\text { Point-light walker task } \\
\text { (Atkinson, 2007) }\end{array}$ & $\begin{array}{l}\text { Eating Disorders } \\
\text { Examination } \\
\text { Questionnaire } \\
\text { (Fairburn, 1994) } \\
\text { Structured clinical } \\
\text { interview for DSM- } \\
\text { IV-TR Axis 1 } \\
\text { Disorders (First, 2002) }\end{array}$ & $\begin{array}{l}\text { Adolescents with anorexia nervosa were less } \\
\text { accurate than adolescent healthy controls in } \\
\text { recognising sadness. } \\
\text { Both adolescents and adults with anorexia nervosa } \\
\text { were less accurate than the healthy control group in } \\
\text { recognising sadness. However, within the clinical } \\
\text { sample, adolescents were poorer than adults in } \\
\text { recognising sadness. } \\
\text { There were no other differences in emotion } \\
\text { recognition between clinical and healthy control } \\
\text { samples. }\end{array}$ \\
\hline
\end{tabular}

Notes: ED, Eating Disorder; AN, Anorexia Nervosa; BN, Bulimia Nervosa; EDNOS, Eating Disorder Not Otherwise Specified; AN-R, Anorexia Nervosa - Restrictive Type; AN-B/P, Anorexia Nervosa Binge/Purge Type; HC, Healthy Control 
Figure 3.1 Flowchart for search strategy

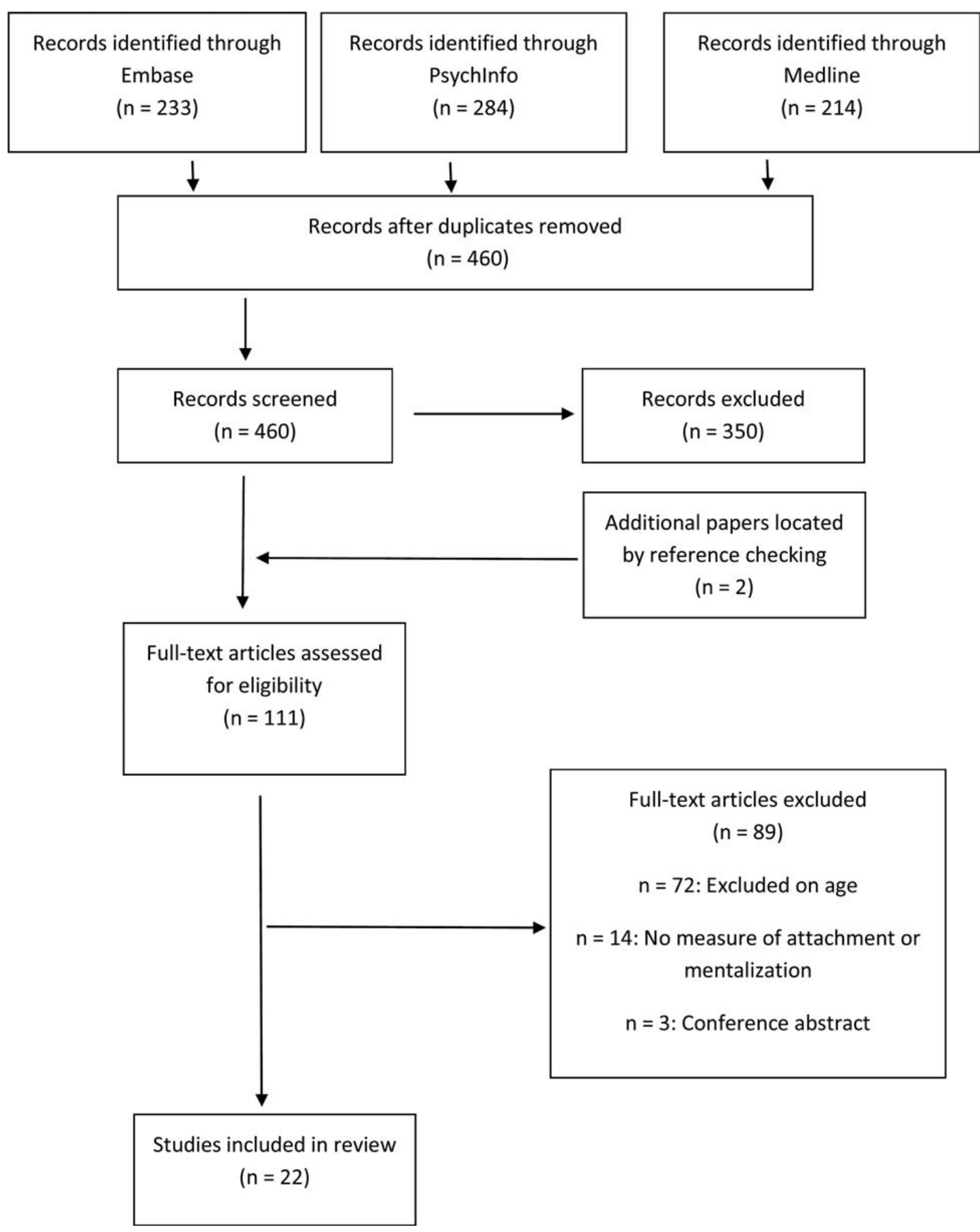


Table 3.3 Assessment of risk of bias within individual studies of attachment and eating pathology

\begin{tabular}{lcllccccc}
\hline First author & Date & $\begin{array}{l}\text { Study } \\
\text { type }\end{array}$ & Sampling & $\begin{array}{l}\text { Control } \\
\text { Group }\end{array}$ & $\begin{array}{l}\text { Measure } \\
\text { of eating } \\
\text { pathology }\end{array}$ & $\begin{array}{l}\text { Measure of } \\
\text { attachment }\end{array}$ & Attrition & Blindness \\
\hline Cole-Detke & 1996 & $\mathrm{C}$ & + & + & - & - & & \\
Burge & 1997 & $\mathrm{P}$ & + & & - & - & - & \\
Sharpe & 1998 & $\mathrm{C}$ & + & & - & + & & \\
Orzolek- & 2002 & $\mathrm{C}$ & + & - & + & - & & \\
Kronner & & & + & & & & & \\
Meesters & 2007 & $\mathrm{C}$ & + & & - & + & & \\
Colton & 2007 & $\mathrm{P}$ & + & & - & - & - & \\
Mayer & 2009 & $\mathrm{C}$ & + & & - & - & & \\
Back & 2011 & $\mathrm{C}$ & + & & - & - & & \\
Goossens & 2011 & $\mathrm{C}$ & + & & - & - & \\
Goossens & 2012 & $\mathrm{P}$ & + & & - & - & + & \\
Pace & 2012 & $\mathrm{C}$ & + & & - & - & & \\
Boone & 2013 & $\mathrm{C}$ & + & & - & - & & \\
Cate & 2013 & $\mathrm{C}$ & + & + & - & - & & \\
Le Grange & 2014 & $\mathrm{P}$ & - & & - & + & - & \\
Milan & 2014 & $\mathrm{P}$ & - & & - & - & - & \\
Van Durme & 2015 & $\mathrm{C}$ & + & & - & - & & \\
\hline
\end{tabular}

Notes: C, cross-sectional; P, prospective; E, experimental; +, higher risk of bias; -, lower risk of bias; blank, not applicable 
Table 3.4 Assessment of risk of bias within individual studies of attachment and eating pathology

\begin{tabular}{|c|c|c|c|c|c|c|c|c|}
\hline First author & Date & $\begin{array}{l}\text { Study } \\
\text { type }\end{array}$ & Sampling & $\begin{array}{l}\text { Control } \\
\text { Group }\end{array}$ & $\begin{array}{l}\text { Measure } \\
\text { of eating } \\
\text { pathology }\end{array}$ & $\begin{array}{l}\text { Measure of } \\
\text { mentalization }\end{array}$ & Attrition & Blindness \\
\hline $\begin{array}{l}\text { Zonnevylle- } \\
\text { Bender }\end{array}$ & 2002 & $\mathrm{C}$ & - & - & + & - & & \\
\hline $\begin{array}{l}\text { Zonnevijlle - } \\
\text { Bender }\end{array}$ & 2004 & $\mathrm{C}$ & - & & - & - & & \\
\hline $\begin{array}{l}\text { Schulte- } \\
\text { Ruther }\end{array}$ & 2012 & $\mathrm{P}$ & - & - & - & - & - & \\
\hline $\begin{array}{l}\text { Rothschild- } \\
\text { Yakar }\end{array}$ & 2013 & $\mathrm{C}$ & + & - & - & - & & - \\
\hline Cate & 2013 & $\mathrm{C}$ & + & + & - & + & & - \\
\hline Lule & 2014 & $\mathrm{C}$ & + & - & - & - & & \\
\hline Lang & 2015 & $\mathrm{C}$ & + & - & - & + & & \\
\hline
\end{tabular}

Notes: C, cross-sectional; P, prospective; E, experimental; +, higher risk of bias; -, lower risk of bias; blank, not applicable 


\subsubsection{Cross-sectional studies of attachment and eating pathology}

Eleven studies used cross-sectional designs to investigate associations between eating pathology and attachment in children and adolescents. In all eleven studies, an association between these two variables was reported.

Three studies investigated attachment and eating pathology in community samples of pre-adolescent or early adolescent children. In Sharpe et al.'s (1998) study of 305 girls, participants who were classed as insecurely attached reported significantly higher weight concerns. However, attachment was assessed using Hazan \& Shaver's (1987) attachment item, in which participants are asked to choose which out of three short paragraphs best described their feelings. The paragraphs are based on adult romantic attachment styles, and it is unclear whether this is a valid measure of attachment in pre-adolescent children. A similar problem applies to the study by Meesters et al. (2007), in which 405 children aged 10-16 were given a modified version of the Relationship Questionnaire (Bartholomew \& Horowitz, 1991), which is another measure of adult romantic attachment styles. The modified measure aims to assess attachment to mother, father and peers with one item for each attachment figure. The validity and reliability of the adapted children's version of the instrument is low. The study found a correlation between insecure attachment towards mother and food preoccupation/dieting in boys only.

Cate et al. (2013) used the Inventory of Parent and Peer Attachment (IPPA) in their study (Armsden \& Greenberg, 1987). In a sample of 76 girls, a significant negative correlation between eating pathology and attachment security was found, when the latter was measured on a single dimension of security. The IPPA was also used by 
Orzolek-Kronner (2002), who investigated attachment and eating pathology within three samples aged 12-20 - a sample with eating disorders, a clinical comparison group with other psychiatric diagnoses and a healthy control sample. Scores for attachment security were similar in the eating disorder and clinical comparison groups, but were significantly lower in the control group. This suggests that there is an association between attachment insecurity and psychiatric morbidity that is not specific to eating pathology.

Three studies investigated associations between attachment and eating pathology in late-adolescents. Pace et al. (2012) examined binge eating symptoms and attachment in a community sample of 233 late adolescent students. The group of students with high self-reported binge eating symptoms was found to have significantly lower scores on the secure attachment scale of the Relationship Questionnaire (Bartholomew \& Horowitz, 1991). On the insecure attachment sub-scales, the group reporting binge eating symptoms had significantly higher scores on the preoccupied and fearful subscales of the attachment measure, but not on the avoidant subscale.

Cole-Detke and Kobak (1996) investigated both eating disorder and depressive symptoms in a sample of college women. Based on a three-way coding of attachment classification derived from the AAI Q-Sort (Kobak et al., 1993), the group with high self-reported eating pathology was found to have a high proportion of participants in the Dismissing category (67\%). Once depressive tendencies were controlled for in the analysis, eating disorder symptoms were associated with deactivating attachment strategies, such as attempting to divert attention away from attachment cues in the interview. 
Mayer et al. (2009) investigated the direct and indirect effects of risk factors for eating behavior problems in a non-clinical sample of late adolescent females. In keeping with other studies, insecure attachment was positively correlated with eating behavior problems. However, in the regression analysis, insecure attachment was not found to have a direct effect on eating behavior problems, but to have indirect effects via selfesteem and depression. This raises the possibility of self-esteem and depression as potentially important mediators of the relationship between attachment and eating pathology.

\section{$\underline{\text { 3.5.3 Cross-sectional studies involving tests of mediation }}$}

Three cross-sectional studies testing mediation models were identified.

Back (2011) examined eating behaviors, attachment and parental upbringing in a sample of 80 high school students. The negative relationship between secure mother attachment and eating pathology was fully mediated by body and weight dissatisfaction, whereas the positive relationship between fearful attachment and eating was only partially mediated by body dissatisfaction.

Van Durme et al. (2015) investigated the role of emotional regulation as a potential mediator of the relationship between insecure attachment and eating pathology in a non-clinical sample of 952 children aged 10-15. Maladaptive emotional regulation was found to partially mediate the effect of both attachment avoidance and anxiety on eating restraint and eating pathology. A limitation of the study is the use of the Experiences in Close Relationships Scale-Revised (Brenning et al., 2011), which has poor evidence of construct validity (Brenning et al., 2011). 
Boone (2013) examined the association between attachment and binge eating symptoms in a community sample of 328 students aged 14-20. Perfectionistic selfpromotion was found to fully mediate the relationship between avoidant attachment towards father and binge eating. Socially prescribed perfectionism fully mediated the relationship between attachment avoidance towards mother and binge eating.

Finally, attachment itself was investigated as a mediator of the relationship between self-esteem and loss of control of eating in a study by Goossens et al. (2011), in a community sample of 555 pre-pubertal children aged 8-11 years. The relationship between self-esteem and loss of control was fully mediated by attachment toward mother and partially mediated by attachment toward father. A limitation of all four studies is that, by the nature of their cross-sectional design, they provide evidence of associations. Further testing within longitudinal study designs is needed to confirm the hypothesized mediating role of these variables.

\subsubsection{Prospective studies of attachment and eating pathology}

Five prospective studies were identified in this review.

Milan and Acker (2014) investigated the effect of attachment insecurity in early infancy on responsivity to risk factors for eating disorders in a sample of 447 girls aged 15. Attachment was assessed at 36 months, using Cassidy et al.'s (1992) modified Strange Situation. Eating attitudes were assessed at age 15 using the Eating Attitudes Test (Garner et al., 1982). Maternal affect was measured at ages 11, 12 and 15, and averaged across time-points in the mediation analysis. At age 15 the study also 
recorded the child's relational style, body mass index (BMI) and pubertal weight change, as compared with the BMI recorded at age 9. The study found no evidence of a direct association between attachment status in early childhood and eating pathology in adolescence. However, BMI predicted disordered eating for those adolescents with a history of insecure attachment in early childhood. Among adolescents with a history of insecure attachment in early childhood, pubertal weight gain and maternal negative affect had an indirect effect on eating pathology via a preoccupied relational style in adolescence.

Goossens et al. (2012) investigated attachment as a predictor of weight gain and eating pathology in a community sample of 688 preadolescents. Attachment was assessed using Kerns et al.'s (1996) Security Scale, which yields an overall score of security. Eating pathology was assessed using a Dutch adaptation of the Children's Eating Disorder Examination Questionnaire (ChEDE-Q) (Decaluwe \& Braet, 1999). Measurement of variables was repeated one year later. At baseline, attachment to mother and attachment to father were both negatively correlated with eating pathology, but the magnitude of the correlation was greater for maternal attachment. Attachment toward mother at baseline significantly predicted increases in adjusted BMI, eating restraint and eating, weight and shape concerns one year later.

Le Grange et al. (2014) report a study investigating infant and child pathways to abnormal eating attitudes in adolescence, drawing on a large Australian cohort of children. Eleven waves of data were collected from infancy to age 15-16. At age 1314, participants completed the IPPA (Armsden \& Greenberg, 1987). A path analysis 
was performed to identify developmental correlates of abnormal eating as measured at age 15-16. Peer attachment, but not parent attachment, was included in the final path model. Surprisingly, higher scores for peer attachment, representing stronger relationships, were associated with an increase in abnormal eating at age 15-16. Correlations between parent and peer attachment and eating pathology are not reported.

Two further longitudinal studies met criteria for review, both of which are limited by small sample sizes. Colton et al. (2007) investigated attachment and eating pathology in a sample of 106 girls with Type 1 diabetes. Attachment security to mother and father, as rated on the IPPA (Armsden \& Greenberg, 1987), were both predictors of new-onset disturbed eating behaviour one year later. However, only 10 girls in the sample reported new disturbed eating behaviour at the second time point, and external validity is compromised by the specificity of the sample. Burge et al. (1997) conducted a study of attachment and psychopathology in a community sample of 137 late adolescent women. Attachment was assessed by self-report measures, but psychopathology was assessed via structured interview. The study found no main effect for attachment on eating pathology one year later. However, when interaction terms were entered into the regression analysis, the interaction of attachment with initial symptomology was predictive of increases in eating pathology. However, only two women in the sample met criteria for eating disorders, with a further five women exhibiting eating disorder symptomology. Overall, the longitudinal studies in this review have a number of limitations. Firstly, the relationship between self-report measures of eating pathology and caseness is problematic (Williams et al., 1982), in part because eating disorders meeting diagnostic threshold are relatively uncommon. 
The EAT questionnaire (Garner et al., 1982), which was used in Milan and Acker's (2014) study, has been found to have high rates of false positives and false negatives (1992), and Eisler and Szmukler (1985) found that social class was a confounding variable in their community sample of British schoolgirls who completed the EAT. Similar doubts apply to the ChEDE-Q (Decaluwe \& Braet, 1999) used in the Goossens et al. (2012) study. The ChEDE-Q was validated in a sample of obese youngsters and was found to produce significantly different results as compared with interview measures in respect of binge eating symptoms (Decaluwé \& Braet, 2004). Indeed, agreement levels between the ChEDE-Q and interview were lower than those reported for the adult self-report EDE-Q (Fairburn \& Beglin, 1994) on which it is based, and its developers suggest that further validation work is necessary, using samples with a broader range of eating disorders. The EDI (Garner et al., 1983) used in Le Grange et al.'s (2014) study, is also not a diagnostic tool (Pike, 2005). The one longitudinal study which used a diagnostic interview to assess eating pathology, by Burge et al. (1997), did not find attachment to predict increases in eating pathology when controlling for previous eating pathology. Thus the findings of the longitudinal studies suggesting that attachment insecurity predicts later eating pathology should be interpreted with caution, given the uncertain relationship between high scores on self-report measures of eating pathology and clinically significant eating disorder symptoms. This limitation also applies to all the other studies in this review using self-report measures of eating pathology in community samples.

A further limitation of the Goossens et al. (2012) study is the confound between eating pathology and weight gain. The authors take categorical BMI increases (e.g. from overweight to obese) to be indicative of eating pathology, yet they do not differentiate 
between weight gain of those who are underweight and those who are normal or overweight. In Milan and Acker's (2014) study, pubertal weight change measured between the ages of 9 and 15 was found to indirectly predict increased disordered eating attitudes, although only in the sample with an insecure attachment history at 36 months. Pubertal weight change is reported without reference to BMI categories, such as overweight. A fair reading of Milan and Acker's (2014) findings is that insecure attachment may have a moderating effect on the impact of weight gain around puberty on eating attitudes, albeit the effect is modest. As acknowledged by the authors, it is not clear to what extent such a finding applies to clinical samples with eating disorders.

The study by Le Grange et al. (2014) is notable in being the only study in this review that did not report an association between parent attachment and eating pathology. Also of interest is the finding that stronger peer attachment was associated with increases in eating pathology. However, the conclusion validity of this study is threatened by the fact that the attachment measure used was significantly shortened.

\section{$\underline{\text { 3.5.5 Studies of mentalization and eating pathology }}$}

Seven studies of mentalization and eating pathology were identified.

\section{$\underline{\text { 3.5.6 Studies investigating emotion recognition }}$}

Four studies were identified which investigated emotion recognition abilities in adolescents with eating disorders. Three of these studies (Lulé et al., 2014; Zonnevijlle-Bender et al., 2002; Zonnevylle-Bender et al., 2004) used images of facial expressions developed by Matsumoto and Ekman (1988). These are a well-validated 
set of images to assess facial recognition, with several studies supporting their validity and reliability (Biehl et al., 1997).

Zonnevijlle-Bender et al. (2002) investigated emotion recognition in a clinical sample of 30 adolescent girls with an eating disorder aged $12-18$, and compared this with a healthy control sample. Participants were shown images of female models showing one of seven emotions (happiness, anger, sadness, fear, disgust, surprise and contempt). The eating disorder sample performed significantly worse than healthy controls in recognizing emotions. This procedure was repeated in a second study (Zonnevylle-Bender et al., 2004) by this research group, this time comparing the performance of adolescent and adult samples with AN. The authors found no significant difference between the two groups in their ability to recognize emotions.

Lulé et al. (2014) investigated accuracy and speed of emotion labelling in a sample of 15 adolescent females with $\mathrm{AN}$, as compared to 15 age and sex-matched healthy controls. Participants were shown images of faces displaying the six basic emotions (anger, fear, sadness, surprise, disgust and happiness) on a computer. Adolescents with AN showed a shorter reaction time for recognition of emotions relative to controls. The accuracy of perception did not differ between the clinical and control groups for fear, surprise, sadness and anger. Adolescents with AN showed less accuracy in recognizing disgust, but greater accuracy in recognizing happiness. This study has a risk of bias due to the small sample size. Moreover, the clinical sample included both inpatients and outpatients, whose treatment ranged from 8 to 736 days. Data on how many of the clinical sample had been admitted to hospital are not provided. It is 
therefore hard to assess the representativeness of the clinical sample. A strength of the study is that the clinical sample were physically stable, thus implying that their performance was not compromised by effects of starvation.

Lang et al. (2015) investigated emotion recognition in adolescents and adults with AN using a body motion paradigm, as compared with health controls. Participants viewed video clips of actors walking from left to right whilst portraying one of four emotions (anger, fear, happiness or sadness) or an emotionally neutral state. The actors were filmed in the dark and had small lights attached to various body parts, so that the stimuli appeared to participants as white dots on a dark background. The study found no differences between the clinical and healthy control samples with respect to recognizing emotions, with the exception of sadness. Adolescents with AN were less accurate than adolescent healthy controls in recognizing sadness. Both adolescents and adults with AN were less accurate than the healthy control group in recognizing sadness. However, within the clinical sample, adolescents were poorer than adults in recognizing sadness.

One limitation of the study concerns the validity and reliability of the emotion recognition task. The authors developed the scoring method using a small reference group of 15 healthy controls. This reference group did not view the clips portraying 'neutral' emotions, which could have introduced bias, as respondents may give different answers regarding emotion when the stimuli is changed, as the sequence and content of the materials may influence interpretation. 


\subsubsection{Other studies of mentalization and eating pathology}

Three other studies were identified which used disparate study designs and measures to investigate mentalization and eating pathology.

In the study by Cate et al. (2013), which examined both mentalization and attachment, 76 girls (aged 9-12) were administered self-report measures of eating pathology. Subjects scoring at the highest and lowest extremes of eating pathology were administered a picture task developed by Murray (1943) in which they had to make up a story relating to each of the five pictures. Mentalizing capacity was assessed using a coding system applied to the transcripts of the children's responses (Symons et al., 2005). The total mentalization score was based on the girls' use of mentalistic language. The group which scored highly on the measure of eating pathology was found to have significantly lower mentalization scores compared with the group with low scores. Significant negative correlations were found between eating disorder risk and attachment styles, and between eating disorder risk and mentalization.

Rothschild-Yakar et al. (2013) examined mentalizing in a sample of female inpatients with eating disorders. The clinical sample comprised 71 female inpatients aged 14-19 and an age-matched sample of healthy controls. Mentalizing in self and others was assessed using the Object Representation Inventory (ORI) (Blatt et al., 1993). Patients with eating disorders presented with a significantly lower level of symbolic representation and with more malevolent representations of their parents in comparison to controls. Across all study participants, a more benevolent parental representation, specifically with father, combined with better mentalization abilities, 
was found to indirectly predict lower eating disorder symptoms, via the reduction of distress.

Schulte-Rüther et al. (2012) conducted a study of 19 adolescent patients (aged 12-18) with AN and 21 age-matched controls. Participants were investigated using functional magnetic resonance imaging during TOM tasks at two time-points - for the patients with anorexia this was at admission and discharge from hospital. TOM was assessed using an experimental paradigm involving 15 -second videos of three white geometric shapes moving against a black background. Participants were asked whether the shapes were 'friends' or not based on the contingency of the interactions, which were designed to be interpreted as social. No differences were found between the clinical and comparison groups. However, irrespective of time-point, patients with AN showed reduced activation in the middle and anterior temporal cortex and in the medial prefrontal cortex. Hypoactivation in the medial prefrontal cortex at admission to hospital was correlated with poor clinical outcome at 1-year follow-up. The medial prefrontal cortex is known to be a region of the brain that is activated during mentalizing tasks (Blakemore, 2008; Frith \& Frith, 2003).

In all three studies, the external validity of the findings is compromised by the relatively poor validity of the instruments used to assess mentalization. In Cate et al.'s (2013) study, the use of Symons et al.'s (2005) system to code responses to Murray's (1943) Thematic Apperception Test is a novel but not yet validated test of mentalization in 9-12 year olds. No other measure of mentalization was used, thus the concurrent validity of this measurement approach is unknown. Similarly, the use of 
Blatt et al.'s (1993) ORI also lacks validity data in adolescent samples. Finally, behavioral performance on the experimental measure used in Schulte-Rüther et al.'s (2012) study did not discriminate between AN and non-clinical samples in either this study, or in the study by McAdams and Krawczyk (2011), although both studies reported differences at the level of neural function. As such, this measure has been rated as presenting a lower risk of bias, although it should be noted that the sample sizes in these brain imaging studies were small.

\subsection{Discussion}

This review has found limited evidence of an association between mentalizing difficulties and eating pathology in children and adolescents. Thus far, the most replicated finding is that of emotion recognition difficulties in adolescent samples with eating disorders, particularly AN, although the heterogeneity of the samples in this sub-group of studies reduces the strength of this finding. All the studies in this review reported an association between mentalizing difficulties and eating pathology, and an association was found between insecure attachment and mentalizing difficulties in the one study which examined the two variables (Cate et al., 2013). However, caution is warranted in interpreting findings given that small sample sizes and poorly-validated measures of mentalization were a limitation of several studies. A gap in the adolescent literature is the absence of studies comparing mentalizing ability in clinical samples of young people with anorexia nervosa whilst ill and following recovery. The evidence in this review thus does not speak to the issue of mentalizing difficulties as a trait or state. Within the taxonomy developed by Kraemer et al. (1997), current evidence suggests that mentalizing difficulties may be a correlate of eating pathology in children and adolescents. 
With regard to attachment, a positive correlation between attachment insecurity and eating pathology in childhood and adolescence was found in 14 of the 15 studies in this review. The validity of this association is strengthened by the use of developmentally appropriate, well-validated measures of attachment, such as Kerns et al.'s (1996) Security Scale, within large samples, such as in the study by Goossens et al. (2012). However, there is a risk that the association between attachment and eating pathology may be confounded by variables such as low self-esteem and depression, and future studies in this area need to look more closely at potential confounders which might impact on appraisals of self and others.

The evidence of an association between attachment insecurity and eating pathology replicates the findings of the adult literature (Kuipers \& Bekker, 2012; Tasca \& Balfour, 2014). Of note, this association has been found in non-clinical samples across the age range from pre-adolescence (Cate et al., 2013; Goossens et al., 2011, 2012) to late adolescence (Bäck, 2011; Cole-Detke \& Kobak, 1996). The studies in this review also found an association between insecure attachment and a broad range of eating pathology (Goossens et al., 2012; Van Durme et al., 2015).

In terms of the question of etiology, the findings of the longitudinal studies in this review merit particular attention. Milan and Acker (2014) found that attachment insecurity in infancy is a weak predictor of later eating pathology, whereas Goossens et al. (2012) found that attachment insecurity in the pre-adolescent period predicted the development of eating pathology one year later much more robustly. Le Grange et 
al. (2014) found attachment to peers, but not parents, predicted eating pathology in mid-adolescence. This latter study has the lowest reliability of the three longitudinal studies in this review, on account of its use of a significantly shortened version of its attachment measure. The Milan and Acker (2014) study further discredits what O'Kearney (1996) has referred to as unitary causation models - quite clearly, early attachment insecurity is not the cause of eating disorders. The contrast between the findings of Milan and Acker (2014) and Goossens et al. (2012) also emphasizes the point that insecure attachment as assessed in adolescence or adulthood cannot be taken to be evidence for insecure attachment in infancy. The stability of attachment from infancy to adulthood is limited (Pinquart et al., 2013) and Fearon et al. (2014) recently found evidence for a strong genetic influence on attachment representations in adolescence. These findings help make sense of why the predictive value of insecure attachment appears to be stronger in the pre-adolescent phase as compared with infancy. The available evidence suggests that insecure attachment is both a correlate and risk factor for eating pathology. However, given that no study has demonstrated that change in attachment alters the risk of eating pathology, insecure attachment has not been established as a causal risk factor.

\subsubsection{Implications for theory}

The findings of this review, and other developments in the fields of attachment and mentalization, suggest a need to revise theory regarding the relationships between attachment, mentalization and eating pathology. Firstly, infant attachment has been considered along with other perinatal factors, such as obstetric difficulties, in developmental models of risk for anorexia nervosa (Connan et al., 2003; WoerwagMehta \& Treasure, 2008). However, the prospective studies examined in this review 
(Goossens et al., 2011, 2012; Milan \& Acker, 2014) suggest that it is the pre-pubertal and pubertal phases of development in which attachment may come to play a more important role in the development of eating pathology.

Secondly, the meaning of adolescent attachment needs to be reconsidered as more than a marker of parent-child relationships, since it appears to be partly heritable (Fearon et al., 2014) and more closely associated with qualities of peer relationships than with parent-child relationships (Allen \& Miga, 2010). Theory needs to include a role for peer attachment in the development of eating pathology, as suggested by the findings of Le Grange et al.'s (2014) study. The potential role of affect regulation needs expanding in theoretical models, as supported by findings from both the child (Van Durme et al., 2015) and adult (Tasca et al., 2009) literature. Moreover, it has been suggested that adolescent attachment interviews are better viewed as measuring affect regulation in the context of social interaction, rather than measuring parent-child relationships per se (Allen \& Miga, 2010).

Thirdly, theoretical links between attachment and mentalization need development. Mentalizing ability is a dynamic capacity that is influenced by stress and arousal, particularly in the context of specific attachment relationships (Allen et al., 2008). Under conditions of high arousal, patterns of brain activity 'switch' from flexibility to automaticity, resulting in the loss of mentalizing (Fonagy et al., 2012). The threshold for this 'switch' to automatic, non-mentalizing modes of thought varies between individuals. However, the ability to tolerate strong negative affects is a marker for secure attachment (Fonagy et al., 2012). Individuals with insecure attachment 
representations are thus more likely to experience mentalizing failures in the context of affective arousal. This could have implications for treatment, particularly when using eating disorders focused family therapy (FT-AN) - sometimes referred to as family based treatment or FBT - where an early focus is on parents helping the young person to eat (Eisler, Le Grange \& Lock, 2015; Eisler, Wallis \& Dodge, 2015). If adolescents and/or other family members become highly emotionally aroused during therapy sessions, they may be more likely to interpret material arising from the session in negative terms and feel criticised or blamed. This could potentially reduce family cohesion and damage the therapeutic alliance, thereby moderating the effectiveness of treatment. This may be a particular risk for adolescents and adults categorised as having preoccupied attachments on interview measures of attachment (George et al., 1985; Shmueli-Goetz et al., 2008). Individuals assigned this category display on-going anger towards attachment figures, often seeming 'caught up' in past grievances. It is possible that adolescents displaying attachment preoccupation may benefit less from FT-AN, since they may experience their parents' attempts to help them manage their eating as controlling or even punitive. By contrast, adolescents with secure attachment representations may be more likely to experience parental attempts to help them manage their eating as an act of care and support.

\subsubsection{Implications for research}

Stice et al. (2012) have called for methodologically rigorous prospective risk factor studies that include a broad range of variables to investigate the possibility of interaction between risk factors. On the basis of this review, attachment, mentalization and affect regulation should be included as variables within such studies. Other known risk factors for eating disorders, such as genetic vulnerability and perfectionism (Egan 
et al., 2011; Polivy \& Herman, 2002) will also need inclusion, as should potential mediators such as depression and self-esteem. Given the relative rarity of clinical cases of eating disorders, prospective studies of at-risk community samples, drawn from large population studies, will be needed to further understanding regarding the interaction of risk factors. Case-control study designs, in which participants are matched for level of eating pathology, but differ on attachment and mentalization, could help to shed light on whether, and how, these variables are implicated in the development of eating pathology for some individuals.

With regard to measurement of attachment and mentalization, both are complex constructs and it will be necessary to use a range of measures to investigate them. It is known that self-report and interview measures tap different aspects of attachment (Roisman et al., 2007). Studies of adolescent eating disorder samples using interviewbased measures such as the Child Attachment Interview (Shmueli-Goetz et al., 2008) would help to elucidate aspects of attachment representations that are not available to conscious appraisal, such as idealisation of attachment figures (Venta et al., 2014). Similarly, mentalizing is also a multi-faceted concept and requires a range of assessment measures to explore fully. Importantly, well-validated measures of mentalization for children and adolescents have now been developed (Dziobek et al., 2006; Ha et al., 2013), and their use in future studies would represent an advance on the measures used in the studies included in this review. Future studies should also adopt more rigorous approaches to the measurement of eating pathology in children and adolescents, for instance by combining self-report instruments with interview measures or clinical diagnosis as given by specialist eating disorder clinicians. 
Furthermore, future research should investigate attachment and mentalization using clinical samples of children and adolescents, and should explore the impact of these variables on the process and outcome of treatment in child and adolescent eating disorders. Studies in the adult field have shown differential responsiveness to treatment approach based on attachment style (Illing et al., 2010) and mentalizing (Rothschild-Yakar et al., 2010a; Schulte-Rüther et al., 2012a). In this review, the study by Rothschild-Yakar et al. (2013) suggests there is variance in mentalization within clinical adolescent eating disorder samples. Future research should investigate whether variance in mentalization moderates treatment response in adolescent populations.

\subsection{Limitations}

A limitation of this review is that we could not rule out the possibility of publication bias, given that positive findings are more likely to be published than negative findings. The apparent relationship between attachment, mentalization and eating pathology may therefore be weaker than it appears.

\subsection{Conclusion}

The finding of an association between insecure attachment and eating pathology has been replicated several times in child and adolescent samples. The findings of the longitudinal studies in this review suggest that insecure attachment in early infancy is a distal risk factor with a relatively weak effect on adolescent eating pathology, whereas insecure attachment in pre-adolescence may predict increases in eating pathology more reliably. Currently, few studies have examined associations between 
mentalization and eating pathology in children and adolescents. The available evidence suggests that poor mentalization may be associated with eating pathology in both clinical and non-clinical samples. Future research in non-clinical samples should include prospective studies and experimental designs, to elucidate potential etiological pathways. Studies using clinical samples should investigate the roles played by attachment and mentalization in the process and outcome of treatment. Given the multi-factorial causation of eating disorders (Polivy \& Herman, 2002), any single variable is likely to have a complex relationship with eventual eating disorder pathology, in interaction with multiple other variables. The utility of the constructs of attachment and mentalization will therefore not be in providing a definitive account of etiology. By contrast, the value of these constructs to the eating disorder field will be in the extent to which they can inform innovations to treatment programs that lead to improved outcomes. On this basis, whilst the evidence base is currently very limited, attachment and mentalization represent potentially promising areas for further research. 


\subsection{Updated literature review}

\subsubsection{Studies of attachment and eating pathology}

There has been an increase in both the volume and quality of studies of attachment and eating pathology in childhood since the publication of the preceding systematic review, which are beginning to address some of the gaps identified in the evidence base.

\subsubsection{Reviews of attachment and eating pathology}

There have been two meta-analyses published on attachment and eating pathology, and a further meta-analysis supervised by the author of this thesis has been completed (Apostolidou, 2019) and is being prepared for publication (Apostolidou et al., in preparation).

A cross-sectional meta-analysis by (Faber et al., 2018) found small but significant correlations in the range of $r=0.2$ between attachment and eating pathology, using mostly non-clinical samples. In their moderation analyses, larger effect sizes for both attachment anxiety and avoidance were found for clinical samples as compared with non-clinical ones, although no such effect was found for an overall dimension of security. This cross-sectional approach was helpfully extended in the meta-analysis by (Cortés-García et al., 2019a), who examined mediators of the relationship between attachment and eating pathology across both clinical and non-clinical samples. Various mediators emerged as significant, with emotional regulation and depressive symptoms each explaining around $70 \%$ of the variance in the relationship between attachment and eating pathology. These findings suggest that the cross-sectional 
association between attachment and eating pathology takes place within a constellation of highly associated variables. Importantly for clinical practice, the evidence base does not yet provide a sufficient answer to the question of which of these variables - for instance attachment, emotion regulation difficulties or depressive symptoms - constitutes the most viable treatment target. One crucial gap in our understanding is the relationship between attachment and eating difficulties over time, although several new studies considered below speak to this issue.

Apostolidou (2019) completed a systematic review and meta-analysis comparing attachment in eating disorder samples compared to community controls, and investigated age, sampling method and quality of control group as moderators. The cognitive interpersonal maintenance model (Schmidt \& Treasure, 2006) suggests that insecure attachment, along with other interpersonal difficulties, could be maintaining factors for eating disorders, thereby hampering recovery. If true, a sampling bias might operate whereby persons with eating disorders high on insecure attachment may be more likely to remain ill. It was hypothesized that the impact of an eating disorder on social functioning could mean that insecure attachment is a consequence rather than cause of eating pathology, with older age and longer duration of illness leading to increasingly insecure attachment. Sampling method was also investigated as a moderator, for similar reasons. Studies that recruit chronically unwell patients, e.g. through convenience sampling, might show larger effect sizes than studies using consecutive sampling in community settings. Finally, the quality of the control group was investigated as a moderator, since most studies do not control for possible confounders such as psychopathology or emotional regulation. Moreover, poorly matched control groups could introduce bias, leading to inflated effect sizes. 
The study confirmed previous findings of large effect sizes for attachment insecurity between eating disorder and community control samples. There was no moderating effect for age, sampling method or quality of community controls. This suggests that the differences in attachment between eating disorder and community samples are not an artefact of methodological issues, nor do they appear to vary by age. Chronicity could not be investigated as a moderator due to a lack of reporting on this variable in included studies. Large effect sizes were found for both attachment avoidance and attachment anxiety, across both anorexia nervosa and bulimia nervosa samples. Apostolidou et al. (in preparation) recommend that future studies in this area need to control for psychopathology, report data on duration of illness, and employ longitudinal designs to understand the course of attachment security over time.

\subsubsection{Community studies of attachment and eating pathology}

Three recent studies have begun to shed light on the issue of change in attachment over time in adolescents. The most sophisticated of these are two studies by CortésGarcía et al. (2019b; 2020a) which used a cross-lagged panels design to investigate the relationship between eating pathology and attachment in a sample of 908 adolescent boys and girls across four time-points. At Time 1, the mean age of the sample was around age 10. The sample was then followed up every two years, meaning that at Time 4 the mean age was around 16. Eating pathology was measured with the ChEAT (Maloney et al., 1988), and attachment was measured with the Inventory of Parent and Peer Attachment (IPPA) (Armsden \& Greenberg, 1987a), which yields three scales (or inventories) for mother, father and peer attachment, each of which are comprised of three subscales (Communication, Trust and Alienation see Chapter 4 for more information). Analyses were conducted for all three attachment 
scales separately, and associations were also reported separately for girls and boys. The main finding was that attachment to mother was prospectively associated with disordered eating, such that more secure attachment predicted less disordered eating at the subsequent time point. By contrast, disordered eating did not predict insecure attachment to mother, with one exception, which was for girls only between Time 3 and Time 4 (ages 14 to 16 ).

Attachment to father predicted subsequent disordered eating only for girls between Time 1 and Time 2 (ages 10 to 12). The only significant prospective association between disorder eating and attachment to father was found for boys between Time 1 and Time 2, in the direction of more disordered eating predicting more secure attachment to father. For attachment to peers, the only significant prospective association was for boys between Time 1 and Time 2, with more secure attachment to peers predicting higher disordered eating. This is in keeping with the findings in the Le Grange et al. (2014) study reported earlier in this chapter. However, this first study did not examine the potential role of psychopathology in explaining the prospective association between insecure attachment and later eating pathology.

In a second study using this dataset, Cortés-García et al. (2020a) report a similar form of analysis that included measurement of self-reported depressive symptomatology in addition to attachment and eating pathology at each of the four time-points. Structural equation modelling was used to assess model fit for a cross-lagged panel model including data from the four time points, using a Dynamic Panel Model approach (Wichstrøm et al., 2017). Analyses using attachment to father resulted in poor model fit, whereas model fit indices for a Dynamic Panel Model using attachment to mother scores were good. Depressive symptoms, but not attachment to mother, were 
prospectively associated with eating pathology at each subsequent time point. In addition, insecure attachment to mother predicted increased depressive symptoms at each subsequent time point. The authors report several mediation paths between insecure attachment to mother at age 10 and disordered eating at age 16, using only the variables of attachment and depression. For instance, they report that $8.2 \%$ of the variance between insecure attachment at age 10 and disordered eating at 16 is explained by the following pathway: insecure attachment at age 10 predicts increased depressive symptoms at age 12, which predicts increased depressive symptoms at 14 , which predicts eating pathology at 16 .

Overall, the study suggests that depressive symptoms may represent a mediating variable between insecure attachment and eating pathology. However, the authors do not report whether the association between insecure attachment at age 10 and eating pathology at age 16 remained significant when controlling for depressive symptoms at age 10. Comparing the first and second studies (Cortés-García et al., 2019b; 2020a), it is striking that the prospective associations between attachment and eating pathology at the subsequent timepoint (e.g. Time 1 to Time 2) are only significant in the first study, which did not include depression. The authors suggest that promotion of secure attachment to mothers in the transition from middle childhood to adolescent could be a target for prevention, with attachment providing a potentially protective effect for both depression and in turn later eating pathology. Whilst interventions to promote secure attachment could quite plausibly lead to protective effects for child and adolescent mental health, it is unclear how these could be delivered at scale. Ultimately, experimental studies that seek to manipulate variables such as attachment and depression are needed both to establish that they represent modifiable targets for intervention, and to clarify which variables represent the most feasible targets. 
Van Durme et al. (2018) have also reported a longitudinal study of attachment and eating pathology in a sample of 397 community adolescents, with a mean age at Time 1 of 14 years of age. This study investigated associations between attachment anxiety and attachment avoidance at Time 1 with bulimic symptoms one year later (Time 2), whilst exploring the potential mediating effect of emotional control and rumination. The authors found both attachment avoidance and anxiety at Time 1 to be associated with bulimic symptoms at Time 2 . In their mediation analysis, which controlled for gender, adjusted body mass index and Time 1 bulimic symptoms, emotional control at Time 1 partially mediated the relationship between attachment avoidance and bulimic symptoms.

Another community study of note was conducted by Goossens et al. (2017) in a rare experimental design, which used the Trier Social Stress Test (Kirschbaum et al., 1993) to investigate the moderating role of affect on the association between disordered eating and insecure attachment. The authors hypothesised that increases in negative affect and/or decreases in positive affect after exposure to stress would result in decreases in body satisfaction in children with higher levels of attachment anxiety. The sample comprised a group of 81 children with a mean age of 12 . Body dissatisfaction was assessed before and after the laboratory-based induction of stress using the Trier Social Stress Test. The study found that children with high levels of attachment anxiety reported a decrease in body satisfaction, but only if the TSST-C led to a decrease in their positive affect. Finally, in a cross-sectional study of 167 adolescent girls with a mean age of 15, Goossens et al. (2019) found both attachment anxiety and attachment avoidance to play a moderating role in the relationship between thin ideal internalization and eating pathology. 


\subsubsection{Studies of attachment in adolescent eating disorder samples}

A lack of clinical studies was noted in our 2016 review (Jewell et al., 2016b). In recent years this gap in the literature has begun to be filled. In their systematic review and meta-analysis, Apostolidou et al. (in preparation) found several new studies of attachment in adolescent eating disorder samples compared with community controls. Using self-report measures of attachment, significant differences in attachment were found by Batista et al. (2018), who used the Experiences in Close RelationshipsRevised (Fraley et al., 2000) in a sample of 35 adolescents with AN and 35 Healthy Control (HC) adolescents. By contrast, Wallis et al. (2018), who used a brief version of the IPPA (Armsden and Greenberg, 1987), did not find any differences between their AN sample $(\mathrm{n}=54)$ and HC sample $(\mathrm{n}=49)$, either at baseline - when the AN group were starting FBT - or 14 months later, when the two samples again completed the IPPA. The 14-month follow-up represents 6 months after the end of treatment, which was 20 sessions of FBT.

Meanwhile, in studies employing interview measures of attachment, significant differences between adolescent eating disorder and control groups were found by Pace et al. (2016), who used the Adult Attachment Interview (George, Kaplan and Main, 1985), in a sample of 25 adolescents with AN, and $25 \mathrm{HC}$ individuals; and also by Attili et al. (2018) who used the Separation Anxiety Test (Wright et al., 1995) in a sample of 55 adolescents with eating disorders, and $80 \mathrm{HC}$ adolescents. Overall, these studies were rated as having a low risk of bias in most domains (e.g. sampling method, use of validated measures, blinding of assessors) (Apostolidou, 2019).

A cross-sectional study by Brochu et al. (2018) investigated the potential mediating roles of low self-esteem and depression in the relationship between attachment and 
eating pathology in a clinical sample of 186 adolescent females with anorexia nervosa. Using the IPPA (Armsden and Greenberg, 1987), the authors found all subscales (Alienation, Communication and Trust) of all three inventories (attachment to mother, father and peers) to correlate with the Eating Disorder Risk Composite score of the Eating Disorders Inventory (EDI-3; Garner, 2004). In the multiple regression analysis in which all subscales of the three IPPA inventories were entered, only the Alienation subscales of the mother and peer attachment scales remained as significantly associated with eating pathology. Mediation analyses were then conducted with these two scales only. For attachment to mother, low self-esteem and depression partially mediated the relationship between the alienation subscale and EDI-3 composite score; for attachment to peers, depression and self-esteem fully mediated the relationship between alienation and eating pathology.

Two published studies have also reported on change in attachment over time in clinical samples. Wallis et al. (2018), in the study just described using a sample receiving FBT, found that self-reported attachment to mothers and father reduced (with a small but significant effect size) between baseline and 6- month follow-up after session 20; this decline in attachment security took place in both the AN and $\mathrm{HC}$ samples, who as mentioned previously did not differ in terms of mean scores at either baseline or follow-up. Baudinet et al. (2020) have recently reported on change in attachment using the Attachment Style Questionnaire (Feeney et al., 1994) - the measure used in the main study of this thesis (Chapter 5) - in a sample of 131 adolescents with AN receiving intensive day-patient treatment. Measures were completed on entry to and discharge from the day-patient program, with a mean length of stay in the program of approximately three months. On discharge, there were statistically significant 
decreases of small effect size in two subscales that align with the concept of attachment avoidance: the Discomfort with Closeness (Cohen's $d=0.43$ ) and Relationships as Secondary (Cohen's d =0.32) scales. Interestingly, scores on the Preoccupation with Relationships scale, which aligns with the concept of attachment anxiety, increased (Cohen's $d=0.22$ ), representing greater attachment insecurity in this domain at discharge. Meanwhile, one other scale aligned with attachment anxiety, Need for Approval, did not change significantly. The authors did not report on the Confidence scale, which represents secure attachment, since the internal reliability of the scale was low in their sample.

\subsubsection{Studies of mentalization and eating pathology}

Since the publication of our systematic review (Jewell et al., 2016b), progress in the field of mentalization and eating pathology for adolescents has been somewhat slower than it has been for attachment. Two reviews have been published. Bora \& Köse (2016) undertook a systematic review and meta-analysis confined to studies comparing theory of mind in anorexia nervosa and bulimia nervosa with healthy controls. Effect sizes were larger in acute anorexia nervosa (Cohen's $d=0.67$ ) than in either recovered anorexia nervosa $(d=0.35)$ or bulimia nervosa $(d=0.34)$. Importantly in the context of this thesis, meta-regression analyses reported that longer duration of illness, but not age, were associated with more severe deficits in theory of mind.

Simonsen et al. (2020) also recently completed a systematic review and meta-analysis of mentalizing profile in individuals with eating disorders. They adopted a broad definition of mentalizing, including alexithymia, theory of mind, reflective function and mind-mindedness. The authors conclude that the mentalizing profile for individuals with an eating disorder is complex and varies across dimensions of the 
construct. The meta-analysis performed was restricted to studies of alexithymia only and found significantly higher levels of alexithymia in patients with anorexia nervosa compared to healthy controls (Standardized Mean Difference $=-1.57$ ). The authors characterise the overall literature as sparse and call for more research on mentalizing in different diagnostic groups with eating disorders. In their narrative synthesis they state that the evidence points to lower reflective function in individuals with eating disorders compared to HC samples. However, in the case of mentalizing about others, they state that the evidence suggests that the mentalizing ability of individuals with eating disorders appears to be comparable with those of community controls. Emerging evidence in adolescent eating disorder samples appears to bear this out.

In a recent study comparing adolescents with $\mathrm{AN}(\mathrm{n}=41)$ to a clinical sample with depression ( $\mathrm{n}=20)$ and an HC group $(\mathrm{n}=53)$, Rothschild-Yakar et al. (2019) found lower reflective function and higher alexithymia in the $\mathrm{AN}$ and depression groups compared with $\mathrm{HC}$, but no significant differences in theory of mind. Reflective function was assessed using the Reflective Function Scale (Fonagy et al., 1998) and theory of mind with the Reading the Mind in the Eyes task (RME) (Baron-Cohen et al., 2001b). Null findings in regard to theory of mind were also found by Bentz et al., (2017) and Leslie et al. (2020). Bentz et al. (2017) also used the RME task in their study of adolescents with first episode $(n=48)$ and recovered $(n=28)$ AN compared with HCs ( $n=41)$, whilst Leslie et al. (2020) investigated the neural correlates of theory of mind using the Frith-Happé animations (Abell et al., 2000). Leslie et al.'s study of young adults (mean age across groups of around 18 years of age) represents the largest sample to date for such studies, including an acute AN sample ( $n=67)$, a weight-restored AN sample $(n=49)$ and a HC sample $(n=70)$. 
A recent study by Cortés-García et al. (2020) includes data on both attachment and mentalizing in a sample of 568 adolescent psychiatric inpatients, divided into an eating disorder group $(n=128)$ and psychiatric control group $(n=440)$ compared to a HC group $(\mathrm{n}=184)$. Allocation to the eating disorder group was based on adolescents reporting two or more symptoms of anorexia nervosa or bulimia nervosa on a structured diagnostic interview schedule. The authors note the low prevalence of participants meeting full criteria for an eating disorder, although the precise number is not stated. As such, findings from the study cannot be taken to be representative of eating disorder samples. Nevertheless, the study found lower attachment security (lower scores on the coherence scale of the Child Attachment Interview (ShmueliGoetz et al., 2008b)) with greater hypermentalizing and lower hypomentalizing (assessed by the hypermentalizing and hypomentalizing scales of the Movie for Assessing Social Cognition (Dziobek et al., 2006a) in the eating disorder group relative to the $\mathrm{HC}$ group. The eating disorder and psychiatric control group did not differ from each other on measures of attachment or mentalizing.

Finally, a recent study by Schaumberg et al. (2020) investigated prospective associations between social communication difficulties in middle childhood and eating disorder symptoms/diagnoses in adolescence, utilising data from a large epidemiological, longitudinal study. At age 8, participants completed the faces subtest of the Diagnostic Analysis of Nonverbal Accuracy task (Nowicki \& Duke, 1994), which is a computerized task assessing the accuracy of facial emotion recognition using photographs. Scores on this task at age 8 were not associated with eating disorder pathology or diagnoses during adolescence, but there were significant associations for two misattributions on the task. Greater misattributions of sad faces and angry faces 
were associated with a greater likelihood of purging and AN diagnosis, respectively, for girls at age 14 .

\subsubsection{Summary of the updated literature}

Recent studies provide support to the conclusions drawn in our systematic review (Jewell et al., 2016b) that attachment represents a risk factor for eating pathology. Studies with clinical populations have now confirmed that attachment security is lower in adolescent eating disorder samples, in keeping with findings from the adult literature. The association between attachment and eating pathology is robust and of a small effect size when effect sizes are pooled via meta-analysis. However, there is mounting evidence that this association may be mediated by depressive symptoms. Evidence of mentalizing deficits in adolescent eating disorder samples is not straightforward: reflective function appears lower in clinical samples than HC groups, but theory of mind appears not to be impaired. Studies of attachment and mentalizing in the process of treatment for adolescent eating disorders are still needed to investigate the value of these concepts in the clinical context. 
Chapter 4 - Attachment measures in middle childhood and adolescence: A systematic review of measurement properties

This chapter is presented as a published journal article and is an exact copy of the following publication:

Jewell, T., Gardner, T., Susi, K., Watchorn, K., Coopey, E., Simic, M., Fonagy, P. and Eisler, I. (2019). Attachment measures in middle childhood and adolescence: a systematic review of measurement properties. Clinical Psychology Review, 68, 7182. 


\subsection{Abstract}

Background: Attachment theory proposes that humans develop representations of self and other in early childhood which are relatively stable across the life-course, and play a key role in psychological adaptation. However, to date, the psychometric properties of attachment measures in middle childhood and adolescence have not been evaluated in a systematic review.

Method: A systematic review (PROSPERO ID: CRD42017057772) was conducted using COSMIN criteria. Two researchers independently searched MEDLINE, PsychINFO and Embase databases for relevant articles.

Results: Fifty-three studies were included in the review. The methodological quality of studies was typically fair or poor, with only a smaller number of studies being rated as of good or excellent quality. The measurement properties of attachment measures in this age group were frequently rated as inadequate according to COSMIN criteria. The Child Attachment Interview (CAI) has the best psychometric properties of the interview and projective measures, and the Inventory of Parent and Peer Attachment (IPPA) the best evidence of the self-report measures. Overall, the evidence for the CAI and IPPA included both positive and negative findings relating to adequacy of measurement properties.

Conclusions: Attachment measures in middle childhood and adolescence currently have limited evidence for the adequacy of their psychometric properties. 


\section{$\underline{\text { 4.1.1 Abbreviations }}$}

Self-report measures: $\mathrm{AAQ}=$ Adolescent Attachment Questionnaire, AFAS = Adolescent Friendship Attachment Scale, AFAS-SF = Adolescent Friendship Attachment Scale - Short Form, AUAQ $=$ Adolescent Unresolved Attachment Questionnaire, ASQ = Attachment Style Questionnaire, ECR-RC = Experiences in Close Relationships - Revised for Children, ECR-RS = Experiences in Close Relationships - Relationship Structures, Experiences in Close Relationships - Revised

- General Short Form (ECR-R- GSF), IPPA = Inventory of Parent and Peer Attachment, IPPA-B = Inventory of Parent and Peer Attachment- Brief version, IPPA45 = Inventory of Parent and Peer Attachment - 45, IPPA-R = Inventory of Parent and Peer Attachment - Revised, IPPA-S = Inventory of Parent and Peer Attachment Short Form, PIML = People in my Life

Observer-rated measures: $\mathrm{AAI}=$ Adult Attachment Interview, AAP $=$ Adult Attachment Projective, AAPQ = Adolescent Attachment Prototype Questionnaire, $\mathrm{AQ}-\mathrm{A}=$ Attachment Questionnaire - Adolescent Version, ASCT = Attachment Story Completion Task, ASA $=$ Attachment Script Assessment, BND $=$ Bird's Nest Drawing, CYTM = Can You Tell Me?, CAPA = Child Attachment and Play Assessment, $\mathrm{CAI}=$ Child Attachment Interview, $\mathrm{CMSSB}=$ Computerized MacArthur Story Stem Battery, CMCAST = Computerized Manchester Child Attachment Story Task, FAI $=$ Family Attachment Interview, FFI $=$ Friends and Family Interview, MCAST $=$ Manchester Child Attachment Story Task, SAA = School-age Assessment of Attachment, SBST = Secure Base Script Test, SAT = Separation Anxiety Test. 


\subsection{Introduction}

Attachment theory constitutes an evolving body of work developed by dozens of researchers and theoreticians over approximately sixty years. Two propositions are central to the theory: (1) patterns of attachment developed in infancy are relatively stable across the lifespan; (2) attachment patterns can help to explain the development of psychopathology (Bowlby, 1973, 1980). Both propositions can be tested empirically, but this requires the presence of valid and reliable attachment measures. Evidence points to attachment as a risk factor for the development of a range of psychopathology, including aggression and externalising behaviour (Fearon et al., 2010), internalizing disorders (Groh et al., 2012), eating pathology (Jewell et al., 2016b; Caglar-Nazali et al., 2014) and suicidality (Fergusson et al., 2000). However, the power of attachment to predict later psychopathology is generally weak, raising doubts about its centrality as a causal factor (Fonagy, Luyten \& Allison, 2015). Moreover, there have been concerns that measures of attachment in childhood may have high levels of error (Fearon and Roisman, 2017), thus hampering efforts to understand the role of attachment in psychopathology.

Middle childhood and adolescence constitute developmental phases for which no 'gold standard' measures exist (Bosmans and Kerns, 2015). The aim of this study is to provide the first ever systematic review of the psychometric properties of all measures of attachment in middle childhood and adolescence. To understand the theoretical underpinnings of such measures, it is necessary to provide an account of the development of measurement approaches to infant and adult attachment. We note that the psychometric properties of these instruments have yet to be subjected to a systematic review, but recent narrative reviews of infant and adult attachment 
measures have been provided by Solomon and George (2016) and Crowell, Fraley and Roisman (2016) respectively.

\subsubsection{Measuring attachment in infancy and adulthood}

The empirical assessment of human attachment began with Ainsworth, Blehar, Waters, \& Wall's (1978) 'strange situation procedure' (SSP), in which trained observers rated infant behaviour in response to separation from and reunion with their primary caregiver. Although infants were rated across various scales, a discriminant function analysis suggested two underlying attachment dimensions, named avoidance and anxiety. Noting the clustering of the data in three groups resulted in the naming of three attachment categories: A, B and C, which came to be known as insecureavoidant, secure and insecure-ambivalent. Later, Main and Solomon (1986) identified a fourth category in the SSP, disorganised attachment (D). The resulting four-category 'ABCD' paradigm directly informed the development of the Adult Attachment Interview (AAI) (George, Kaplan \& Main, 1985). This was originally developed in an attempt to explain the SSP classifications of infants by identifying differences in mother's representations of their own childhood experiences of being cared for. The four AAI categories - secure, dismissing, preoccupied and disorganised - were conceived of as explaining secure, avoidant, resistant and disorganised attachment patterns (respectively) in infants.

In a separate development, researchers such as Hazan and Shaver (1987) developed self-report measures of romantic attachment style. These measures were based on putative parallels between adults' ways of relating in romantic relationships and the 
concept of infant attachment categories. Brennan, Clarke and Shaver (1998) undertook a factor analysis of a large pool of items taken from self-report attachment style measures, and identified two underlying factors, avoidance and anxiety, echoing the dimensions identified by Ainsworth et al. (1978). In recent years, taxometric analyses of both the SSP (Fraley and Spieker, 2003) and the AAI (Fraley and Roisman, 2014) have also suggested that attachment is distributed across these two dimensions, rather than falling within categories. Nevertheless, concordance between AAI categories and self-reported attachment is trivial to low (Roisman, 1997).

Thus, the two main approaches to measuring adult attachment may be tapping into related but distinct constructs, although the precise boundaries of the adult attachment construct are hard to operationalize (Allen et al., 2005). The concept of adult attachment style as tapped by self-report-measures has been defined as a constellation of knowledge, expectations and insecurities that people hold about themselves and their close relationships (Fraley and Roisman, 2018). By contrast, the AAI seems to access internal working models of childhood caregiving experiences (Stein et al., 1998), although Allen and Miga (2010) have suggested that the AAI may be best conceived as a measure of emotion regulation in the context of discussions about caregiving experiences.

\subsubsection{Measuring attachment in middle childhood and adolescence}

As can be gleaned from this overview, whilst measures of attachment exist for both infancy and adulthood, there are enormous differences not only in measurement approach, but also in the latent construct that is being assessed. In infancy, an 
observational measure is used to assess behaviour in young children who are developmentally reliant on their caregivers for survival, and are at a very early stage in their emotional, cognitive and social development. By contrast, attachment measures in adulthood assess mental representations, expressed through language, in adults who have acquired formal operational thinking (Inhelder and Piaget, 1958), and whose close relationships serve quite different functions, potentially including reproduction, child-rearing and emotional support. Approaches to assessing attachment in middle childhood and adolescence therefore must take into account the 'moving target' of child development, both in terms of the evolving function of the attachment system, and the changing abilities of the child.

Researchers have tried to access children's attachment representations using three main methods. Firstly, various self-report measures have been developed, including specific measures developed for middle childhood (Kerns, 1996), measures developed with both adults and adolescents in mind (Feeney et al., 1994), and downward extensions of adult romantic attachment measures (e.g. Brenning et al., 2011). Secondly, interview approaches informed by the AAI have been developed (ShmueliGoetz et al., 2008). Finally, some researchers have developed projective measures, in which children's stories and play in response to attachment-related prompts (e.g. Green et al., 2000; Cassidy, 1990) are rated by trained coders. Thus far, attempts to critically appraise the measurement properties of these various measures have been limited. The National Institute for Health and Care Excellence (NICE) (2016) conducted the most thorough review so far but excluded self-report measures. Kerns et al. (2005) and Wilson and Wilkinson (2012) have also conducted reviews but these did not include ratings of the methodological quality of studies. 


\subsubsection{Rationale for this review}

Attachment theory constitutes a highly important paradigm within the field of child development and psychopathology, resulting in thousands of empirical papers. However, the reliability and validity of measures is fundamental to the conduct and interpretation of this body of research.

\section{$\underline{4.2 .4 \text { Objective }}$}

Our primary aim in undertaking this review is to make recommendations about which attachment measures have the best psychometric properties, thereby providing a guide to researchers and clinicians in the field. We will also identify gaps in the evidence and make recommendations about promising avenues for future research.

\subsection{Method}

\subsubsection{Literature search}

This systematic review was registered with PROSPERO (CRD 42017057772) and completed in accordance with PRISMA guidelines (Moher et al., 2009). Two independent researchers searched MEDLINE, PsychINFO and Embase databases for relevant articles up to the end of June 2017. Eligibility criteria were: (1) English language; (2) published in a peer-reviewed journal; (3) aim of study is to develop a measure, or evaluate the properties of a measure, that assesses attachment in children or adolescents using either interview, task, projective method or self-report; (4) participants in the study are aged between 6 and 18 years inclusive; (5) measure is theoretically derived from attachment theory. The search strategy was constructed by TG, and refined by TJ, over three waves of literature searching in February 2016, 
November 2016 and June 2017. Our search strategy is publicly available at the PROSPERO protocol registration for this review (CRD42017057772).

\subsubsection{Data extraction}

This study used the COSMIN checklist (De Vet et al, 2011), a tool for systematic reviews of measurement properties. Data were extracted using templates from the COSMIN tool. Characteristics of studies (e.g. sample size, age of participants) can be found in Appendices A and B for observer-rated measures and self-report measures respectively. Characteristics of the measures themselves (e.g. number of items and scales) can be found in Tables 4.1 and 4.2.

\section{$\underline{4.3 .3 \text { Assessment of measurement properties }}$}

Pairs of reviewers independently assessed each study using the COSMIN checklist for the following criteria: internal consistency, test-retest and inter-rater reliability, content validity, hypotheses testing (i.e. construct validity), cross cultural validity and criterion validity. Measurement error and responsiveness to change are part of the COSMIN checklist but were not included in this review since no studies were found addressing these measurement properties.

Each study was rated by a pair of reviewers. TJ rated all papers, and TG, KW, KS and EC rated approximately a quarter of papers each. Disagreements were resolved by PF. Each measurement property was rated on a four-point rating scale (poor, fair, good, excellent) for methodological quality of the study, thereby assessing risk of bias within studies. Each measurement property also received a rating for the adequacy of the 
measurement property (assigned as ‘+', '-' or '?'). COSMIN criteria for the adequacy of measurement properties can be found in Table 4.3. Ratings of the methodological quality and adequacy of measurement properties within individual studies are in Appendices C and D (observer-rated and self-report measures respectively).

\section{$\underline{\text { 4.3.4 Data synthesis }}$}

A synthesis of the strength of evidence for each measurement property was conducted for all measures other than those which only achieved ratings of poor methodological quality (Tables 4.4 and 4.5: observer-rated and self-report measures respectively). Criteria used to define the strength of evidence can be found in Appendix E. Finally, we incorporated a brief narrative summary of strengths and weaknesses of each measure into Tables 4.1 and 4.2, so that researchers and clinicians can rapidly appraise the characteristics of different measures 'at a glance'. 
Table 4.1 Characteristics, strengths and weaknesses of observer-rated measures

\begin{tabular}{|c|c|c|c|c|c|c|c|}
\hline $\begin{array}{l}\text { Measure } \\
\text { (Abbreviation) }\end{array}$ & \# Questions & $\begin{array}{l}\text { \# Scales (range of } \\
\text { scores) }\end{array}$ & $\begin{array}{l}\text { Admin. } \\
\text { time }\end{array}$ & $\begin{array}{l}\text { Final categories or } \\
\text { dimensions yielded }\end{array}$ & $\begin{array}{l}\text { Focus of } \\
\text { measure }\end{array}$ & Strengths & Weaknesses \\
\hline $\begin{array}{l}\text { Adult Attachment } \\
\text { Interview } \\
\text { (AAI) }\end{array}$ & 20 questions & $\begin{array}{l}17 \text { subscales: } 5 \\
\text { experience scales (e.g. } \\
\text { loving/unloving); } 3 \text { states } \\
\text { of mind regarding } \\
\text { parents (e.g. idealising); } \\
9 \text { for state of mind with } \\
\text { respect to attachment } \\
\text { (e.g. coherence) (1-9) }\end{array}$ & 1 hour & $\begin{array}{l}\text { Categories: Secure, } \\
\text { Dismissing, Preoccupied, } \\
\text { Unresolved/Disorganized } \\
\text {, Cannot Classify }\end{array}$ & $\begin{array}{l}\text { Adult } \\
\text { attachment } \\
\text { status }\end{array}$ & $\begin{array}{l}\text { Well-validated in } \\
\text { adults thus good } \\
\text { choice for older } \\
\text { adolescents. }\end{array}$ & $\begin{array}{l}\text { Lengthy administration and } \\
\text { coding time; known to be hard } \\
\text { to achieve reliability for } \\
\text { coding. }\end{array}$ \\
\hline $\begin{array}{l}\text { Adult Attachment } \\
\text { Projective } \\
\text { (AAP) }\end{array}$ & $\begin{array}{l}8 \text { line } \\
\text { drawings; } 1 \\
\text { warm-up } \\
\text { and } 7 \\
\text { attachment } \\
\text { scenes }\end{array}$ & $\begin{array}{l}\text { Agency of self, } \\
\text { connectedness, } \\
\text { synchrony, deactivation, } \\
\text { cognitive disconnection, } \\
\text { segregated systems (?) }\end{array}$ & $?$ & $\begin{array}{l}\text { Categories: Secure, } \\
\text { Dismissing, Preoccupied, } \\
\text { Unresolved }\end{array}$ & $\begin{array}{l}\text { Adult } \\
\text { attachment } \\
\text { status }\end{array}$ & $\begin{array}{l}\text { Good inter-rater } \\
\text { reliability. }\end{array}$ & $\begin{array}{l}\text { Training for reliability is } \\
\text { lengthy ( } 9 \text { days) thereby } \\
\text { limiting its use in clinical } \\
\text { settings. }\end{array}$ \\
\hline $\begin{array}{l}\text { Adolescent } \\
\text { Attachment } \\
\text { Prototype } \\
\text { Questionnaire } \\
\text { (AAPQ) }\end{array}$ & $\begin{array}{l}4 \text { attachment } \\
\text { prototype } \\
\text { descriptions } \\
\text { rated by } \\
\text { clinician }\end{array}$ & $\begin{array}{l}\text { Secure, Dismissing, } \\
\text { Preoccupied, Incoherent/ } \\
\text { Disorganized (1-5 Likert } \\
\text { scale) }\end{array}$ & $?$ & $\begin{array}{l}\text { Dimensional and } \\
\text { categorical ratings: } \\
\text { Secure, Dismissing, } \\
\text { Preoccupied, } \\
\text { Incoherent/Disorganized }\end{array}$ & $\begin{array}{l}\text { Generalised } \\
\text { attachment } \\
\text { status }\end{array}$ & $\begin{array}{l}\text { Straightforward } \\
\text { clinician-rated } \\
\text { measure; no need to } \\
\text { transcribe. }\end{array}$ & $\begin{array}{l}\text { Inadequate construct validity } \\
\text { (hypothesis testing). }\end{array}$ \\
\hline $\begin{array}{l}\text { Attachment } \\
\text { Questionnaire - } \\
\text { Adolescent Version } \\
\text { (AQ-A) }\end{array}$ & $\begin{array}{l}37 \text { clinician- } \\
\text { rated items }\end{array}$ & $\begin{array}{l}\text { Secure, Dismissing, } \\
\text { Preoccupied, Incoherent/ } \\
\text { Disorganized } \\
\text { (1-7 Likert scale) }\end{array}$ & $?$ & $\begin{array}{l}\text { Dimensional and } \\
\text { categorical ratings: } \\
\text { Secure, Dismissing, } \\
\text { Preoccupied, } \\
\text { Incoherent/Disorganized }\end{array}$ & $\begin{array}{l}\text { Generalised } \\
\text { attachment } \\
\text { status }\end{array}$ & $\begin{array}{l}\text { Straightforward } \\
\text { clinician-rated } \\
\text { measure; no need to } \\
\text { transcribe. }\end{array}$ & $\begin{array}{l}\text { No data on construct validity } \\
\text { (hypothesis testing). }\end{array}$ \\
\hline $\begin{array}{l}\text { Attachment Story } \\
\text { Completion Task } \\
\text { (ASCT) }\end{array}$ & $\begin{array}{l}\text { Warm-up, } \\
\text { then } 5 \text { story } \\
\text { stems }\end{array}$ & $\begin{array}{l}\text { Secure, Avoidant, } \\
\text { Ambivalent, } \\
\text { Disorganized (1-5) }\end{array}$ & $?$ & $\begin{array}{l}\text { Categories: Secure, } \\
\text { Avoidant, Ambivalent, } \\
\text { Disorganized }\end{array}$ & $\begin{array}{l}\text { Parent } \\
\text { attachment }\end{array}$ & $\begin{array}{l}\text { Suitable for middle } \\
\text { childhood. }\end{array}$ & $\begin{array}{l}\text { Inadequate inter-rater } \\
\text { reliability and construct } \\
\text { validity (hypothesis testing); } \\
\text { content and scoring methods } \\
\text { have differed between studies. }\end{array}$ \\
\hline
\end{tabular}


Table 4.1 Characteristics, strengths and weaknesses of observer-rated measures (continued)

\begin{tabular}{|c|c|c|c|c|c|c|c|}
\hline $\begin{array}{l}\text { Measure } \\
\text { (Abbreviation) }\end{array}$ & \# Questions & $\begin{array}{l}\text { \# Scales (range of } \\
\text { scores) }\end{array}$ & $\begin{array}{l}\text { Admin. } \\
\text { time }\end{array}$ & $\begin{array}{l}\text { Final categories or } \\
\text { dimensions yielded }\end{array}$ & $\begin{array}{l}\text { Focus of } \\
\text { measure }\end{array}$ & Strengths & Weaknesses \\
\hline $\begin{array}{l}\text { Attachment Script } \\
\text { Assessment (ASA) }\end{array}$ & $\begin{array}{l}4 \text { stories } \\
\text { ( } 2 \text { mother, } 2 \\
\text { father) }\end{array}$ & $\begin{array}{l}\text { Secure base script } \\
\text { knowledge scale }(1-7)\end{array}$ & $?$ & $\begin{array}{l}\text { Continuous secure base } \\
\text { script knowledge score. }\end{array}$ & $\begin{array}{l}\text { Parent } \\
\text { attachment }\end{array}$ & $\begin{array}{l}\text { Good inter-rater } \\
\text { reliability; simpler } \\
\text { scoring method } \\
\text { compared with other } \\
\text { observer-rated } \\
\text { measures. }\end{array}$ & $\begin{array}{l}\text { Requires further study to } \\
\text { explore convergent validity } \\
\text { with other established } \\
\text { measures; test retest reliability } \\
\text { is unknown. }\end{array}$ \\
\hline $\begin{array}{l}\text { Bird's Nest } \\
\text { Drawing (BND) }\end{array}$ & $\begin{array}{l}\text { Task: } \\
\text { Drawing of a } \\
\text { bird's nest }\end{array}$ & $\begin{array}{l}\text { Vitality, General } \\
\text { pathology, Bizareness, } \\
\text { Optimism }\end{array}$ & $?$ & $\begin{array}{l}\text { Categories: Secure, } \\
\text { Insecure }\end{array}$ & $\begin{array}{l}\text { Parent } \\
\text { attachment }\end{array}$ & $\begin{array}{l}\text { Good inter-rater } \\
\text { reliability; non-verbal } \\
\text { means of assessment. }\end{array}$ & $\begin{array}{l}\text { Inadequate construct validity; } \\
\text { no evidence available for most } \\
\text { psychometric properties. }\end{array}$ \\
\hline $\begin{array}{l}\text { Can You Tell Me? } \\
\text { (CYTM) }\end{array}$ & 22 questions & N/A & 40 mins & $\begin{array}{l}10 \text { categories based on } \\
\text { DMM attachment } \\
\text { strategies }\end{array}$ & $\begin{array}{l}\text { Parent } \\
\text { attachment }\end{array}$ & $\begin{array}{l}\text { Semi-structured } \\
\text { interview format can } \\
\text { elicit rich clinical } \\
\text { data }\end{array}$ & $\begin{array}{l}\text { Inadequate psychometric } \\
\text { properties; lengthy } \\
\text { administration and coding }\end{array}$ \\
\hline $\begin{array}{l}\text { Child Attachment } \\
\text { and Play } \\
\text { Assessment } \\
\text { (CAPA) }\end{array}$ & $\begin{array}{l}10 \text { questions, } \\
8 \text { stems }\end{array}$ & N/A & $?$ & $\begin{array}{l}6 \text { categories based on } \\
\text { DMM attachment } \\
\text { strategies }\end{array}$ & $\begin{array}{l}\text { Parent } \\
\text { attachment }\end{array}$ & $\begin{array}{l}\text { Semi-structured } \\
\text { interview format can } \\
\text { elicit rich clinical } \\
\text { data. }\end{array}$ & $\begin{array}{l}\text { Inadequate psychometric } \\
\text { properties; lengthy } \\
\text { administration and coding. }\end{array}$ \\
\hline $\begin{array}{l}\text { Child Attachment } \\
\text { Interview } \\
\text { (CAI) }\end{array}$ & 15 questions & $\begin{array}{l}\text { Involving anger, } \\
\text { Idealization, Dismissal, } \\
\text { Resolution of conflict, } \\
\text { Balance of positive and } \\
\text { negative references to } \\
\text { attachment figures, } \\
\text { Overall coherence, } \\
\text { Emotional openness, Use } \\
\text { of examples (1-9) }\end{array}$ & $\begin{array}{l}20-80 \\
\operatorname{mins}\end{array}$ & $\begin{array}{l}\text { Categories: Secure, } \\
\text { Dismissing, Preoccupied, } \\
\text { Disorganized }\end{array}$ & $\begin{array}{l}\text { Mother and } \\
\text { father } \\
\text { attachment }\end{array}$ & $\begin{array}{l}\text { Overall the best- } \\
\text { validated observer- } \\
\text { rated measure of } \\
\text { attachment in middle } \\
\text { childhood and } \\
\text { adolescence; semi- } \\
\text { structured interview } \\
\text { format can elicit rich } \\
\text { clinical data. }\end{array}$ & $\begin{array}{l}\text { Lengthy administration and } \\
\text { coding time; known to be hard } \\
\text { to achieve reliability for } \\
\text { coding; inter-rater reliability is } \\
\text { inadequate in most studies. }\end{array}$ \\
\hline
\end{tabular}


Table 4.1 Characteristics, strengths and weaknesses of observer-rated measures (continued)

\begin{tabular}{|c|c|c|c|c|c|c|c|}
\hline $\begin{array}{l}\text { Measure } \\
\text { (Abbreviation) }\end{array}$ & \# Questions & $\begin{array}{l}\text { \# Scales (range of } \\
\text { scores) }\end{array}$ & $\begin{array}{l}\text { Admin. } \\
\text { time }\end{array}$ & $\begin{array}{l}\text { Final categories or } \\
\text { dimensions yielded }\end{array}$ & $\begin{array}{l}\text { Focus of } \\
\text { measure }\end{array}$ & Strengths & Weaknesses \\
\hline $\begin{array}{l}\text { Computerized } \\
\text { MacArthur Story } \\
\text { Stem Battery } \\
\text { (CMSSB) }\end{array}$ & 6 story stems & $\begin{array}{l}\text { Avoidance, Coherence, } \\
\text { intentionality }(1-12)\end{array}$ & $?$ & $\begin{array}{l}3 \text { dimensions: } \\
\text { Avoidance, Coherence, } \\
\text { intentionality }\end{array}$ & $\begin{array}{l}\text { Parent } \\
\text { attachment }\end{array}$ & $\begin{array}{l}\text { Computerised format } \\
\text { could allow for easier } \\
\text { administration to } \\
\text { larger research } \\
\text { samples. }\end{array}$ & $\begin{array}{l}\text { Limited evidence of adequate } \\
\text { psychometric properties. }\end{array}$ \\
\hline $\begin{array}{l}\text { Computerized } \\
\text { Manchester Child } \\
\text { Attachment Story } \\
\text { Task (CMCAST) }\end{array}$ & 4 story stems & N/A & $?$ & $\begin{array}{l}\text { Categories: Secure, } \\
\text { Dismissing, Preoccupied, } \\
\text { Disorganized }\end{array}$ & $\begin{array}{l}\text { Parent } \\
\text { attachment }\end{array}$ & $\begin{array}{l}\text { Computerised format } \\
\text { could allow for easier } \\
\text { administration to } \\
\text { larger research } \\
\text { samples. }\end{array}$ & $\begin{array}{l}\text { Inadequate inter-rater } \\
\text { reliability. }\end{array}$ \\
\hline $\begin{array}{l}\text { Family Attachment } \\
\text { Interview } \\
\text { (FAI) }\end{array}$ & $?$ & $\begin{array}{l}\text { Secure, Fearful, } \\
\text { Preoccupied, Dismissing } \\
(1-9)\end{array}$ & 1-2 hours & $\begin{array}{l}\text { Categories: Secure, } \\
\text { Fearful, Preoccupied, } \\
\text { Dismissing }\end{array}$ & $\begin{array}{l}\text { Generalized } \\
\text { attachment } \\
\text { style }\end{array}$ & $\begin{array}{l}\text { Semi-structured } \\
\text { interview format can } \\
\text { elicit rich clinical } \\
\text { data. }\end{array}$ & $\begin{array}{l}\text { Inadequate psychometric } \\
\text { properties; lengthy } \\
\text { administration and coding. }\end{array}$ \\
\hline $\begin{array}{l}\text { Friends and Family } \\
\text { Interview (FFI) }\end{array}$ & 27 questions & $\begin{array}{l}\text { Coherence, } \\
\text { Mentalization, Secure } \\
\text { base availability, Social } \\
\text { competence, School } \\
\text { competence, Self-esteem, } \\
\text { Peer relations, Sibling } \\
\text { relations, Anxieties and } \\
\text { defence, Differentiations } \\
\text { of parental } \\
\text { representations, } \\
\text { Attachment } \\
\text { classifications, Non- } \\
\text { verbal codes (1-4) }\end{array}$ & $?$ & $\begin{array}{l}\text { Categories: Secure- } \\
\text { autonomous, Insecure- } \\
\text { dismissing, Insecure- } \\
\text { preoccupied, } \\
\text { Disoriented-disorganized }\end{array}$ & $\begin{array}{l}\text { Parents, } \\
\text { siblings and } \\
\text { peers }\end{array}$ & $\begin{array}{l}\text { Semi-structured } \\
\text { interview format can } \\
\text { elicit rich clinical } \\
\text { data. }\end{array}$ & $\begin{array}{l}\text { Inadequate psychometric } \\
\text { properties; lengthy } \\
\text { administration and coding. }\end{array}$ \\
\hline
\end{tabular}


Table 4.1 Characteristics, strengths and weaknesses of observer-rated measures (continued)

\begin{tabular}{|c|c|c|c|c|c|c|c|}
\hline $\begin{array}{l}\text { Measure } \\
\text { (Abbreviation) }\end{array}$ & \# Questions & $\begin{array}{l}\text { \# Scales (range of } \\
\text { scores) }\end{array}$ & $\begin{array}{l}\text { Admin. } \\
\text { time }\end{array}$ & $\begin{array}{l}\text { Final categories or } \\
\text { dimensions yielded }\end{array}$ & $\begin{array}{l}\text { Focus of } \\
\text { measure }\end{array}$ & Strengths & Weaknesses \\
\hline $\begin{array}{l}\text { Goal-Corrected } \\
\text { Partnership } \\
\text { Adolescent Coding } \\
\text { System (GPACS) }\end{array}$ & $\begin{array}{l}\text { Task: two 5- } \\
\text { min conflict } \\
\text { discussions } \\
\text { (1 topic } \\
\text { chosen by } \\
\text { adolescent, } 1 \\
\text { topic chosen } \\
\text { by } \\
\text { caregiver) }\end{array}$ & $\begin{array}{l}\text { Collaborative } \\
\text { communication, } \\
\text { Warmth/valuing, } \\
\text { Caregiver validation of } \\
\text { adolescent's voice, } \\
\text { Caregiver's } \\
\text { hostile/punitive/devaluin } \\
\text { g behaviour, Caregiver's } \\
\text { role confused behaviour, } \\
\text { Caregivers' odd or out- } \\
\text { of-context/ contradictory } \\
\text { behaviour, Caregiver's } \\
\text { distracted, disoriented, or } \\
\text { inwardly absorbed } \\
\text { behaviour, Adolescent } \\
\text { advances own view, } \\
\text { Adolescent's hostile, } \\
\text { punitive, or devaluing } \\
\text { behaviour, Adolescent's } \\
\text { caregiving, organizing, } \\
\text { or entertaining } \\
\text { behaviour, Adolescent's } \\
\text { odd, out-of-context, or } \\
\text { contradictory behaviour, } \\
\text { Adolescent's distracted, } \\
\text { disoriented, or inwardly } \\
\text { absorbed behavior }\end{array}$ & 10 mins & $\begin{array}{l}\text { Dimensions: Security, } \\
\text { Role confused, Hostile, } \\
\text { Disorganized }\end{array}$ & $\begin{array}{l}\text { Observed } \\
\text { parent- } \\
\text { adolescent } \\
\text { interaction }\end{array}$ & $\begin{array}{l}\text { Observational } \\
\text { measure with dyadic } \\
\text { focus; brief } \\
\text { administration time; } \\
\text { adequate inter-rater } \\
\text { reliability. }\end{array}$ & Inadequate construct validity. \\
\hline
\end{tabular}


Table 4.1 Characteristics, strengths and weaknesses of observer-rated measures (continued)

\begin{tabular}{|c|c|c|c|c|c|c|c|}
\hline $\begin{array}{l}\text { Measure } \\
\text { (Abbreviation) }\end{array}$ & \# Questions & $\begin{array}{l}\text { \# Scales (range of } \\
\text { scores) }\end{array}$ & $\begin{array}{l}\text { Admin. } \\
\text { time }\end{array}$ & $\begin{array}{l}\text { Final categories or } \\
\text { dimensions yielded }\end{array}$ & $\begin{array}{l}\text { Focus of } \\
\text { measure }\end{array}$ & Strengths & Weaknesses \\
\hline $\begin{array}{l}\text { Manchester Child } \\
\text { Attachment Story } \\
\text { Task (MCAST) }\end{array}$ & $\begin{array}{l}6 \text { vignettes } \\
\text { (1 warm-up, } \\
5 \text { attachment } \\
\text { vignettes })\end{array}$ & $\begin{array}{l}33 \text { scales (most on 9- } \\
\text { point scales) }\end{array}$ & $\begin{array}{l}20-30 \\
\text { mins }\end{array}$ & $\begin{array}{l}\text { Categories: Secure, } \\
\text { Dismissing, Preoccupied, } \\
\text { Disorganised, Cannot } \\
\text { Classify }\end{array}$ & $\begin{array}{l}\text { Parent } \\
\text { attachment }\end{array}$ & $\begin{array}{l}\text { Suitable for younger } \\
\text { end of middle } \\
\text { childhood. }\end{array}$ & $\begin{array}{l}\text { Psychometric properties } \\
\text { generally inadequate. }\end{array}$ \\
\hline $\begin{array}{l}\text { School-age } \\
\text { Assessment of } \\
\text { Attachment (SAA) }\end{array}$ & $\begin{array}{l}7 \text { picture } \\
\text { cards }\end{array}$ & N/A & $?$ & $\begin{array}{l}13 \text { categories based on } \\
\text { DMM attachment } \\
\text { strategies }\end{array}$ & $\begin{array}{l}\text { Parent } \\
\text { attachment }\end{array}$ & $\begin{array}{l}\text { Semi-structured } \\
\text { interview format can } \\
\text { elicit rich clinical } \\
\text { data. }\end{array}$ & $\begin{array}{l}\text { Inadequate psychometric } \\
\text { properties; lengthy } \\
\text { administration and coding. }\end{array}$ \\
\hline $\begin{array}{l}\text { Secure Base Script } \\
\text { Test (SBST) }\end{array}$ & $\begin{array}{l}5 \text { sets of } \\
\text { word } \\
\text { prompts (1 } \\
\text { warm-up, } 2 \\
\text { parents, } 2 \\
\text { best friends) }\end{array}$ & Scriptedness (1-7) & $\begin{array}{l}8-20 \\
\operatorname{mins}\end{array}$ & $\begin{array}{l}1 \text { dimension: } 7 \text {-point } \\
\text { Scriptedness score }\end{array}$ & $\begin{array}{l}\text { Generalized } \\
\text { attachment } \\
\text { script }\end{array}$ & $\begin{array}{l}\text { Relatively brief } \\
\text { administration; good } \\
\text { inter-rater reliability. }\end{array}$ & $\begin{array}{l}\text { Requires further study to } \\
\text { explore convergent validity } \\
\text { with other established } \\
\text { measures; test retest reliability } \\
\text { is unknown. }\end{array}$ \\
\hline $\begin{array}{l}\text { Separation Anxiety } \\
\text { Test } \\
\text { (SAT) }\end{array}$ & $\begin{array}{l}9 \\
\text { photographs }\end{array}$ & $\begin{array}{l}\text { Attachment }(5-20) \\
\text { Self-reliance }(4-16) \\
\text { Avoidance }(9-27)\end{array}$ & $?$ & $\begin{array}{l}3 \text { dimensions: } \\
\text { attachment, self-reliance, } \\
\text { avoidance }\end{array}$ & $\begin{array}{l}\text { Generalized } \\
\text { attachment }\end{array}$ & $\begin{array}{l}\text { Suitable for younger } \\
\text { end of middle } \\
\text { childhood. }\end{array}$ & $\begin{array}{l}\text { Psychometric properties } \\
\text { generally inadequate. }\end{array}$ \\
\hline
\end{tabular}


Table 4.2 Characteristics, strengths and weaknesses of self-report measures

\begin{tabular}{|c|c|c|c|c|c|c|c|}
\hline Measure & \# Items & Scales & $\begin{array}{l}\text { Response } \\
\text { options }\end{array}$ & Range of scores & $\begin{array}{l}\text { Focus of } \\
\text { measure }\end{array}$ & Strengths & Weaknesses \\
\hline$\overline{\mathrm{AAQ}}$ & 9 & $\begin{array}{l}\text { Angry distress } \\
\text { Availability } \\
\text { Goal-corrected } \\
\text { partnership }\end{array}$ & $1-5$ & $?$ & Parents & $\begin{array}{l}\text { Brief measure, } \\
\text { adequate structural } \\
\text { validity. }\end{array}$ & $\begin{array}{l}\text { Inadequate internal } \\
\text { consistency; doubtful } \\
\text { content validity. }\end{array}$ \\
\hline AFAS & 30 & $\begin{array}{l}\text { Secure } \\
\text { Anxious } \\
\text { Avoidant }\end{array}$ & $1-5$ & $1-5$ & Peers & $\begin{array}{l}\text { Adequate internal } \\
\text { consistency, test } \\
\text { retest reliability, } \\
\text { content validity. }\end{array}$ & $\begin{array}{l}\text { Inadequate structural } \\
\text { validity. }\end{array}$ \\
\hline AFAS-SF & 15 & $\begin{array}{l}\text { Secure } \\
\text { Anxious } \\
\text { Avoidant }\end{array}$ & $1-5$ & $1-5$ & Peers & $\begin{array}{l}\text { Brief, adequate } \\
\text { internal consistency. }\end{array}$ & $\begin{array}{l}\text { Inadequate structural } \\
\text { validity. }\end{array}$ \\
\hline ASQ & 40 & $\begin{array}{l}\text { Confidence; Relationships } \\
\text { as secondary; Discomfort } \\
\text { with closeness; } \\
\text { Preoccupation with } \\
\text { relationships; Need for } \\
\text { approval; }\end{array}$ & $1-6$ & $40-240$ & Generalized & $\begin{array}{l}\text { Adequate internal } \\
\text { consistency; } \\
\text { applicable to both } \\
\text { adolescents and } \\
\text { adults; widely used } \\
\text { in adult studies. }\end{array}$ & $\begin{array}{l}\text { Inadequate structural } \\
\text { validity; few } \\
\text { psychometric } \\
\text { properties evaluated in } \\
\text { adolescent samples. }\end{array}$ \\
\hline AUAQ & 9 & $\begin{array}{l}\text { Failed protection } \\
\text { Anger/dysregulation } \\
\text { Fear }\end{array}$ & $1-5$ & $?$ & Parents & $\begin{array}{l}\text { Adequate content } \\
\text { and structural } \\
\text { validity. }\end{array}$ & $\begin{array}{l}\text { Inadequate internal } \\
\text { consistency. }\end{array}$ \\
\hline ECR-RC & 36 & $\begin{array}{l}\text { Avoidance } \\
\text { Anxiety }\end{array}$ & $1-7$ & $?$ & Parent & $\begin{array}{l}\text { Adequate internal } \\
\text { consistency and } \\
\text { hypothesis testing; } \\
\text { based on widely } \\
\text { used adult } \\
\text { attachment style } \\
\text { measure. }\end{array}$ & $\begin{array}{l}\text { Inadequate structural } \\
\text { validity. }\end{array}$ \\
\hline ECR-RS & 9 & $\begin{array}{l}\text { Avoidance } \\
\text { Anxiety }\end{array}$ & $1-7$ & $\begin{array}{l}6-42 \text { (Avoidance) } \\
3-21 \text { (Anxiety) }\end{array}$ & $\begin{array}{l}\text { Parent, best } \\
\text { friend, romantic } \\
\text { partner }\end{array}$ & $\begin{array}{l}\text { Adequate internal } \\
\text { consistency; based } \\
\text { on widely used adult } \\
\text { attachment style } \\
\text { measure. }\end{array}$ & $\begin{array}{l}\text { Inadequate hypothesis } \\
\text { testing; uncertain } \\
\text { structural validity. }\end{array}$ \\
\hline
\end{tabular}


Table 4.2 Characteristics, strengths and weaknesses of self-report measures (continued)

\begin{tabular}{|c|c|c|c|c|c|c|c|}
\hline Measure & \# Items & Scales & $\begin{array}{l}\text { Response } \\
\text { options }\end{array}$ & Range of scores & $\begin{array}{l}\text { Focus of } \\
\text { measure }\end{array}$ & Strengths & Weaknesses \\
\hline ECR-R- GSF & 20 & $\begin{array}{l}\text { Avoidance } \\
\text { Anxiety }\end{array}$ & $1-5$ & $1-5$ & Generalized & $\begin{array}{l}\text { Adequate content } \\
\text { validity and internal } \\
\text { consistency; based } \\
\text { on widely used adult } \\
\text { attachment style } \\
\text { measure. }\end{array}$ & $\begin{array}{l}\text { Inadequate hypothesis } \\
\text { testing and structural } \\
\text { validity. }\end{array}$ \\
\hline IPPA & $\begin{array}{l}81(28 \# \text { each } \\
\text { parent, } \\
25 \# \text { peers })\end{array}$ & $\begin{array}{l}\text { Communication } \\
\text { Trust } \\
\text { Alienation }\end{array}$ & $1-5$ & $\begin{array}{l}\text { 16-92 (Mother, } \\
\text { Father) } \\
19-82 \text { (Peer) }\end{array}$ & $\begin{array}{l}\text { Mother, father, } \\
\text { peers }\end{array}$ & $\begin{array}{l}\text { Studied relatively } \\
\text { extensively across a } \\
\text { range of samples and } \\
\text { countries; adequate } \\
\text { internal consistency } \\
\text { and hypothesis } \\
\text { testing. }\end{array}$ & $\begin{array}{l}\text { Lengthy; inadequate } \\
\text { structural validity. }\end{array}$ \\
\hline IPPA-B & $\begin{array}{l}28 \#(14 \# \\
\text { mother, } 14 \# \\
\text { father) }\end{array}$ & $\begin{array}{l}\text { Communication } \\
\text { Trust } \\
\text { Alienation }\end{array}$ & $1-5$ & $?$ & Parent & $\begin{array}{l}\text { Brief version of a } \\
\text { widely-studied } \\
\text { measure; adequate } \\
\text { internal consistency, } \\
\text { structural validity } \\
\text { and hypothesis } \\
\text { testing. }\end{array}$ & $\begin{array}{l}\text { Only validated in one } \\
\text { study of fair quality; } \\
\text { only available in } \\
\text { French. }\end{array}$ \\
\hline IPPA-45 & $\begin{array}{l}45 \text { (15\# each } \\
\text { for mother, } \\
\text { father, peers) }\end{array}$ & $\begin{array}{l}\text { Communication } \\
\text { Trust } \\
\text { Alienation }\end{array}$ & $1-5$ & $?$ & $\begin{array}{l}\text { Mother, father, } \\
\text { peers }\end{array}$ & $\begin{array}{l}\text { Adequate internal } \\
\text { consistency; shorter } \\
\text { version of a widely- } \\
\text { studied measure. }\end{array}$ & $\begin{array}{l}\text { Inadequate structural } \\
\text { validity. }\end{array}$ \\
\hline IPPA-R & $\begin{array}{l}81(28 \# \text { each } \\
\text { parent, } \\
25 \# \text { peers })\end{array}$ & $\begin{array}{l}\text { Communication } \\
\text { Trust } \\
\text { Alienation }\end{array}$ & & $\begin{array}{l}\text { 16-92 (Mother, } \\
\text { Father) } \\
19-82 \text { (Peer) }\end{array}$ & $\begin{array}{l}\text { Mother, father, } \\
\text { peers }\end{array}$ & $\begin{array}{l}\text { Adequate } \\
\text { hypothesis-testing } \\
\text { and content validity. }\end{array}$ & $\begin{array}{l}\text { Lengthy; inadequate } \\
\text { internal consistency } \\
\text { and structural validity. }\end{array}$ \\
\hline IPPA-S & $\begin{array}{l}24 \text { (12\# parent, } \\
12 \# \text { peer })\end{array}$ & $\begin{array}{l}\text { Communication } \\
\text { Trust } \\
\text { Alienation }\end{array}$ & $1-5$ & $?$ & Parent, peers & $\begin{array}{l}\text { Brief version of a } \\
\text { widely-studied } \\
\text { measure. }\end{array}$ & $\begin{array}{l}\text { Inadequate internal } \\
\text { consistency, structural } \\
\text { validity and hypothesis } \\
\text { testing. }\end{array}$ \\
\hline
\end{tabular}


Table 4.2 Characteristics, strengths and weaknesses of self-report measures (continued)

\begin{tabular}{|c|c|c|c|c|c|c|c|}
\hline Measure & \# Items & Scales & $\begin{array}{l}\text { Response } \\
\text { options }\end{array}$ & Range of scores & $\begin{array}{l}\text { Focus of } \\
\text { measure }\end{array}$ & Strengths & Weaknesses \\
\hline$\overline{P A C Q}$ & 36 & $\begin{array}{l}\text { Avoidant } \\
\text { Preoccupied }\end{array}$ & $0-2$ & $\begin{array}{l}0-36 \text { (Avoidant) } \\
0-32 \text { (Preoccupied) }\end{array}$ & Parent & $\begin{array}{l}\text { Adequate internal } \\
\text { consistency. }\end{array}$ & $\begin{array}{l}\text { Inadequate content } \\
\text { validity and hypothesis } \\
\text { testing. }\end{array}$ \\
\hline PIML & $\begin{array}{l}44 \text { ( } 20 \# \text { parent, } \\
24 \# \text { peer) }\end{array}$ & $\begin{array}{l}\text { Communication } \\
\text { Trust } \\
\text { Alienation }\end{array}$ & $1-5$ & $?$ & Parent, peers & $\begin{array}{l}\text { Based on widely- } \\
\text { studied IPPA; } \\
\text { adequate hypothesis } \\
\text { testing and structural } \\
\text { validity. }\end{array}$ & $\begin{array}{l}\text { Inadequate internal } \\
\text { consistency. }\end{array}$ \\
\hline SS & 15 & Security & $1-4$ & $1-4$ & Parent & $\begin{array}{l}\text { Brief measure } \\
\text { developed for } \\
\text { middle childhood. }\end{array}$ & $\begin{array}{l}\text { Few psychometric } \\
\text { properties evaluated; } \\
\text { conflicting findings } \\
\text { about adequacy of } \\
\text { internal consistency } \\
\text { and hypothesis testing. }\end{array}$ \\
\hline
\end{tabular}


Table 4.3 COSMIN Criteria for adequacy of measurement properties

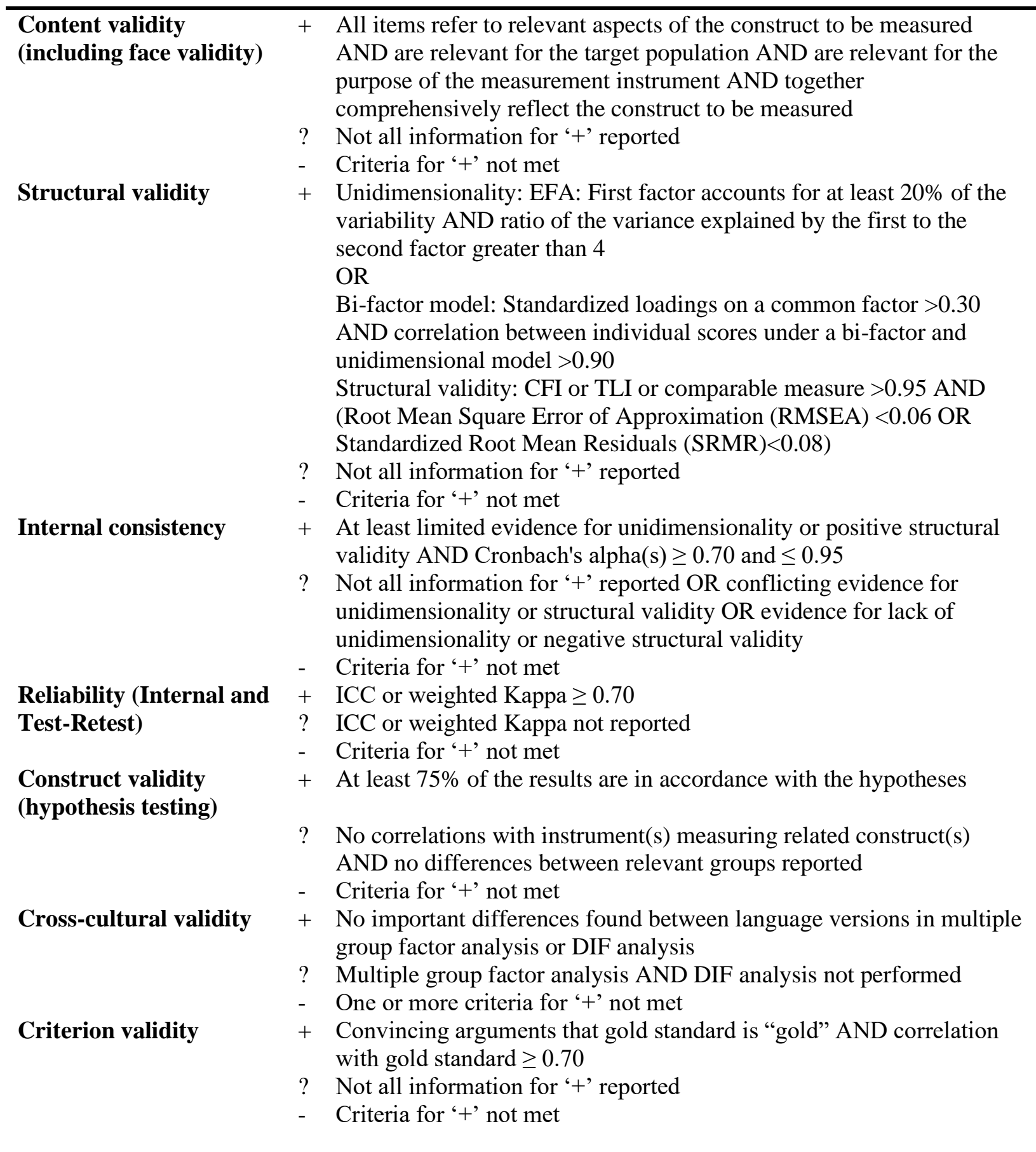


Table 4.4 Synthesis of psychometric properties and level of evidence for observer-rated measures

\begin{tabular}{|c|c|c|c|c|c|c|c|c|}
\hline Measure & $\begin{array}{l}\text { Internal } \\
\text { consistency }\end{array}$ & $\begin{array}{l}\text { Test retest } \\
\text { reliability }\end{array}$ & $\begin{array}{l}\text { Inter-rater } \\
\text { reliability }\end{array}$ & $\begin{array}{l}\text { Content } \\
\text { validity }\end{array}$ & $\begin{array}{l}\text { Structural } \\
\text { validity }\end{array}$ & $\begin{array}{l}\text { Hypothesis } \\
\text { testing }\end{array}$ & $\begin{array}{l}\text { Cross-cultural } \\
\text { validity }\end{array}$ & $\begin{array}{l}\text { Criterion } \\
\text { validity }\end{array}$ \\
\hline$\overline{\mathrm{AAI}}$ & & & $?$ & & & + & & \\
\hline AAP & & & ++ & & & + & & \\
\hline AAPQ & & & & & & - & & \\
\hline ASCT & $?$ & & --- & & $?$ & -- & & \\
\hline ASA & $?$ & & ++ & & ++ & $+1-$ & & \\
\hline BND & & & ++ & & & - & & \\
\hline CAI & $?$ & -- & $+1-$ & +++ & +++ & $+1-$ & & \\
\hline CMSSB & & & $?$ & & & + & & \\
\hline CMCAST & & & - & + & & & & - \\
\hline GPACS & & & ++ & & $?$ & -- & & \\
\hline MCAST & - & $?$ & -- & + & $?$ & - & $?$ & \\
\hline SBST & $?$ & & ++ & & & -- & & \\
\hline SAT & $?$ & $?$ & $?$ & $?$ & & $+/-$ & & \\
\hline
\end{tabular}


Table 4.5 Synthesis of psychometric properties and level of evidence for self-report measures

\begin{tabular}{|c|c|c|c|c|c|c|c|}
\hline $\begin{array}{l}\text { Name of } \\
\text { measure }\end{array}$ & $\begin{array}{l}\text { Internal } \\
\text { consistency }\end{array}$ & $\begin{array}{l}\text { Test retest } \\
\text { Reliability }\end{array}$ & $\begin{array}{l}\text { Content } \\
\text { validity }\end{array}$ & $\begin{array}{l}\text { Structural } \\
\text { validity }\end{array}$ & $\begin{array}{l}\text { Hypothesis } \\
\text { testing }\end{array}$ & $\begin{array}{l}\text { Cross - cultural } \\
\text { validity }\end{array}$ & $\begin{array}{l}\text { Criterion } \\
\text { validity }\end{array}$ \\
\hline AAQ & --- & - & - & +++ & - & $?$ & \\
\hline AFAS & +++ & $?$ & ++ & --- & -- & & \\
\hline AFAS - SF & + & & & - & + & & + \\
\hline AUAQ & --- & $?$ & + & +++ & - & $?$ & \\
\hline ASQ & +++ & & & --- & & & \\
\hline $\mathrm{ECR}-\mathrm{RC}$ & + & & & - & + & & \\
\hline $\mathrm{ECR}-\mathrm{RS}$ & +++ & & & $?$ & - & & \\
\hline $\mathrm{ECR}-\mathrm{R}-\mathrm{GSF}$ & + & & ++ & - & - & & \\
\hline IPPA & $+/-$ & & ++ & - & ++ & $?$ & \\
\hline IPPA -45 & +++ & & & --- & & & \\
\hline IPPA $-\mathrm{R}$ & -- & & & - & ++ & & \\
\hline IPPA $-S$ & - & & & - & - & & \\
\hline IPPA - B & + & & & + & + & $?$ & \\
\hline PIML & - & & & + & + & & \\
\hline PACQ & $?$ & $?$ & - & & -- & & \\
\hline SS & $?$ & & & & $+/-$ & & \\
\hline
\end{tabular}

Note. +++ or ---, strong evidence; ++ or --, moderate evidence; + or -, limited evidence; +/ - , conflicting findings; ?, unknown; blank cell, no evidence available. 


\subsection{Results}

Our search yielded 601 articles once duplicates were removed (see Figure 4.1). TG and KW separately screened by title and abstract, then assessed eligibility in 101 fulltext articles. Disagreements at screening stage were resolved by TJ. Reference lists were checked for additional articles not picked up in the search stage. Fifty-three relevant articles were identified (see Appendix F for references of included studies).

\section{$\underline{4.4 .1 \text { Internal consistency }}$}

Adequate internal consistency (alpha > 0.7) was reported in studies of the following self-report measures: AFAS, AFAS-SF, ASQ, ECR-RC, ECR-RS, ECR-R-GSF, IPPA-B and IPPA-45. There were studies reporting both adequate and inadequate internal consistency for the SS, PACQ and IPPA. Study quality was generally fair, but was excellent in the case of the AFAS, AFAS-SF, ASQ, ECR-RS and IPPA-45.

For observer-rated measures, several studies examined internal consistency across questions and sub-scales (ASA, ASCT, CAI, FFI, SAT, SBST). Only the AQ-A and ASA reported adequate internal consistency, both in studies of poor methodological quality for the evaluation of this property.

\section{$\underline{4.4 .2}$ Test-retest reliability}

Test-retest reliability data was notably lacking for most measures. It was reported for the AAQ, AFAS, AUAQ, PACQ, CAI and MCAST but no studies met COSMIN adequacy criteria (ICC or kappa > 0.7). 
Figure 4.1 Flowchart for search strategy

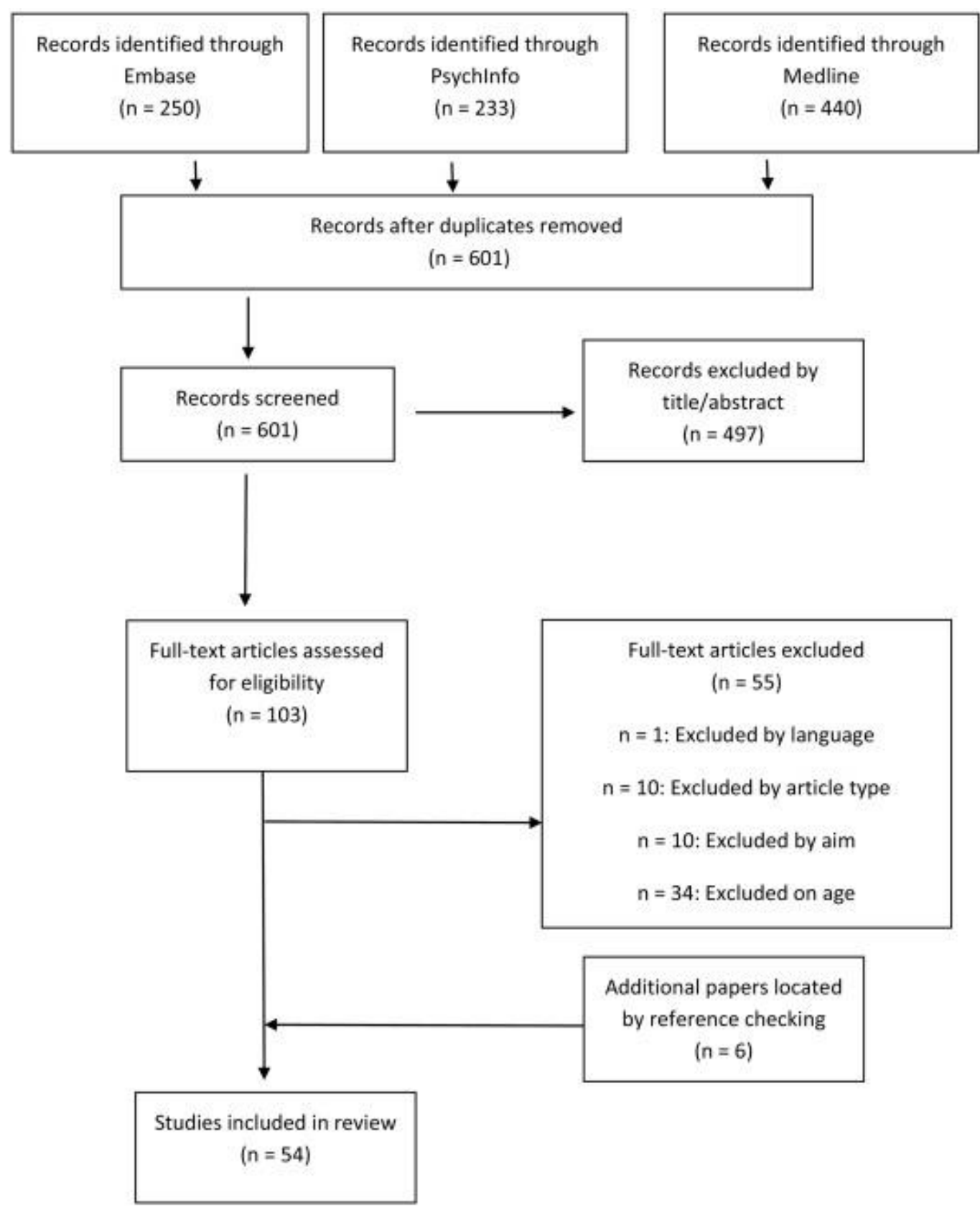




\subsubsection{Inter-rater reliability (interview and projective measures)}

Adequate inter-rater reliability was reported for the AAI, AAP, BND, CAI and two

measures using the secure base script paradigm, the ASA and SBST. The CAI demonstrated good inter-rater reliability in the study by Borelli et al. (2016a), but not in earlier studies.

\section{$\underline{\text { 4.4.4 Content validity }}$}

Content validity proved hard to assess using the standard COSMIN criteria, thus these were adapted. Studies were rated as excellent for methodological quality if (1) they demonstrated evidence of iterations in the development of the measure, (2) if the assumptions underlying the measure were tested (e.g. through piloting), and (3) if they involved an expert panel. Face validity of the measure in terms of its theoretical links to attachment theory were also considered. Positive ratings of content validity were given to the AFAS, CAI, ECR-R-GSF and IPPA in studies of good or excellent methodological quality, and also to the AUAQ, CMCAST, MCAST, SAA and SAT in studies of fair or poor methodological quality.

\section{$\underline{\text { 4.4.5 Structural validity }}$}

Several studies evaluated factor structure using exploratory and confirmatory factor analysis. Adequate structural validity was reported for the AAQ, AUAQ, IPPA-B and PIML. 
For observer-rated measures, factor analyses were conducted, typically using subscales in the place of items. In most such studies the results were given an indeterminate rating ('?’) since the findings were not interpretable within the COSMIN scheme. These indeterminate ratings were given to structural validity studies of the ASCT, MCAST and CAI, with the exception of the Zachrisson et al. (2011) study of the CAI, which was given a positive rating for adequate measurement properties and rated as excellent for methodological quality. This study is notable in that the confirmatory factor analysis demonstrated adequate model fit, as defined by COSMIN, for a two-factor model comprised of avoidance and preoccupation. One other notable study of structural validity was that by Waters et al. (2015). This was a taxometric study pointing to the dimensional structure of attachment as measured by the Attachment Script Assessment.

\subsubsection{Hypotheses testing}

Under the COSMIN scheme, various aspects of construct validity, such as convergent and discriminant validity, are assessed under the banner of 'hypotheses testing'. More favourable ratings of methodological quality are assigned for studies that test multiple, specific hypotheses including the direction and magnitude of correlations. Of the observer-rated measures, ratings of adequate construct validity were assigned to studies of the AAI, AAP and CMSSB. The ASA, CAI, SAA and SAT showed inconsistent findings, with both positive and negative studies of hypotheses testing reported. For self-report measures, adequate ratings were given to studies of the AFAS-SF, ECR-RC, IPPA, IPPA-B, IPPA-R and PIML. Findings for the SS were inconsistent. 


\section{$\underline{4.4 .7 \text { Cross-cultural validity }}$}

Few studies specifically investigated cross-cultural validity as defined within the COSMIN taxonomy, which refers to the degree to which the performance of a translated or culturally-adapted instrument is an adequate reflection of its performance in its original version. Where it was investigated, study quality was rated as poor. Adequate cross-cultural validity was demonstrated for the FFI. An indeterminate rating was given in studies of the AAQ, AUAQ, IPPA, IPPA-B, and MCAST.

\section{$\underline{4.4 .8}$ Criterion validity}

Studies of criterion validity were rare, which is unsurprising in a field lacking an accepted 'gold standard' measure. The AFAS-SF demonstrated adequate criterion validity against the AFAS, whilst the CMCAST did not do so against the MCAST.

\subsubsection{Synthesis of results}

Our synthesis of the strength of the evidence for psychometric properties is presented in Tables 4.4 and 4.5 (observer-rated and self-report, respectively). Overall, no measure has demonstrated consistent evidence of good psychometric properties across a range of criteria. However, our findings point to the CAI and IPPA currently having the best evidence of adequate measurement properties. The CAI has positive findings in support of its content validity, structural validity when assessed using two dimensions (Zachrisson et al., 2010), and various positive findings relating to construct validity (e.g. Borelli, 2016). However, its inter-rater reliability is suboptimal in most studies, with the exception of that by Borelli (2016). The IPPA exists in several versions, none of which have emerged as demonstrating adequacy across a 
range of psychometric properties. In general, the structural validity of the measure is inadequate, and findings on internal consistency have been mixed. However, it has demonstrated adequate construct validity across a relatively large number of studies compared to other self-report measures.

\subsection{Discussion}

Overall our review points to a lack of evidence of adequate measurement properties for most available attachment measures in middle childhood. However, we wish to draw attention to some important points that should be borne in mind when interpreting our findings.

Firstly, the COSMIN tool yields categories, with necessarily arbitrary values chosen as cut-offs to distinguish adequate from inadequate measurement properties. In some cases the statistical values that led to a negative rating were close to the value required for a positive rating. This point applies similarly to the ratings of methodological quality, in which COSMIN operates a 'worst score counts' algorithm. This means that the final rating of methodological quality is defined by the lowest score obtained for that measurement property; thus a single flaw could lead to a rating of 'fair' when it would otherwise have been rated 'excellent'. We applied a similar rule in rating the adequacy of a measurement property where data for several subscales were presented: one sub-optimal value was enough to lead to a negative rating of adequacy for that property. 
The implication of all these points is that a quick glance at our ratings may lead to an underestimation of both the adequacy of measurement properties and also the methodological quality of the evidence. Finally, readers should note that we followed the COSMIN guidance around study selection, including only studies that specifically stated the investigation of measurement properties as an aim of the study (De Vet et al., 2011). Previous reviews (e.g. Kerns and Brumariu, 2016) have included a broader range of studies as providing evidence of validity, such as studies looking at associations between attachment and emotional regulation (e.g. Brumariu, Kerns and Siebert, 2011). However, including studies that did not specifically aim to examine psychometric properties would have increased the risk of bias, led to an unwieldy number of studies for review, and made it harder for future researchers to reproduce our review. Nevertheless, we accept that by using COSMIN we have taken a relatively stringent approach to the selection and rating of studies.

We believe that this review has helpfully summarised the state of evidence on psychometric properties for individual measures. In addition, this review also allows us to consider some broader questions that we consider to be fundamental to the measurement and conceptualisation of attachment in middle childhood and adolescence. We have organised our discussion around two key questions, before moving on to recommendations for research and clinical practice. 
4.5.1 What is the most valid and reliable approach to assessing attachment in middle childhood and adolescence?

In general we do not consider, a priori, that any measurement approach is inherently more appropriate to the measurement of attachment across middle childhood and adolescence. One exception to this is the assessment of attachment in the early phase of middle childhood (age 6-8 years) in which self-report measures are unlikely to be valid as a consequence of children's more limited reading and cognitive ability. Unfortunately, our review points to the relatively poor psychometric properties of measures developed for this age group (e.g. Green et al., 2000). The CAI was initially developed for children aged 7-13 years, and currently has the best evidence of psychometric properties for early middle childhood. We believe a study of psychometric properties of the CAI in a sample of 6-9 year-olds would be worthwhile, as currently there is a lack of evidence for measures demonstrating adequate psychometric properties for this age group.

In older middle childhood and adolescence, interviews constitute a well-validated measurement approach. Whilst the CAI has been studied in adolescence (Venta et al., 2014) clinicians and researchers may want to consider whether the AAI or AAP might be more appropriate for older teenagers.

We note that measures of secure base scriptedness (Dykas et al., 2006; Psouni et al., 2014), in which the task involves creating stories using word prompts, have demonstrated relatively strong evidence of adequate psychometric properties. Such measures have numerous advantages including shorter administration time and 
simpler scoring method when compared to interview measures such as the CAI. Further studies examining convergent validity of scriptedness measures with the bestperforming interview and self-report measures would be an important contribution to the field.

Finally, the reliability, and especially validity, of self-report measures of attachment is important to consider, not least since NICE (2015) did not include such measures in their guideline on attachment. Most self-report measures in this review did not examine convergent validity with interview or projective assessment methods. Two exceptions are the IPPA and SS, which have both been found to be correlated with attachment as measured by the CAI (Borelli et al, 2016). Importantly, however, the correlations were below the 0.4 cut-off which is used by convention as evidence that two measures are tapping the same construct. Thus, strictly speaking, we can conclude that some self-report measures of attachment are correlated with attachment interviews, but we cannot be certain they are rating the same construct. For adolescents, measures such as the ECR and ASQ can be used to assess attachment styles, which can be assumed to have conceptual continuity with the attachment style construct as measured in adults. Such measures are needed in order to shed light on the developmental antecedents of adult attachment styles, for instance through longitudinal studies (e.g. Jones et al., 2018). However, as we discuss, there is scope for the improvement of self-report measures. 


\subsubsection{Is attachment distributed categorically or continuously?}

The four-category ABCD paradigm has held a central place in attachment theory and measurement approaches. In our review, interview and projective measures based on this paradigm typically reported sub-optimal structural validity and inter-rater reliability (kappa < 0.7). By contrast, dimensional approaches to scoring such measures demonstrated favourable reliability (e.g. Psouni, 2014; Waters et al., 2015). Importantly, Zachrisson et al. (2011) found evidence of a factor structure comprising two dimensions underlying the CAI. Thus the findings from this review appear to converge with emerging findings at other points in the lifespan, with taxometric analyses of both the Strange Situation Procedure (Fraley and Spieker, 2003) and the Adult Attachment Interview (Fraley and Roisman, 2014) supporting the idea of attachment being distributed across two dimensions, rather than four categories.

If attachment is distributed continuously, are self-report measures yielding continuous scales the best approach to measuring it? Unfortunately, almost all self-report measures in this review demonstrated sub-optimal structural validity. This review included various self-report measures, often based on adult attachment style measures. These measures have often been subject to numerous revisions, such as changes to wording and item length (e.g. ECR and IPPA studies). Despite exhaustive factor analysis using large samples, these measures have failed to meet criteria for good structural validity as defined by COSMIN. This raises difficult questions for the field. Does the lack of structural validity reflect problems with the measures themselves, or are the constructs they set out to measure not reflective of the phenomenology of attachment in middle childhood and adolescence? Likewise, does attachment in this age group not break down into the $\mathrm{ABCD}$ categories, or are available measures not 
able to detect them reliably? Based on the evidence in this review, it seems plausible that the attachment construct in middle childhood and adolescence is inherently difficult to measure reliably. This may be because attachment representations themselves are relatively fluid at this age (Jones et al., 2018); perhaps also because the developing nature of children's cognitive and socio-emotional abilities presents challenges in capturing such a complex construct.

Thus, the underlying structure of attachment in middle childhood and adolescence is unclear, with neither the ABCD model for interview/projective measures, nor the two factor (avoidance/anxiety) structure of adult attachment style measures demonstrating strong evidence of validity in this age group. This has important implications for research and clinical practice.

\section{$\underline{4.5 .3 \text { Implications for research }}$}

In keeping with the findings by NICE (2015), our study highlights the relatively poor methodological quality of many studies in the field. However, within our review we note with encouragement a trend towards improved study quality over time. Some key methodological principles worth highlighting are: clearly stated hypotheses that include predictions about the direction and magnitude of expected correlations, and reporting on both the amount and handling of missing data and explaining how missing data is handled. Studies of test-retest reliability and sensitivity to change are required, as are studies investigating attachment measures in a range of different sociocultural contexts. Recent studies conducted in Africa and Asia (Sochos \& Lokshum, 2017; 
Wan et al., 2017) are a welcome development for the field; more such studies are needed.

For interview measures based on the ABCD paradigm (e.g. CAI, MCAST), research on simpler coding systems yielding dimensional scores of avoidance and preoccupation, would lead to a number of benefits. These include improved inter-rater reliability, theoretical congruence with other developments in the field (Roisman, 2014; Roisman and Shaver 2018), and increased statistical power in research, such as longitudinal designs investigating the impact of attachment on developmental outcomes (Wright et al., 2018).

Importantly, research is needed that sheds light on the underlying structure of attachment in middle childhood and adolescence. Structural equation modelling (SEM) could be used to investigate the extent to which different measures, and indeed individual items, load on to a latent attachment variable. Ideally this would be undertaken with large datasets in which a variety of measurement approaches have been taken (i.e. interview, projective, self-report), including measures of attachment and associated constructs such as mentalizing and emotion regulation. Exploratory work of this kind should be possible with existing data sets, although the ideal would be to design studies with large samples that could simultaneously investigate the structure of attachment and evaluate new measures. This should include the development of new self-report measures, the starting point for which must be a clear conceptualisation of what is meant by attachment. In studies in which attachment has been measured by a variety of methods, the process of refining the questionnaire 
should include the extent to which items load on to a latent attachment variable in SEM. By this means, the measure would avoid the pitfalls of shared method variance and reliance on adult attachment style models for concurrent validity.

Our review also highlights a clear need for research on measurement error. Fearon and Roisman (2017) recommend the development of better attachment measures that allow direct assessment of the relationship between indicators, error and underlying constructs. Once again, studies employing structural equation modelling could investigate the degree of measurement error for each item in a self-report questionnaire, or each scale in an interview/projective measure, by assessing correlations with a latent attachment variable.

\subsubsection{Implications for clinical practice}

Attachment theory is an influential theoretical framework in many clinical contexts such as psychological therapies with children and families. In the UK, national guidelines published by NICE (2015) specifically advise clinicians working with children in child protection and adoption settings to consider attachment in their assessment and treatment planning, and recommend the MCAST, CAI and AAI. Our findings suggest that such measures are vulnerable to high measurement error, as evidenced by unfavourable ratings of inter-rater reliability in many studies. We therefore suggest that, when used, the findings of such measures should be interpreted tentatively as a clinical hypothesis, and understood as being informative only as one aspect of a much broader assessment of a child and their caregivers; moreover, this hypothesis may need to be reviewed over time as new information emerges. In court 
settings, evidence provided from such measures that has been rated by only one clinician should not be seen as authoritative, since a second rater may well disagree on the assignment of attachment category. Finally, we encourage clinicians, policymakers and members of the public not to reify attachment categories. Studies in this review suggest that attachment status is not necessarily predictive of psychopathology in children and adolescents. For instance, in studies using the CAI, rates of secure attachment in clinical samples have been as high as $30 \%$ in adolescent psychiatric inpatients (Venta et al., 2014), whilst Scott et al. (2011) reported security in 52\% and $73 \%$ respectively in moderate and high-risk samples for conduct problems. This compares with rates of approximately $60 \%$ attachment security in normative child samples (Shmueli-Goetz et al., 2008; Green et al., 2000).

\section{$\underline{4.5 .5 \text { Limitations }}$}

As discussed earlier, our review applied stringent inclusion criteria. As a consequence, our review may under-report the breadth of evidence on psychometric properties for attachment measures in middle childhood and adolescence, although excluded studies are likely to be of low-quality. On the other hand, we were not able to appraise the risk of bias arising from publication bias across studies. Studies in our review reported a range of different statistics across a variety of measurement properties; as such, there was no valid and reliable way to assess publication bias. Given the likely researcher bias towards publishing positive results, it is possible that our review over-estimates the adequacy of psychometric properties across measures, since there may be unpublished data showing negative findings. 


\subsection{Conclusion}

The field of attachment is entering an exciting phase in which new empirical and theoretical insights are emerging. Longitudinal studies of attachment stability suggest that attachment may be less stable in adolescence than in adulthood (Jones et al., 2018), and that genetic influences on attachment may come in to play in adolescence that were not present in infancy (Fearon et al., 2014). Recent theories have suggested that the early stages of sexual maturation (andrenarche) may constitute a 'switchpoint' in the development of attachment strategies (Del Giudice, 2009), and that adult attachment styles may be more influenced by recent interpersonal experiences than distal, early caregiving experiences (Fraley and Roisman, 2018). The attachment field is reliant on the availability of valid, reliable and sensitive measures that can be used to test theories and build evidence. In the clinical realm, good measures are needed to test both aetiological models in which childhood attachment experiences are implicated (e.g. Fonagy et al., 2002) and also the role of attachment in treatments (e.g. Diamond et al., 2010). For clinicians working in areas such as adoption, fostering and child protection, the availability of good attachment measures would be of enormous utility.

Selecting a suitable attachment measure, whether in a clinical or research context, is a complex matter. Our review provides various important sources of information that can guide the decision including measurement approach (i.e. interview, task, selfreport), administration time, and the type of attachment relationship that is assessed. In the continuing absence of any measure that can be considered a 'gold standard', we advise close scrutiny of face validity: that is, clinicians and researchers need to assess 
the extent to which the conceptualisation of attachment and how it is assessed fit with the purposes for which the measure is being chosen.

Our review suggests that there are currently large gaps in our knowledge of the psychometric properties of attachment measures, with the lack of data on sensitivity to change being particularly regrettable. We found limited evidence of adequate psychometric properties, but identified the CAI and IPPA as currently having the best evidence of such properties amongst observer-rated and self-report measures respectively. The ASA, a measure of secure base scriptedness, was identified as a promising measure worthy of future research on psychometric properties. Our findings point to the advantages of dimensional rather than categorical approaches to measurement, with more favourable inter-rater reliability and structural validity ratings observed in measures yielding dimensional scores. Future studies are needed that test specific hypotheses and that shed light on the underlying structure of attachment representations in middle childhood and adolescence. 
Chapter 5 - Attachment and mentalization as predictors of outcome in family therapy for adolescent anorexia nervosa

This chapter is an extended version of a manuscript currently under review and is available as a preprint with the following details:

Jewell, T., Herle, M., Serpell, L., Eivors, A., Simic, M., Fonagy, P., \& Eisler, I. (2020, November 9). Attachment and mentalization as predictors of outcome in family therapy for adolescent anorexia nervosa. https://doi.org/10.31234/osf.io/fgytk 


\subsection{Abstract}

Objective: Anorexia nervosa focussed family therapy (FT-AN) is the first-line treatment for adolescent anorexia nervosa (AN), but the predictors of poor treatment response are not well-understood. The main aim of this study was to investigate the role of attachment and mentalization in predicting treatment outcome at nine months. Therapeutic alliance at one month was tested as a potential mediator of the effect of attachment and mentalization at baseline on outcome at nine months.

Method: 192 adolescents with AN and their parents were recruited as they began family therapy in out-patient specialist eating disorder services. Self-report measures of attachment, mentalization and emotion regulation were completed at the start of treatment by adolescent patients and one of their parents. Self-reported alliance scores were collected at one month.

Results: Higher scores on the Certainty Scale of the Reflective Functioning Questionnaire, completed by parents, which indicate over-certainty about mental states, were the strongest predictor of poor outcome (Odds Ratio: 0.42 , CI: 0.20 to 0.87, $\mathrm{p}=0.02$ ). Similarly, for adolescents, higher Lack of Clarity scores on the Difficulties in Emotion Regulation Scale, representing being unclear about one's feelings, were predictive of positive treatment outcome (OR: 1.10, CI: $1.00-1.21$, $\mathrm{p}=0.04$ ). Alliance scores at one month were predictive of outcome but did not play a mediating role in relation to baseline predictors.

Conclusion: These novel findings suggest that, particularly in parents, a tendency towards inappropriate certainty about mental states in others may predict poor outcome in family therapy for adolescent AN. Further research is warranted to replicate the finding and characterise families at risk of poor outcome. 


\subsection{Introduction}

Anorexia nervosa (AN) is a serious condition which typically emerges during adolescence. AN is associated with high rates of psychiatric co-morbidity (SalbachAndrae et al., 2008) and significantly raised mortality rates (Arcelus et al., 2011), with an estimated incidence of 14 per 100,000 (Petkova et al., 2019). Specialist anorexia nervosa focused family therapy for children and young people (FT-AN) has the strongest efficacy evidence in the treatment of adolescent AN and was recommended by the National Institute for Health and Care Excellence (2017) as the first-line treatment. However, a significant proportion of patients fail to respond and a lack of evidence on predictors of treatment response and understanding of mechanisms of change (Kazdin, 2007) limits efforts to personalize treatment recommendations. There is therefore an urgent need for well-designed and sufficiently powered research that can investigate predictors of response and change processes in FT-AN (Hamadi \& Holliday, 2020).

Currently, the most robust findings relating to treatment response in FT-AN relate to clinical indicators, with shorter duration of illness and higher percentage body mass index at start of treatment being associated with more positive outcomes (see Chapter 2 (Jewell et al., 2016a)). In terms of early markers, early weight gain (Doyle et al., 2010; Hughes et al., 2019a) and positive ratings of the therapeutic alliance are predictive of good treatment response in FT-AN (Isserlin \& Couturier, 2012) although alliance and outcome are likely to have a reciprocal relationship (see meta-analysis by (Graves et al., 2017a)). In terms of relational variables, there is evidence that parental expressed emotion is associated with poorer outcome (see Simic, Jewell and Eisler, 2020 for a review) but there is almost no research on how adolescent psychological 
characteristics beyond features of eating pathology might impact treatment outcome (Jewell et al., 2016a).

The present study sought to shed light on predictors of outcome and mechanisms of change in FT-AN by focussing on two constructs that are salient in eating disorders: attachment and mentalizing. Attachment can be understood as a broad, higher-order construct which has been operationalized in multiple ways, with a foundation in the conceptual and empirical work of Bowlby (1969) and Ainsworth et al. (1978); attachment style, the operationalization used in this study, refers to a constellation of knowledge, expectations, and insecurities that people hold about themselves and their close relationships (Fraley \& Roisman, 2019). Meta-analytic evidence suggests that individuals with eating disorders have higher rates of insecure attachment, and more difficulties mentalizing, than community controls (Caglar-Nazali et al., 2014; Jewell et al., 2016b). Moreover, attachment is an established predictor of both outcome and therapeutic alliance in adult psychotherapy (Bernecker et al., 2014; Levy et al., 2018).

In adult eating disorders, attachment security has been shown to predict positive therapeutic alliance (Folke et al., 2016) and differential response to treatment (Illing et al., 2010; Tasca \& Balfour, 2014), including rates of dropout, although attachment failed to predict outcome in recent studies by Kuipers et al. $(2017,2018)$ and Wallis et al. (2017b). Of note, the Wallis et al. study investigated attachment as a predictor of outcome in a secondary analysis of an RCT of Family-Based Treatment, using the Inventory of Parent and Peer Attachment (Armsden \& Greenberg, 1987). Whilst attachment was not predictive of outcome, the study was underpowered for the 
purposes of prediction, which is a limitation common to many of the studies investigating predictors of outcome in the field of FT-AN (Hamadi \& Holliday, 2020).

Mentalization refers to the capacity to understand others' actions as well as one's own behaviour in terms of intentional mental states such as feelings, desires, attitudes, and goals and is assumed to predict the capacity for emotion regulation (Luyten et al., 2020). Reflective function refers to this ability operationalized in the context of attachment relationships (Fonagy et al., 1991). Difficulties in reflective function can be conceptualized as falling into two broad domains: hypomentalizing refers to an inability to consider complex models of one's own mind and/or that of others, whereas hypermentalizing involves making unjustified assumptions about other people's mental states that go far beyond the observable data (Fonagy et al., 2016). Mentalization has been shown to predict alliance (Katznelson et al., 2020) and outcomes in individuals with eating disorders (Kuipers et al., 2017; Rothschild-Yakar et al., 2010b; Schulte-Rüther et al., 2012b).

\subsubsection{Proposed links between attachment, mentalization and treatment outcome}

A person's ability to mentalize effectively is not a static trait but is a dynamic capacity that is influenced by stress and arousal, particularly in the context of specific attachment relationships (Luyten et al., 2020). Under conditions of high arousal, patterns of brain activity 'switch' from flexibility to automaticity (Long et al., 2020), leading to pre-mentalizing modes of experiencing subjectivity, such as over-certain or concrete modes of thought (Fonagy \& Luyten, 2009). Attachment security, which raises the threshold for this switch-point to automatic and pre-mentalizing modes of thought, can thus be conceptualised as a protective factor for individuals experiencing 
affectively-charged occurrences within their attachment network (Luyten et al., 2020). We therefore hypothesised that attachment security and capacity for mentalization would be associated with positive treatment outcome in FT-AN, given that the process of attending treatment for, and recovering from, $\mathrm{AN}$ is associated with high levels of stress for adolescents and their families (White et al., 2020).

Attachment security was postulated as a relational strength that could help by increasing the likelihood of forming a positive therapeutic alliance in treatment. By contrast, insecure attachment may make family members vulnerable to experiencing pre-mentalizing modes of thought in the context of emotionally charged situations, including treatment sessions. Within this model, emotional regulation might also emerge as a predictor of outcome, given its theoretical and empirical associations with attachment and mentalizing (Sharp et al., 2011) and its relevance to the 'switch-point' theory.

\subsubsection{The potential role of emotional regulation}

Emotional regulation has been considered an important factor predicting the development of therapeutic alliance and eventual treatment outcome, given its theoretical and empirical associations with attachment and mentalizing (Fonagy, Gergely, Jurist, Gergely, et al., 2002; Sharp et al., 2011; Tasca et al., 2009), and its potential relevance to the switch-point theory described above. In addition, (Hallion et al., 2018) recently reported that baseline scores on the Difficulties in Emotion Regulation Scale (Gratz \& Roemer, 2004) were predictive of treatment outcome in 
Cognitive Behaviour Therapy (CBT) for adult emotional disorders. In this study, items loaded on to a general factor which was positively associated with better outcomes, meaning that higher scores (reflecting greater difficulties) predicted better response to treatment. However, greater impairment on the Goals subscale of the Difficulties in Emotion Regulation Scale, reflecting difficulties in engaging with goal-directed behaviour when upset, was negatively correlated with outcome. These findings speak to the importance of undertaking fine-grained analyses at subscale level, whilst also speaking to the need for further psychometric work to define constructs and refine measures.

\subsubsection{Hypotheses:}

The aim of the study is to address the following hypotheses:

1) Baseline insecure attachment, mentalizing difficulties and emotion regulation difficulties, in both adolescents and parents, will predict Morgan-Russell scale poor treatment outcome at nine months.

2) The association between attachment, mentalization and emotion regulation difficulties and treatment outcome will be mediated by the therapeutic alliance between family and therapist. Specifically, insecure attachment and difficulties in mentalizing and emotion regulation, as assessed in adolescents and parents at baseline, will be associated with lower self-reported therapeutic alliance with the therapist. Poorer alliance will be predictive of poor outcome. A visual depiction of these research questions can be seen in Figure 5.1. 
Figure 5.1 Conceptual diagram showing hypothesised model. Double headed arrows refer to correlations between variables

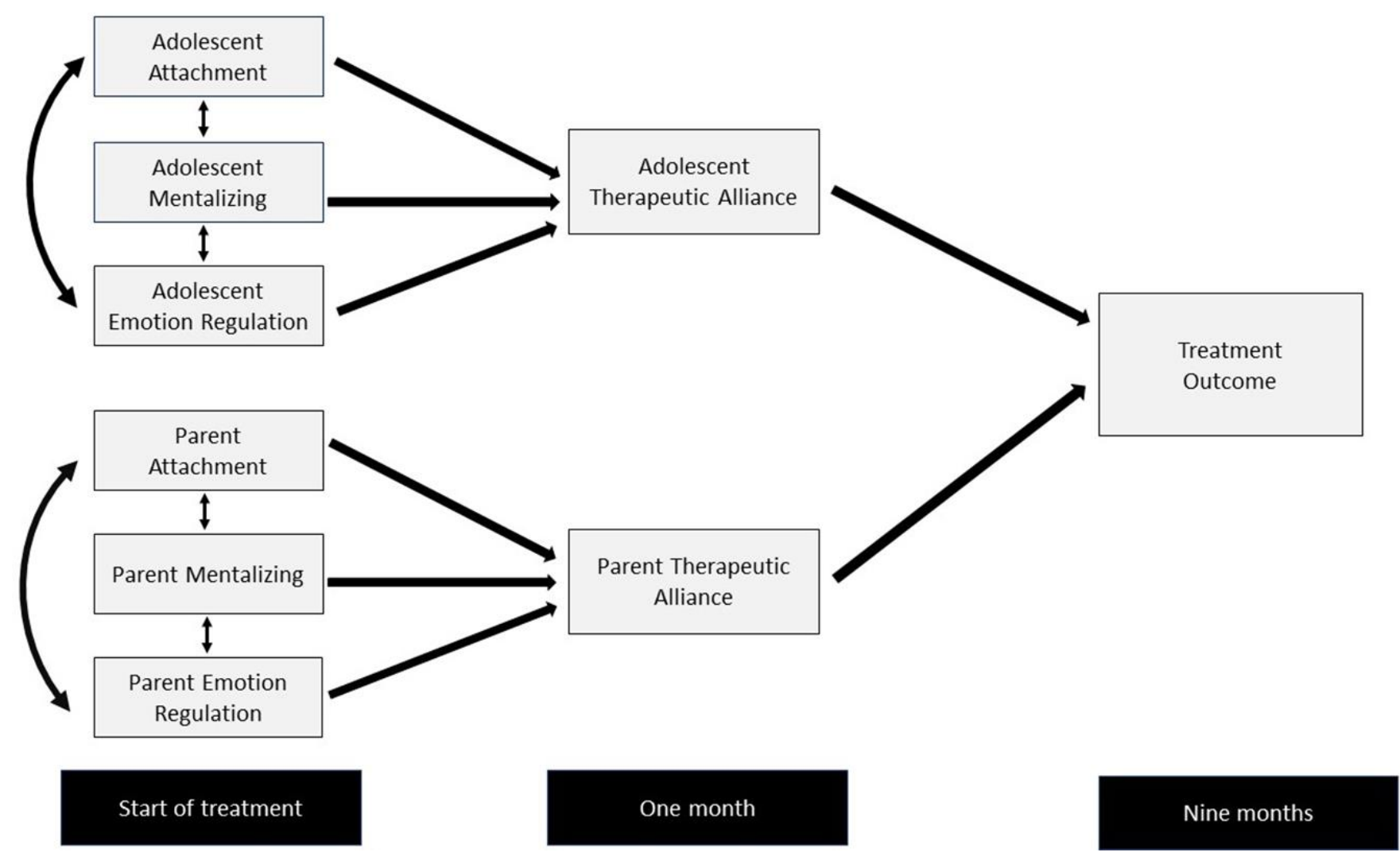




\section{$\underline{5.2 .4 \text { Secondary analysis }}$}

We will also conduct a secondary analysis to test the following hypothesis: Insecure attachment, mentalizing difficulties and emotion regulation difficulties, assessed at baseline in both adolescents and parents will predict lower gains in percentage median body mass index (\% mBMI) at 9 months.

\subsection{Methods}

Data were collected from three clinical sites in the United Kingdom, all of which are specialist eating disorder services based in two diverse, multi-ethnic cities. Adolescent patients and their families meeting criteria for the study were approached at assessment or start of treatment. All consecutive cases meeting eligibility within the data collection period were invited to participate. Patient inclusion criteria were as follows: aged between 10 and 17; living with their parent/s or carer/s for at least the previous three months; diagnosed at clinical assessment with anorexia nervosa (restricting or binge/purge subtype) using DSM-5 criteria (American Psychiatric Association, 2013); or meeting DSM-5 criteria for Other Specified Feeding or Eating Disorder (OSFED) providing their \% $\mathrm{mBMI}$ at assessment was at $85 \%$ or below, or the adolescent had lost $15 \%$ of their body weight in the three months prior to assessment; adequate level of English, i.e. sufficient to understand study information sheets and consent forms; receiving outpatient family therapy for anorexia nervosa (FT-AN) as their treatment. Parent/carer criteria were that they would be involved in attending FT-AN sessions and had an adequate level of English. At least one parent/carer needed to consent to take part in the study. Up to two parents/carers could be recruited per adolescent. 


\subsubsection{Data collection}

Self-report measures of attachment, mentalization and emotion regulation were completed at the start of treatment (T1) and one month into treatment (T2) by adolescent patients and at least one of their parents. At T2 self-report measures of alliance were also completed by parents and adolescents.

Young people and parents completed self-report measures online using the Bristol Online Survey, usually from their own homes (approximately 5\% of T1 questionnaires were completed on paper). Weight and height data were collected by clinicians as part of the routine clinical assessment. The conversions to percent median body mass index (\%mBMI) were done using a computer programme based on the (Child Growth Foundation, 1990) development charts.

\section{$\underline{\text { 5.3.2 Outcomes }}$}

Primary Outcome: Morgan-Russell scales as modified by (Russell et al., 1987), in which categorical outcome was recorded at 9 months at one of three levels, as follows: Good outcome - participants whose weight is above $85 \% \mathrm{mBMI}$, who are menstruating and have no bulimic symptoms; Intermediate outcome - participants meet the same weight criteria but are either not menstruating or have occasional bulimic symptoms (averaging less than once a week over the past month); Poor outcome - participants whose weight is below $85 \% \mathrm{mBMI}$ or have developed bulimic symptoms more than once a week, or are in inpatient care at 9 months. 
Weight data are reported as a percentage of median BMI (\%mBMI), adjusted for height, age and sex (Child Growth Foundation, 1990).

\subsubsection{Adolescent exposure variables (T1):}

- Attachment Style Questionnaire (ASQ - Feeney et al., 1994). This 40-item attachment measure was developed for use with both adults and adolescents and is comprised of five subscales: Confidence, Preoccupation with Relationships, Need for Approval, Relationships as Secondary and Discomfort with Closeness. Internal reliability and test-retest reliability are both good.

- Difficulties in Emotion Regulation Strategies Scale (DERS - Gratz \& Roemer, 2004). This 36-item measure assesses emotion regulation difficulties and is comprised of six subscales: Non-acceptance of Emotional Responses, Difficulties Engaging in Goal-directed Behaviors, Impulse Control Difficulties, Lack of Emotional Awareness, Limited Access to Effective Emotion Regulation Strategies and Lack of Emotional Clarity. The measure has demonstrated high internal consistency and good construct and predictive validity in clinical and non-clinical populations (Gratz \& Roemer, 2004).

- Hypermentalizing Questionnaire - Adolescent version (parent scale) (Sharp et al., 2018). This scale comprises 26 items which assess adolescent hypermentalizing in their interaction with parents (e.g. "I worry a lot about what my parents are thinking and feeling"). The measure yields a total Hypermentalizing score.

- Reflective Function Questionnaire - Youth (RFQY - Ha et al., 2013). This 46-item measure yields a Total score, with higher scores representing greater reflective function (sample item: "I always know what I feel"). The RFQY has 
adequate internal reliability. Convergent, criterion, discriminant and construct validity have been demonstrated (Ha et al., 2013).

\section{$\underline{\text { 5.3.4 Parent exposure variables (T1) }}$}

- Reflective Function Questionnaire (RFQ8 - Fonagy et al., 2016). This 8-item measure yields two subscales: Certainty about Mental States (henceforth referred to as Certainty), and Uncertainty about Mental States (henceforth: Uncertainty). Scoring is applied such that extreme scores at either end of the Likert scale are indicative of inadequate mentalizing. For instance, strong agreement with the item "I always know what I feel" yields a higher score on the Certainty scale, whereas strong agreement with the item "I don't always know why I do what I do" yield a higher score on the Uncertainty scale. The measure has demonstrated satisfactory levels of internal validity, convergent validity and test-retest reliability (Fonagy et al., 2016).

- Attachment Style Questionnaire (Feeney et al., 1994).

- Difficulties in Emotion Regulation Strategies Scale (Gratz \& Roemer, 2004).

- Hypermentalizing Questionnaire - Parent version (adolescent scale) (Sharp et al., 2018). Parents completed the adolescent scale of the Hypermentalizing Questionnaire, which assesses parental hypermentalizing in their interaction with their adolescent (e.g. "My adolescent often says I over interpret his/her behavior or words"). 


\section{$\underline{\text { 5.3.5 Adolescent and parent mediator variables (T2) }}$}

- System for Observing Family Therapy Alliance - Self-report (SOFTA Friedlander et al., 2006). This 16-item measure assesses perceptions of family therapy alliances on four subscales: Engagement in the Therapeutic Process, Emotional Connection to the Therapist; Safety within the Therapeutic System and Shared Sense of Purpose within the Family. Internal reliability for the total scale is good, and adequate-to-good for the four subscales. Convergent validity with an observer-rated version of the SOFTA has been demonstrated (Friedlander et al., 2006).

\section{$\underline{\text { 5.3.6 Baseline covariates }}$}

- $\quad$ Eating Disorder Examination Questionnaire (EDE-Q - Fairburn \& Beglin, 1994). This widely used 28-item measure of eating disorder pathology has good internal consistency, test-retest reliability and discriminant validity (see (Berg et al., 2012) for a review). The EDE-Q Global Score completed at initial clinical assessment was extracted from patient records.

- Baseline percentage median Body Mass Index (\% mBMI) adjusted for age and gender (Child Growth Foundation, 1990).

- Duration of illness in months. This refers to the number of months that the young person had been unwell for prior to their assessment in clinic.

EDE-Q was completed by adolescents at assessment (prior to entry to the study). Data on baseline percentage BMI and duration of illness was extracted from clinical notes from the time of clinical assessment. 


\subsubsection{Baseline measures of comorbidity}

- $\quad$ Revised Child Anxiety and Depression Scale (RCADS) - Youth self-report version (Chorpita et al., 2000). This 47-item questionnaire is a wellestablished measure with good to excellent internal consistency, good testretest reliability, and strong convergent and divergent validity (Chorpita et al., 2005). The Total Internalizing Scale (including both depression and anxiety subscales) completed at initial clinical assessment was extracted from patient records.

- Self-harm at assessment - this was based on adolescent report at clinical assessment and was extracted from patient records, coded as 1 (for present) or 0 (not present).

\section{$\underline{\text { 5.3.8 Study registration }}$}

The study was pre-registered with Europe Pubmed Central, with a brief overview of the study available at http://europepmc.org/grantfinder/grantdetails?query=pi\%3A\%22Jewell\%20T\%22\% 20gid\%3A\%22CDRF-2014-05-024\%22\%20ga\%3A\%22DH\%2FNIHR\%22.

\section{$\underline{\text { 5.3.9 Ethics }}$}

The study was approved by the Camden and King's Cross ethics committee of the Health Research Authority. 


\section{$\underline{\text { 5.3.10 Sample size }}$}

The study aimed to recruit 200 participants. The power calculation assumptions were that a sample size of 200 with follow-up of $80 \%$ at 9 months and $30 \%$ experiencing poor treatment outcome, would have $80 \%$ power to detect an odds ratio (OR) of $>1.58$ in relation to a one standard deviation difference in the predictor variable in the logistic regression analysis with an alpha-level of 0.05 .

\section{$\underline{\text { 5.3.11 Internal reliability }}$}

We report McDonald's Omega for all self-report measures in Table 2. McDonald's Omega was chosen since it is considered a less biased metric of internal consistency than Cronbach's alpha. For the EDEQ and RCADS data we did not have item-level data available for our participants, as these scores were extracted from patient notes. Therefore, McDonald's Omega for these two measures were calculated from routine outcome databases from Site 1.

\section{$\underline{\text { 5.3.12 Public and Patient Involvement (PPI) }}$}

Parents and adolescents with lived experience of AN were involved in the design and interpretation of this study. The choice of measures and methods for approaching and recruiting participants were informed by PPI feedback. To guide interpretation, a public meeting was held in August 2019 to share findings with those with lived experience, which was followed up by dissemination over email of lay summaries in late 2019. The findings were discussed over email and telephone with several individuals with lived experience. Suggestions from these activities are included in the discussion section. 


\section{$\underline{\text { 5.3.13 Analysis plan }}$}

The analyses were conducted in two stages:

For the purposes of the main analysis the primary outcome was a binary variable combining Good and Intermediate outcome to compare with Poor outcome on the Morgan Russell scales.

1. Binary logistic regression was used to test the association between predictors (at baseline) and therapy outcome at 9 months after treatment began. Robust standard errors were used in all analyses due to non-independence of participants due to clustering by site. Alpha level was set at 0.05 . Predictor variables were tested at the subscale level and using total scores where available.

All analyses included the following covariates recorded at the time of clinical assessment: age (in years); duration of illness (in months) at the time of assessment; severity of eating pathology using the EDEQ Global score (Fairburn \& Beglin, 1994b); baseline percentage median Body Mass Index adjusted for age and gender; and treatment site.

2. Mediation analyses following the (Baron \& Kenny, 1986) approach:

i) Binary logistic regression on outcome of baseline predictor variables plus covariates (effect of exposures on outcome).

ii) Linear regression on alliance (the hypothesised mediator) plus covariates (effect of exposures on mediator).

iii) Binary logistic regression on outcome of baseline predictor variables, plus mediator, plus covariates (effect of exposure and mediator on outcome). 
Mediation could only be considered to be present if there were significant relationships between baseline predictors and both outcome (i) and alliance (ii). In step (iii), full mediation would be present if baseline predictors were no longer significant after adding alliance to the model, whilst partial mediation would be present if baseline predictors remained significant but with a reduced effect size.

\section{$\underline{\text { 5.3.14 Missing data }}$}

Missing data for individual items on the self-report measures of predictor variables was low since most questionnaires were completed online, with participants unable to submit their responses if items were missing. Approximately 5\% of participants completed paper questionnaires at Time 1. Missing data were more frequent for Time 2 data, which included self-report measures of alliance, the hypothesised mediator. There were also higher rates of missing data for some baseline covariate measures, such as the RCADS and EDEQ measures, which were drawn from data collected routinely in the clinics (see Table 1 for details). To minimise bias arising from complete case analysis, we used multiple imputation to impute missing values.

\section{$\underline{\text { 5.3.15 Multiple imputation }}$}

We analysed patterns of missingness for baseline covariates, Time 2 alliance scores and Time 5 percentage median body mass index by creating new binary variables to indicate completeness/missingness for each variable of interest. Binary logistic regression was used to identify significant predictors of missingness. We used multiple imputation with chained equations (Royston \& White, 2011) and entered the following significant predictors of missingness: the EDEQ Global score; the RCADS 
Internalising total score; adolescent Attachment Style Questionnaire Confidence and Preoccupation scales; the adolescent Difficulties in Emotion Regulation Scale Total score; the Reflective Function Scale - Youth total score, and the parental Attachment Style Questionnaire Preoccupation Scale. Auxiliary variables were age, baseline percentage median body mass index, site, sex, outcome, and use of higher level of care.

We imputed missing baseline covariate, Time 2 alliance scores and 9-month \%mBMI median body mass index, using the MI impute chained command in Stata and 50 imputed datasets. All reported analyses of the primary and secondary outcome have been run using multiple imputation. All analyses were conducted in Stata v.15 (StataCorp, College Station, TX). 


\subsection{Results}

\section{$\underline{\text { 5.4.1 Demographics }}$}

We recruited 192 adolescents and their parent/s to the study. Of this total, we excluded 19 participants (9.9\% of the sample) for the following reasons: twelve participants did not complete any measures for the study; two participants withdrew their consent; four were recruited to the study in error without meeting inclusion criteria; one participant had baseline data but no outcome data. The recruitment flowchart for the study is presented in Figure 5.2. Recruitment took place from September 2015 to July 2018 in Site 1; February 2016 to July 2018 in Site 2; and January 2017 to July 2018 in Site 3.

The final sample for whom baseline and outcome data were available comprised 173 adolescents, of whom $153(88.4 \%)$ were female, with a mean age of 14.7 years. The parent sample comprised $n=163$, of whom 14 parents were fathers and 149 parents were mothers. No parent data was available for ten adolescent participants. For 17 participants data were available from both parents, as both had been recruited to the study. Since the majority of the parent sample were mothers, and to prevent artificially inflating the sample size beyond the 173 adolescents in the study, we excluded data from fathers in the 17 cases where mother data were available. Data from fathers were used for 14 adolescents who did not have data from mothers.118 participants came from Site 1, 43 from Site 2, and 12 from Site 3. Demographic data for the sample are presented in Table 5.1. Thirty-four adolescents (19.7\%) attended some form of higher level of care, including $18(10.4 \%)$ who attended day-patient care, $11(6.4 \%)$ who were admitted to inpatient psychiatric care, and $11(6.2 \%)$ who were admitted to a paediatric ward. 
Figure 5.2 Recruitment flowchart

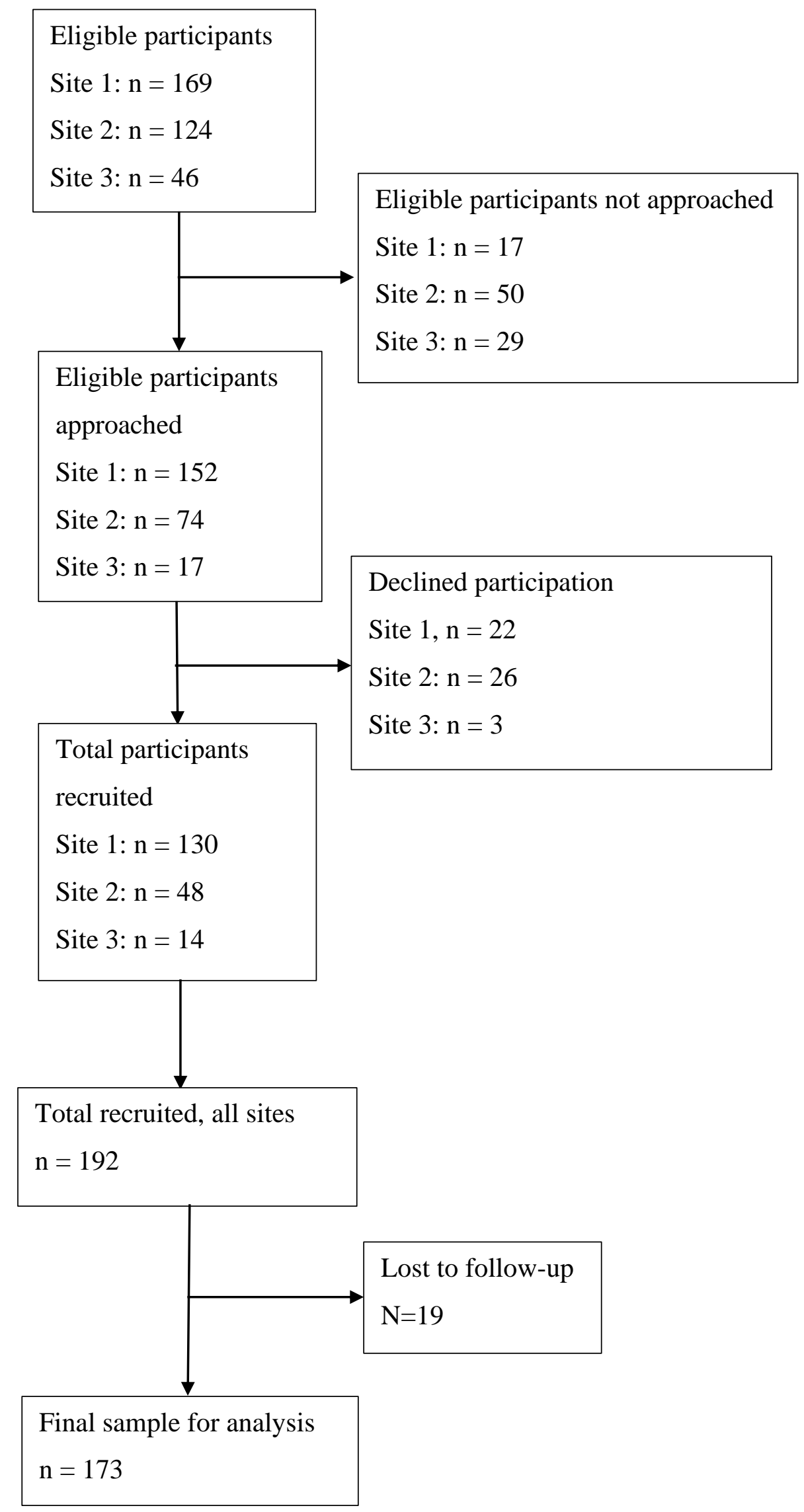


Table 5.1 Demographic characteristics of the sample

\begin{tabular}{|c|c|c|c|c|c|}
\hline & \\
\hline & $\mathrm{N}$ & Minimum & Maximum & Mean & SD \\
\hline Age & 173 & 11 & 17 & 14.69 & 1.54 \\
\hline$\%$ Median Body Mass Index & 173 & 62.27 & 113.79 & 83.89 & 8.97 \\
\hline \multirow[t]{2}{*}{ Duration of illness (Months) } & 170 & 1 & 65 & 10.48 & 10.59 \\
\hline & $\mathrm{N}$ & $\%$ & & & \\
\hline \multicolumn{6}{|l|}{ Sex: } \\
\hline - Female & 153 & 88.4 & & & \\
\hline - Male & 20 & 11.6 & & & \\
\hline \multicolumn{6}{|l|}{ Lives with: } \\
\hline - Natural/ adoptive parents & 129 & 74.6 & & & \\
\hline - Mother alone & 24 & 13.9 & & & \\
\hline - Other & 20 & 11.6 & & & \\
\hline
\end{tabular}

Ethnicity:

- White British

$138 \quad 79.8$

$\begin{array}{lll}\text { - Other White } & 13 & 7.5\end{array}$

$\begin{array}{lll}\text { - Dual Heritage } & 10 & 5.8\end{array}$

- British Asian/Other South Asian $\quad 9 \quad 5.2$

$\begin{array}{lll}\text { - Other } & 3 & 1.7\end{array}$

Diagnosis: - Anorexia Nervosa (restricting sub-
type)

- Anorexia Nervosa (binge/purge sub- $\quad 9 \quad 5.2$ type)

$\begin{array}{lll}\text { - OSFED } & 24 & 13.9\end{array}$

$\begin{array}{lll}\text { Self-harm reported at assessment } & 35 & 20.2\end{array}$ 
Table 5.2 Range, Means, Standard Deviations and Internal Reliability of Self-Report Measures

\begin{tabular}{|c|c|c|c|c|c|c|}
\hline Adolescents & $\mathbf{N}$ & Min. & Max. & Mean & SD & Omega \\
\hline RCADS Total Internalising (T1) & 127 & 3 & 136 & 55.21 & 27.33 & 0.97 \\
\hline EDE-Q Global (T1) & 159 & .00 & 5.70 & 3.35 & 1.56 & 0.95 \\
\hline ASQ Confidence (T1) & 170 & 9 & 48 & 28.88 & 7.25 & 0.86 \\
\hline ASQ Discomfort (T1) & 170 & 10 & 58 & 39.83 & 8.86 & 0.88 \\
\hline ASQ Secondary (T1) & 170 & 7 & 38 & 19.59 & 5.94 & 0.80 \\
\hline ASQ Need for Approval (T1) & 170 & 11 & 42 & 30.44 & 5.81 & 0.77 \\
\hline ASQ Preoccupation (T1) & 170 & 8 & 46 & 29.82 & 6.70 & 0.76 \\
\hline DERS Nonacceptance (T1) & 170 & 6 & 30 & 18.60 & 7.56 & 0.94 \\
\hline DERS Goals (T1) & 170 & 5 & 25 & 17.98 & 5.20 & 0.89 \\
\hline DERS Impulse (T1) & 170 & 6 & 30 & 17.62 & 6.82 & 0.92 \\
\hline DERS Awareness (T1) & 170 & 6 & 30 & 17.62 & 5.10 & 0.81 \\
\hline DERS Strategies (T1) & 170 & 8 & 40 & 25.78 & 8.62 & 0.93 \\
\hline DERS Clarity (T1) & 170 & 5 & 25 & 14.58 & 4.98 & 0.89 \\
\hline HMZ Total (T1) & 169 & 2 & 94 & 53.56 & 18.41 & 0.92 \\
\hline RFQY (T1) & 170 & 6.26 & 10.44 & 8.78 & .79 & 0.76 \\
\hline SOFTA Engagement (T2) & 125 & 4 & 20 & 12.36 & 3.62 & 0.75 \\
\hline SOFTA Connection (T2) & 125 & 4 & 20 & 14.34 & 4.00 & 0.84 \\
\hline SOFTA Safety (T2) & 125 & 4 & 20 & 11.52 & 3.70 & 0.70 \\
\hline SOFTA Purpose (T2) & 125 & 6 & 20 & 15.04 & 3.32 & 0.72 \\
\hline Parents & $\mathbf{N}$ & Min. & Max. & Mean & SD & Omega \\
\hline ASQ Confidence (T1) & 163 & 12 & 47 & 36.06 & 5.58 & 0.82 \\
\hline ASQ Discomfort (T1) & 163 & 11 & 53 & 31.93 & 8.41 & 0.88 \\
\hline ASQ Secondary (T1) & 163 & 7 & 28 & 14.57 & 4.62 & 0.72 \\
\hline ASQ Need (T1) & 162 & 7 & 36 & 20.73 & 5.60 & 0.78 \\
\hline ASQ Preoccupation (T1) & 163 & 11 & 47 & 24.67 & 6.67 & 0.81 \\
\hline DERS Nonacceptance (T1) & 163 & 6 & 30 & 11.42 & 5.14 & 0.88 \\
\hline DERS Goals (T1) & 163 & 5 & 25 & 12.56 & 4.50 & 0.86 \\
\hline DERS Impulse (T1) & 163 & 6 & 28 & 10.15 & 4.38 & 0.89 \\
\hline DERS Awareness (T1) & 163 & 6 & 26 & 14.49 & 4.37 & 0.79 \\
\hline DERS Strategies (T1) & 163 & 8 & 39 & 13.83 & 5.43 & 0.88 \\
\hline DERS Clarity (T1) & 163 & 5 & 20 & 8.79 & 2.92 & 0.80 \\
\hline RFQ8 Certainty (T1) & 163 & .00 & 3.00 & 1.22 & .74 & 0.79 \\
\hline RFQ8 Uncertainty (T1) & 163 & .00 & 2.17 & .41 & .45 & 0.76 \\
\hline Hypermentalizing Total (T1) & 162 & 8 & 81 & 39.78 & 17.55 & 0.93 \\
\hline SOFTA Engagement (T2) & 132 & 8 & 20 & 15.86 & 2.65 & 0.67 \\
\hline SOFTA Connection (T2) & 132 & 7 & 20 & 15.77 & 2.83 & 0.77 \\
\hline SOFTA Safety (T2) & 132 & 8 & 20 & 15.97 & 2.76 & 0.66 \\
\hline SOFTA Purpose (T2) & 132 & 8 & 20 & 16.83 & 2.85 & 0.75 \\
\hline
\end{tabular}


Note. Abbreviations in table: RCADS, Revised Child Anxiety and Depression Scale (RCADS); EDE-Q, Eating Disorder Examination Questionnaire; ASQ, Attachment Style Questionnaire; ASQ Discomfort, ASQ Discomfort with Closeness; ASQ Preoccupation, ASQ Preoccupation with Relationships; ASQ Secondary, ASQ Relationships as Secondary; DERS, Difficulties in Emotion Regulation Strategies Scale; DERS Nonacceptance, DERS Nonacceptance of Emotional Responses; DERS Goals, DERS Difficulties Engaging in Goal-Directed Behaviors; DERS Impulse, DERS Impulse Control Difficulties; DERS Awareness, DERS Lack of Emotional Awareness; DERS Strategies, DERS Limited Access to Effective Emotion Regulation Strategies; DERS Clarity, DERS Lack of Emotional Clarity; RFQY, Reflective Function Questionnaire - Youth Total Score; SOFTA, System for Observing Family Therapy Alliance; SOFTA Engagement, SOFTA Engagement in the Therapeutic Process; SOFTA Connection, SOFTA Emotional Connection to the Therapist; SOFTA Safety, SOFTA Safety within the Therapeutic System; SOFTA Purpose, SOFTA Shared Sense of Purpose within the Family; RFQ8, Reflective Function Questionnaire (8 item version); RFQ8 Certainty, RFQ8 Certainty About Mental States; RFQ8 Uncertainty, RFQ8 Uncertainty About Mental States. Omega refers to McDonald's Omega.

\subsubsection{Descriptive statistics}

The range, means and standard deviations for self-report measures are presented in

Table 5.2. Tables showing further breakdowns of the descriptive data by parental gender, adolescent gender and by site are available in Appendices G, H and I respectively.

\section{$\underline{\text { 5.4.3 Correlations between baseline variables }}$}

Pearson's correlations between key baseline and exposure variables are reported in Table 5.3. A larger table reporting correlations between baseline, exposure and mediator variables can be found as an Excel table in a supplementary table (S1) available at https://osf.io/yevg2/files/ 
Table 5.3 Correlations between key baseline and exposure variables

\begin{tabular}{|c|c|c|c|c|c|c|c|c|c|c|c|c|c|}
\hline & & & 1 & 2 & 3 & 4 & 5 & 6 & 7 & 8 & 9 & 10 & 11 \\
\hline & EDEQ Global & Pearson's r & - & & & & & & & & & & \\
\hline & & p-value & - & & & & & & & & & & \\
\hline \multirow[t]{2}{*}{2.} & $\% \mathrm{mBMI}$ & Pearson's r & 0.293 & - & & & & & & & & & \\
\hline & & $\mathrm{p}$-value & $<.001$ & - & & & & & & & & & \\
\hline \multirow[t]{2}{*}{3.} & Adolescent ASQ Confidence & Pearson's r & -0.214 & -0.065 & - & & & & & & & & \\
\hline & & p-value & 0.008 & 0.399 & - & & & & & & & & \\
\hline \multirow[t]{2}{*}{4.} & Adolescent DERS Total & Pearson's r & 0.392 & 0.112 & -0.615 & - & & & & & & & \\
\hline & & p-value & $<.001$ & 0.146 & $<.001$ & - & & & & & & & \\
\hline \multirow[t]{2}{*}{5.} & Adolescent RFOY & Pearson's $\mathrm{r}$ & -0.097 & -0.141 & 0.230 & -0.228 & - & & & & & & \\
\hline & & $\mathrm{p}$-value & 0.230 & 0.067 & 0.003 & 0.003 & - & & & & & & \\
\hline \multirow[t]{2}{*}{6.} & Adolescent Hypermentalizing & Pearson's r & 0.296 & 0.057 & -0.550 & 0.694 & -0.152 & - & & & & & \\
\hline & & p-value & $<.001$ & 0.458 & $<.001$ & $<.001$ & 0.049 & - & & & & & \\
\hline & Parent ASQ Confidence & Pearson's r & 0.109 & 0.036 & 0.133 & -0.163 & -0.029 & -0.147 & - & & & & \\
\hline & & p-value & 0.185 & 0.646 & 0.094 & 0.039 & 0.720 & 0.064 & - & & & & \\
\hline & Parent DERS Total & Pearson's r & 0.057 & 0.203 & -0.194 & 0.224 & -0.095 & 0.212 & -0.495 & - & & & \\
\hline & & p-value & 0.485 & 0.009 & 0.014 & 0.004 & 0.231 & 0.007 & $<.001$ & - & & & \\
\hline \multirow[t]{2}{*}{9.} & Parent RFQ8 Certainty & Pearson's r & -0.146 & -0.111 & 0.157 & -0.244 & 0.026 & -0.253 & 0.296 & -0.487 & - & & \\
\hline & & p-value & 0.075 & 0.160 & 0.048 & 0.002 & 0.745 & 0.001 & $<.001$ & $<.001$ & 一 & & \\
\hline \multirow{2}{*}{\multicolumn{2}{|c|}{ 10. Parent RFQ8 Uncertainty }} & Pearson's r & 0.140 & 0.238 & -0.082 & 0.118 & -0.053 & 0.155 & -0.215 & 0.517 & -0.626 & - & \\
\hline & & p-value & 0.087 & 0.002 & 0.305 & 0.137 & 0.506 & 0.051 & 0.006 & $<.001$ & $<.001$ & - & \\
\hline \multirow{2}{*}{\multicolumn{2}{|c|}{ 11. Parent Hypermentalizing }} & Pearson's r & 0.205 & 0.220 & -0.207 & 0.324 & -0.051 & 0.344 & -0.195 & 0.572 & -0.526 & 0.448 & - \\
\hline & & $\mathrm{p}$-value & 0.012 & 0.005 & 0.009 & $<.001$ & 0.527 & $<.001$ & 0.013 & $<.001$ & $<.001$ & $<.001$ & 一 \\
\hline
\end{tabular}


Note. Abbreviations in table: EDE-Q Global, Eating Disorder Examination Questionnaire Global score; ASQ, Attachment Style Questionnaire; DERS Total, Difficulties in Emotion Regulation Strategies Scale total score; RFQY, Reflective Function Questionnaire - Youth total Score; Hypermentalizing, Hypermentalizing Questionnaire total score; RFQ8, Reflective Function Questionnaire (8 item version); RFQ8 Certainty, RFQ8 Certainty About Mental States; RFQ8 Uncertainty, RFQ8 Uncertainty About Mental States. 


\subsubsection{Prediction of outcome from baseline covariates}

A higher baseline \%mBMI $(\mathrm{n}=173, \mathrm{OR}=1.11$, CI: $1.04-1.18)$ and higher selfreported eating pathology (EDEQ Global score) $(n=159$, OR $=1.36$, CI: $1.01-1.83)$ were associated with higher odds of positive outcome. Older age $(n=173$, OR: 0.63 , CI: $0.48-0.82)$ and longer duration of illness $(n=170$, OR: 0.97, CI: $0.94-1.00)$ were associated with higher odds of poor outcome.

\subsubsection{Associations between exposure, mediator and outcome variables}

The associations between all exposure and mediator variables with treatment outcome are available in Appendices $\mathrm{J}$ and $\mathrm{K}$ for parents and adolescents respectively. In the following sections we highlight the significant findings for each pathway.

\subsubsection{Prediction of outcome from baseline exposure variables}

Of the parent measures, higher scores on the RFQ8 Certainty subscale were predictive of poor outcome $(n=163, \mathrm{OR}=0.42, \mathrm{CI}: 0.20-0.87)$, whilst higher scores on the DERS Impulsive subscale $(\mathrm{n}=163, \mathrm{OR}=1.23, \mathrm{CI}$ : 1.07 - 1.43) and Hypermentalizing Questionnaire $(\mathrm{n}=162, \mathrm{OR}=1.03, \mathrm{CI}: 1.00-1.06)$ predicted positive outcome. Of the adolescent measures, higher scores on the DERS Lack of Clarity scale, in which higher scores represent being unclear about one's feelings, predicted positive outcome $(\mathrm{n}=173$, OR $=1.10$, CI: $1.00-1.21)$.

\subsubsection{Prediction of outcome by alliance (hypothesised mediator)}

Of the SOFTA subscales, positive outcome was predicted by parental Emotional Connection score $(\mathrm{n}=173, \mathrm{OR}=1.32, \mathrm{CI}$ : $1.09-1.58)$, parental Sense of Safety score $(\mathrm{n}=173, \mathrm{OR}=1.24, \mathrm{CI}: 1.02-1.50)$ and the adolescent Shared Sense of Purpose score $(\mathrm{n}=173, \mathrm{OR}=1.18, \mathrm{CI}: 1.01-1.38)$. 


\subsubsection{Prediction of alliance by baseline exposure variables}

Of the SOFTA subscales that were predictive of outcome, parental SOFTA Sense of Safety scores were predicted by parental ASQ Confidence $(n=163, \beta=.17$, CI: .07 .26), parental ASQ Preoccupation $(\mathrm{n}=173, \beta=-.11$, CI: $-.19--.03)$, parental ASQ Need for Approval $(\mathrm{n}=162, \beta=-.10, \mathrm{CI}:-.19--.01)$ and parental DERS Goals subscale $(\mathrm{n}=163, \beta=-.15, \mathrm{CI}:-.26--.03)$. No exposure variables predicted parental SOFTA Emotional Connection scores.

For adolescents, the SOFTA Shared Sense of Purpose score was predicted by the adolescent DERS Awareness subscale $(\mathrm{n}=170, \beta=-.15$, CI: $-.28--.02)$ and RFQY score $(\mathrm{n}=173, \beta=1.02, \mathrm{CI}: .19-1.85)$.

\section{$\underline{\text { 5.4.10 Mediation analysis }}$}

Alliance scores did not mediate the relationship between the significant baseline exposure variables and treatment outcome. Using Generalized Structural Equation Modelling, we tested mediation models for the significant baseline exposure variables and significant alliance variables. For purposes of illustration, Figure 5.3 presents a mediation model including parental exposure and mediator variables, using the variables with the largest effect size for each pathway. As can be seen, whilst both the exposure (RFQ8 Certainty: $\mathrm{n}=163 \mathrm{OR}=.42$, CI: $.20-.87, \mathrm{p}=.02$ ) and mediator variables (SOFTA Emotional Connection: $\mathrm{n}=173, \mathrm{OR}=1.32, \mathrm{CI}: 1.09-1.58, \mathrm{p}<$ .01) are predictive of outcome, there is no significant association between the exposure and mediator variables (RFQ8 Certainty to SOFTA Emotional Connection: $\beta=-.13$, CI: $-.84-.58, \mathrm{p}=.71)$. 
Figure 5.3 Mediation model showing associations between parental exposure, parental mediator and outcome. Statistical values for significant pathways are in bold.

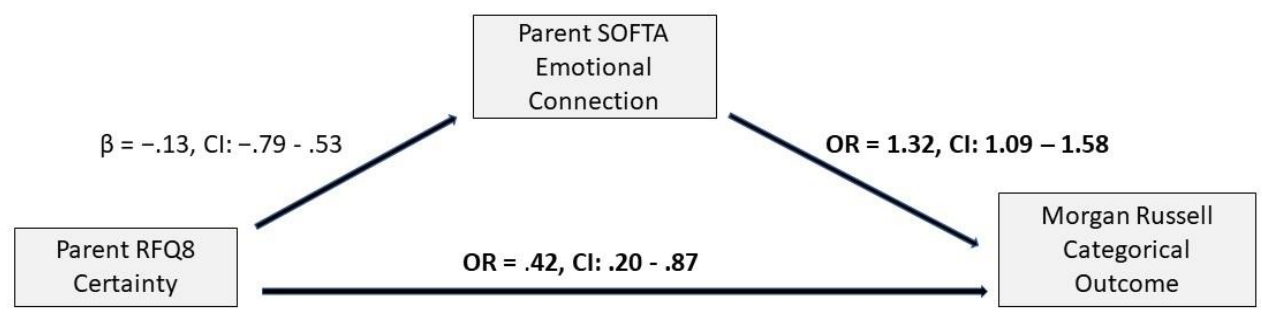

Abbreviations: RFQ8, Reflective Function Questionnaire (8 item version); SOFTA, System for Observing Family Therapy Alliance; OR, Odds Ratio; CI, Confidence Interval.

$\underline{\text { 5.4.11 Secondary analysis - prediction of percentage median Body Mass Index at } 9}$

$\underline{\text { months }}$

No parent or adolescent exposure or mediator variables predicted \%mBMI at 9 months.

\subsubsection{Discrepancies with complete case analysis}

Analyses were re-run with complete cases. The following results were discrepant with the imputed results reported in this paper:

Prediction of outcome from baseline exposure variables - Parental RFQ8 Certainty $(n=145)$ and parental Hypermentalizing $(n=144)$ were no longer significant.

Prediction of outcome by alliance - Adolescent SOFTA Purpose $(\mathrm{n}=111)$ was no longer significant. Parental SOFTA Engagement scores predicted outcome $(n=116$, $\mathrm{OR}=1.29$, CI: $1.01-1.66)$.

Prediction of alliance from baseline exposure variables - Parental SOFTA Total scores were predicted by parental DERS Lack of Emotional Clarity $(n=118, \beta=-.58$, CI: $-1.15--.01)$ and parental Hypermentalizing $(\mathrm{n}=118, \beta=-.10, \mathrm{CI}:-.20--.00)$. 
Parental SOFTA Emotional Connection scores were predicted by parental ASQ Confidence scores $(\mathrm{n}=118, \beta=.11, \mathrm{CI}: .01-.21)$. Negative associations of small magnitude were found between the following parental exposure variables and parental SOFTA Sense of Safety scores: ASQ Discomfort ( $\mathrm{n}=118, \beta=-.09, \mathrm{CI}:-.15--.02)$, Hypermentalizing Questionnaire $(\mathrm{n}=118, \beta=-.04, \mathrm{CI}:-.06--.01)$, DERS Lack of Emotional Clarity $(\mathrm{n}=118, \beta=-.25, \mathrm{CI}:-.40--.09)$, and DERS Strategies $(\mathrm{n}=118, \beta$ $=-.12$, CI: $-.22--.02$ ). For adolescents, SOFTA Shared Sense of Purpose Scores were predicted by adolescent ASQ Confidence $(n=114, \beta=.08$, CI: $.00-.16)$ and ASQ Preoccupation $(n=114, \beta=-.11, C I:-.22--.01)$. 


\subsection{Discussion}

Our study investigated the hypothesis that attachment and mentalizing would predict outcome in FT-AN, with therapeutic alliance serving as a mediator. Whilst the alliance was predictive of outcome in our data, and attachment and mentalizing were predictive of alliance, our theoretical model was not supported overall. Instead, our results suggest that excessive certainty about mental states, a variable uncorrelated with alliance, was the strongest predictor of poor outcome, with each unit increase on the RFQ8 Certainty scale reducing the odds of a good outcome by more than half. For adolescents, higher scores on the DERS Lack of Clarity subscale also emerged as a predictor of outcome. Whilst this finding was in the opposite direction to our hypotheses, it is consistent with our findings for parents, since extreme clarity about one's feelings is conceptually similar to certainty about mental states, and indeed we found that parental RFQ8 Certainty and adolescent DERS Lack of Emotional Clarity scores are negatively correlated in our data $(r=-.28, \mathrm{p}<.01)$.

Hallion et al. (2018) recently found higher scores on the DERS to be predictive of positive treatment outcomes in adults with emotional disorders. One possibility is that measures such as the DERS and RFQ8 might tap an adaptive process such as psychological insightfulness, which has been conceptualised as a construct under the overarching umbrella of mentalizing (Luyten et al., 2020). Such a view is supported by the positive association between self-reported eating pathology and good treatment outcome in our data. Similarly, our unexpected findings that higher self-reported parental hypermentalizing and impulse control difficulties predict positive outcome might be similarly explained by higher insightfulness. Alternatively, overly certain mentalizing might be associated with symptoms of autism, which are known to be 
elevated in individuals with anorexia nervosa, including adolescents (Westwood et al., 2018), and which are predictive of longer duration of illness in AN (Saure et al., 2020). In terms of mediating mechanisms, we speculate that excessive certainty might be correlated with both rigidity and difficulties in considering alternative perspectives. This might hamper recovery through reliance on a limited repertoire of problemsolving approaches, including the repeated application of unsuccessful strategies. However, given increasing evidence for the genetic and biological mechanisms underlying anorexia nervosa (Watson et al., 2019), excessive certainty may not be a learnt response, it could be a marker for biological risk domains, and represent a psychological mechanism by which genetic vulnerability confers higher risk of poorer outcome.

A priority for future research should be to better characterize adolescents and families who are at risk of poor outcome using diverse measures of constructs related to mentalizing and emotion-processing, assessed across different methods, including self-report, observational and experimental paradigms (Coniglio et al., 2019). Research on measurement should also be prioritised in order to improve the construct validity, cross-cultural validity and psychometric properties of measures across a range of domains, including alliance, emotion dysregulation, attachment and mentalization. The mechanisms by which excessive certainty might contribute to poorer outcome require elucidation and should include research into symptoms of autism and genetic profile. Relatedly, research is needed to investigate adaptations to treatment for individuals and families at risk of poorer outcome, but also for patients and families with differing needs. One example of this is the recent work undertaken to adapt treatment for adult patients with eating disorders who are on the autism spectrum, as highlighted in recent qualitative work (Adamson et al., 2020). Beyond 
neurodiversity, treatment needs may also differ for patients and families from other minority groups, including minority ethnic groups who at present face substantial systemic barriers to accessing treatment, and may benefit from culturally adapted treatment (Rodgers et al., 2018). Efforts to integrate new concepts such as mentalization into psychological treatments need to take place alongside research into the salience, meaning and measurement invariance of such concepts across a diverse range of individuals, as opposed to the Western, Educated, Industrialized, Rich, and Democratic (WEIRD) populations that are typical of eating disorder research samples.

In our PPI consultation it was suggested that a high sense of clarity about one's feelings might be correlated with a sense of certainty deriving from the eating disorder, and perhaps an identification with the 'eating disorder voice' which is associated with markers of severity such as longer duration of illness (Pugh \& Waller, 2017). This perspective is congruent with the notion that ineffective mentalizing might influence outcome through lower levels of epistemic trust (Fonagy et al., 2015), resulting in reduced openness to learning in therapy. We speculate that the alliance scales predictive of positive outcome - Sense of Safety and Emotional Connection for the parents, and Shared Sense of Purpose for the adolescent - may represent preconditions or correlates of epistemic trust. The association between outcome and the parental SOFTA Emotional Connection and Safety scales is in keeping with themes from qualitative studies which emphasise the importance for parents of trusting and feeling understood by their therapist (Wallis et al., 2017a; Williams et al., 2020). Wallis et al. (2017a) have described a therapeutic process in FT-AN that they term relational containment, whereby treatment factors including regular appointments, set treatment sequence and consistent support created a sense of stability for parents, which helped 
give them the confidence to approach their adolescent differently. This then helped to establish a virtuous circle whereby increased parental confidence helped their adolescent to feel more secure, thereby promoting positive change such as improved communication.

Our findings support the notion that the development of this sense of relational containment in the family may represent an important early treatment goal, which from the perspective of adolescents might include establishing a shared sense of purpose for therapy. Notably, secure attachment and higher reflective function in adolescents were both predictive of positive alliance ratings, suggesting that these variables merit inclusion in future studies of alliance formation in FT-AN, such as studies incorporating session-by-session data collection and observational measures of alliance.

\subsubsection{Limitations}

Our study has several limitations, such as the use of self-report methods for both our independent variables and hypothesised mediator, thereby raising the issue of singlemethod variance. Feedback from PPI activities highlighted that our choice of outcome was too reliant on weight as a criterion for success and did not include broader themes, such as positive indicators of well-being, which have been shown to be important to recovery from the perspective of those with lived experience (Bohrer et al., 2020). Thus, constraints in our definition of treatment outcome, and psychometric limitations of available measures of our exposure and mediator variables, constitute a source of imprecision in our study. The choice of nine months 
as the final time-point for the study was critiqued as too early in treatment, and longer follow up periods should be incorporated into future research designs. Finally, the lack of data on parental psychopathology is a further limitation, since such data could have shed light on alternative explanations of our findings. Morandotti et al. (2018) found RFQ Certainty scores to be positively correlated with self-reported anxiety and depression scores in their healthy control sample of 158 Italian adults. This raises the possibility that parents in our study with high scores on the RFQ Certainty scale may have higher levels of psychopathology, which could have impacted on their parenting in various ways, including the ability to think flexibly and problem-solve during FT-AN.

\subsection{Conclusion}

In summary, our data are the first to suggest that mentalizing difficulties may predict poor outcome in FT-AN, particularly when present in parents. Study strengths include the relatively large sample drawn from three different sites providing routine treatment, thereby increasing the external validity of the study. Other strengths include the use of PPI to inform both method and interpretation. Future research should use multi-method approaches to investigate processes of change, including the roles of mentalizing and alliance, in studies using broader definitions of recovery that are salient to parents and adolescents with lived experience of eating disorders. 
Chapter 6 - Recovery as a process: Exploring definitions of recovery in the context of eating-disorder-related social media forums

The following chapter presents research published in the following publication:

Bohrer, B., Foye, U. and Jewell, T. (2020). Recovery as defined by users of eatingdisorder-related social media forums. International Journal of Eating Disorders, 53 (8) 1219-1223. 


\subsection{Abstract}

Objective: Online forums related to eating disorders (EDs) represent sources of support for recovery, and comments on these platforms might therefore highlight aspects of recovery that have been previously neglected in research. Reddit, an online discussion platform, hosts several ED-related forums. Due to the unique benefits of examining ED-related social media comments, we aimed to use a qualitative approach to conduct an exploratory study to examine users' conceptualizations of recovery from an ED.

Method: We extracted public comments mentioning recovery that were posted on three ED-related online forums on Reddit between March 2017 and August 2017. We thematically analyzed the data corpus using an inductive approach to examine how recovery is defined in the context of ED-related online communities.

Results: Two superordinate themes ('Recovery as a Process,' 'Psychosocial Factors') and three subordinate themes emerged (within the 'Psychosocial Factors' theme: cognitive/affective, behavioral/physical, social).

Discussion: The data support a definition of recovery that includes positive aspects of well-being and quality of life. Furthermore, the data highlight that recovery is experienced as an ongoing process that is unique to each individual. 


\subsection{Introduction}

The eating disorder (ED) field lacks agreed definitions of recovery and remission (Bardone-Cone, Hunt and Watson, 2018). As a consequence, treatment studies applying different criteria have reported hugely variable rates; for instance, in the field of family therapy for adolescent anorexia nervosa (FT-AN), Le Grange et al. (2019) recently reported that remission rates across randomized controlled trials range from $21 \%$ to $96 \%$. Recent years have seen increasing research attention paid to the issue of defining recovery and remission in eating disorders (e.g. see reviews by BardoneCone, Hunt and Rowan, 2018; de Vos et al., 2017; Khalsa et al., 2017). As argued by Bardone-Cone, Hunt and Rowan (2018, p.2), without agreement on a definition of recovery and how to measure it, the field cannot: "meaningfully compare recovery rates across studies; make strong claims about relative treatment effectiveness; identify reliable predictors of recovery; and report meaningful relapse rates". The importance of the topic to the field is underscored by the decision to dedicate a special issue to it in the International Journal of Eating Disorders (Weissman, 2020). The present study was included in this special issue and investigates the issue of recovery definitions from the perspective of persons with lived experience of an ED.

\subsubsection{Distinguishing remission and recovery}

Recovery and remission are two terms that are often used interchangeably in the literature, and universally agreed definitions of either term are lacking (Bardone-Cone, Hunt and Rowan, 2018; Khalsa et al., 2017). Nevertheless, an attempt to distinguish the concepts of remission and recovery can be made with reference to two dimensions: time and breadth of concept. In their seminal paper on consensus definitions for remission, recovery, relapse and recurrence in depression, Frank et al. (1991) 
differentiated remission and recovery on the basis of time. Citing Frank et al. (1991), Couturier \& Lock (2006) define remission as an assessment at a single point in time indicating that symptoms are substantially no longer present, at least for a brief period of time. Kordy et al. (2002), also building on Frank et al.'s (1991) work, propose an operationalization of eating disorder remission and recovery whereby full remission can be assessed at three months, whereas recovery criteria are applied at 12 months. This distinction between remission and recovery based on time remains influential, and informed Wade \& Lock's (2020) recent work on developing consensus definitions within the membership of the Eating Disorders Research Society.

However, recovery and remission can also be differentiated in terms of the breadth of the construct. Remission is typically used to refer to the abatement of symptoms within a medical model of disease, which does not fully capture the experiences of personal recovery for individuals with lived experience of ED (Wetzler et al., 2020). By contrast, the mental health recovery movement (Anthony, 1993) proposes a much broader conceptualization of recovery that emphasises subjective experiences and meaning:

"Recovery is described as a deeply personal, unique process of changing one's attitudes, values, feelings, goals, skills, and/or roles. It is a way of living a satisfying, hopeful, and contributing life even with limitations caused by illness. Recovery involves the development of new meaning and purpose in one's life as one grows beyond the catastrophic effects of mental illness." (Anthony, 1993, p.527). 


\subsubsection{Defining recovery and remission in adolescent anorexia nervosa}

RCTS of FT-AN have not been consistent in the ways that outcomes have been defined (Le Grange et al., 2019), nor have they all used the same terminology to define outcome. Nevertheless, the term 'remission' - used to describe the primary outcome in numerous trials (e.g. Agras et al., 2014; Le Grange et al., 2016; Lock et al., 2010) is certainly appropriate on the basis of the focus on symptom improvement, rather than the broader conceptualization of recovery outlined above. Le Grange et al. (2019) provide a detailed overview of how definitions of remission have varied across the body of FT-AN trials, before then applying different definitions to data from the Le Grange et al. (2016) trial, thereby illustrating how widely estimates of remission vary dependent on the stringency of definitions.

To give just a thumbnail sketch of the field, it is possible to characterize definitions of remission in FT-AN in terms of two main categories. The first is the use of Morgan Russell scales (Morgan \& Russell, 1975), which take a categorical approach based on weight, menstrual status and the presence of bulimic symptoms (see Chapter 5 for more details on operationalization). FT-AN studies undertaken in the United Kingdom, including the main study of this thesis, have tended to use the Morgan Russell approach (e.g. Eisler et al., 2000, 2016; Russell et al., 1987). The second approach to defining remission has been to apply a weight criterion (e.g. $>95 \%$ expected body weight) and a cognitive symptom criterion (scores on the Eating Disorder Examination (Fairburn et al., 1993) within one standard deviation of community norms). This approach has been taken in a number of trials of Family Based Treatment (Lock et al., 2001) including those by Le Grange et al. (2016), Lock et al. (2010) and Madden et al. (2015). 


\section{$\underline{\text { 6.2.3 Perspectives on recovery from persons with lived experience }}$}

Recent efforts to build consensus on definitions of recovery in the ED field have confirmed the importance of weight and cognitive symptom domains as important, but have also brought attention to the need to include improvements in functioning in numerous other domains, including psycho-social ones (Wade \& Lock, 2020; Wetzler et al., 2020). Accurso et al. (2020) have made the point that highly focused definitions of recovery employing cut-offs for percentage body mass index or Eating Disorder Examination (Fairburn et al., 1993) scores may not represent what patients or caregivers believe is most important. A growing number of qualitative studies on ED recovery from the perspective of individuals with lived experience attest to the need to conceptualise recovery more broadly than symptom management. For example, in their recent systematic review and meta-synthesis of such studies, Wetzler et al. (2020) found the following themes to emerge in conceptualizations of recovery: supportive relationships, hope, identity, meaning and purpose, empowerment and selfcompassion. Thus, whilst it is crucial to develop a standardized definition of recovery, such a definition should also accurately capture the lived experiences of persons with EDs to ensure our models of understanding reflect patients' experiences and subjective meanings. Thorough examination of persons' qualitative experiences of ED recovery may provide valuable insight into facets of ED recovery that are missed by routine outcomes assessment.

\subsubsection{Social media and eating disorder recovery}

For some, social-media platforms represent sources of support for ED recovery (e.g., Aardoom et al., 2014), and comments on such platforms may therefore highlight aspects of recovery that have not been included in previous research. Social-media 
platforms are beginning to be used to further examine the concept of recovery by those in various stages of change. For example, Keski-Rahkonen and Tozzi (2005) conducted analyses of a Finnish-language ED-related forum and found that participants' concept of recovery changed in tandem with their stage of change. As such comments are unsolicited, they are not influenced by potential researcher biases (e.g. demand characteristics) thus providing an advantage to common researcherdirected qualitative techniques such as interviews and focus groups.

Reddit, one such social-media platform, hosts several ED-related forums ("subreddits") that have been the focus of several studies (e.g., Moessner et al., 2018). Given the unique benefits of examining ED-related social-media comments, we aimed to use a qualitative approach to conduct an exploratory study to examine users' conceptualizations of ED recovery.

\subsection{Method}

\subsubsection{Corpus Selection and Data Extraction}

We extracted the data corpus from a freely available archive of public comments made on Reddit (Complete Public Reddit Comments Corpus, 2018), and limited the data to a six-month period (March 2017 to August 2017). In line with a similar study that analyzed ED subreddits more generally (McCaig et al., 2019), we focused our analyses on a group of three recovery-focused EDR subreddits: r/eating_disorders, r/EatingDisorders, and r/fuckeatingdisorders. Using an existing list of five recovery terms ('recovery', 'recover', 'recovers', 'recovered', 'recovering'), we extracted all comments posted on the three subreddits that included at least one recovery term. This study was deemed exempt from requiring ethical approval by the King's College 
London ethical board, and we provided the Python code (Python Software Foundation, 2017) written for data extraction as Supplementary Material for the published paper (available at https://doi.org/10.1002/eat.23218).

\subsubsection{Data Analysis}

We analyzed the data corpus using inductive thematic analysis at a semantic level (Braun \& Clarke, 2006). U.F. and T.J. independently conducted a thematic analysis on the data corpus and then compared analyses to reach a consensus on the thematic structure. B.B. acted as a "critical friend" (Smith \& Sparkes, 2006) to encourage reflexivity in the thematic structure. We consulted Braun and Clarke's six-step guide (2006) throughout the analyses and used NVivo 12 software to complete the analyses. Instead of including exact quotations to support our findings, we paraphrased quotations to prevent identification of commenters through internet searches (cf. Williams et al., 2018).

\subsection{Results}

\subsubsection{User Characteristics}

In total, 294 commenters generated the data. Commenters self-defined their ED, with self-reported diagnoses of anorexia nervosa, bulimia nervosa, binge-eating disorder, and sub-clinical or non-diagnosed experiences included within the data. Period of recovery was tracked during analysis, and the majority of users self-reported they were 'in recovery,' while a smaller sample self-reported they were 'recovered' or actively not recovered or engaging in self-defined recovery efforts. 


\subsubsection{Data Corpus Characteristics}

Overall, 2,848 comments were posted in the three subreddits between March 2017 and August 2017; 505 (18\%) of the comments contained at least one recovery term and comprised the data corpus for the present study. Each commenter contributed a mean of two comments $(S D=2$; range $=1-22)$. Each comment contained a mean of 202 words $(S D=202 ;$ range $=14-1,996)$.

\section{$\underline{6.4 .3 \text { Themes }}$}

Two superordinate themes ('Recovery as a Process,' 'Psychosocial Factors') and three subordinate themes ("sub-themes" within the 'Psychosocial Factors' theme: cognitive/affective, behavioral/physical, social) were identified. The themes are described in Table 6.1. 
Table 6.1 Eating-disorder subreddit themes.

\begin{tabular}{|c|c|c|c|}
\hline Theme & Description & Sub-themes & Examples \\
\hline${ }^{1}$ Process & $\begin{array}{l}\text { - Recovery is a non-linear process, } \\
\text { including several steps, and is unique } \\
\text { to the individual. } \\
\text { - These steps can be positive/forwards } \\
\text { (e.g., positive choices, realizations), } \\
\text { or negative/backwards (e.g., relapse). } \\
\text { There is not always a definitive end to } \\
\text { the recovery process. }\end{array}$ & $\begin{array}{l}\text { - Recovery is a process } \\
\text { - Everyone's path to recovery is different } \\
\text { - Turning point (main) } \\
\text { - 'Choose life' } \\
\text { - Recovery is not linear; relapse is normal } \\
\text { - Tired of ED as motivator }\end{array}$ & $\begin{array}{l}\text { - 'struggle' } \\
\text { - 'long ass thing' } \\
\text { - 'exhausting' } \\
\text { - 'f***ing sucks' } \\
\text { - 'long' } \\
\text { - 'difficult' } \\
\text { - 'two years of switchbacks' } \\
\text { - 'it's a path' }\end{array}$ \\
\hline $\begin{array}{l}{ }^{2} \text { Cognitive } \\
\text { and } \\
\text { affective } \\
\text { factors }\end{array}$ & $\begin{array}{l}\text { - Cognitive and affective factors that } \\
\text { comprise, influence, and result from } \\
\text { recovery. }\end{array}$ & $\begin{array}{l}\text { - Commitment } \\
\text { - Challenging the ED (e.g., 'feelings aren't } \\
\text { facts') } \\
\text { - Reconnecting with one's body } \\
\text { - Connecting to painful emotions } \\
\text { - Coping differently } \\
\text { - Identity } \\
\text { - Improved emotional well-being } \\
\text { - Recovery = freedom }\end{array}$ & $\begin{array}{l}\text { - 'forgiveness' } \\
\text { - 'wanting to change' } \\
\text { - 'desire' } \\
\text { - 'commitment' } \\
\text { - 'have to want it' } \\
\text { - 'choice' }\end{array}$ \\
\hline $\begin{array}{l}{ }^{2} \text { Behavioral } \\
\text { and physical } \\
\text { factors }\end{array}$ & $\begin{array}{l}\text { - Behavioral and physical factors that } \\
\text { comprise, influence, and result from } \\
\text { recovery. }\end{array}$ & $\begin{array}{l}\text { - Challenging the ED (e.g., breaking habits) } \\
\text { - Reconnecting with one's body } \\
\text { - Better physical health } \\
\text { - Normalizing diet }\end{array}$ & $\begin{array}{l}\text { - 'focus on what my body can do' } \\
\text { - 'focus on body function instead of weight' }\end{array}$ \\
\hline $\begin{array}{l}{ }^{2} \text { Social } \\
\text { factors }\end{array}$ & $\begin{array}{l}\text { Social factors that comprise, } \\
\text { influence, and result from recovery. }\end{array}$ & $\begin{array}{l}\text { - Social connectedness } \\
\text { - Social connection }\end{array}$ & $\begin{array}{l}\text { - 'admitting to loved ones how bad it was' } \\
\text { - 'best thing is the closeness I feel with other } \\
\text { people' }\end{array}$ \\
\hline
\end{tabular}




\subsubsection{Recovery as a process}

A central theme was the conceptualization of recovery as a process. The majority of individuals described themselves as 'in recovery,' while a small number identified as 'recovered.' Irrespective of how individuals identified their recovery status, commenters used a range of terms including 'journey' and 'path' to describe their process of recovery. This process encapsulated an array of stages, rather than describing recovery as an end process - thus, recovery was about more than merely gaining/losing weight or abstaining from ED behaviors. Central to this theme for many commenters was that the recovery process was unique to them, their experiences, and their recovery goals, meaning there is no "one-size fits all" definition of recovery due to individual differences in each person's recovery journey.

In addition, commenters described stages of recovery which can be loosely represented by the Transtheoretical Model (Prochaska \& DiClemente, 1983), which has been used previously as a theoretical framework for ED recovery (KeskiRahkonen \& Tozzi, 2005; Bardone-Cone, 2012). Contemplation was at the core of the ED-related forum discussions with recovery being conceptualized as a 'choice' that resulted from an event or 'turning point' to the realization that the "benefits" of the ED no longer outweighed the negative physical, social, and/or mental outcomes. While the desire to 'choose life' over the ED was core to creating a need to change, there was a need for preparation and determination within the action stages of this change as commenters reflected that the reality of recovery was a long, exhausting and difficult path.' 
Several commenters noted their need to choose recovery every morning. Furthermore, commenters reflected that recovery was not merely an end-goal but instead was a 'constant process' that differs for each individual. Relapse during this process was an important theme, as many commenters noted that recovery is 'not a linear process' as it will have "ups and downs," as well as relapse, which was widely recognized as a normal and oftentimes necessary part of the recovery process.

\section{$\underline{6.4 .5}$ Psychosocial factors}

Three psychosocial factors comprised the second sub-theme, in which commenters described a range of positive aspects to recovery. Under the sub-theme of cognitive and affective factors, many commenters stated that recovery equated to an experience of 'freedom' from the negative effects of the ED, such as feelings of guilt, the compulsion to engage in rituals, and preoccupation with food, calories, and weight. Commenters described positive attributes of emotional well-being, such as 'happiness,' a sense of 'authenticity,' and connection to others, but also mentioned the ability to connect to painful emotions. Commenters spoke of coping differently in order to 'break the cycle' and create 'healthy thought processes.' Relatedly, many emphasized behavioral and physical factors, with a change towards regular eating being an often-cited part of the recovery process. Moreover, recovery was said to entail a state of positive physical health in which one's 'body is able to function properly.' Finally, social factors played an important role in descriptions of both the process and outcome of recovery. For instance, interactions with close others were reported by some as critical in providing the initial motivation to recover, and also in providing social support during recovery. Commenters also described increased social connectedness as a positive attribute of recovery, whereby the 'world opens up' and 
they may experience 'closeness to others.' Some comments pointed to the possible temporal order of social factors in the recovery process. For instance, one commenter shared how it was 'intense loneliness' that prompted them to seek help. Other commenters reported that increased energy associated with physical recovery made social interactions easier and more enjoyable.

\subsection{Discussion}

Our findings support and expand upon existing research on consumer perspectives on ED recovery. In keeping with findings from two meta-syntheses of qualitative studies (de Vos et al., 2017; Wetzler et al., 2020) commenters in our study highlighted the importance of including positive psychosocial indicators of well-being in recovery definitions. Our data lend support to the critique that the behavioral or psychological symptoms often at the focus of contemporary treatment models do not match the complexity of the recovery process. Furthermore, existing definitions of recovery have largely focused on behavioural, psychological, and physical markers of recovery, usually in a categorical way (e.g. Bardone-Cone et al., 2010). Our findings are supportive of an emerging consensus on the importance of a broader conceptualization of recovery, beyond weight status and physical symptoms. This study replicates themes identified in interview-based qualitative studies of recovered individuals (e.g. Dawson et al., 2014), thereby extending the validity of such findings, given the difference in sampling method, which includes unsolicited comments from socialmedia users who typically reported still being in the process of recovery. The findings are also in keeping with those of Kenny, Boyle and Lewis (2020), whose study of recovery-focused ED blogs also aimed to investigate how ED recovery is conceptualized from the perspective of individuals with lived experience. The authors 
identified numerous themes in common with the present study, including recovery as a complex and non-linear process, recovery as a choice, and a theme of 'isolation to connection'. Bloggers in their study described social isolation as characteristic of ED, whereas recovery was characterized by renewed relationships.

\subsubsection{Attachment, affiliation and eating disorder recovery}

In the context of this thesis, it is pertinent to note that social connection emerged as a theme in the data, given its conceptual overlap with attachment. Indeed, social connection is a common theme in qualitative studies of eating disorder recovery from the perspective of persons with lived experience. In their recent meta-synthesis of such studies, Wetzler et al. (2020) followed the approach outlined by de Vos et al. (2017) and calculated a 'frequency effect size,' which is the total number of studies containing the theme divided by the total number of studies. By this metric, the theme of 'connectedness and sense of belonging' emerged as the most common theme (along with hope) in such studies, with a frequency effect size of $85 \%$. The authors conceptualise the overarching theme of supportive relationships (comprised of support and encouragement from others; connectedness and sense of belonging; peer support) as one of six key components of personal recovery from EDs.

Dawson et al.'s (2014) qualitative study using narrative inquiry (Clandinin \& Connelly, 2000) illuminates the possible ways in which social connection plays a role in recovery. In their study of adult women who had recovered from chronic AN, participants described having been through a long phase in which they had been unready or unable to change. During this phase participants described feeling misunderstood by others, including health professionals but also close others such as 
family and friends, and this was perceived by the participants as a lack of empathy. However, participants described how they reached a 'tipping point' which preceded a new phase of active pursuit of recovery. This tipping point could be one moment, or several, and comprised several factors that aligned, including developing insight and an internal locus of control, externalizing the illness, and feeling understood by others. From the perspective of recent mentalization-based theory (Fonagy et al., 2015; Luyten et al., 2020), it could be said that the participants began to develop epistemic trust - a theme to be explored further in Chapter 7 of this thesis.

Finally, the relevance of social connection to recovery in adolescents with ED is highlighted by recent findings from Accurso et al. (2020), whose study investigated caregivers' perspectives on ED recovery using an online survey. Asked to endorse items from a list that they considered essential to their definition of recovery, $66 \%$ endorsed an item related to social functioning in terms of reduced withdrawal/isolation, and $57 \%$ endorsed an item related to being able to socialize with peers.

Thus, there is converging evidence to suggest that social connection to others is a key part of ED recovery. Whilst the theme of social connection is not synonymous with attachment, it can be argued to be part of the 'attachment/affiliation' construct within the 'Systems for Social Processes' domain of the Research Domain Criteria (Cuthbert, 2014). Wetzler et al. (2020) maintain that there is a need to develop a measure of personal recovery in EDs, whilst Wade and Lock (2019) have argued for further research to validate definitions of recovery and remission using a standardized battery of assessments. From the perspective of the COSMIN framework (Vet et al., 2011), 
the construct validity of ED recovery, as a multidimensional construct that is 'formed' of physical, cognitive, emotional and social recovery is increasingly well-established, with growing consensus emerging between clinicians, researchers and consumers on the key aspects. Social connectedness forms part of the construct, but whether it is best measured within a broader ED recovery measure, or a standalone measure, is a question in need of empirical research.

\subsubsection{Eating disorder recovery - universal or unique?}

Whilst the present findings add to a growing evidence base that can inform the development of a consensus definition of ED recovery for the field, the data also speak to important limitations of such an endeavor. This is because people with lived experience of EDs frequently emphasize the unique, individual nature of the recovery process, as in our findings, and also because the meaning of recovery itself appears to differ when self-assessed by persons with lived experience of EDs, as compared to research-based criteria (Slof-Op't Landt, Dingemans, de la Torre Y Rivas, \& van Furth, 2019). If the meaning of ED recovery varies between individuals and also across time (Keski-Rakkonen \& Tozzi, 2005), this has important implications for both practice and research. In treatment, the use of personalized goals would help to ensure that therapy is congruent with person-specific meanings of recovery. For research, further development and validation of patient-centered outcome measures would constitute one means to advance the agenda of personalized, recovery-focused care (Simpson et al., 2016). 


\section{$\underline{\text { 6.5.3 Implications for service provision }}$}

The findings also suggest that treatment services which employ a process-focused, rather than categorical, theory of recovery may be more congruent with the lived experiences of persons with EDs. A dimensional, process-focused approach to the definition and prognosis of recovery aligns with recent research that found dimensional modelling of ED pathology to better predict ED course and outcome over time (Forbush et al., 2018). From a research perspective, assessment of recovery using continuous measures would also be helpful given that research designs employing categorical approaches to outcome, such as the main study of this thesis, require large sample sizes. Given the long-term nature of ED recovery and the constraints of current access to treatment, it may be optimal to have professional support built into treatment frameworks longitudinally, with definition of "critical need" for services as the onset of relapse before the ED is more severe and potentially harder to treat. Supplementary access to care and ongoing support networks - such as moderated online forums - may be helpful between service utilization.

\section{$\underline{\text { 6.5.4 Limitations }}$}

Our study must be considered in light of its limitations. Our data-extraction method may have missed other comments that discussed recovery using a synonymous term (e.g. 'healed'). However, our aim was to focus on the definition of the general term 'recovery' on ED-related forum discussions and we reached saturation of themes. Furthermore, because of the nature of the data corpus and because not all users mentioned a specific diagnosis, we were unable to examine themes of recovery in specific ED diagnostic categories. 


\subsection{Conclusion and Future Directions}

In conclusion, our findings suggest recovery is an ongoing and multifaceted process. Longitudinal follow-up studies with qualitative research built into the design are warranted to understand predictors of long-term success and relapse and to understand empirically how recovery fluctuates over time. Interpretative phenomenological analyses to explore lived experiences of the recovery process represent worthwhile next steps toward understanding mechanisms that contribute to recovery maintenance and relapse. Studies investigating the process and meaning of ED recovery to adolescent patients are required to establish whether the themes identified in the adult literature are also perceived as relevant to younger individuals with eating disorders. Future research on ED recovery must also prioritise the diversity of samples, given that existing studies are predominantly of white, young adult women with anorexia nervosa who have accessed specialist ED treatment (Bardone-Cone et al., 2018). Understanding how the meaning and achievement of recovery is influenced by contexts including age, gender, race, culture and sexual orientation should be a priority, not least since this might draw attention to different resources and barriers to recovery for individuals. Finally, we recommend that persons with lived experience of EDs be key collaborators in future research efforts in this area, including the development of psychometrically robust measures of recovery that are consistent with themes identified in this study. 


\section{Chapter 7 - Discussion}

\subsection{Introduction and overview of the thesis}

The aim of this thesis was to investigate the potential value of the constructs of attachment and mentalization to improving our understanding of who benefits from FT-AN, and how the treatment works. In this chapter, findings from across the studies of this thesis will be integrated alongside new developments in theory and evidence in the wider fields of attachment and mentalization, with the aim of providing an overall assessment of where the evidence now points.

In the Background chapter of the thesis it was argued that FT-AN is a treatment approach that has not undertaken sufficient research to test specific models of treatment process. In Chapter 2 , we undertook a narrative review of the literature of family therapy for child and adolescent eating disorders (EDs), utilising the lens of common factors. We argued that common factors might play a powerful role in FTAN, with a particular focus on early change in treatment.

In $\underline{\text { Chapter 3 }}$, we undertook a systematic review of attachment and mentalization and their association with child and adolescent eating pathology. We found both insecure attachment and mentalizing difficulties to be correlates of self-reported eating pathology in children and adolescents. There was also evidence from longitudinal studies that attachment constitutes a risk factor for later eating pathology. Whilst studies with adolescent clinical eating disorder samples were in short supply at the time of the published study, the updated literature review found the number of such studies to be increasing. However, the roles played by attachment and mentalizing, in 
terms of predicting outcomes or treatment process for adolescents with EDs, remain largely unexplored.

In $\underline{\text { Chapter } 4}$ we reported a systematic review of the psychometric properties of attachment measures in middle childhood and adolescence. Such measures were found to have sub-optimal psychometric properties, regardless of assessment method. We argued that work is needed to more clearly define how attachment is conceptualised. We also argued that recent findings suggest a dimensional approach should be favoured over a categorical approach. Attachment may also best be conceived as a multidimensional construct. To develop useful measurement instruments, it may be helpful to develop measures of more precise, lower-order attachment constructs.

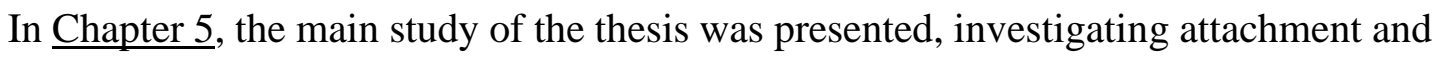
mentalization as predictors of outcome in FT-AN. A model was tested in which baseline attachment, mentalization and emotion regulation predicted outcome via the mediating variable of therapeutic alliance. Excessive certainty about mental states, particularly in parents, were found to predict poor outcome, but this was not mediated by alliance. Our hypothesised associations between attachment, mentalization, emotion regulation and therapeutic alliance were observed in the data, as were associations between alliance and outcome. We argued that the alliance scales that predicted outcome might represent correlates of epistemic trust and relational containment.

Chapter 6 presented a qualitative study of how eating disorder recovery is defined by users of social media recovery forums. Commenters on eating disorder recovery 
forums conceived of recovery as a journey or process in which relapse is seen as normal. Recovery was also seen as including positive indicators of mental well-being, such as a sense of freedom from the eating disorder, and greater social connection with close others. Themes related to attachment and social connection were reported in both the process and meaning of recovery - for instance, connecting with and deriving support from others was seen as part of the recovery process, whilst being able to experience and derive pleasure from closeness with others comprised one aspect of what recovery entails.

In this chapter, the findings of the research presented in this thesis will be synthesised and discussed in the light of recent evidence and theoretical developments in the field of attachment and mentalizing.

\subsection{Attachment and mentalizing - trait or state?}

Earlier in this thesis we introduced attachment and mentalizing as broad and multifaceted constructs, which are at their closest point theoretically in the developmental model developed by Fonagy et al. (2002). We noted the similarities between this model and Bruch's (1973) account of the development of the self in the context of the child's early attachment relationships. Within both accounts, misattuned caregiving in early life represents a risk factor for psychopathology in later developmental periods. However, Bruch also highlighted the impact of starvation on psychological functioning, which we argued has been borne out by subsequent research such as Bora and Köse's (2016) meta-analytic findings on theory of mind impairments in acute vs. recovered anorexia nervosa (see Chapter 3). We have also drawn attention to the instability of attachment over the life-course (Pinquart et al., 2013). However, as yet we have not considered the extent to which attachment and 
mentalizing, as operationalized and measured within this thesis, might be stable or subject to change, nor how they might be impacted by starvation in young people. Put differently: do attachment and mentalizing represent states or traits?

A related and important question is whether or not the measures used in the main study are able to capture the dynamic changes in mentalizing that are suggested by the switch-point model discussed in Chapter 5. Luyten et al. (2019), in their overview of the assessment of mentalizing, make the distinction between measures that can capture online, 'hot' mentalizing versus more offline, 'cold' self-report measures, arguing that the former have higher ecological validity in capturing mentalizing as it unfolds in real-life situations. Likewise, self-report attachment measures are more likely to tap 'colder,' more cognitive appraisals of attachment beliefs and behaviours, which might be more likely to be stable. In a rare study of attachment change over time in adolescent AN, Wallis et al. (2017b) found attachment to mothers and fathers to decrease between baseline and session 20 (approximately 14 months after baseline), and then remain unchanged between session 20 and 12-month follow-up. However, when they compared this adolescent AN group to a non-clinical sample, the decline in attachment security to mothers and fathers was found across both groups, suggesting that the decline could represent a normative shift rather than a particular phenomenon for adolescents with AN. These findings serve as a reminder that in seeking to answer the question of whether attachment and mentalization represent traits or states in adolescent AN, one must keep in mind how attachment and mentalization have been operationalised, and how the findings compare to community samples. In the context of our main study dataset in this thesis, there will be an opportunity to shed light on the question of change in 
attachment and mentalization across time, since participants completed a battery of self-report measures of attachment and mentalizing at nine months. This will be an important secondary analysis to conduct in the future.

\subsection{Common and specific factors in FT-AN}

In Chapter 2, we made two theoretical arguments about how FT-AN might work, invoking both common and specific factors. Firstly, arguing in favour of common factors, we suggested that components of treatment including the expertise of a specialist eating disorders team, and the provision by clinicians of a narrative relating to the impact of the eating disorder on family life, facilitate a context in which epistemic trust develops. Secondly, arguing for specific factors, we argued that attachment, mentalizing and emotion regulation might moderate the extent to which epistemic trust and alliance develop. In our main study, we then tested a specific model in which attachment and mentalizing predicted outcome via the mediating variable of therapeutic alliance. The findings of this study attest the importance of both specific and common factors in FT-AN.

On the one hand, we found excessive certainty (as measured by the RFQ8 Certainty scale in parents, and the DERS Lack of Clarity scale in adolescents) to be a predictor of poor outcome in FT-AN. As defined by Kazdin (2007, p.3), excessive certainty could also be described as a moderator, since it is a "characteristic that influences the direction or magnitude of the relation between the intervention and outcome". Nevertheless, given that the main study of this thesis did not employ an RCT design, it seems prudent to use the terminology of predictors or indicators of treatment response. To elaborate, RCTs can be considered the ideal conditions in which to 
investigate moderating effects, since the impact of a variable on outcome can be compared in two (or more) treatment conditions. As defined by Kraemer et al. (2002), if a variable such as age interacted with treatment condition then it could be defined as a moderator, whereas if the variable had a main effect without interaction then it would be defined as a non-specific predictor of treatment outcome. Thus, pending the investigation of constructs such as mental state certainty within RCT designs, we can only conclude that we have found evidence of a predictive effect, and cannot be sure of the specificity of this effect to treatment choice in adolescent AN. Theoretically, excessive certainty might represent a specific factor that is salient to mechanisms of change in FT-AN, but it was not correlated with our putative mediator, therapeutic alliance.

Where our data is more clearly in line with the common factors paradigm is in our findings on therapeutic alliance as a predictor of outcome, which is consonant with a large body of research in eating disorders (Graves et al., 2017). Whilst the association we found between alliance and outcome is far from novel, what is newer is the prospective association between secure attachment, greater reflective function, and less emotion regulation difficulties with higher ratings of therapeutic alliance. This supports our earlier formulation that such baseline variables might play a role in moderating the development of alliance and are therefore worthy of consideration as treatment targets. Moreover, whilst the exposure variables that predicted alliance did not also predict outcome, thus ruling out mediation as defined in the Baron and Kenny (1986) approach, one can still speak of these exposure variables as having an indirect effect on outcome via alliance (Hayes, 2009). 
One should also consider the limitations of our choice of outcome, which is both strongly determined by weight outcome, and carries with it a statistical penalty by virtue of its categorical rather than continuous status. Given that processes related to social connection to others appear relevant to the process and definition of outcome (see Chapter 6), one might speculate that our exposure variables may have shown associations with outcome had we operationalized the latter in a broader way. Additionally, had we chosen a continuous measure of outcome, this would have increased our statistical power. One further crucial limitation of the main study is that the analyses relied on the assumption of there being trends in the data at aggregate level, whereas a range of arguments have been put forward against using the collective as the unit of analysis in studies of mediation (Hofmann et al., 2020). Processes of change may in fact vary between individuals, and the relationships between variables may be complex, bi-directional and non-linear (Hofmann et al., 2020). For example, whilst we found several dimensions of therapeutic alliance at one month to predict outcome, there is little reason to think that a variable such as alliance should operate in a uniform way across all individuals (Cuijpers et al., 2019). Thus, whilst excessive certainty was not associated with self-reported alliance scores at one month, certainty may predict other facets of alliance not examined in this thesis, such as later alliance scores.

A lack of data on parental characteristics and psychopathology is another important limitation of the main study. As noted in Chapter 5, we have no data on parental psychopathology. In addition, we lack data on parental characteristics including variables such as expressed emotion and self-efficacy, which to-date have been the most widely researched parental constructs in the FT-AN literature. As a consequence, it is hard to integrate our findings with previous research on these constructs. 
Nevertheless, one can speculate that parental characteristics not measured in the study could have influenced the findings. For example, in a secondary analysis of the Lock et al. (2010) RCT comparing FBT with Adolescent Focused Therapy for adolescent AN, Forsberg et al. (2017) found lower percentage expected body weight at end of treatment for adolescents receiving FBT whose mothers scored above a cut-off for mild depression. As mentioned in Chapter 5, there is a positive correlation between RFQ Certainty scores and self-reported depression (Morandotti et al., 2018), thereby raising the possibility that parental depression could at least partly explain the relationship between parental mental state certainty and outcome. Theoretically, high levels of mental state certainty could be consistent with the subjective experience of depression and influence how respondents complete the measure; for instance, a person in the depths of depression might well strongly agree that "I always know what I feel". Had we collected data on parental depression then it would have been possible to include it as a covariate in our analyses, thereby shedding light on whether mental state certainty predicts outcome above and beyond any predictive effect conferred by depression.

Taken together, our findings appear to potentially speak to both common and specific factors in therapy, albeit with a range of limitations. However, during the past five years there have been a range of theoretical developments of relevance to this thesis. In the following section these are first outlined and then integrated with the findings of this thesis to propose possible ways forward for FT-AN research. 


\subsection{New developments in attachment and mentalization}

In earlier iterations of attachment theory, early childhood experiences with attachment figures were conceived as shaping the development of later personality and psychopathology, as well as influencing a person's ability to develop a 'secure base' from which to explore psychological material in therapy (e.g. Bowlby, 1988). However, recent years have seen new ideas emerge in the literature about the evolutionary purpose of attachment, and the primacy of its role in development, psychopathology and treatment process (e.g. Fonagy et al., 2017; Granqvist, 2020; Luyten et al., 2020). New empirical findings, which do not fit easily within earlier iterations of attachment theory, have been one driver change. Such findings include: relatively low longitudinal stability of attachment (Pinquart et al., 2013b); high heritability of adolescent attachment (Fearon et al., 2014); lower-than-expected associations between childhood attachment and developmental outcomes (e.g. Fearon et al., 2010); and concerns about the psychometric properties of measures assessing attachment (Fearon \& Roisman, 2017). Chapter 4 of this thesis (Jewell et al., 2019), in which the psychometric properties of attachment measures were systematically appraised, contributes to understanding of this latter issue, whilst also synthesising evidence pointing to the dimensional structure of attachment.

In addition to the difficulties raised by the above findings, there have also been theoretical developments, particularly in relation to evolutionary theory, that have spurred reconsideration of key tenets of attachment theory (Granqvist, 2020). Whereas Bowlby (1969) saw the evolutionary function of the attachment-behavioural system as protection, Granqvist (2020) has argued that Bowlby's evolutionary reasoning was 
unnecessarily narrow. Indeed, Bowlby's close collaborator Mary Ainsworth had suggested to Bowlby as early as 1965 , in private correspondence, that attachment might serve other functions, including training (Ainsworth, 1965).

In recent years, Fonagy and colleagues have outlined a theory in which the role of attachment as a context for social learning has been emphasised (e.g. Fonagy et al., 2015, 2017; Luyten et al., 2020). As discussed in several chapters of this thesis, a key notion in this work has been that of epistemic trust: the capacity to identify knowledge conveyed by others as personally relevant and generalizable to other contexts (Luyten et al., 2020). In brief, an important role of attachment relationships is hypothesised to be the transmission of learning, including the transmission of culture (Granqvist, 2020). Secure attachment is thought to facilitate this via the mechanism of epistemic trust, whereby attachment figures are assumed to be trustworthy and reliable sources of information. Fonagy and colleagues hypothesise that a common factor in psychological therapies, and a primary mechanism of change, is the opening up of the patient's potential for learning via epistemic trust in the therapist, thereby enabling openness to new learning, such as new skills or self-knowledge (Luyten et al., 2020). Importantly, this capacity for new learning is then thought to generalize beyond the treatment context, enabling patients to benefit and learn from positive influences in their social environment.

\subsection{Revisiting theory about mechanisms of change in FT-AN}

The research findings presented in this thesis can be integrated with the developments in theory outlined above to put forward a new formulation of the possible mechanisms of change in FT-AN. Fonagy et al. (2017a) have proposed that therapists can foster 
the development of epistemic trust early in treatment by providing to the patient a model for understanding their experiences that resonates with them personally, and by sensitively responding to the patient and 'mentalizing' them effectively. Applied to FT-AN, the first session of treatment, which includes the assessment, is an opportunity to make maximal use of this communication system as a spur to behavioural change and the fostering of hope. Fonagy et al.'s (2017) theoretical formulation is written primarily with individual therapies in mind, and its translation into family therapy requires some caveats, since it cannot be assumed that family therapists foster epistemic trust within all family members to an equal extent. Given the key role that parents play in supporting their adolescent to increase their intake in the early phase of treatment, it may be particularly important that parents develop trust in the treatment team and model within the first sessions. Yet the adolescent is by no means left out of this picture. For instance, Wallis et al.'s (2017a) notion of relational containment proposes that containment of the parents by the treating team also helps to provide a secure context for change for the adolescent. Moreover, positive ratings of alliance developed by adolescents to the nurse in the Parent-Focussed Treatment arm of Le Grange et al.'s (2016) trial were predictive of better outcome (Hughes et al., 2019b); this is striking given that the role of the nurse in the treatment was limited to weighing and spending 10 minutes speaking to the adolescent. One can speculate that even this relatively minimal therapeutic input (in terms of time) might be fostering the development of epistemic trust for the adolescent.

If we accept our suggestion (made in Chapter 5) that self-reported therapeutic alliance ratings might represent a correlate (or proxy) for epistemic trust, then the findings of the main study appear to support the notion that the development of epistemic trust is 
one component that helps to explain successful outcomes in FT-AN. Yet the identification of a variable associated with outcome is not the same as identification of the mechanism of change - that is, the processes or events that are responsible for the change (Kazdin, 2007). The theoretical assumptions underlying this thesis were that secure attachment, mentalizing strengths and better emotion regulation in families would help families to make positive changes; implicitly, it was assumed that the absence of these characteristics would be associated with lack of change or getting stuck. We proposed that the 'switch-point theory' explained in Chapter 5 might be the mechanism by which family members become overwhelmed, both in therapy and outside of it. Thus, affective over-arousal, leading to the emergence of pre-mentalizing modes of subjectivity, was postulated as preventing new learning and behavioural change. Whilst this pathway may well represent something of the treatment process for some families who experience poor outcomes, our data analysis - at the level of the collective - does not support this hypothesis. Instead, our data lends itself to a different formulation of the processes that might be implicated in poor treatment outcome. This new formulation is in keeping with and informed by new developments in theory and research over recent years.

\section{$\underline{\text { 7.5.1 Excessive certainty, rigidity and anorexia nervosa }}$}

In discussing our results in Chapter 5, we highlighted the importance of excessive certainty and speculated that this might explain poor outcome through rigidity or inflexibility, which might constrain problem-solving and the development of new patterns of behaviour. The notion of rigidity appears frequently in the literature on $\mathrm{AN}$, albeit the precise meaning of the term may differ between different authors working at different times. For instance, rigidity has featured in seminal clinical 
descriptions of individuals with AN and their families, including accounts by Bruch (1978) and Minuchin, Rosman and Baker (1978). Meanwhile, there is a large literature on cognitive flexibility in AN, which has recently been reviewed by Miles et al. (2020), who found no differences between adolescents with acute AN and healthy controls on neurocognitive tasks assessing cognitive flexibility, but did find differences between these groups in terms of self-reported flexibility. The authors draw attention to the need for research to establish the extent to which self-report and task-based measures are assessing the same construct.

Rigidity can also be understood as a trait related to autism spectrum disorder (ASD), which is known to be present in $\mathrm{AN}$ at raised prevalence rates compared with the general population (Westwood \& Tchanturia, 2017). Brede et al. (2020) recently developed a model of how AN develops and persists in individuals with autism, based on findings from their qualitative study of women with autism, parents of women with autism, and healthcare professionals. This model includes both direct and indirect pathways between autism-related difficulties and the development of restrictive eating: for instance, intense restricted interests in eating or exercise are postulated as constituting direct pathways. Meanwhile, restrictive eating might also arise as a coping mechanism in response to negative emotional consequences of autism-related difficulties, such as peer victimization, which constitutes an indirect pathway within the model. ASD traits including rigidity, intolerance of uncertainty and social difficulties can contribute to either direct or indirect pathways. Rigid thinking emerged in the thematic analysis as a variable that could both give rise to and maintain AN, for instance by making it harder to overcome eating disorder cognitions. 
Thus, the rigidity that clinicians such as Bruch and Minuchin observed in families of an individual with AN might be partly explained by the higher prevalence of ASD traits in AN, and the shared genetic pool influencing traits in families (Simic, Jewell and Eisler, 2020). The association between characteristics of ASD and longer illness duration might be explained by a lack of response to traditional treatments for AN patients with a profile of ASD traits, and also a circular process whereby unsuccessful treatment leads to a neurological 'scarring' effect, leading to more pronounced neuropsychological characteristics (such as rigidity), which then increases the likelihood of unsuccessful treatment (Saure et al., 2020). Regardless of the presence of ASD traits, it is important to bear in mind the negative impact of starvation on the capacity for social learning and neuropsychological functioning (Bora $\&$ K Köse, 2016). Indeed, one possible reason why baseline percentage median body mass index might be a consistent predictor of poor outcome, as replicated in our main study, could be the increased cognitive rigidity that follows starvation. Relatedly, the predictive power of longer duration of untreated illness, another predictor of poor outcome in my study, could also be influenced through the neuropsychological sequelae of starvation. Therefore, excessive mental state certainty in parents may be a marker for the presence of other factors, including autism traits, which might explain the longitudinal association with poor outcome. However, our findings are also open to much broader explanations.

\subsubsection{Rigidity as a common factor in psychopathology}

An alternative perspective on flexibility is suggested by recent theoretical work by Fonagy and colleagues, who suggest that lack of flexibility in higher order cognition represents a core aspect of vulnerability to psychopathology, and conversely that 
flexibility represents a core aspect of resilience (Fonagy et al., 2017a). Within this view, a function of higher order cognition is monitoring the efficiency of lower-order neural structures and their outputs; a failure of resilience occurs when "an individual is unable to change processing systems in a sufficiently flexible manner to maintain optimum outcome despite changed circumstances" (Fonagy et al., 2017, p.5). The authors give the example of a child whose perfectionism has served them well in the past but has become problematic during later stages of development. Such ideas can be readily applied to anorexia nervosa and the processes described earlier by Brede et al. (2020), in which patterns of behaviour and thinking can become increasingly entrenched. However, conceptualizing psychopathology as a failure of resilience is perhaps less useful in the treatment context than a theory about how to get out of stuck patterns.

\subsubsection{Social relationships and recovery}

As mentioned earlier in this chapter, Fonagy et al. (2017b) have drawn attention to the importance of generating epistemic trust beyond the therapy room. According to their argument, once the patient has (re)gained the capacity for social learning in the context of epistemic trust, and in the presence of an environment that is at least partly benign, the patient can also make use of other positive social experiences. Thus, the common factor driving change is not the acquisition of improved mentalizing per se, but the acquisition of the capacity for learning from social situations: as they put it, "the improved epistemic trust and abandonment of rigidity enables learning from experience once again" (Fonagy et al., 2017b). The findings reported in our qualitative

study (Chapter 6), and in the wider eating disorder recovery literature, attest to the importance of rekindling social connection to others. In addition, the recovery literature also suggests that this process that takes time; thus, attempts to investigate 
such a process via quantitative means possibly requires a time frame measured in intervals of years rather than months.

A key question is what variables might moderate processes of developing epistemic trust and learning from social experience? Luyten et al. (2020) have suggested that the p factor - a general factor of psychopathology (see Caspi \& Moffitt (2018) for a review) - might be one such variable. For instance, the liability to mental disorder may be related to a reduced (but potentially modifiable) capacity to make use of social relationships in ways that lead to 'virtuous cycles' of social connectedness, perhaps partly due to epistemic mistrust. By contrast, secure attachment and greater levels of reflective function may be associated with better social functioning and higher odds of developing epistemic trust with one's social network, as potentially supported by our findings of an indirect effect on treatment outcome of these variables via higher therapeutic alliance.

To recap, newer developments in attachment and mentalizing theory propose that interpersonal processes, including epistemic trust, provide a pathway for recovery through an improved capacity for learning from social experience. In the context of eating disorders, there is qualitative evidence from individuals with lived experience that interpersonal experiences do indeed provide a catalyst for recovery. For instance, Dawson et al. (2014) in their study of individuals who recovered from chronic anorexia nervosa, report that the 'tipping points' associated with starting to recover were associated with either improvements in their current relationships or establishing new and more helpful relationships. The theme of social connectedness also appears consistently in qualitative studies related to eating disorder recovery, as discussed in 
Chapter 6. Thus, the notion that social connectedness might play a role in eating disorder recovery is worthy of research, as is the notion of the p factor.

\subsubsection{Summary of proposed mechanisms of change in FT-AN}

We hypothesise that the development of epistemic trust and the acquisition of the capacity to learn from social experiences may constitute two generative mechanisms (Baron \& Kenny, 1986) accounting for therapeutic change in FT-AN. These overarching common factors may be moderated by baseline characteristics including attachment and mentalizing profile, including the presence of overly certain and inflexible thinking. Thus, both common and specific factors are considered important to change processes in FT-AN.

\subsection{Recommendations for future research}

\section{$\underline{\text { 7.6.1 Psychometric work }}$}

The findings of our systematic review of attachment measures raise important questions about the validity and reliability of measures of attachment in middle childhood and adolescence. However, the measurement of emotion regulation and mentalizing, the other key exposure variables in the main study, is also problematic. For example, Rice Warnell \& Redcay (2019) recently reported minimal correlations between theory of mind measures at various ages, including preschoolers, middle childhood and adulthood, raising doubts that theory of mind is a unitary construct. The need for improved measures of mentalizing has also been emphasised by Luyten et al. (2020). Meanwhile, measures of emotional dysregulation and negative affect are also in need of further research to clarify the distinctions between concepts and their measures (Juarascio et al., 2020). 
Psychometric work is needed to develop valid and reliable measures of the following constructs: attachment, mentalizing, emotion regulation, epistemic trust and eating disorder recovery. In terms of mentalizing measures, studies should investigate the construct validity of certainty/uncertainty and the hypo-/hypermentalizing dimensions in both adolescent and adult populations. Measures are needed that operationalize the concept of epistemic trust so that empirical research can be undertaken on this concept. Whilst measures of therapeutic alliance may serve as a proxy for epistemic trust, these concepts are not the same, thereby highlighting the need for distinct measures. In the context of FT-AN theory, the importance of the therapist to the family should decline towards the later phases of treatment (Eisler et al., 2016a), as the adolescent becomes increasingly independent and engaged in life beyond their illness. By contrast, the adolescent's capacity for learning from social experiences should remain high. Measurement approaches that may be appropriate aside from self-report include the use of observational studies using video-recorded sessions, and also the use of transcripts of therapy sessions.

\subsubsection{Better characterisation of families at risk of poor outcome}

Studies are needed of adolescents with AN and their parents/caregivers using diverse measures of attachment, mentalizing, emotion processing and autism spectrum disorder, utilising observational measures and experimental tasks alongside self-report questionnaires. Such studies should also include concepts that go beyond the "deficit model' of psychopathology and include measurement of strengths (Tchanturia et al., 2015). Whilst theory and research into negative affect has dominated eating disorder research, positive affect represents a promising avenue for research, with strong 
implications for treatment (Coniglio et al., 2019). In the main study of this thesis, very low scores on measures of emotion dysregulation - which in principle indicate an absence of emotion dysregulation difficulties - were predictive of poor outcome. Understanding the correlates of these low emotion dysregulation scores utilising other measures, including of positive affect and emotional expression, could help to develop a more nuanced understanding of what treatment targets to focus on in future research.

\subsubsection{Prospective observational studies of treatment outcome in FT-AN}

Prospective observational studies such as that conducted in Chapter 5 represent one avenue for testing theoretical models, including mediating processes. However, future studies can improve on our main study in several respects. Firstly, studies seeking to shed light on a broader conceptualization of recovery should include longer time intervals for follow-up of at least two years, based on both Public and Patient Involvement undertaken as part of this thesis, and underscored by recent evidence from caregivers of adolescent patients that full recovery from an eating disorder took up to four years from onset (Accurso et al., 2020). Studies of early change processes are worthwhile but there is also a need to test mediation models in which change processes take place over a longer time, meaning that putative mediators (such as epistemic trust) are measured at a time point such as six months into treatment. There is also a need for studies employing high-density data collection, with data points at short intervals, as advocated by Hofmann, Curtiss and Hayes (2020), in order to shed light on more complex, non-linear patterns of change. Such an approach could be employed to better understand change processes in the first one or two months of 
treatment and would be well suited to developing a better conceptualisation of the therapeutic alliance and its development.

\subsubsection{Experimental studies}

Cuijpers et al. (2019) have argued for the necessity of various lines of evidence in order to shed light on mechanisms of change. This requires taking a step beyond correlational associations between specific or common factors and outcome. The authors argue for evidence that includes temporal associations, dose-response relationships, multiple alternative potential mediators, theoretical models and experimental manipulation. An important issue raised by the main study of this thesis is whether excessive mental state certainty is amenable to change and represents a worthwhile treatment target. Notwithstanding the need for research on the psychometric properties of mentalizing measures, and greater understanding of the correlates of a construct such as mental state certainty, there is a need for studies that seek to manipulate certainty experimentally. For example, one could develop a treatment module that aimed to increase psychological flexibility, promote problemsolving and decrease psychological rigidity, drawing on Public and Patient Involvement to inform the format, length and timing of such a module. Such a module could initially be developed within the context of a single case experimental design (see Smith (2012) for a review); if results of such early work were promising, then the module could be evaluated within the context of an RCT. It is worth emphasising that such a trial would not have to be offered from the very start of treatment, but could be offered several months after it, perhaps offered to a more specific subgroup of families 
meeting criteria including lack of treatment progress and higher scores on measures of mental state certainty.

Another form of experimental study would be an RCT comparing CBT-E with FTAN. This would provide a valuable context for investigating common and specific factors. For example, exposure and habituation to feared food and its consumption may constitute common mechanisms in either treatment approach (Dalle Grave et al., 2019). However, the two treatments may differ in terms of other putative mechanisms: for instance, an increase in parental self-efficacy might mediate weight outcomes in FT-AN but not CBT-E.

\subsection{Conclusion}

This thesis aimed to advance the theoretical and empirical base for treatment processes in FT-AN by investigating the concepts of attachment and mentalization. Both constructs were found to be associated with child and adolescent eating pathology, with evidence accumulating in recent years. However, our systematic review found that attachment measures in middle childhood and adolescence typically lack evidence of adequate psychometric properties. In the main study, attachment predicted therapeutic alliance, but did not predict outcome. Excessive mental state certainty, a facet of mentalizing, predicted poor outcome but not alliance. Meanwhile, therapeutic alliance predicted outcome in ways that were linked theoretically to the notions of relational containment and epistemic trust. Finally, the qualitative study of recovery definitions in individuals with lived experience of eating disorders suggested that social connection, a concept related to the broader themes of attachment and affiliation, is important to both the process and meaning of eating disorder recovery. 
Future studies should investigate attachment, mentalization, therapeutic alliance and epistemic trust in FT-AN over longer time periods, using broader definitions of eating disorder recovery, and with improved and diverse measurement approaches. 


\section{References}

Aardoom, J. J., Dingemans, A. E., Boogaard, L. H., \& Van Furth, E. F. (2014). Internet and patient empowerment in individuals with symptoms of an eating disorder: A cross-sectional investigation of a pro-recovery focused e-community. Eating Behaviors, 15, 350-356. https://doi.org/10.1016/j.eatbeh.2014.04.003

Abell, F., Happé, F., \& Frith, U. (2000). Do triangles play tricks? Attribution of mental states to animated shapes in normal and abnormal development. Cognitive Development, 15(1), 1-16. https://doi.org/10.1016/S0885-2014(00)00014-9

Accurso, E. C., Sim, L., Muhlheim, L., \& Lebow, J. (2020). Parents know best: Caregiver perspectives on eating disorder recovery. International Journal of Eating Disorders, 53(8), 1252-1260. https://doi.org/10.1002/eat.23200

Agras, W. S., Lock, J., Brandt, H., Bryson, S. W., Dodge, E., Halmi, K. A., Jo, B., Johnson, C., Kaye, W., \& Wilfley, D. (2014). Comparison of 2 family therapies for adolescent anorexia nervosa: A randomized parallel trial. JAMA Psychiatry, 71(11), 1279-1286.

Ainsworth, M. (1965, October 7). Letter to John Bowlby. Mary Ainsworth papers, box M3168, folder 2. London, UK.

Ainsworth, M. D. S., Blehar, M. C., Waters, E., \& Wall, S. N. (1978). Patterns of Attachment: A Psychological Study of the Strange Situation. Erlbaum.

Ainsworth, M., \& Bell, S. M. (1969). Some contemporary patterns of mother-infant interaction in the feeding situation. In A. Ambrose (Ed.), Stimulation in early infancy (pp. 133-170). Academic Press.

Allen, J. G., Fonagy, P., \& Bateman, A. W. (2008). Mentalizing in Clinical Practice. American Psychiatric Pub. 
Allen, J. P., \& Miga, E. M. (2010). Attachment in adolescence: A move to the level of emotion regulation. Journal of Social and Personal Relationships, 27(2), 181190. https://doi.org/10.1177/0265407509360898

American Psychiatric Association. (2006). Treatment of patients with eating disorders. 3rd ed. American Psychiatric Association. American Journal of Psychiatry, 163 (Suppl):4-54.

American Psychiatric Association. (2013). The diagnostic and statistical manual of mental disorders: DSM-5 (Fifth ed.). American Psychiatric Association.

American Psychiatric Association. (1994) Diagnostic and Statistical Manual of Mental Disorders (4th edition) (DSM-IV). American Psychiatric Association.

Anthony, W. A. (1993). Recovery from mental illness: The guiding vision of the mental health service system in the 1990s. Psychosocial Rehabilitation Journal, 16(4), 11.

Apostolidou, E. (2019). Attachment in individuals with eating disorders compared to community controls: A systematic review and meta-analysis. King's College London.

Apostolidou, E., Yorke, I., Tahta-Wraith, K., Liston, S., Simic, M., Eisler, I., Fonagy, P., \& Jewell, T. (Manuscript in preparation). Attachment in individuals with Eating Disorders compared to Community Controls: A Systematic Review and Meta-analysis.

Arcelus, J., Mitchell, A. J., Wales, J., \& Nielsen, S. (2011). Mortality Rates in Patients With Anorexia Nervosa and Other Eating Disorders: A Meta-analysis of 36 Studies. Archives of General Psychiatry, 68(7), 724-731. https://doi.org/10.1001/archgenpsychiatry.2011.74 
Armsden, G. C., \& Greenberg, M. T. (1987). The inventory of parent and peer attachment: Individual differences and their relationship to psychological wellbeing in adolescence. Journal of Youth and Adolescence, 16(5), 427-454. https://doi.org/10.1007/BF02202939

Armstrong, J. G., \& Roth, D. M. (1989). Attachment and separation difficulties in eating disorders: A preliminary investigation. International Journal of Eating Disorders, $\quad 8(2), \quad$ 141-155. $\quad$ https://doi.org/10.1002/1098$\underline{108 X(198903) 8: 2<141:: A I D-E A T 2260080203>3.0 . C O ; 2-E}$

Asay, T. P., \& Lambert, M. J. (1999). The empirical case for the common factors in therapy: Quantitative findings. In M. A. Hubble, B. L. Duncan, \& S. D. Miller (Eds.), The Heart and Soul of Change (pp. 23-55). American Psychological Association.

Asen, E., \& Scholz, M. (2010). Multi-Family therapy: Concepts and techniques. Routledge.

Attili, G., Toni, A., Pentima, L. D., \& Roazzi, A. (2018). High anxiety attachment in eating disorders: Intergenerational transmission by mothers and fathers. Paideia, 28(Armstrong, D. M., Roth, J. G. (1989). Attachment and separation difficulties in eating disorders: A preliminary investigation. International Journal of Eating Disorders, 8, 2, 141-155. http://dx.doi.org/10.1002/1098108X(198903)8:2<141::AID-EAT2260080203>3.0.CO;2-E).

Bäck, E. A. (2011). Effects of Parental Relations and Upbringing in Troubled Adolescent Eating Behaviors. Eating Disorders, 19(5), 403-424. https://doi.org/10.1080/10640266.2011.609091 
Bardone-Cone, A. M. (2012). Examining the match between assessed eating disorder recovery and subjective sense of recovery: Preliminary findings. European Eating Disorders Review, 20, 246-249. https://doi.org/10.1002/erv.1123

Bardone-Cone, A. M., Harney, M. B., Maldonado, C. R., Lawson, M. A., Robinson, D. P., Smith, R., \& Tosh, A. (2010). Defining recovery from an eating disorder: Conceptualization, validation, and examination of psychosocial functioning and psychiatric comorbidity. Behaviour Research and Therapy, 48, 194-202. https://doi.org/10.1016/j.brat.2009.11.001

Bardone-Cone, A. M., Hunt, R. A., \& Watson, H. J. (2018). An Overview of Conceptualizations of Eating Disorder Recovery, Recent Findings, and Future Directions. Current Psychiatry Reports, 20(9), 79. https://doi.org/10.1007/s11920-018-0932-9

Baron, R. M., \& Kenny, D. A. (1986). The moderator-mediator variable distinction in social psychological research: Conceptual, strategic, and statistical considerations. Journal of Personality and Social Psychology, 51(6), 1173(6), $1173-1182$.

Baron-Cohen, S., Wheelwright, S., Hill, J., Raste, Y., \& Plumb, I. (2001). The "Reading the Mind in the Eyes" Test Revised Version: A Study with Normal Adults, and Adults with Asperger Syndrome or High-functioning Autism. The Journal of Child Psychology and Psychiatry and Allied Disciplines, 42(2), 241-251. https://doi.org/10.1017/S0021963001006643

Barone, L., \& Guiducci, V. (2009). Mental representations of attachment in eating disorders: A pilot study using the Adult Attachment Interview. Attachment \& Human Development, $\quad$ 11(4), 405-417. https://doi.org/10.1080/14616730902814770 
Bartholomew, K., \& Horowitz, L. M. (1991). Attachment styles among young adults: A test of a four-category model. Journal of Personality and Social Psychology, 61(2), 226-244. https://doi.org/10.1037//0022-3514.61.2.226

Bateman, A., \& Fonagy, P. (1999). Effectiveness of Partial Hospitalization in the Treatment of Borderline Personality Disorder: A Randomized Controlled Trial. American Journal of Psychiatry, 156(10), 1563-1569. https://doi.org/10.1176/ajp.156.10.1563

Batista, M., Žigić Antić, L., Žaja, O., Jakovina, T., \& Begovac, I. (2018). Predictors of eating disorder risk in anorexia nervosa adolescents. Acta Clinica Croatica, 57(3), 399-410. https://doi.org/10.20471/acc.2018.57.03.01

Baudinet, J., Simic, M., Griffiths, H., Donnelly, C., Stewart, C., \& Goddard, E. (2020). Targeting maladaptive overcontrol with radically open dialectical behaviour therapy in a day programme for adolescents with restrictive eating disorders: An uncontrolled case series. Journal of Eating Disorders, 8(1), 68. https://doi.org/10.1186/s40337-020-00338-9

Bentz, M., Jepsen, J. R. M., Pedersen, T., Bulik, C. M., Pedersen, L., Pagsberg, A. K., \& Plessen, K. J. (2017). Impairment of Social Function in Young Females With Recent-Onset Anorexia Nervosa and Recovered Individuals. Journal of Adolescent Health, 60(1), 23-32. https://doi.org/10.1016/j.jadohealth.2016.08.011

Berg, K. C., Peterson, C. B., Frazier, P., \& Crow, S. J. (2012). Psychometric evaluation of the eating disorder examination and eating disorder examinationquestionnaire: A systematic review of the literature. International Journal of Eating Disorders, 45(3), 428-438. https://doi.org/10.1002 
Bernecker, S. L., Levy, K. N., \& Ellison, W. D. (2014). A meta-analysis of the relation between patient adult attachment style and the working alliance. Psychotherapy Research, 24(1), 12-24. https://doi.org/10.1080/10503307.2013.809561

Biehl, M., Matsumoto, D., Ekman, P., Hearn, V., Heider, K., Kudoh, T., \& Ton, V. (1997). Matsumoto and Ekman's Japanese and Caucasian Facial Expressions of Emotion (JACFEE): Reliability data and cross-national differences. Journal of Nonverbal Behavior, 21(1), 3-21. https://doi.org/10.1023/A:1024902500935

Blakemore, S. J. (2008). The social brain in adolescence. Nature Reviews. Neuroscience, 9(4), 267-277. https://doi.org/10.1038/nrn2353

Blatt, S. J., Bers, S. A., \& Schaffer, C. E. (1993). The assessment of self descriptions. Unpublished manuscript, Yale University.

Bleiberg, E. (2013). Mentalizing-based treatment with adolescents and families. Child and Adolescent Psychiatric Clinics of North America, 22(2), 295-330. https://doi.org/10.1016/j.chc.2013.01.001

Blessitt, E., Voulgari, S., \& Eisler, I. (2015). Family therapy for adolescent anorexia nervosa. Current Opinion in Psychiatry, 28(6), 455-460.

Bohrer, B. K., Foye, U., \& Jewell, T. (2020). Recovery as a process: Exploring definitions of recovery in the context of eating-disorder-related social media forums. International Journal of Eating Disorders, 53, 1219-1223. https://doi.org/10.1002/eat.23218

Boone, L. (2013). Are attachment styles differentially related to interpersonal perfectionism and binge eating symptoms? Personality and Individual Differences, 54(8), 931-935. https://doi.org/10.1016/j.paid.2013.01.006 
Bora, E., \& Köse, S. (2016). Meta-analysis of theory of mind in anorexia nervosa and bulimia nervosa: A specific İmpairment of cognitive perspective taking in anorexia nervosa? International Journal of Eating Disorders, 49(8), 739-740. https://doi.org/10.1002/eat.22572

Borelli, J. L., Somers, J., West, J. L., Coffey, J. K., Los Reyes, A., \& Shmueli-Goetz, Y. (2016). Associations between attachment narratives and self-report measures of attachment in middle childhood: Extending evidence for the validity of the Child Attachment Interview. Journal of Child and Family Studies, 25, 1235-1246.

Bosmans, G., \& Kerns, K. A. (2015). Attachment in middle childhood: Progress and prospects. In G. Bosmans \& K. A. Kerns (Eds.), Attachment in middle childhood: Theoretical advances an new directions in an emerging field. New Directions for Child and Adolescent Development, 148, 1-14.

Bouchard, M.-A., Target, M., Lecours, S., Fonagy, P., Tremblay, L.-M., Schachter, A., \& Stein, H. (2008). Mentalization in adult attachment narratives: Reflective functioning, mental states, and affect elaboration compared. Psychoanalytic Psychology, 25(1), 47-66. https://doi.org/10.1037/0736-9735.25.1.47

Bourion-Bedes, S., Baumann, C., Kermarrec, S., Ligier, F., Feillet, F., Bonnmains, C., Guillemin, F. \& Kabuth, B. (2013). Prognostic value of early therapeutic alliance in weight recovery: A prospective cohort of 108 adolescents with anorexia nervosa. Journal of Adolescent Health, 52, 344-350.

Bowlby, J. (1969). Attachment and Loss: Attachment (Vol. 1). New York: Basic Books.

Bowlby, J. (1973). Attachment and Loss, Vol. 2: Separation: Anxiety and anger. New York: Basic Books. 
Bowlby, J. (1979). The Making and Breaking of Affectional Bonds. London: Tavistock.

Bowlby, J. (1980). Attachment and loss: Vol. 3: Loss. New York: Basic Books.

Bowlby, J. (1988). A Secure Base: Clinical Applications of Attachment Theory. New York: Taylor \& Francis.

Braun, V., \& Clarke, V. (2006). Using thematic analysis in psychology. Qualitative Research in Psychology, 3,77-101. https://doi.org/10.1191/1478088706qp063oa

Brede, J., Babb, C., Jones, C., Elliott, M., Zanker, C., Tchanturia, K., Serpell, L., Fox, J., \& Mandy, W. (2020). "For Me, the Anorexia is Just a Symptom, and the Cause is the Autism": Investigating Restrictive Eating Disorders in Autistic Women. Journal of Autism and Developmental Disorders.

Brennan, K. A., Clark, C. L., \& Shaver, P. R. (1998). Self-report measurement of adult attachment: An integrative overview. In J.A. Simpson. \& W.S. Rholes (Eds.), Attachment Theory and Close Relationships (pp. 46-76). The Guilford Press.

Brenning, K., Soenens, B., Braet, C., \& Bosmans, G. (2011). An adaptation of the Experiences in Close Relationships Scale-Revised for use with children and adolescents. Journal of Social and Personal Relationships, 28(8), 1048-1072. https://doi.org/10.1177/0265407511402418

Broberg, A. G., Hjalmers, I., \& Nevonen, L. (2001). Eating disorders, attachment and interpersonal difficulties: A comparison between 18- to 24-year-old patients and normal controls. European Eating Disorders Review, 9(6), 381-396. https://doi.org/10.1002/erv.421

Brochu, J. P., Meilleur, D., DiMeglio, G., Taddeo, D., Lavoie, E., Erdstein, J., Pauzé, R., Pesant, C., Thibault, I., \& Frappier, J.-Y. (2018). Adolescents' perceptions 
of the quality of interpersonal relationships and eating disorder symptom severity: The mediating role of low self-esteem and negative mood. Eating Disorders, 26(4), 388-406. https://doi.org/10.1080/10640266.2018.1454806

Bruch, H. (1973). Eating disorders: Obesity, anorexia nervosa, and the person within. Basic Books.

Bruch, H. (1978). The Golden Cage: The Enigma of Anorexia Nervosa. Harvard University Press.

Bruch, H. (1982). Anorexia nervosa: Therapy and theory. The American Journal of Psychiatry, 139(12), 1531-1538. https://doi.org/10.1176/ajp.139.12.1531

Brumariu, L. E., Kerns, K. A., \& Seibert, A. (2012). Mother-child attachment, emotion regulation, and anxiety symptoms in middle childhood. Personal Relationships, 19(3), 569-585.

Burge, D., Hammen, C., Davila, J., Daley, S. E., Paley, B., Lindberg, N., Herzberg, D., \& Rudolph, K. D. (1997). The relationship between attachment cognitions and psychological adjustment in late adolescent women. Development and Psychopathology, $\quad$ 9(1), 151-167. https://doi.org/10.1017/S0954579497001119

Byng-Hall, J. (1995). Rewriting family scripts: Improvisation and systems change. Guilford Press.

Byrne, C. E., Accurso, E. C., Arnow, K. D., Lock, J., \& Grange, D. L. (2015). An exploratory examination of patient and parental self-efficacy as predictors of weight gain in adolescents with anorexia nervosa. International Journal of Eating Disorders, 48(7), 883-888. https://doi.org/10.1002/eat.22376

Caglar-Nazali, H. P., Corfield, F., Cardi, V., Ambwani, S., Leppanen, J., Olabintan, O., Deriziotis, S., Hadjimichalis, A., Scognamiglio, P., Eshkevari, E., Micali, 
N., \& Treasure, J. (2014). A systematic review and meta-analysis of 'Systems for Social Processes' in eating disorders. Neuroscience \& Biobehavioral Reviews, 42, 55-92. https://doi.org/10.1016/j.neubiorev.2013.12.002

Caspi, A., \& Moffitt, T. E. (2018). All for One and One for All: Mental Disorders in One Dimension. American Journal of Psychiatry, 175(9), 831-844. https://doi.org/10.1176/appi.ajp.2018.17121383

Cassidy, J., Marvin, R. S., \& with the MacArthur Working Group. (1992). Attachment organization in preschool children: Procedures and coding manual. Unpublished manuscript, University of Virginia.

Cate, R., Khademi, M., Judd, P., \& Miller, H. (2013). Deficits in mentalization: A risk factor for future development of eating disorders among pre-adolescent girls. Advances in Eating Disorders, 1(3), 187-194. https://doi.org/10.1080/21662630.2013.794497

Child Growth Foundation. (1990). UK cross-sectional reference data: 1990/1. London: Child Growth Foundation. Child Growth Foundation.

Chorpita, B. F., Moffitt, C. E., \& Gray, J. (2005). Psychometric properties of the Revised Child Anxiety and Depression Scale in a clinical sample. Behaviour Research and Therapy, 33(3), 309-322. https://doi.org/10.1016/j.brat.2004.02.004

Clandinin, D. J., \& Connelly, F. M. (2000). Narrative inquiry. Jossey-Bass.

Cole-Detke, H., \& Kobak, R. (1996). Attachment processes in eating disorder and depression. Journal of Consulting and Clinical Psychology, 64(2), 282-290. https://doi.org/10.1037//0022-006x.64.2.282

Colton, P. A., Olmsted, M. P., Daneman, D., Rydall, A. C., \& Rodin, G. M. (2007). Natural history and predictors of disturbed eating behaviour in girls with Type 
1 diabetes. Diabetic Medicine: A Journal of the British Diabetic Association, 24(4), 424-429. https://doi.org/10.1111/j.1464-5491.2007.02099.x

Complete Public Reddit Comments Corpus 2018. Retrieved from https://archive.org/details/2015_reddit_comments_corpus.

Coniglio, K. A., Christensen, K. A., Haynos, A. F., Rienecke, R. D., \& Selby, E. A. (2019). The posited effect of positive affect in anorexia nervosa: Advocating for a forgotten piece of a puzzling disease. International Journal of Eating Disorders, 52(9), 971-976. https://doi.org/10.1002/eat.23147

Connan, F., Campbell, I. C., Katzman, M., Lightman, S. L., \& Treasure, J. (2003). A neurodevelopmental model for anorexia nervosa. Physiology \& Behavior, 79(1), 13-24. https://doi.org/10.1016/s0031-9384(03)00101-X

Cortés-García, Laura, Takkouche, B., Seoane, G., \& Senra, C. (2019a). Mediators linking insecure attachment to eating symptoms: A systematic review and meta-analysis. PLOS ONE, 14(3), e0213099. https://doi.org/10.1371/journal.pone.0213099

Cortés-García, L., Hoffmann, S., Warschburger, P., \& Senra, C. (2019b). Exploring the reciprocal relationships between adolescents' perceptions of parental and peer attachment and disordered eating: A multiwave cross-lagged panel analysis. International Journal of Eating Disorders, 52(8), 924-934. https://doi.org/10.1002/eat.23086

Cortés-García, L., Viddal, K. R., Wichstrøm, L., \& Senra, C. (2020a). Mediating role of depressive symptoms linking insecure attachment and disordered eating in adolescents: A multiwave longitudinal study. Development and Psychopathology, 1-13. https://doi.org/10.1017/S0954579420001029 
Cortés-García, L., McLaren, V., Vanwoerden, S., \& Sharp, C. (2020b). Attachment, mentalizing, and eating disorder symptoms in adolescent psychiatric inpatients and healthy controls: A test of a mediational model. Eating and Weight Disorders - Studies on Anorexia, Bulimia and Obesity. https://doi.org/10.1007/s40519-020-01017-z

Couturier, J., Isserlin, L., \& Lock, J. (2010). Family-based treatment for adolescents with anorexia nervosa: A dissemination study. Eating Disorders, 18, 199-209.

Couturier, J., Isserlin, L., Norris, M., Spettigue, W., Brouwers, M., Kimber, M., McVey, G., Webb, C., Findlay, S., Bhatnagar, N., Snelgrove, N., Ritsma, A., Preskow, W., Miller, C., Coelho, J., Boachie, A., Steinegger, C., Loewen, R., Loewen, T., ... Pilon, D. (2020). Canadian practice guidelines for the treatment of children and adolescents with eating disorders. Journal of Eating Disorders, 8(1), 4. https://doi.org/10.1186/s40337-020-0277-8

Couturier, J., Kimber, M., \& Szatmari, P. (2013). Efficacy of family-based treatment for adolescents with eating disorders: A systematic review and meta-analysis. International Journal of Eating Disorders, 46(1), 3-11. https://doi.org/10.1002/eat.22042

Couturier, J., Kimber, M., Jack, S., Niccols, A., Van Blyderveen, S., \& McVey, G. (2013). Understanding the uptake of family based treatment for adolescents with anorexia nervosa: Therapist perspectives. International Journal of Eating Disorders, 46, 177-188.

Couturier, J., Kimber, M., Jack, S., Niccols, A., Van Blyderveen, S., \& McVey, G. (2014). Using a knowledge transfer framework to identify factors facilitating implementation of family-based treatment. International Journal of Eating Disorders, 47, 410-417. 
Couturier, J.L. \& Kimber, M.S. (2015). Dissemination and Implementation of Manualized Family-Based Treatment: A Systematic Review. Eating Disorders, 23, 4, 281-290.

Couturier, J., \& Lock, J. (2006). What is Recovery in Adolescent Anorexia Nervosa? International Journal of Eating Disorders, 39(7), 550-555. https://doi.org/10.1002/eat.20309

Craig, P., Dieppe, P., Macintyre, S., Michie, S., Nazareth, I., \& Petticrew, M. (2008). Developing and evaluating complex interventions: The new Medical Research Council guidance. BMJ, 337. https://doi.org/10.1136/bmj.a1655

Crisp, A. H., Norton, K., Gowers, S., Halek, C., Bowyer, C., Yeldham, D., Levett, G., \& Bhat, A. (1991). A controlled study of the effect of therapies aimed at adolescent and family psychopathology in anorexia nervosa. The British Journal of Psychiatry, 159, 325-333. https://doi.org/10.1192/bjp.159.3.325

Crittenden, P. M., \& Dallos, R. (2009). All in the Family: Integrating Attachment and Family Systems Theories. Clinical Child Psychology and Psychiatry, 14(3), 389-409. https://doi.org/10.1177/1359104509104048

Crowell, J.A., Fraley, R.C., \& Roisman, G.I. (2016). Measurement of Individual Differences in Adult Attachment. In J. Cassidy \& P. R. Shaver (Eds.), Handbook of Attachment: Theory, research, and clinical applications (3rd ed., pp. 598-635). Guilford Press.

Cuijpers, P., Reijnders, M., \& Huibers, M. J. H. (2019). The Role of Common Factors in Psychotherapy Outcomes. Annual Review of Clinical Psychology, 15(1), 207-231. https://doi.org/10.1146/annurev-clinpsy-050718-095424 
Cuthbert, B. N. (2014). The RDoC framework: Facilitating transition from ICD/DSM to dimensional approaches that integrate neuroscience and psychopathology. World Psychiatry, 13(1), 28-35. https://doi.org/10.1002/wps.20087

Dalle G., R., \& Calugi, S. (2020). Cognitive Behavior Therapy for Adolescents with Eating Disorders (1st edition). Guilford Press.

Dalle G., R., Calugi, S., Doll, H.A. \& Fairburn, C.G. (2013). Enhanced cognitive behaviour therapy for adolescents with anorexia nervosa: an alternative to family therapy? Behaviour Research and Therapy, 51(1), R9-R12.

Dalle G., R., Eckhardt, S., Calugi, S., \& Le Grange, D. (2019). A conceptual comparison of family-based treatment and enhanced cognitive behavior therapy in the treatment of adolescents with eating disorders. Journal of Eating Disorders, 7(1), 42. https://doi.org/10.1186/s40337-019-0275-X

Dallos, R. (2004). Attachment narrative therapy: Integrating ideas from narrative and attachment theory in systemic family therapy with eating disorders. Journal of Family Therapy, 26(1), 40-65. $\quad$ https://doi.org/10.1111/j.1467$\underline{6427.2004 .00266 . x}$

Dallos, R. (2006). Attachment narrative therapy. McGraw-Hill Education (UK).

Dallos, R., \& Denford, S. (2008). A qualitative exploration of relationship and attachment themes in families with an eating disorder. Clinical Child Psychology and Psychiatry, 13(2), 305-322.

Dare, C., \& Eisler, I. (1997). Family Therapy for Anorexia Nervosa. In D. M. Garner \& P. E. Garfinkel (Eds.), Handbook of Treatment for Eating Disorders (2nd ed., pp. 307-324). The Guilford Press. 
Dare, C., Eisler, I., Colahan, M., Crowther, C., Senior, R., \& Asen, E. (1995). The listening heart and the chi square: Clinical and empirical perceptions in the family therapy of anorexia nervosa. Journal of Family Therapy, 17, 31-57.

Dare, C., Eisler, I., Russell, G., Treasure, J., \& Dodge, L. (2001). Psychological therapies for adults with anorexia nervosa: Randomised controlled trial of outpatient treatments. British Journal of Psychiatry, 178(3), 216-221. https://doi.org/10.1192/bjp.178.3.216

Dawson, L., Rhodes, P., \& Touyz, S. (2014). "Doing the Impossible": The Process of Recovery From Chronic Anorexia Nervosa. Qualitative Health Research, 24(4), 494-505. https://doi.org/10.1177/1049732314524029

De Vet, H.C., Terwee, C.B., Mokkink, L.B. and Knol, D.L., 2011. Measurement in Medicine. Cambridge University Press.

de Vos, J. A., LaMarre, A., Radstaak, M., Bijkerk, C. A., Bohlmeijer, E. T., \& Westerhof, G. J. (2017). Identifying fundamental criteria for eating disorder recovery: A systematic review and qualitative meta-analysis. Journal of Eating Disorders, 5(1), 34. https://doi.org/10.1186/s40337-017-0164-0

Decaluwe, V., \& Braet, C. (1999). Dutch translation of the Child Eating Disorder Examination, authored by C. G. Fairburn, Z. Cooper \& R. Bryant-Waugh. Unpublished manuscript.

Decaluwé, V., \& Braet, C. (2004). Assessment of eating disorder psychopathology in obese children and adolescents: Interview versus self-report questionnaire. Behaviour Research and Therapy, 42(7), 799-811. https://doi.org/10.1016/j.brat.2003.07.008 
Del Giudice, M., Angeleri, R., \& Manera, V. (2009). The juvenile transition: A developmental switch point in human life history. Developmental Review, 29, $1-31$.

Diamond, G. S., Reis, B. F., Diamond, G. M., Siqueland, L., \& Isaacs, L. (2002). Attachment-Based Family Therapy for Depressed Adolescents: A Treatment Development Study. Journal of the American Academy of Child \& Adolescent Psychiatry, 41(10), 1190-1196. https://doi.org/10.1097/00004583$\underline{200210000-00008}$

Diamond, G. S., Wintersteen, M. B., Brown, G. K., Diamond, G. M., Gallop, R., Shelef, K., \& Levy, S. (2010). Attachment-based family therapy for adolescents with suicidal ideation: A randomized controlled trial. Journal of the American Academy of Child \& Adolescent Psychiatry, 49, 122-131.

Diamond, G.M., Diamond, G.S. \& Levy, S.A. (2014). Attachment-Based Family Therapy for Depressed Adolescents. American Psychological Association.

Dimitropoulos, G., Freeman, V. E., Lock, J. \& Le Grange, D. (2015), Clinician perspective on parental empowerment in family-based treatment for adolescent anorexia nervosa. Journal of Family Therapy. doi: 10.1111/1467-6427.12086

Downs, K.J. \& Blow, A.J. (2013). A substantive and methodological review of familybased treatment for eating disorders: the last 25 years of research. Journal of Family Therapy 35, S1, 3-28

Doyle, P. M., Grange, D. L., Loeb, K., Doyle, A. C., \& Crosby, R. D. (2010). Early response to family-based treatment for adolescent anorexia nervosa. International Journal of Eating Disorders, 43(7), 659-662. https://doi.org/10.1002/eat.20764 
Duclos, J., Dorard, G., Cook-Darzens, S., Curt, F., Faucher, S., Berthoz, S., Falissard, B., \& Godart, N. (2018). Predictive factors for outcome in adolescents with anorexia nervosa: To what extent does parental Expressed Emotion play a role? PLOS ONE, $\quad$ 13(7), 0196820. https://doi.org/10.1371/journal.pone.0196820

Duncan, T. K., Sebar, B., \& Lee, J. (2015). Reclamation of power and self: A metasynthesis exploring the process of recovery from anorexia nervosa. Advances in Eating Disorders: Theory, Research and Practice, 3,177-190. https://doi.org/10.1080/21662630.2014.978804

Dykas, M. J., Woodhouse, S. S., Cassidy, J., \& Waters, H. S. (2006). Narrative assessment of attachment representations: Links between secure base scripts and adolescent attachment. Attachment \& Human Development, 8(3), 221-240.

Dziobek, I., Fleck, S., Kalbe, E., Rogers, K., Hassenstab, J., Brand, M., Kessler, J., Woike, J. K., Wolf, O. T., \& Convit, A. (2006). Introducing MASC: A movie for the assessment of social cognition. Journal of Autism and Developmental Disorders, 36(5), 623-636. https://doi.org/10.1007/s10803-006-0107-0

Egan, S. J., Wade, T. D., \& Shafran, R. (2011). Perfectionism as a transdiagnostic process: A clinical review. Clinical Psychology Review, 31(2), 203-212. https://doi.org/10.1016/j.cpr.2010.04.009

Eisler, I. (2005). The empirical and theoretical base of family therapy and multiple family day therapy for adolescent anorexia nervosa. Journal of Family Therapy, 27(2), 104-131. https://doi.org/10.1111/j.1467-6427.2005.00303.x

Eisler, I., \& Szmukler, G. I. (1985). Social class as a confounding variable in the Eating Attitudes Test. Journal of Psychiatric Research, 19(2-3), 171-176. https://doi.org/10.1016/0022-3956(85)90014-7 
Eisler, I., Dare, C., Hodes, M., Russell, G., Dodge, E., \& Le Grange, D. (2000). Family therapy for adolescent anorexia nervosa: The results of a controlled comparison of two family interventions. Journal of Child Psychology and Psychiatry, 41(6), 727-736. https://doi.org/10.1111/1469-7610.00660

Eisler, I., Dare, C., Russell, G. F. M., Szmukler, G., le Grange, D., \& Dodge, E. (1997). Family and individual therapy in anorexia nervosa: A 5-year follow-up. Archives of General Psychiatry, 54(11), 1025-1030. https://doi.org/10.1001/archpsyc.1997.01830230063008

Eisler, I., Dodge, E., \& Wallis, A. (2016). What's new is old and what's old is new: The origins and evolution of eating disorders family therapy. In K. L. Loeb, Le Grange, Daniel, \& Lock, James (Eds.), Family Therapy for Adolescent Eating and Weight Disorders. (pp. 26-62). Routledge.

Eisler, I., Le Grange, D. \& Lock, J. (2015). Treating adolescents with eating disorders. In: T. Sexton, J. Lebow (Eds.). Handbook of Family Therapy (pp.387-406). $4^{\text {th }}$ Edition. Routledge.

Eisler, I., Le Grange, D., \& Lock, J. (2015). Family therapy for adolescent eating disorders. In T. Sexton \& J. Lewbow (Eds.), Handbook of Family Therapy (4th edition). Routledge.

Eisler, I., Simic, M., Blessitt, E., Dodge, E., \& Team. (2016a). Maudsley service manual for child and adolescent eating disorders (Revised). https://mccaed.slam.nhs.uk/wp-content/uploads/2019/11/Maudsley-Service-

\section{Manual-for-Child-and-Adolescent-Eating-Disorders-July-2016.pdf}

Eisler, I., Simic, M., Hodsoll, J., Asen, E., Berelowitz, M., Connan, F., Ellis, G., Hugo, P., Schmidt, U., Treasure, J., Yi, I., \& Landau, S. (2016b). A pragmatic randomised multi-centre trial of multifamily and single family therapy for 
adolescent anorexia nervosa. BMC Psychiatry, 16(1), 422. https://doi.org/10.1186/s12888-016-1129-6

Eisler, I., Simic, M., Russell, G. F. M., \& Dare, C. (2007). A randomised controlled treatment trial of two forms of family therapy in adolescent anorexia nervosa: A five-year follow-up. Journal of Child Psychology and Psychiatry, 48(6), 552-560. https://doi.org/10.1111/j.1469-7610.2007.01726.x

Eisler, I., Wallis, A. \& Dodge, E. (2015). What's new is old and what's old is new: the origins and evolution of family therapy for eating disorders. In K. Loeb, D. Le Grange \& J. Lock (Eds.). Family Therapy for Adolescent Eating and Weight Disorders (pp. 6-41). Routledge.

Ellison, R., Rhodes, P., Madden, S., Miskovic, J., Wallis, A., Kohn, M., \& Touyz, S. (2012). Essential components of the Maudsley model of family-based treatment for anorexia nervosa. International Journal of Eating Disorders, 45, $609-614$.

Engman-Bredvik, S., Suarez, N.V., Levi, R. \& Nilsson, K. (2015): Multi-Family Therapy in Anorexia Nervosa-A Qualitative Study of Parental Experiences. Eating Disorders, DOI: 10.1080/10640266.2015.1034053.

Epstein, N. B., Baldwin, L. M., \& Bishop, D. S. (1983). The McMaster Family Assessment Device. Journal of Marital and Family Therapy, 9(2), 171-180. https://doi.org/10.1111/j.1752-0606.1983.tb01497.x

Faber, A., Dubé, L., \& Knäuper, B. (2018). Attachment and eating: A meta-analytic review of the relevance of attachment for unhealthy and healthy eating behaviors in the general population. Appetite, 123, 410-438. https://doi.org/10.1016/j.appet.2017.10.043 
Fairburn, C. G. (2008). Cognitive behavior therapy and eating disorders (pp. xii, 324). Guilford Press.

Fairburn, C. G., \& Beglin, S. J. (1994). Assessment of eating disorders: Interview or self-report questionnaire? International Journal of Eating Disorders, 16(4), 363-370. $\quad$ https://doi.org/10.1002/1098-108X(199412)16:4<363::AID-

\section{$\underline{\text { EAT2260160405>3.0.CO;2-\# }}$}

Fairburn, C. G., Cooper, Z., \& O’Connor, M. (1993). The eating disorder examination. International Journal of Eating Disorders, 6, 1-8.

Fearon, P., Shmueli-Goetz, Y., Viding, E., Fonagy, P., \& Plomin, R. (2014). Genetic and environmental influences on adolescent attachment. Journal of Child Psychology and Psychiatry, 55(9), 1033-1041. https://doi.org/10.1111/jcpp.12171

Fearon, R. M. P., \& Roisman, G. I. (2017). Attachment theory: Progress and future directions. Current Opinion in Psychology, 15, 131-136. https://doi.org/10.1016/j.copsyc.2017.03.002

Fearon, R. P., Bakermans-Kranenburg, M. J., IJzendoorn, M. H. V., Lapsley, A.-M., \& Roisman, G. I. (2010). The Significance of Insecure Attachment and Disorganization in the Development of Children's Externalizing Behavior: A Meta-Analytic Study. Child Development, 81(2), 435-456. https://doi.org/10.1111/j.1467-8624.2009.01405.x

Feeney, J. A., Noller, P., \& Hanrahan, M. (1994). Assessing adult attachment. In M. B. Sperling \& W. H. Berman (Eds.). Attachment in adults: Clinical and developmental perspectives (pp. 128-152). Guilford Press. 
Fergusson, D. M., Woodward, L. J., \& Horwood, L. J. (2000). Risk factors and life processes associated with the onset of suicidal behaviour during adolescence and early adulthood. Psychological Medicine, 30, 23-39.

Fisher, C. A., Skocic, S., Rutherford, K. A., \& Hetrick, S. E. (2019). Family therapy approaches for anorexia nervosa. The Cochrane database of systematic reviews, 10(10),

CD004780.5.

\section{https://doi.org/10.1002/14651858.CD004780.pub4}

Flaskas, C. (2010). Frameworks for Practice in the Systemic Field: Part 1 Continuities and Transitions in Family Therapy Knowledge. Australian and New Zealand Journal of Family Therapy, 31(3), 232-247. https://doi.org/10.1375/anft.31.3.232

Folke, S., Daniel, S. I. F., Poulsen, S., \& Lunn, S. (2016). Client attachment security predicts alliance in a randomized controlled trial of two psychotherapies for bulimia nervosa. Psychotherapy Research, 26(4), 459-471. https://doi.org/10.1080/10503307.2015.1027319

Fonagy, P. \& Allison, E. (2014). The Role of Mentalizing and Epistemic Trust in the Therapeutic Relationship. Psychotherapy 51(3), 372-80.

Fonagy, P., \& Luyten, P. (2009). A developmental, mentalization-based approach to the understanding and treatment of borderline personality disorder. Development and Psychopathology, 21(4), 1355-1381. https://doi.org/10.1017/S0954579409990198

Fonagy, P., \& Target, M. (2006). The mentalization-focused approach to self pathology. Journal of Personality Disorders, 20(6), 544-576. https://doi.org/10.1521/pedi.2006.20.6.544 
Fonagy, P., Bateman, A. \& Luyten, P. (2012) Introduction and overview. In A. Bateman \& P. Fonagy (Eds.), Handbook of Mentalizing in Mental Health Practice (pp 3-42). Washington, DC: American Psychiatric Association Publishing.

Fonagy, P., Gergely, G., Jurist, E. L., \& Target, M. (2002). Affect Regulation, Mentalization and the Development of the Self. Other Press. https://doi.org/10.4324/9780429471643

Fonagy, P., Gergely, G., Jurist, E. L., Gergely, G., \& Jurist, E. L. (2002). Affect Regulation, Mentalization and the Development of the Self. Routledge. https://www.taylorfrancis.com/books/9780429471643

Fonagy, P., Leigh, T., Steele, M., Steele, H., Kennedy, R., Mattoon, G., Target, M., \& Gerber, A. (1996). The relation of attachment status, psychiatric classification, and response to psychotherapy. Journal of Consulting and Clinical Psychology, 64(1), 22-31. https://doi.org/10.1037//0022-006x.64.1.22

Fonagy, P., Luyten, P., \& Allison, E. (2015). Epistemic Petrification and the Restoration of Epistemic Trust: A New Conceptualization of Borderline Personality Disorder and Its Psychosocial Treatment. Journal of Personality Disorders, 29(5), 575-609. https://doi.org/10.1521/pedi.2015.29.5.575

Fonagy, P., Luyten, P., Allison, E., \& Campbell, C. (2017a). What we have changed our minds about: Part 1. Borderline personality disorder as a limitation of resilience. Borderline Personality Disorder and Emotion Dysregulation, 4(1), 11. https://doi.org/10.1186/s40479-017-0061-9

Fonagy, P., Luyten, P., Allison, E., \& Campbell, C. (2017b). What we have changed our minds about: Part 2. Borderline personality disorder, epistemic trust and the developmental significance of social communication. Borderline 
Personality Disorder and Emotion Dysregulation, 4(1), 9. https://doi.org/10.1186/s40479-017-0062-8

Fonagy, P., Luyten, P., Moulton-Perkins, A., Lee, Y.-W., Warren, F., Howard, S., Ghinai, R., Fearon, P., \& Lowyck, B. (2016). Development and Validation of a Self-Report Measure of Mentalizing: The Reflective Functioning Questionnaire. $\quad P L O S \quad$ ONE, $\quad$ 11(7), 0158678. https://doi.org/10.1371/journal.pone.0158678

Fonagy, P., Steele, M., Steele, H., Moran, G. S., \& Higgitt, A. C. (1991). The capacity for understanding mental states: The reflective self in parent and child and its significance for security of attachment. Infant Mental Health Journal, 12(3), 201-218.https://doi.org/10.1002/1097-0355(199123)12:3<201::AID-

\section{IMHJ2280120307>3.0.CO;2-7}

Fonagy, P., Target, M., Steele, H., \& Steele, M. (1998). Reflective-functioning manual, version 5.0, for application to adult attachment interviews. University College London, 161-2.

Forbush, K. T., Chen, P. Y., Hagan, K. E., Chapa, D. A., Gould, S. R., Eaton, N. R., \& Krueger, R. F. (2018). A new approach to eating-disorder classification: Using empirical methods to delineate diagnostic dimensions and inform care. International Journal of Eating Disorders, 51, 710-721. https://doi.org/10.1002/eat.22891

Forsberg, S., Darcy, A., Bryson, S. W., Arnow, K. D., Datta, N., Le Grange, D. and Lock, J. (2015). Psychological symptoms among parents of adolescents with anorexia nervosa: a descriptive examination of their presence and role in treatment outcome. Journal of Family Therapy. Available on Early View: doi: 10.1111/1467-6427.12088 
Forsberg, S., Lotempio, E., Bryson, S., Fitzpatrick, K.K., Le Grange, D., \& Lock, J. (2013). Therapeutic alliance in two treatments for adolescent anorexia nervosa. International Journal of Eating Disorders, 46, 34-38.

Forsberg, S., LoTempio, E., Bryson, S., Fitzpatrick, K.K., Le Grange, D. \& Lock, J. (2014) Parent-therapist alliance in family-based treatment for adolescents with anorexia nervosa. European Eating Disorders Review, 22: 53-58.

Fowkes, F. G., \& Fulton, P. M. (1991). Critical appraisal of published research: Introductory guidelines. BMJ : British Medical Journal, 302(6785), 11361140.

Fraley, R. C., \& Roisman, G. I. (2019). The development of adult attachment styles: Four lessons. Current Opinion in Psychology, 25, 26-30. https://doi.org/10.1016/j.copsyc.2018.02.008

Fraley, R. C., Waller, N. G., \& Brennan, K. A. (2000). An item response theory analysis of self-report measures of adult attachment. Journal of Personality and Social Psychology, 78(2), 350-365. https://doi.org/10.1037/0022$\underline{3514.78 .2 .350}$

Fraley, R.C. \& Roisman, G.I. (2014). III. Categories or dimensions? A taxometric analysis of the adult attachment interview. Monographs of the Society for Research in Child Development, 79, 36,50.

Fraley, R.C. \& Spieker, S. J. (2003). Are infant attachment patterns continuously or categorically distributed? A taxometric analysis of strange situation behavior. Developmental Psychology, 39, 387.

Frank, E. (1991). Conceptualization and Rationale for Consensus Definitions of Terms in Major Depressive Disorder: Remission, Recovery, Relapse, and 
Recurrence. Archives of General Psychiatry, 48(9), 851.

https://doi.org/10.1001/archpsyc.1991.01810330075011

Friedlander, M. L., Escudero, V., Horvath, A. O., Heatherington, L., Cabero, A., \& Martens, M. P. (2006). System for observing family therapy alliances: A tool for research and practice. Journal of Counseling Psychology, 53(2), 214-225. https://doi.org/10.1037/0022-0167.53.2.214

Frith, U., \& Frith, C. D. (2003). Development and neurophysiology of mentalizing. Philosophical Transactions of the Royal Society B: Biological Sciences, 358(1431), 459-473. https://doi.org/10.1098/rstb.2002.1218

Gabel, K., Pinhas, L., Eisler, I., Katzman, D., \& Heinmaa, M. The effect of multiple family therapy on weight gain in adolescents with anorexia nervosa: Pilot data. Journal of the Canadian Academy of Child and Adolescent Psychiatry, 23(3), p.196.

Garner, D. M. (2004). Eating Disorder Inventory-3. Professional manual. Psychological Assessment Resources.

Garner, D. M., Olmstead, M. P., \& Polivy, J. (1983). Development and validation of a multidimensional eating disorder inventory for anorexia nervosa and bulimia. International Journal of Eating Disorders, 2(2), 15-34. https://doi.org/10.1002/1098-108X(198321)2:2<15::AID-

\section{$\underline{\text { EAT2260020203>3.0.CO;2-6 }}$}

Garner, D. M., Olmsted, M. P., Bohr, Y., \& Garfinkel, P. E. (1982). The eating attitudes test: Psychometric features and clinical correlates. Psychological Medicine, 12(4), 871-878. https://doi.org/10.1017/s0033291700049163 
Geist, R., Heinmaa, M., Stephens, D., Davis, R. and Katzman, D. K. (2000) Comparison of family therapy and family group psychoeducation in adolescents with anorexia nervosa. Canadian Journal of Psychiatry, 45: 173.

George, C., Kaplan, N., \& Main, M. (1985). The Adult Attachment Interview. Unpublished protocol. Department of Psychology, University of California.

Godart, N., Berthoz, S., Curt, F., Perdereau, F., Rein, Z., Wallier, J. et al. (2012). A randomized controlled trial of adjunctive family therapy and treatment as usual following inpatient treatment for anorexia nervosa adolescents. PloS one, 7, e28249.

Goldberg, D., \& David, A. S. (1991). Family therapy and the glamour of science. Journal of Family Therapy, 13(1), 17-30. https://doi.org/10.1046/j..1991.00410.x

Goossens, L., Braet, C., Bosmans, G., \& Decaluwé, V. (2011). Loss of control over eating in pre-adolescent youth: The role of attachment and self-esteem. Eating Behaviors, 12(4), 289-295. https://doi.org/10.1016/j.eatbeh.2011.07.005

Goossens, L., Braet, C., Van Durme, K., Decaluwé, V., \& Bosmans, G. (2012). The parent-child relationship as predictor of eating pathology and weight gain in preadolescents. Journal of Clinical Child and Adolescent Psychology: The Official Journal for the Society of Clinical Child and Adolescent Psychology, American Psychological Association, Division 53, 41(4), 445-457. https://doi.org/10.1080/15374416.2012.660690

Goossens, L., Van Durme, K., Naeye, H., Verbeken, S., \& Bosmans, G. (2019). Insecure Attachment Moderates the Association between Thin Internalization and Girls' Eating Concerns. Journal of Child and Family Studies, 28(11), 3183-3192. https://doi.org/10.1007/s10826-019-01494-6 
Goossens, L., Van Durme, K., Van Beveren, M.-L., \& Claes, L. (2017). Do changes in affect moderate the association between attachment anxiety and body dissatisfaction in children? An experimental study by means of the Trier Social Stress Test. Eating Behaviors, 26, 83-88. https://doi.org/10.1016/j.eatbeh.2017.01.011

Granqvist, P. (2020). Attachment, culture, and gene-culture co-evolution: Expanding the evolutionary toolbox of attachment theory. Attachment \& Human Development, 1-24. https://doi.org/10.1080/14616734.2019.1709086

Gratz, K. L., \& Roemer, L. (2004). Multidimensional Assessment of Emotion Regulation and Dysregulation: Development, Factor Structure, and Initial Validation of the Difficulties in Emotion Regulation Scale. Journal of Psychopathology and Behavioral Assessment, 26, 41-54.

Graves, T. A., Tabri, N., Thompson-Brenner, H., Franko, D. L., Eddy, K. T., BourionBedes, S., Brown, A., Constantino, M. J., Flückiger, C., Forsberg, S., Isserlin, L., Couturier, J., Karlsson, G. P., Mander, J., Teufel, M., Mitchell, J. E., Crosby, R. D., Prestano, C., Satir, D. A., ... Thomas, J. J. (2017). A metaanalysis of the relation between therapeutic alliance and treatment outcome in eating disorders. International Journal of Eating Disorders, 50(4), 323-340. https://doi.org/10.1002/eat.22672

Green, J., Stanley, C., \& Peters, S. (2007). Disorganized attachment representation and atypical parenting in young school age children with externalizing disorder. Attachment \& Human Development 9(3), 207-222.

Green, J., Stanley, C., Smith, V., \& Goldwyn, R. (2000). A new method of evaluating attachment representations in young school-age children: The Manchester Child Attachment Story Task. Attachment \& Human Development, 2, 48-70. 
Groh, A. M., Roisman, G. I., van IJzendoorn, M. H., Bakermans-Kranenburg, M. J., \& Fearon, R. P. (2012). The significance of insecure and disorganized attachment for children's internalizing symptoms: A meta-analytic study. Child Development, 83, 591-610.

Ha, C., Sharp, C., Ensink, K., Fonagy, P., \& Cirino, P. (2013). The measurement of reflective function in adolescents with and without borderline traits. Journal of Adolescence, 36(6), 1215-1223.

https://doi.org/10.1016/j.adolescence.2013.09.008

Haley, J. (1973). Uncommon Therapy: The psychiatric techniques of MH Erickson. Norton.

Hallion, L. S., Steinman, S. A., Tolin, D. F., \& Diefenbach, G. J. (2018). Psychometric Properties of the Difficulties in Emotion Regulation Scale (DERS) and Its Short Forms in Adults With Emotional Disorders. Frontiers in Psychology, 9. https://doi.org/10.3389/fpsyg.2018.00539

Hamadi, L., \& Holliday, J. (2020). Moderators and mediators of outcome in treatments for anorexia nervosa and bulimia nervosa in adolescents: A systematic review of randomized controlled trials. International Journal of Eating Disorders, 53(1), 3-19. https://doi.org/10.1002/eat.23159

Hare-Mustin, R. T. (1987). The Problem of Gender in Family Therapy Theory. Family Process, 26(1), 15-27. https://doi.org/10.1111/j.1545-5300.1987.00015.x

Harlow, H. F., \& Harlow, M. (1966). Learning to Love. American Scientist, 54(3), $244-272$.

Harrison, A., Sullivan, S., Tchanturia, K., \& Treasure, J. (2009). Emotion recognition and regulation in anorexia nervosa. Clinical Psychology \& Psychotherapy, 16(4), 348-356. https://doi.org/10.1002/cpp.628 
Hawton, K., Saunders, K. E. A., \& O’Connor, R. C. (2012). Self-harm and suicide in adolescents. Lancet, 379(9834), 2373-2382. https://doi.org/10.1016/S0140$\underline{6736(12) 60322-5}$

Hayes, A. F. (2009). Beyond Baron and Kenny: Statistical Mediation Analysis in the New Millennium. Communication Monographs, 76(4), 408-420. https://doi.org/10.1080/03637750903310360

Hazan, C., \& Shaver, P. (1987). Romantic love conceptualized as an attachment process. Journal of Personality and Social Psychology, 52(3), 511-524. https://doi.org/10.1037/0022-3514.52.3.511

Herscovici, C. R., Kovalskys, I., \& Orellana, L. (2017). An Exploratory Evaluation of the Family Meal Intervention for Adolescent Anorexia Nervosa. Family Process, 56(2), 364-375. https://doi.org/10.1111/famp.12199

Hoffman, L. (1985). Beyond power and control: Toward a" second order" family systems therapy. Family Systems Medicine, 3(4), 381.

Hofmann, S. G., \& Hayes, S. C. (2019). The Future of Intervention Science: Process-Based Therapy. https://journals.sagepub.com/doi/full/10.1177/2167702618772296?casa_toke

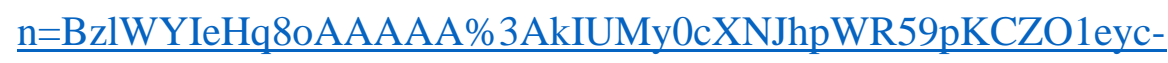
aqBIU8uDcfgMsXI9i9bFupV9cWOCTuNaQ2Z_HkPr5onPToNOU

Hofmann, S. G., Curtiss, J. E., \& Hayes, S. C. (2020). Beyond linear mediation: Toward a dynamic network approach to study treatment processes. Clinical Psychology Review, 76, 101824. https://doi.org/10.1016/j.cpr.2020.101824

Horvath, A., Del Re, A. C., Fluckiger, C. \& Symonds, D. (2011). Alliance in individual psychotherapy. Psychotherapy, 48, 9-16. 
House, J., Schmidt, U., Craig, M., Landau, S., Simic, M., Nicholls, D. et al. (2012). Comparison of specialist and non-specialist care pathways for adolescents with anorexia nervosa and related eating disorders. International Journal of Eating Disorders, 45, 949-956.

Hughes, E. K., Le Grange, D., Court, A., Yeo, M., Campbell, S., Whitelaw, M., et al. (2014). Implementation of family-based treatment for adolescents with anorexia nervosa. Journal of Pediatric Health Care, 28, 322-330.

Hughes, E. K., Sawyer, S. M., Accurso, E. C., Singh, S., \& Grange, D. L. (2019). Predictors of early response in conjoint and separated models of family-based treatment for adolescent anorexia nervosa. European Eating Disorders Review, 27(3), 283-294. https://doi.org/10.1002/erv.2668

Illing, V., Tasca, G. A., Balfour, L., \& Bissada, H. (2010). Attachment Insecurity Predicts Eating Disorder Symptoms and Treatment Outcomes in a Clinical Sample of Women. The Journal of Nervous and Mental Disease, 198(9), 653659. https://doi.org/10.1097/NMD.0b013e3181ef34b2

Inhelder, B., \& Piaget, J. (1958). The growth of logical thinking from childhood to adolescence. Basic Books.

Isserlin, L., \& Couturier, J. (2012). Therapeutic alliance and family-based treatment for adolescents with anorexia nervosa. Psychotherapy, 49(1), 46-51. https://doi.org/10.1037/a0023905

Jacobi, C., Hayward, C., de Zwaan, M., Kraemer, H. C., \& Agras, W. S. (2004). Coming to Terms With Risk Factors for Eating Disorders: Application of Risk Terminology and Suggestions for a General Taxonomy. Psychological Bulletin, 130(1), 19-65. https://doi.org/10.1037/0033-2909.130.1.19 
Jewell, T., Blessitt, E., Stewart, C., Simic, M., \& Eisler, I. (2016a). Family Therapy for Child and Adolescent Eating Disorders: A Critical Review. Family Process, 55(3), 577-594. https://doi.org/10.1111/famp.12242

Jewell, T., Collyer, H., Gardner, T., Tchanturia, K., Simic, M., Fonagy, P., \& Eisler, I. (2016b). Attachment and mentalization and their association with child and adolescent eating pathology: A systematic review. International Journal of Eating Disorders, 49(4), 354-373. https://doi.org/10.1002

Johnson, S. (2019). Attachment in action-Changing the face of 21st century couple therapy. Current Opinion in Psychology, 25, 101-104. https://doi.org/10.1016/j.copsyc.2018.03.007

Jones, J. D., Cassidy, J., \& Shaver, P. R. (2014). Parents' self-reported attachment styles: a review of links with parenting behaviors, emotions, and cognitions. Personality and Social Psychology Review, 19(1), 44-76.

Jones, J. D., Fraley, R. C., Ehrlich, K. B., Stern, J. A., Lejuez, C. W., Shaver, P. R., \& Cassidy, J. (2018). Stability of attachment style in adolescence: An empirical test of alternative developmental processes. Child development, 89, 871-880.

Juarascio, A., Manasse, S., Clark, K. E., Schaumberg, K., Kerrigan, S., Goldstein, S. P., ... \& Forman, E. (2020). Understanding the overlap and differences in terms describing patterns of maladaptive avoidance and intolerance of negative emotional states. Personality and Individual Differences, 158, 109859.

Katznelson, H., Falkenström, F., Daniel, S. I. F., Lunn, S., Folke, S., Pedersen, S. H., \& Poulsen, S. (2020). Reflective functioning, psychotherapeutic alliance, and outcome in two psychotherapies for bulimia nervosa. Psychotherapy, 57(2), 129-140. https://doi.org/10.1037/pst0000245 
Kazdin, A. E. (2007). Mediators and Mechanisms of Change in Psychotherapy Research. Annual Review of Clinical Psychology, 3(1), 1-27. https://doi.org/10.1146/annurev.clinpsy.3.022806.091432

Kazdin, A.E. and Weisz, J.R., 1998. Identifying and developing empirically supported child and adolescent treatments. Journal of Consulting and Clinical Psychology, 66(1), 19-36.

Keel, P. K., \& Brown, T. A. (2010). Update on course and outcome in eating disorders. International Journal of Eating Disorders, 43, 195-204. https://doi.org/10.1002/eat.20810

Kenny, T. E., Boyle, S. L., \& Lewis, S. P. (2020). \#recovery: Understanding recovery from the lens of recovery-focused blogs posted by individuals with lived experience. International Journal of Eating Disorders, 53(8), 1234-1243. https://doi.org/10.1002/eat.23221

Kerns, K. A. (2008). Attachment in middle childhood. In J. Cassidy, \& P. Shaver (Eds.), Handbook of Attachment: Theory, Research and Clinical implications (pp. 366-382). Guilford Press.

Kerns, K. A., \& Brumariu, L. E. (2016). Attachment in middle childhood. In J. Cassidy \& P.R. Shaver (Eds.), Handbook of attachment: Theory, research, and clinical applications (pp. 349-365). Guilford Press.

Kerns, K. A., Klepac, L., \& Cole, A. (1996). Peer Relationships and Preadolescents' Perceptions of Security in the Child-Mother Relationship. Developmental Psychology, 32(3), 457-466.

Kerns, K. A., Schlegelmilch, A., Morgan, T. A., \& Abraham, M. M. (2005). Assessing attachment in middle childhood. In K.A. Kerns \& R.A. Richardson (Eds.), Attachment in middle childhood (pp. 46-70). Guilford Press. 
Keski-Rahkonen, A., \& Tozzi, F. (2005). The process of recovery in eating disorder sufferers' own words: An Internet-based study. International Journal of Eating Disorders, 37, S80-S86. https://doi.org/10.1002/eat.20123

Keys, A., Brozek, J., Henschel, A., Mickelson, O., \& Taylor, H.L. (1950). The biology of human starvation. University of Minnesota Press.

Khalsa, S. S., Portnoff, L. C., McCurdy-McKinnon, D., \& Feusner, J. D. (2017). What happens after treatment? A systematic review of relapse, remission, and recovery in anorexia nervosa. Journal of Eating Disorders, 5(1), 20. https://doi.org/10.1186/s40337-017-0145-3

Kirschbaum, C., Pirke, K.-M., \& Hellhammer, D. H. (1993). The 'Trier Social Stress Test' - A Tool for Investigating Psychobiological Stress Responses in a Laboratory Setting. Neuropsychobiology, 28(1-2), 76-81. https://doi.org/10.1159/000119004

Kobak, R. R., Cole, H. E., Ferenz-Gillies, R., Fleming, W. S., \& Gamble, W. (1993). Attachment and emotion regulation during mother-teen problem solving: A control theory analysis. Child Development, 64(1), 231-245.

Kog, E., \& Vandereycken, W. (1989). The speculations: An overview of theories about eating disorder families. The Family Approach to Eating Disorders, 724.

Kordy, H., Krämer, B., Palmer, R. L., Papezova, H., Pellet, J., Richard, M., Treasure, J., \& B6, C. A. (2002). Remission, recovery, relapse, and recurrence in eating disorders: Conceptualization and illustration of a validation strategy. Journal of Clinical Psychology, 58(7), 833-846. https://doi.org/10.1002/jclp.2013 
Kosmerly, S., Waller, G. \& Robinson, A. L. (2015) Clinician adherence to guidelines in the delivery of family-based therapy for eating disorders. International Journal of Eating Disorders, 48: 223-229.

Kraemer, H. C., Kazdin, A. E., Offord, D. R., Kessler, R. C., Jensen, P. S., \& Kupfer, D. J. (1997). Coming to terms with the terms of risk. Archives of General Psychiatry, 54(4), 337-343. https://doi.org/10.1001/archpsyc.1997.01830160065009

Kraemer, H. C., Wilson, G. T., Fairburn, C. G., \& Agras, W. S. (2002). Mediators and Moderators of Treatment Effects in Randomized Clinical Trials. Archives of General Psychiatry, 59(10), 877. https://doi.org/10.1001/archpsyc.59.10.877

Kuipers, G. S., \& Bekker, M. H. J. (2012). Attachment, mentalization and eating disorders: A review of studies using the Adult Attachment Interview. Current Psychiatry Reviews, 8(4), 326-336.

\section{https://doi.org/10.2174/157340012803520478}

Kuipers, G. S., Hollander, S. den, van der Ark, L. A., \& Bekker, M. H. J. (2017). Recovery from eating disorder 1 year after start of treatment is related to better mentalization and strong reduction of sensitivity to others. Eating and Weight Disorders - Studies on Anorexia, Bulimia and Obesity, 22(3), 535-547. https://doi.org/10.1007/s40519-017-0405-X

Kuipers, G. S., Loenhout, Z. van, Ark, L. A. van der, \& Bekker, M. H. J. (2018). Is reduction of symptoms in eating disorder patients after 1 year of treatment related to attachment security and mentalization? Eating Disorders, 26(3), 263-269. https://doi.org/10.1080/10640266.2017.1384916

Landrum, A.R., Eaves, B.S. \& Shafto, P. (2015). Learning to trust and trusting to learn: A theoretical framework. Trends in Cognitive Sciences, 19, 3, 109-111. 
Lang, K., Dapelo, M. M., Khondoker, M., Morris, R., Surguladze, S., Treasure, J., \& Tchanturia, K. (2015). Exploring emotion recognition in adults and adolescents with anorexia nervosa using a body motion paradigm. European Eating Disorders Review: The Journal of the Eating Disorders Association, 23(4), 262-268. https://doi.org/10.1002/erv.2358

Lask, B. (2000). Overview of Management. In B. Lask and R. Bryant-Waugh (Eds.), Anorexia nervosa and related eating disorders in childhood and adolescence (pp.167-185). Taylor \& Francis.

Le Grange, D., Accurso, E., Lock, J., Agras, W. S., \& Bryson, S. W. (2014). Early weight gain predicts outcome in two treatments for adolescent anorexia nervosa. International Journal of Eating Disorders, 47, 124-129.

Le Grange, D., Crosby, R. D., \& Lock, J. (2008). Predictors and moderators of outcome in family-based treatment for adolescent bulimia nervosa. Journal of the American Academy of Child and Adolescent Psychiatry, 47, 464-470.

Le Grange, D., Crosby, R. D., Rathouz, P. J., \& Leventhal, B. L. (2007). A randomised controlled comparison of family-based treatment and supportive psychotherapy for adolescent bulimia nervosa. Archives of General Psychiatry, 64, 1049-1056.

Le Grange, D., Eckhardt, S., Grave, R. D., Crosby, R. D., Peterson, C. B., Keery, H., Lesser, J., \& Martell, C. (2020). Enhanced cognitive-behavior therapy and family-based treatment for adolescents with an eating disorder: A nonrandomized effectiveness trial. Psychological Medicine, 1-11. https://doi.org/10.1017/S0033291720004407 
Le Grange, D., Eisler, I., Dare, C., \& Hodes, M. (1992). Family criticism and selfstarvation: A study of expressed emotion. Journal of Family Therapy, 14, 177192.

Le Grange, D., Eisler, I., Dare, C., \& Russell, G. F. (1992). Evaluation of family treatments in adolescent anorexia nervosa: A pilot study. International Journal of Eating Disorders, 12(4), 347-357. https://doi.org/10.1002/1098108X\%28199212\%2912:4\%3C347::AID-EAT2260120402\%3E3.0.CO;2-W

Le Grange, D., Hughes, E. K., Court, A., Yeo, M., Crosby, R. D., \& Sawyer, S. M. (2016). Randomized Clinical Trial of Parent-Focused Treatment and FamilyBased Treatment for Adolescent Anorexia Nervosa. Journal of the American Academy of Child \& Adolescent Psychiatry, 55(8), 683-692. https://doi.org/10.1016/j.jaac.2016.05.007

Le Grange, D., Huryk, K. M., Murray, S. B., Hughes, E. K., Sawyer, S. M., \& Loeb, K. L. (2019). Variability in remission in family therapy for anorexia nervosa. International Journal of Eating Disorders, 52(9), 996-1003. https://doi.org/10.1002/eat.23138

Le Grange, D., \& Lock, J. (2007). Treating Bulimia in Adolescents: A Family-Based Approach (1st edition). Guilford Press.

Le Grange, D., Lock, J., Agras, W. S., Bryson, S. W., \& Jo, B. (2015). Randomized Clinical Trial of Family-Based Treatment and Cognitive-Behavioral Therapy for Adolescent Bulimia Nervosa. Journal of the American Academy of Child \& Adolescent Psychiatry, $54(11)$ 886-894. https://doi.org/10.1016/j.jaac.2015.08.008 
Le Grange, D., Lock, J., Loeb, K., \& Nicholls, D. (2010). Academy for Eating Disorders position paper: The role of the family in eating disorders. International Journal of Eating Disorders, 43(1), 1-5.

Le Grange, D., O’Connor, M., Hughes, E. K., Macdonald, J., Little, K., \& Olsson, C. A. (2014). Developmental antecedents of abnormal eating attitudes and behaviors in adolescence. International Journal of Eating Disorders, 47(7), 813-824. https://doi.org/10.1002/eat.22331

Leff, J. \& Vaughn, C. (1985). Expressed Emotion in Families. Guilford Press.

Leslie, M., Halls, D., Leppanen, J., Sedgewick, F., Smith, K., Hayward, H., Lang, K., Fonville, L., Simic, M., Mandy, W., Nicholls, D., Murphy, D., Williams, S., \& Tchanturia, K. (2020). Neural Correlates of Theory of Mind Are Preserved in Young Women With Anorexia Nervosa. Frontiers in Psychology, 11. https://doi.org/10.3389/fpsyg.2020.568073

Levy, K. N., Kivity, Y., Johnson, B. N., \& Gooch, C. V. (2018). Adult attachment as a predictor and moderator of psychotherapy outcome: A meta-analysis. Journal of Clinical Psychology, 74(11), 1996-2013. https://doi.org/10.1002/jclp.22685

Lock, J. (2015). An update on evidence-based psychosocial treatments for eating disorders in children and adolescents. Journal of Clinical Child and Adolescent Psychology. 44:707-721.

Lock, J., Agras, W. S., Bryson, S., \& Kraemer, H. C. (2005). A Comparison of Shortand Long-Term Family Therapy for Adolescent Anorexia Nervosa. Journal of the American Academy of Child \& Adolescent Psychiatry, 44(7), 632-639. https://doi.org/10.1097/01.chi.0000161647.82775.0a 
Lock, J., Couturier, J., Bryson, S., \& Agras, W. S. (2006). Predictors of dropout and remission in family therapy for adolescent anorexia nervosa in a randomized clinical trial. International Journal of Eating Disorders, 39, 639-647.

Lock, J., Le Grange, Daniel, Agras, W. S., \& Dare, C. (2001). Treatment Manual for Anorexia Nervosa: A Family-Based Approach (First Edition). The Guilford Press.

Lock, J., Le Grange, D., Agras, W. S., Moye, A., Bryson, S. W., \& Jo, B. (2010). Randomized Clinical Trial Comparing Family-Based Treatment With Adolescent-Focused Individual Therapy for Adolescents With Anorexia Nervosa. Archives of General Psychiatry, 67(10), 1025-1032. https://doi.org/10.1001/archgenpsychiatry.2010.128

Lock, J., Le Grange, Daniel, Agras, W. S., \& Dare, C. (2001). Treatment Manual for Anorexia Nervosa: A Family-Based Approach (First Edition). The Guilford Press.

Loeb, K. L., Walsh, B. T., Lock, J., Le Grange, D., Jones, J., Marcus, S., et al. (2007). Open trial of family-based treatment for full and partial anorexia nervosa in adolescence: Evidence of successful dissemination. Journal of the American Academy of Child \& Adolescent Psychiatry, 46, 792-800.

Long, M., Verbeke, W., Ein-Dor, T., \& Vrtička, P. (2020). A Functional NeuroAnatomical Model of Human Attachment (NAMA): Insights from First- and Second-Person Social Neuroscience. https://doi.org/10.1016/j.cortex.2020.01.010

Lulé, D., Schulze, U. M. E., Bauer, K., Schöll, F., Müller, S., Fladung, A.-K., \& Uttner, I. (2014). Anorexia nervosa and its relation to depression, anxiety, alexithymia 
and emotional processing deficits. Eating and Weight Disorders: EWD, 19(2), 209-216. https://doi.org/10.1007/s40519-014-0101-z

Luyten, P., Campbell, C., Allison, E., \& Fonagy, P. (2020). The Mentalizing Approach to Psychopathology: State of the Art and Future Directions. Annual Review of Clinical Psychology, 16. Advance online publication: https://doi.org/10.1146/annurev-clinpsy-071919-015355

Luyten, P., Malcorps, S., Fonagy, P., \& Ensink, K. (2019). Assessment of mentalizing. In A. Bateman \& P. Fonagy (Eds.), Handbook of Mentalizing in Mental Health Practice (pp 37-62). Washington, DC: American Psychiatric Association Publishing.

Madden, S., Miskovic-Wheatley, J., Wallis, A., Kohn, M., Lock, J., Grange, D. L., Jo, B., Clarke, S., Rhodes, P., Hay, P., \& Touyz, S. (2015). A randomized controlled trial of in-patient treatment for anorexia nervosa in medically unstable adolescents. Psychological Medicine, 45(2), 415-427. https://doi.org/10.1017/S0033291714001573

Main, M., \& Solomon, J. (1986). Discovery of an insecure-disorganized/disoriented attachment pattern. In T.B. Brazelton \& M.W. Yogman (Eds.), Affective Development in Infancy (pp. 95-124). Ablex Publishing.

Maloney, M. J., McGuire, J. B., \& Daniels, S. R. (1988). The Children's Eating Attitudes Test (ChEAT). Journal of the American Academy of Child and Adolescent Psychiatry, 44, 632-639.

Marzola, E., Knatz, S., Murray, S. B., Rockwell, R., Boutelle, K., Eisler, I., et al. (2015). Short-term intensive family therapy for adolescent eating disorders: 30-month outcome. European Eating Disorders Review, 23(3), 210-218. 
Matsumoto, D., \& Ekman, P. (1988). Japanese and Caucasian facial expressions of emotion (JACFEE) and neutral faces (JACNeuF). Intercultual and Emotion Research Laboratory, Department of Psychology, San Francisco State University. https://ci.nii.ac.jp/naid/10011195722/

Mayer, B., Muris, P., Meesters, C., \& Zimmermann-van Beuningen, R. (2009). Brief report: Direct and indirect relations of risk factors with eating behavior problems in late adolescent females. Journal of Adolescence, 32(3), 741-745. https://doi.org/10.1016/j.adolescence.2008.12.002

McAdams, C. J., \& Krawczyk, D. C. (2011). Impaired neural processing of social attribution in anorexia nervosa. Psychiatry Research, 194(1), 54-63. https://doi.org/10.1016/j.pscychresns.2011.06.016

McCaig, D., Elliott, M. T., Siew, C. S. Q., Walasek, L., \& Meyer, C. (2019). Profiling commenters on mental health-related online forums: A methodological example focusing on eating disorder-related commenters. JMIR Mental Health, 6, e12555. https://doi.org/10.2196/12555

Meesters, C., Muris, P., Hoefnagels, C., \& van Gemert, M. (2007). Social and family correlates of eating problems and muscle preoccupation in young adolescents. Eating Behaviors, 8(1), 83-90. https://doi.org/10.1016/j.eatbeh.2006.02.002

Milan, S., \& Acker, J. C. (2014). Early attachment quality moderates eating disorder risk among adolescent girls. Psychology \& Health, 29(8), 896-914. https://doi.org/10.1080/08870446.2014.896463

Miles, S., Gnatt, I., Phillipou, A., \& Nedeljkovic, M. (2020). Cognitive flexibility in acute anorexia nervosa and after recovery: A systematic review. Clinical Psychology Review, 81, 101905. https://doi.org/10.1016/j.cpr.2020.101905

Minuchin, S. (1974). Families \& family therapy (pp. viii, 268). Harvard U. Press. 
Minuchin, S., Baker, L., Rosman, B. L., Liebman, R., Milman, L., \& Todd, T. C. (1975). A conceptual model of psychosomatic illness in children: Family organization and family therapy. Archives of General Psychiatry, 32(8), 10311038. https://doi.org/10.1001/archpsyc.1975.01760260095008

Minuchin, S., Rosman, B. L., Baker, L., \& Minuchin, S. (1978). Psychosomatic Families: Anorexia Nervosa in Context. Harvard University Press.

Moessner, M., Feldhege, J., Wolf, M., \& Bauer, S. (2018). Analyzing big data in social media: Text and network analyses of an eating disorder forum. International Journal of Eating Disorders, 51, 656-667. https://doi.org/10.1002/eat.22878

Moher, D., Liberati, A., Tetzlaff, J., Altman, D. G., \& PRISMA Group. (2009). Preferred reporting items for systematic reviews and meta-analyses: The PRISMA statement. PLoS Medicine, 6(7), e1000097. https://doi.org/10.1371/journal.pmed.1000097

Morgan, H. G., \& Russell, G. F. M. (1975). Value of family background and clinical features as predictors of long-term outcome in anorexia nervosa: Four-year follow-up study of 41 patients. Psychological Medicine, 5(4), 355-371. https://doi.org/10.1017/S0033291700056981

Morris, R., Bramham, J., Smith, E., \& Tchanturia, K. (2014). Empathy and social functioning in anorexia nervosa before and after recovery. Cognitive Neuropsychiatry, 19(1), $47-57$.

\section{https://doi.org/10.1080/13546805.2013.794723}

Murray, H. A. (1943). Thematic apperception test manual. Harvard University Press.

Murray, S. B., Griffiths, S. \& Le Grange, D. (2014), The role of collegial alliance in family-based treatment of adolescent anorexia nervosa: A pilot study. International Journal of Eating Disorders, 47: 418-421. 
Murray, S. B., Quintana, D. S., Loeb, K. L., Griffiths, S., \& Grange, D. L. (2019). Treatment outcomes for anorexia nervosa: A systematic review and metaanalysis of randomized controlled trials. Psychological Medicine, 49(4), 535544. https://doi.org/10.1017/S0033291718002088

National Institute for Clinical Excellence (2004). Eating disorders: Core interventions in the treatment and management of anorexia nervosa, bulimia nervosa and related eating disorders. NICE.

National Institute for Health and Care Excellence. (2015). Children's attachment: Attachment in children and young people who are adopted from care, in care or at high risk of going into care (NG26). NICE. https://www.nice.org.uk/guidance/ng26

National Institute for Health and Care Excellence. (2017). Eating disorders: Recognition and treatment. NG69. NICE. https://www.nice.org.uk/guidance/ng69

Nowicki, S., \& Duke, M. P. (1994). Individual differences in the nonverbal communication of affect: The diagnostic analysis of nonverbal accuracy scale. Journal of Nonverbal Behavior, 18(1), 9-35. https://doi.org/10.1007/BF02169077

Nyman-Carlsson, E., Birgegård, A., Engström, I., Gustafsson, S. A., \& Nevonen, L. (2019). Predictors of outcome among young adult patients with anorexia nervosa in a randomised controlled trial. European Eating Disorders Review, 27(1), 76-85. https://doi.org/10.1002/erv.2630

Nyman-Carlsson, E., Norring, C., Engström, I., Gustafsson, S. A., Lindberg, K., Paulson-Karlsson, G., \& Nevonen, L. (2020). Individual cognitive behavioral therapy and combined family/individual therapy for young adults with 
Anorexia nervosa: A randomized controlled trial. Psychotherapy Research, 30(8), 1011-1025. https://doi.org/10.1080/10503307.2019.1686190

O'Kearney, R. (1996). Attachment disruption in anorexia nervosa and bulimia nervosa: A review of theory and empirical research. The International Journal of Eating Disorders, 20(2), 115-127. https://doi.org/10.1002/(SICI)1098$\underline{108 X(199609) 20: 2<115:: A I D-E A T 1>3.0 . C O ; 2-J}$

Oldershaw, A., Hambrook, D., Stahl, D., Tchanturia, K., Treasure, J., \& Schmidt, U. (2011). The socio-emotional processing stream in Anorexia Nervosa. Neuroscience and Biobehavioral Reviews, 35(3), 970-988. https://doi.org/10.1016/j.neubiorev.2010.11.001

Oldershaw, A., Hambrook, D., Tchanturia, K., Treasure, J., \& Schmidt, U. (2010). Emotional theory of mind and emotional awareness in recovered anorexia nervosa patients. Psychosomatic Medicine, 72(1), 73-79. https://doi.org/10.1097/PSY.0b013e3181c6c7ca

Orzolek-Kronner, C. (2002). The Effect of Attachment Theory in the Development of Eating Disorders: Can Symptoms Be Proximity-Seeking? Child and Adolescent Social Work Journal, 19(6), 421-435. https://doi.org/10.1023/A:1021141612634

Pace, C. S., Guiducci, V., \& Cavanna, D. (2016). A controlled study of attachment representations and emotion regulation in female adolescents with anorexia nervosa. Mediterranean Journal of Clinical Psychology, 4(1), Article 1. https://doi.org/10.6092/2282-1619/2016.4.1187

Pace, U., Cacioppo, M., \& Schimmenti, A. (2012). The moderating role of father's care on the onset of binge eating symptoms among female late adolescents 
with insecure attachment. Child Psychiatry and Human Development, 43(2), 282-292. https://doi.org/10.1007/s10578-011-0269-7

Pedersen, S. H., Lunn, S., Katznelson, H., \& Poulsen, S. (2012). Reflective functioning in 70 patients suffering from bulimia nervosa. European Eating Disorders Review: The Journal of the Eating Disorders Association, 20(4), 303-310. https://doi.org/10.1002/erv.2158

Pennesi, J.-L., \& Wade, T. D. (2016). A systematic review of the existing models of disordered eating: Do they inform the development of effective interventions? Clinical Psychology Review, 43, 175-192. https://doi.org/10.1016/j.cpr.2015.12.004

Pereira, T., Lock, J., \& Oggins, J. (2006). Role of therapeutic alliance in family therapy for adolescent anorexia nervosa. International Journal of Eating Disorders. $39,677-84$.

Petkova, H., Simic, M., Nicholls, D., Ford, T., Prina, A. M., Stuart, R., Livingstone, N., Kelly, G., Macdonald, G., Eisler, I., Gowers, S., Barrett, B. M., \& Byford, S. (2019). Incidence of anorexia nervosa in young people in the UK and Ireland: A national surveillance study. BMJ Open, 9(10), e027339. https://doi.org/10.1136/bmjopen-2018-027339

Pike, K. M. (2005). Assessment of anorexia nervosa. International Journal of Eating Disorders, 37(S1), S22-S25. https://doi.org/10.1002/eat.20111

Pinquart, M., Feußner, C., \& Ahnert, L. (2013). Meta-analytic evidence for stability in attachments from infancy to early adulthood. Attachment \& Human Development, $15(2)$, $189-218$. https://doi.org/10.1080/14616734.2013.746257 
Polivy, J., \& Herman, C. P. (2002). Causes of eating disorders. Annual Review of Psychology, 53 , $187-213$. https://doi.org/10.1146/annurev.psych.53.100901.135103

Pote, H., Stratton, P., Cottrell, D., Boston, P. \& Shapiro, D. (2001) Systemic Family Therapy Manual. University of Leeds, Family Therapy Research Centre.

Prochaska, J. O., \& DiClemente, C. C. (1983). Stages and processes of self-change of smoking: toward an integrative model of change. Journal of Consulting and Clinical Psychology, 51, 390-395. $\quad$ https://doi.org/10.1037/0022$\underline{006 X .51 .3 .390}$

Pugh, M., \& Waller, G. (2017). Understanding the 'Anorexic Voice' in Anorexia Nervosa. Clinical Psychology \& Psychotherapy, 24(3), 670-676. https://doi.org/10.1002/cpp.2034

Python Software Foundation (2017). Python Language Reference. 3.6.3.ed. Retrieved from http://www.python.org.

Rienecke, R.D., Accurso, E.C., Lock, J. \& Le Grange, D. (2016). Expressed emotion, family functioning, and treatment outcome for adolescents with anorexia nervosa. European Eating Disorders Review, 24(1), pp.43-51.

Ringer, F., \& Crittenden, P. M. (2007). Eating disorders and attachment: The effects of hidden family processes on eating disorders. European Eating Disorders Review: The Journal of the Eating Disorders Association, 15(2), 119-130. https://doi.org/10.1002/erv.761

Robin, A. L., Siegel, P. T., Moye, A. W., Gilroy, M., Dennis, A. B., \& Sikand, A. (1999). A controlled comparison of family versus individual therapy for adolescents with anorexia nervosa. Journal of the American Academy of Child 
and Adolescent Psychiatry, 38(12), 1482-1489. https://doi.org/10.1097/00004583-199912000-00008

Robinson, A. \& Kosmerly, S. (2015) The Influence of Clinician Emotion on Decisions in Child and Adolescent Eating Disorder Treatment: A Survey of Self and Others. Eating Disorders, 23:2, 163-176.

Robinson, A.L., Dolhanty, J. \& Greenberg, L. (2015). Emotion-Focused Family Therapy for Eating Disorders in Children and Adolescents. Clinical Psychology \& Psychotherapy, 22(1), pp.75-82.

Robinson, A.L., Strahan, E., Girz, L., Wilson, A., \& Boachie, A. (2013). 'I know I can help you': parental self-efficacy predicts adolescent outcomes in family-based therapy for eating disorders. European Eating Disorder Review, 21, 108-114.

Robinson, P., Sklaarderud, F., \& Sommerfeldt, B. (2018). Hunger: Mentalizationbased treatments for eating disorders. Springer.

Rodgers, R. F., Berry, R., \& Franko, D. L. (2018). Eating Disorders in Ethnic Minorities: An Update. Current Psychiatry Reports, 20(10), 90. https://doi.org/10.1007/s11920-018-0938-3

Roisman, G. I., Holland, A., Fortuna, K., Fraley, R. C., Clausell, E., \& Clarke, A. (2007). The Adult Attachment Interview and self-reports of attachment style: An empirical rapprochement. Journal of Personality and Social Psychology, 92(4), 678-697. https://doi.org/10.1037/0022-3514.92.4.678

Rossouw, T. I., \& Fonagy, P. (2012). Mentalization-based treatment for self-harm in adolescents: A randomized controlled trial. Journal of the American Academy of Child and Adolescent Psychiatry, 51(12), 1304-1313.e3. https://doi.org/10.1016/j.jaac.2012.09.018 
Rothschild-Yakar, L., Levy-Shiff, R., Fridman-Balaban, R., Gur, E., \& Stein, D. (2010). Mentalization and Relationships With Parents as Predictors of Eating Disordered Behavior. The Journal of Nervous and Mental Disease, 198(7), 501-507. https://doi.org/10.1097/NMD.0b013e3181e526c8

Rothschild-Yakar, L., Stein, D., Goshen, D., Shoval, G., Yacobi, A., Eger, G., Kartin, B., \& Gur, E. (2019). Mentalizing Self and Other and Affect Regulation Patterns in Anorexia and Depression. Frontiers in Psychology, 10. https://doi.org/10.3389/fpsyg.2019.02223

Rothschild-Yakar, L., Waniel, A., \& Stein, D. (2013). Mentalizing in Self vs. Parent Representations and Working Models of Parents as Risk and Protective Factors From Distress and Eating Disorders. The Journal of Nervous and Mental Disease, 201(6), 510-518. https://doi.org/10.1097/NMD.0b013e3182948316 Royston, P., \& White, I. R. (2011). Multiple Imputation by Chained Equations (MICE): Implementation in Stata. Journal of Statistical Software, 45(1), 1-20. https://doi.org/10.18637/jss.v045.i04

Russell, G. F., Szmukler, G. I., Dare, C., \& Eisler, I. (1987). An evaluation of family therapy in anorexia nervosa and bulimia nervosa. Archives of General Psychiatry, 44(12), 1047-1056.

https://doi.org/10.1001/archpsyc.1987.01800240021004

Russell, T. A., Schmidt, U., Doherty, L., Young, V., \& Tchanturia, K. (2009). Aspects of social cognition in anorexia nervosa: Affective and cognitive theory of mind. Psychiatry $\quad$ Research, 168(3), 181-185. https://doi.org/10.1016/j.psychres.2008.10.028

Sadeh-Sharvit, S., Arnow, K. D., Osipov, L., Lock, J. D., Jo, B., Pajarito, S., Brandt, H., Dodge, E., Halmi, K. A., Johnson, C., Kaye, W., Wilfley, D., \& Agras, W. 
S. (2018). Are parental self-efficacy and family flexibility mediators of treatment for anorexia nervosa? International Journal of Eating Disorders, 51(3), 275-280. https://doi.org/10.1002/eat.22826

Salaminiou, E., Campbell, M., Simic, M., Kuipers, E., \& Eisler, I. (2015) Intensive multi family therapy for adolescent anorexia nervosa: An open study of 30 families. Journal of Family Therapy. doi:10.1111/14676427.12075

Salbach-Andrae, H., Lenz, K., Simmendinger, N., Klinkowski, N., Lehmkuhl, U., \& Pfeiffer, E. (2008). Psychiatric Comorbidities among Female Adolescents with Anorexia Nervosa. Child Psychiatry and Human Development, 39(3), 261272. https://doi.org/10.1007/s10578-007-0086-1

Saure, E., Laasonen, M., Lepistö-Paisley, T., Mikkola, K., Ålgars, M., \& Raevuori, A. (2020). Characteristics of autism spectrum disorders are associated with longer duration of anorexia nervosa: A systematic review and meta-analysis. International Journal of Eating Disorders, 53(7), 1056-1079. https://doi.org/10.1002/eat.23259

Schaumberg, K., Zerwas, S. C., Bulik, C. M., Fiorentini, C., \& Micali, N. (2020). Prospective associations between childhood social communication processes and adolescent eating disorder symptoms in an epidemiological sample. European Child \& Adolescent Psychiatry. https://doi.org/10.1007/s00787-

\section{$\underline{020-01655-9}$}

Schmidt, U., \& Asen, E. (2005). Editorial: Does multi-family day treatment hit the spot that other treatments cannot reach?. Journal of Family Therapy, 27, 101103 https://doi.org/10.1111/j.1467-6427.2005.00302.x

Schmidt, U., Lee, S., Beecham, J., Perkins, S., Treasure, J., Yi, I., Winn, S., Robinson, P., Murphy, R., Keville, S., Johnson-Sabine, E., Jenkins, M., Frost, S., Dodge, 
L., Berelowitz, M., \& Eisler, I. (2007). A randomized controlled trial of family therapy and cognitive behavior therapy guided self-care for adolescents with bulimia nervosa and related disorders. The American Journal of Psychiatry, 164(4), 591-598. https://doi.org/10.1176/appi.ajp.164.4.591

Schmidt, U., Lee, S., Beecham, J., Perkins, S., Treasure, J., Yi, I., Eisler, I. (2007). A randomized controlled trial of family therapy and cognitive behaviour therapy guided self-care for adolescents with bulimia nervosa and related conditions. American Journal of Psychiatry, 164, 591-598.

Schmidt, U., \& Treasure, J. (2006). Anorexia nervosa: Valued and visible. A cognitive-interpersonal maintenance model and its implications for research and practice. British Journal of Clinical Psychology, 45(3), 343-366. https://doi.org/10.1348/014466505X53902

Schulte-Rüther, M., Mainz, V., Fink, G. R., Herpertz-Dahlmann, B., \& Konrad, K. (2012). Theory of Mind and the Brain in Anorexia Nervosa: Relation to Treatment Outcome. Journal of the American Academy of Child \& Adolescent Psychiatry, 51(8), 832-841.e11. https://doi.org/10.1016/j.jaac.2012.06.007

Scott, S., Briskman, J., Woolgar, M., Humayun, S., \& O’Connor, T. G. (2011). Attachment in adolescence: Overlap with parenting and unique prediction of behavioural adjustment. Journal of Child Psychology and Psychiatry, and Allied Disciplines, 52(10), 1052-1062. https://doi.org/10.1111/j.1469$\underline{7610.2011 .02453 . x}$

Selvini Palazzoli, M. (1974). Self-Starvation: From the Intrapsychic to the Transpersonal Approach to Anorexia Nervosa. Chaucer. https://www.bookdepository.com/Self-Starvation-Mara-Selvini-

\section{$\underline{\text { Palazzoli/9781568218229 }}$}


Sharp, C., Barr, C., \& Vanwoerden, S. (2018). Hypermentalizing: The development and validation of a self-report measure. Unpublished Manuscript.

Sharp, C., Pane, H., Ha, C., Venta, A., Patel, A. B., Sturek, J., \& Fonagy, P. (2011). Theory of Mind and Emotion Regulation Difficulties in Adolescents With Borderline Traits. Journal of the American Academy of Child \& Adolescent Psychiatry, 50(6), 563-573.e1. https://doi.org/10.1016/j.jaac.2011.01.017

Sharpe, T. M., Killen, J. D., Bryson, S. W., Shisslak, C. M., Estes, L. S., Gray, N., Crago, M., \& Taylor, C. B. (1998). Attachment style and weight concerns in preadolescent and adolescent girls. The International Journal of Eating Disorders, $\quad$ 23(1), 39-44. $\quad$ https://doi.org/10.1002/(sici)1098$\underline{108 x(199801) 23: 1<39:: \text { aid-eat5>3.0.co;2-2 }}$

Shmueli-Goetz, Y., Target, M., Fonagy, P., \& Datta, A. (2008). The Child Attachment Interview: A psychometric study of reliability and discriminant validity. Developmental Psychology, 44(4), 939-956. https://doi.org/10.1037/0012-

\section{$\underline{1649.44 .4 .939}$}

Simic, M., \& Eisler, I. (2015). Multi family therapy. In K. Loeb, D. Le Grange \& J. Lock (Eds). Family therapy for adolescent eating and weight disorders (pp. 110-138). Routledge.

Simic, M., Anderson, L.K., Berner, L., Peck, S.K., Hunt, K., Kaye, W. \& Eisler, I. (In Press). When Family Therapy isn't Enough: New Treatment Directions for Highly Anxious and Dysregulated Adolescents with Anorexia Nervosa. In S.B. Murray, L.K. Anderson \& L. Cohn (Eds). Innovations in Family Therapy for Eating Disorders: Novel Treatment Developments, Patient Insights, and the Role of Carers. Routledge. 
Simic, M., Jewell, T., \& Eisler, I. (2020). Beneath the Surface of Expressed Emotion (EE): The Clinical Relevance of Possible Mechanisms Underlying EE in Eating Disorders. In RD Rienecke \& D Le Grange (Eds.). Eating Disorders and Expressed Emotion (pp. 33-57). Routledge.

Simonsen, C. B., Jakobsen, A. G., Grøntved, S., \& Telléus, G. K. (2020). The mentalization profile in patients with eating disorders: A systematic review and meta-analysis. Nordic Journal of Psychiatry, 74(5), 311-322. https://doi.org/10.1080/08039488.2019.1707869

Simpson, A., Hannigan, B., Coffey, M., Jones, A., Barlow, S., Cohen,R.,... \& Faulkner, A. (2016). Cross-national comparative mixed-methods case study of recovery-focused mental health care planning and co-ordination: Collaborative Care Planning Project (COCAPP). NIHR Journals Library; 2016 Feb. (Health Services and Delivery Research, No. 4.5.). Retrieved from https://www.ncbi.nlm.nih.gov/books/NBK343672

Slof-Op't Landt, M. C., Dingemans, A. E., de la Torre, Y., Rivas, J., \& van Furth, E. F. (2019). Self-assessment of eating disorder recovery: Absence of eating disorder psychopathology is not essential. International Journal of Eating Disorders, 52, 956-961.

Smink, F. R.E., van Hoeken, D., Oldehinkel, A. J. and Hoek, H. W. (2014), Prevalence and severity of DSM-5 eating disorders in a community cohort of adolescents. International Journal of Eating Disorders, 47: 610-619.

Smith, B., \& Sparkes, A. C. (2006). Narrative inquiry in psychology: Exploring the tensions within. Qualitative Research in Psychology, 3, 169-192. https://doi.org/10.1191/1478088706qrp068oa 
Smith, J. D. (2012). Single-case experimental designs: A systematic review of published research and current standards. Psychological Methods, 17(4), 510. https://doi.org/10.1037/a0029312

Solomon, J., \& George, C. (2016). The measurement of attachment security and related constructs in infancy and early childhood. In J. Cassidy \& P. R. Shaver (Eds.), Handbook of Attachment: Theory, research, and clinical applications (3rd ed., pp. 366-396). Guilford Press.

Sprenkle, D. H., Davis, S. D., Lebow, J. L., \& Lebow, J. (2009). Common Factors in Couple and Family Therapy: The Overlooked Foundation for Effective Practice. Guilford Publications.

Stein, H., Jacobs, N. J., Ferguson, K. S., Allen, J. G., \& Fonagy, P. (1998). What do adult attachment scales measure?. Bulletin of the Menninger Clinic, 62(1), 33.

Steinglass, P., Bennett, L. A., Wolin, S. J., \& Reiss, D. (1987). The alcoholic family (pp. xvi, 381). Basic Books.

Stewart, C., Voulgari, S., Eisler, I., Hunt, K. and Simic, M. (2015). Multi-family therapy for bulimia nervosa in adolescence. Eating Disorders, 23(4), pp.345355.

Stice, E., South, K., \& Shaw, H. (2012). Future directions in etiologic, prevention, and treatment research for eating disorders. Journal of Clinical Child and Adolescent $\quad$ Psychology, $\quad 41(6), \quad 845-855$. https://doi.org/10.1080/15374416.2012.728156

Stierlin, H. (1983). Family Therapy-A Science or an Art? Family Process, 22(4), 413-423. https://doi.org/10.1111/j.1545-5300.1983.00413.x

Symons, D. K., Peterson, C. C., Slaughter, V., Roche, J., \& Doyle, E. (2005). Theory of mind and mental state discourse during book reading and story-telling tasks. 
British Journal of Developmental Psychology, 23(1), 81-102. https://doi.org/10.1348/026151004X21080

Szmukler, G. I., Eisler, I., Russell, G. F., \& Dare, C. (1985). Anorexia nervosa, parental "expressed emotion" and dropping out of treatment. The British Journal of Psychiatry, 147, 265-271. https://doi.org/10.1192/bjp.147.3.265

Tasca, G. A., \& Balfour, L. (2014). Attachment and eating disorders: A review of current research. International Journal of Eating Disorders, 47, 710-717.

Tasca, G. A., Ritchie, K., \& Balfour, L. (2011). Implications of attachment theory and research for the assessment and treatment of eating disorders. Psychotherapy, 48(3), 249-259. https://doi.org/10.1037/a0022423

Tasca, G. A., Szadkowski, L., Illing, V., Trinneer, A., Grenon, R., Demidenko, N., Krysanski, V., Balfour, L., \& Bissada, H. (2009). Adult attachment, depression, and eating disorder symptoms: The mediating role of affect regulation strategies. Personality and Individual Differences, 47(6), 662-667. https://doi.org/10.1016/j.paid.2009.06.006

Tchanturia, K., Dapelo, M. A. M., Harrison, A., \& Hambrook, D. (2015). Why study positive emotions in the context of eating disorders? Current Psychiatry Reports, 17(1), 537.

Treasure, J. (2013). Coherence and other autistic spectrum traits and eating disorders: Building from mechanism to treatment. The Birgit Olsson lecture. Nordic $\begin{array}{llll}\text { Journal of } & \text { Psychiatry, }\end{array}$ https://doi.org/10.3109/08039488.2012.674554

Treasure, J., \& Cardi, V. (2017). Anorexia Nervosa, Theory and Treatment: Where Are We 35 Years on from Hilde Bruch's Foundation Lecture? European Eating Disorders Review, 25(3), 139-147. https://doi.org/10.1002/erv.2511 
Tukiewicz, G., Pinzon, V., Lock, J., \& Fleitlich-Bilyk, B. (2010). Feasibility, acceptability, and effectiveness of family-based treatment for adolescent anorexia nervosa: An observational study conducted in Brazil. Revista Brasileira de Psiguiatria, 32, 169-172.

Turnbull, S., Ward, A., Treasure, J., Jick, H., \& Derby, L. (1996). The demand for eating disorder care. An epidemiological study using the general practice research database. British Journal of Psychiatry, 169, 705-712

Ty, M., \& Francis, A. J. P. (2013). Insecure attachment and disordered eating in women: The mediating processes of social comparison and emotion dysregulation. Eating Disorders, 21(2), 154-174. https://doi.org/10.1080/10640266.2013.761089

Vall, E., \& Wade, T. D. (2015). Predictors of treatment outcome in individuals with eating disorders: A systematic review and meta-analysis. International Journal of Eating Disorders, 48(7), 946-971. https://doi.org/10.1002/eat.22411

Van Durme, K., Braet, C., \& Goossens, L. (2015). Insecure Attachment and Eating Pathology in Early Adolescence: Role of Emotion Regulation. The Journal of Early Adolescence, 35(1), 54-78. https://doi.org/10.1177/0272431614523130

Van Durme, K., Goossens, L., Bosmans, G., \& Braet, C. (2018). The Role of Attachment and Maladaptive Emotion Regulation Strategies in the Development of Bulimic Symptoms in Adolescents. Journal of Abnormal Child Psychology, 46(4), 881-893. https://doi.org/10.1007/s10802-017-0334$\underline{1}$

Vaughn, C., \& Leff, J. (1976). The Measurement of Expressed Emotion in the Families of Psychiatric Patients. British Journal of Social and Clinical Psychology, 15(2), 157-165. https://doi.org/10.1111/j.2044-8260.1976.tb00021.x 
Venta, A., Shmueli-Goetz, Y., \& Sharp, C. (2014). Assessing attachment in adolescence: A psychometric study of the Child Attachment Interview. Psychological Assessment, 26(1), 238-255. https://doi.org/10.1037/a0034712

Vet, H. C. W. de, Terwee, C. B., Mokkink, L. B., \& Knol, D. L. (2011). Measurement in Medicine: A Practical Guide. Cambridge University Press.

Von Bertalanffy, L. (1972). The History and Status of General Systems Theory. Academy of Management Journal, 15(4), 407-426. https://doi.org/10.5465/255139

Voriadaki, T., Simic, M., Espie, J., \& Eisler, I. (2015) Intensive multi-family therapy for adolescent anorexia nervosa: Adolescents' and parents' day to day experiences. Journal of Family Therapy, 37, 5-23.

Wade, T. D., \& Lock, J. (2019). Developing consensus on the definition of remission and recovery for research. International Journal of Eating Disorders. https://doi.org/10.1002/eat.23165

Wallis, A., Miskovic-Wheatley, J., Madden, S., Rhodes, P., Crosby, R. D., Cao, L., \& Touyz, S. (2017b). How does family functioning effect the outcome of family based treatment for adolescents with severe anorexia nervosa? Journal of Eating Disorders, 5(1), 55. https://doi.org/10.1186/s40337-017-0184-9

Wallis, A., Miskovic-Wheatley, J., Madden, S., Rhodes, P., Crosby, R. D., Cao, L., \& Touyz, S. (2018). Family Functioning and Relationship Quality for Adolescents in Family-based Treatment with Severe Anorexia Nervosa Compared with Non-clinical Adolescents. European Eating Disorders Review, 26(1), 29-37. https://doi.org/10.1002/erv.2562

Wallis, A., Rhodes, P., Dawson, L., Miskovic-Wheatley, J., Madden, S., \& Touyz, S. (2017a). Relational containment: Exploring the effect of family-based 
treatment for anorexia on familial relationships. Journal of Eating Disorders, 5(1), 27. https://doi.org/10.1186/s40337-017-0156-0

Wallis, A., Rhodes, P., Kohn, M., \& Madden, S. (2007). Five-years of family based treatment for anorexia nervosa: The Maudsley model at the Children's Hospital at Westmead. International Journal of Adolescent Medicine and Health, 19, $277-283$.

Wampold, B.E. (2010). The research evidence for the common factors models: a historically situated perspective. In M. A. Hubble, B. L. Duncan, \& S. D. Miller (Eds.), The Heart and Soul of Change (2 ${ }^{\text {nd }}$ Edition) (pp. 49-82). American Psychological Association.

Ward, A., Ramsay, R., Turnbull, S., Benedettini, M., \& Treasure, J. (2000). Attachment patterns in eating disorders: Past in the present. The International Journal of Eating Disorders, 28(4), 370-376. https://doi.org/10.1002/1098$\underline{108 x(200012) 28: 4<370:: a i d-e a t 4>3.0 . c 0 ; 2-p}$

Ward, A., Ramsay, R., Turnbull, S., Steele, M., Steele, H., \& Treasure, J. (2001). Attachment in anorexia nervosa: A transgenerational perspective. The British Journal of Medical Psychology, 74(4), 497-505. https://doi.org/10.1348/000711201161145

Waters, T. E., Fraley, R. C., Groh, A. M., Steele, R. D., Vaughn, B. E., Bost, K. K., ... \& Roisman, G. I. (2015). The latent structure of secure base script knowledge. Developmental Psychology, 51, 823.

Watson, H.J. \& Bulik, C.M. (2013). Update on the treatment of anorexia nervosa: review of clinical trials, practice guidelines and emerging interventions. Psychological Medicine, 43(12), 2477-2500. 
Weissman, R. S. (2020). Introduction to the Special Issue on Recovery. International Journal of Eating Disorders, 53(8), 1187-1187. https://doi.org/10.1002/eat.23344

Wentz, E., Gillberg, I. C., Anckarsäter, H., Gillberg, C., \& Råstam, M. (2009). Adolescent-onset anorexia nervosa: 18-year outcome. The British Journal of Psychiatry: The Journal of Mental Science, 194(2), 168-174. https://doi.org/10.1192/bjp.bp.107.048686

Westwood, H., \& Tchanturia, K. (2017). Autism spectrum disorder in anorexia nervosa: An updated literature review. Current Psychiatry Reports, 19(7), 41.

Westwood, H., Mandy, W., Simic, M., \& Tchanturia, K. (2018). Assessing ASD in Adolescent Females with Anorexia Nervosa using Clinical and Developmental Measures: A Preliminary Investigation. Journal of Abnormal Child Psychology, 46(1), 183-192. https://doi.org/10.1007/s10802-017-0301-x

Wetzler, S., Hackmann, C., Peryer, G., Clayman, K., Friedman, D., Saffran, K., Silver, J., Swarbrick, M., Magill, E., Furth, E. F. van, \& Pike, K. M. (2020). A framework to conceptualize personal recovery from eating disorders: A systematic review and qualitative meta-synthesis of perspectives from individuals with lived experience. International Journal of Eating Disorders, 53(8), 1188-1203. https://doi.org/10.1002/eat.23260

White, H. J., Haycraft, E., Williamson, I., \& Meyer, C. (2020). Disturbance at the dinner table: Exploring mothers' experiences of mealtimes when caring for their son or daughter with anorexia nervosa. Journal of Health Psychology, 1359105320904756. https://doi.org/10.1177/1359105320904756

White, M. K., \& Epston, D. (1990). Narrative means to therapeutic ends. Norton. 
Wichstrøm, L., Belsky, J., \& Steinsbekk, S. (2017). Homotypic and heterotypic continuity of symptoms of psychiatric disorders from age 4 to 10 years: A dynamic panel model. Journal of Child Psychology and Psychiatry, 58(11), 1239-1247. https://doi.org/10.1111/jepp.12754

Wiener, N. (1948). Cybernetics or control and communication in the animal and the machine. Technology Press.

Williams, A. J., Nielsen, E., \& Coulson, N. S. (2018). "They aren't all like that": Perceptions of clinical services, as told by self-harm online communities. Journal of Health Psychology. https://doi.org/10.1177/1359105318788403

Williams, P., Hand, D., \& Tarnopolsky, A. (1982). The problem of screening for uncommon disorders - a comment on the Eating Attitudes Test. Psychological Medicine, 12(2), 431-434. https://doi.org/10.1017/S003329170004678X

Wilson, J. M., \& Wilkinson, R. B. (2012). The self-report assessment of adolescent attachment: A systematic review and critique. Journal of Relationships Research, 3, 81-94.

Woerwag-Mehta, S., \& Treasure, J. (2008). Causes of anorexia nervosa. Psychiatry, 7(4), 147-151. https://doi.org/10.1016/j.mppsy.2008.02.010

Wood, A., Waller, G., Miller, J., \& Slade, P. (1992). The development of eating attitude test scores in adolescence. International Journal of Eating Disorders, 11(3), 279-282. https://doi.org/10.1002/1098-108X(199204)11:3<279::AID$\underline{\text { EAT2260110312>3.0.CO;2-O }}$

World Health Organization. (2014). Health for the world's adolescents: A second chance in the second decade. World Health Organization. https://www.who.int/maternal_child_adolescent/documents/seconddecade/en/ 
Wright, J. C., Binney, V., \& Smith, P. K. (1995). Security of Attachment in 8-12Year-Olds: A Revised Version of the Separation Anxiety Test, its Psychometric Properties and Clinical Interpretation. Journal of Child Psychology and Psychiatry, 36(5), 757-774. https://doi.org/10.1111/j.1469$\underline{\text { 7610.1995.tb01327.x }}$

Wright, N., Hill, J., Sharp, H. and Pickles, A. (2018), Maternal sensitivity to distress, attachment and the development of callous-unemotional traits in young children. Journal of Child Psychology and Psychiatry. Available on Early View: DOI:10.1111/jepp.12867

Zachrisson, H. D., \& Skårderud, F. (2010). Feelings of insecurity: Review of attachment and eating disorders. European Eating Disorders Review: The Journal of the Eating Disorders Association, 18(2), 97-106. https://doi.org/10.1002/erv.999

Zipfel, S., Giel, K. E., Bulik, C. M., Hay, P., \& Schmidt, U. (2015). Anorexia nervosa: Aetiology, assessment, and treatment. The Lancet Psychiatry, 2(12), 10991111. https://doi.org/10.1016/S2215-0366(15)00356-9

Zonnevijlle-Bender, M. J. S., van Goozen, S. H. M., Cohen-Kettenis, P. T., van Elburg, A., \& van Engeland, H. (2002). Do adolescent anorexia nervosa patients have deficits in emotional functioning? European Child \& Adolescent Psychiatry, 11(1), 38-42. https://doi.org/10.1007/s007870200006

Zonnevylle-Bender, M. J. S., Goozen, S. H. M. van, Cohen-Kettenis, P. T., Elburg, A. van, Wildt, M. de, Stevelmans, E., \& Engeland, H. van. (2004). Emotional functioning in anorexia nervosa patients: Adolescents compared to adults. Depression and Anxiety, 19(1), 35-42. https://doi.org/10.1002/da.10145 


\section{Appendices}

\section{Contents of appendices:}

Appendix A: Characteristics of included studies - Observer-rated measures

Appendix B: Characteristics of included studies - Self-report measures

Appendix C: Methodological quality and adequacy of psychometric properties - Observer-

rated measures

Appendix D: Methodological quality and adequacy of psychometric properties - Self-report

measures

Appendix E: COSMIN criteria for evidence synthesis

Appendix F: Reference list of studies included in the review

Appendix G: Parent Exposure Variables by parental gender

Appendix H: Adolescent self-reported baseline and exposure variables by gender

Appendix I: Means and Standard Deviations of Baseline Variables and Self-Report

Measures by Site

Appendix J: Associations between parent exposure, mediator and outcome variables

Appendix K: Associations between adolescent exposure, mediator and outcome variables 
Appendix A: Characteristics of included studies - Observer-rated measures

\begin{tabular}{|c|c|c|c|c|c|c|c|c|c|}
\hline Instrument & $\begin{array}{l}\text { First author and } \\
\text { year }\end{array}$ & $\mathbf{n}$ & $\begin{array}{l}\text { Age in years } \\
\text { Mean (SD) }\end{array}$ & $\begin{array}{l}\% \\
\text { Male }\end{array}$ & $\begin{array}{l}\text { Clinical or } \\
\text { non-clinical sample }\end{array}$ & Country & Language & Sampling & $\begin{array}{l}\% \\
\text { Response } \\
\text { rate }\end{array}$ \\
\hline$\overline{\text { AAI }}$ & Manassis 1999 & 130 & $15.3(1.47)$ & 56.1 & Clinical & Canada & English & Convenience & NR \\
\hline AAP & Gander 2016 & 79 & $16.78(1.03)$ & 26.6 & Clinical & Austria & German & Convenience & NR \\
\hline AAPQ & Westen 2006 & 150 & $15.6(1.6)$ & 56.0 & Clinical & USA & English & Convenience & $33 \%$ \\
\hline AAQ & Westen 2006 & 125 & $15.6(1.6)$ & 37.5 & Clinical & USA & English & Convenience & $33 \%$ \\
\hline \multirow[t]{2}{*}{ ASCT } & Kerns 2007 & 52 & $10.5(\mathrm{NR})$ & 50.0 & Non-clinical & USA & English & Convenience & NR \\
\hline & Kerns 2011 & 87 & $11.32(.68)$ & 44.8 & $95 \%$ Non-clinical & USA & English & Convenience & 77 \\
\hline \multirow{2}{*}{ ASA } & Steele 2014 & 673 & $18(\mathrm{NR})$ & 52.0 & Non-clinical & USA & English & Random & 49 \\
\hline & Waters 2015 & 674 & $18(\mathrm{NR})$ & 52.0 & Non-clinical & USA & English & Random & 49 \\
\hline BND & Goldner 2014 & 81 & $10.26(1.26)$ & 40.7 & Non-clinical & Israel & Hebrew & Convenience & NR \\
\hline CYTM & Farnfield 2014 & 41 & $9.8(\mathrm{NR})$ & 53.7 & Clinical and non-clinical & UK & English & Convenience & NR \\
\hline CAPA & Farnfield 2016 & 41 & 9.8 (NR) & 53.7 & Clinical and non-clinical & UK & English & Convenience & NR \\
\hline \multirow[t]{7}{*}{ CAI } & Borelli 2016a & 125 & $9.8(1.46)$ & 47.2 & Non-clinical & USA & English & Convenience & NR \\
\hline & Borelli 2016b & 104 & $9.8(1.46)$ & 48.1 & Non-clinical & USA & English & Convenience & NR \\
\hline & Shmueli-Goetz & 161 & $10.9(1.9)$ & 50.3 & Non-clinical & UK & English & Convenience & $55 \%$ \\
\hline & 2008 & 65 & $10.4(1.2)$ & 58.5 & Clinical & UK & English & Convenience & $73 \%$ \\
\hline & & 65 & $10.2(1.3)$ & 58.5 & Clinical & & & & \\
\hline & Venta 2014 & 194 & $15.97(1.4)$ & 40.7 & Clinical & USA & English & Consecutive & NR \\
\hline & Zachrisson 2011 & 150 & $11.7(1.3)$ & 40.0 & Non-clinical & Norway & Norwegian & Convenience & 27.4 \\
\hline \multirow[t]{2}{*}{ CMSSB } & Minnis 2006 & 34 & $7.47(2.09)$ & 62.5 & Clinical & UK & English & Convenience & $63 \%$ \\
\hline & & & $8.94(2.66)$ & 40.0 & Non-clinical & & & & NR \\
\hline \multirow[t]{2}{*}{ CMCAST } & Minnis 2010 & 55 & $6.8(1.4)$ & 66.0 & Clinical and non-clinical & UK & English & Convenience & 87 and 30 \\
\hline & & 58 & $7.46(.99)$ & 56.0 & Non-clinical & & & & 40 \\
\hline
\end{tabular}




\begin{tabular}{|c|c|c|c|c|c|c|c|c|c|}
\hline Instrument & $\begin{array}{l}\text { First author and } \\
\text { year }\end{array}$ & $\mathbf{n}$ & $\begin{array}{l}\text { Age in years } \\
\text { Mean (SD) }\end{array}$ & $\begin{array}{l}\% \\
\text { Male }\end{array}$ & $\begin{array}{l}\text { Clinical or } \\
\text { non-clinical sample }\end{array}$ & Country & Language & Sampling & $\begin{array}{l}\% \\
\text { Response } \\
\text { rate }\end{array}$ \\
\hline FAI & Scharfe 2002 & 127 & $13.6(1.4)$ & 60.6 & Clinical & Canada & English & Convenience & NR \\
\hline \multirow[t]{2}{*}{ FFI } & Stievenart 2012 & 35 & $13.29(1.87)$ & 57.1 & Clinical & Belgium & NR & Convenience & NR \\
\hline & & 26 & $12.91(1.66)$ & 29.5 & Clinical & Romania & NR & Convenience & NR \\
\hline GPACS & Kobac 2017 & 186 & $13.2(\mathrm{NR})$ & 47.3 & Non-clinical & USA & English & Convenience & NR \\
\hline \multirow{4}{*}{ MCAST } & Barone 2009 & 230 & $6.73(1.16)$ & 50.0 & Non-clinical & Italy & Italian & Convenience & NR \\
\hline & Green 2000 & 53 & $6.3(\mathrm{NR})$ & 47.2 & Non-clinical & UK & English & Convenience & NR \\
\hline & Goldwyn 2000 & 53 & $6.3(\mathrm{NR})$ & 47.2 & Non-clinical & UK & English & Convenience & NR \\
\hline & Wan 2017 & 73 & $7.27(.9)$ & 47.9 & Non-clinical & Ghana & English & Convenience & NR \\
\hline \multirow[t]{3}{*}{ SAA } & Crittenden 2010 & 91 & $9.5(\mathrm{NR})$ & 49.5 & Clinical and non-clinical & Australia & English & Convenience & NR \\
\hline & Crittenden 2015 & 50 & $\sim 6.5(\mathrm{NR})$ & 54.0 & Clinical and non-clinical & UK & English & Convenience & NR \\
\hline & Kidwell 2015 & 21 & $12(1.04)$ & 52.0 & Non-clinical & USA & English & Convenience & $39 \%$ \\
\hline \multirow[t]{2}{*}{ SBST } & Psouni 2014 & 141 & $9.8(.65)$ & 49.6 & Non-clinical & Sweden & Swedish & Convenience & NR \\
\hline & & 120 & $10(.91)$ & 44.2 & Non-clinical & Sweden & Swedish & Convenience & NR \\
\hline \multirow[t]{4}{*}{ SAT } & Wright 1990 & 21 & $10(\mathrm{NR})$ & 71.0 & Clinical & UK & English & Convenience & NR \\
\hline & & 21 & $9.7(\mathrm{NR})$ & 71.0 & Non-clinical & UK & English & Convenience & NR \\
\hline & Kerns 2000 & 104 & $9.1(.45)$ & 51.0 & Non-clinical & USA & English & Convenience & NR \\
\hline & & 72 & $12.1(.5)$ & 45.8 & Non-clinical & USA & English & Convenience & NR \\
\hline
\end{tabular}


Appendix B: Characteristics of included studies - Self-report measures

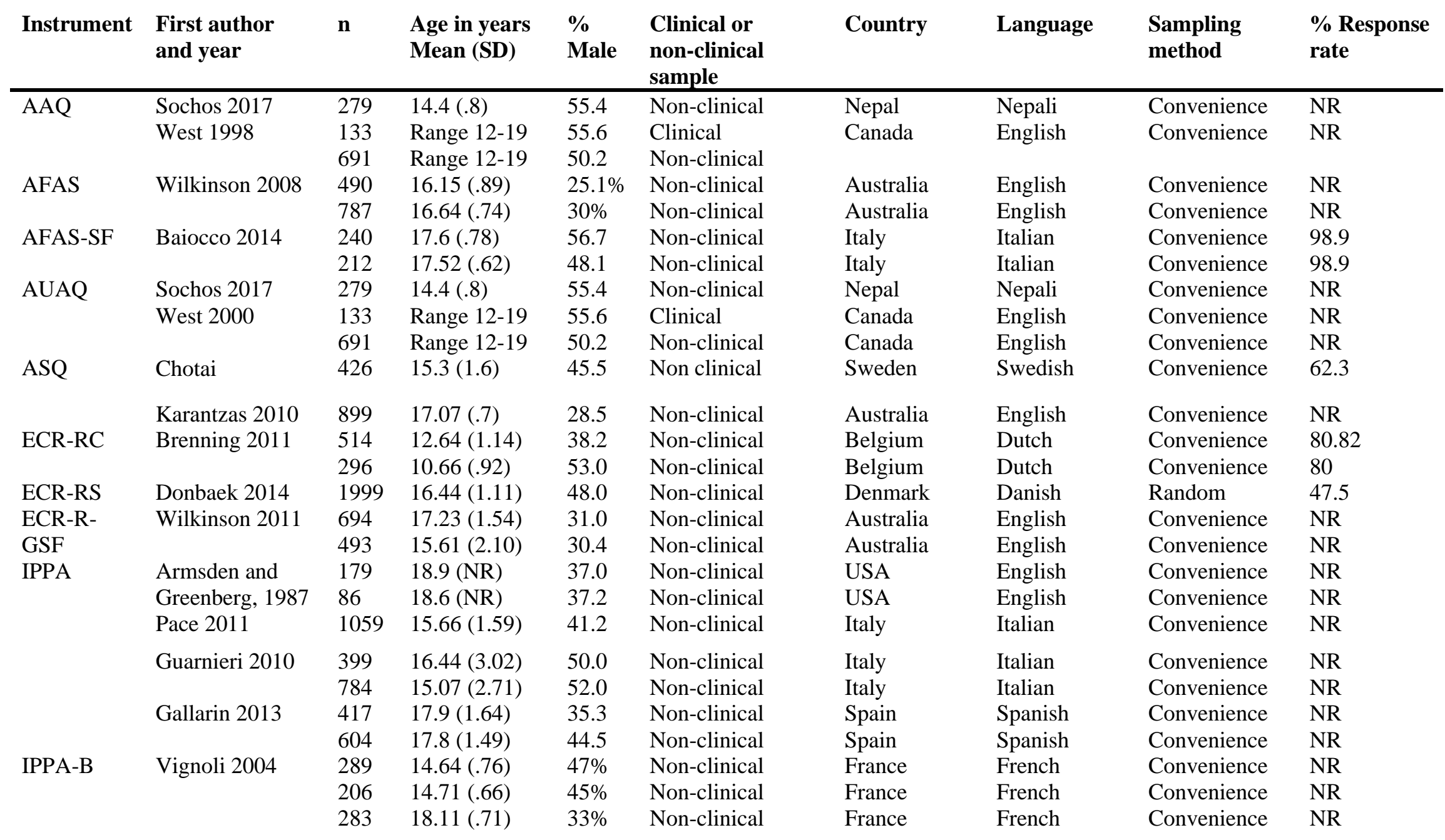




\begin{tabular}{|c|c|c|c|c|c|c|c|c|c|}
\hline Instrument & $\begin{array}{l}\text { First author } \\
\text { and year }\end{array}$ & $\mathbf{n}$ & $\begin{array}{l}\text { Age in years } \\
\text { Mean (SD) }\end{array}$ & $\begin{array}{l}\% \\
\text { Male }\end{array}$ & $\begin{array}{l}\text { Clinical or } \\
\text { non-clinical } \\
\text { sample }\end{array}$ & Country & Language & $\begin{array}{l}\text { Sampling } \\
\text { method }\end{array}$ & $\begin{array}{l}\% \text { Response } \\
\text { rate }\end{array}$ \\
\hline IPPA-45 & Wilkinson 2014 & 1025 & $16.79(.86)$ & 37.8 & Non-clinical & Australia & English & Convenience & NR \\
\hline \multirow{3}{*}{ IPPA-R } & Gullone 2005 & 118 & $9.97(.72)$ & 77.1 & Non-clinical & USA & English & Convenience & 38.76 \\
\hline & & 163 & $14.16(.37)$ & 20.2 & Non-clinical & USA & English & Convenience & \\
\hline & Andretta 2017 & 1126 & Range $12-16$ & 61.0 & Non-clinical & UK & English & Convenience & NR \\
\hline IPPA-S & Andretta 2015 & 213 & $16.17(1.44)$ & 71.0 & Clinical & USA & English & Convenience & 93.8 \\
\hline \multirow[t]{4}{*}{ PACQ } & Finnegan 1996 & 229 & $11.2(\mathrm{NR})$ & 52.0 & Non-clinical & USA & English & Convenience & 92 \\
\hline & Kerns 2000 & 104 & $9.1(.45)$ & 51.0 & Non-clinical & USA & English & Convenience & NR \\
\hline & & 72 & $12.1(.5)$ & 45.8 & & & & & \\
\hline & Kerns 2011 & 87 & $11.32(.68)$ & 44.8 & $\begin{array}{l}\text { Non-clinical } \\
(95 \%)\end{array}$ & USA & English & Convenience & 77 \\
\hline PIML & Ridenour 2006 & 320 & $11.02(.79)$ & 57.8 & Non-clinical & USA & English & Convenience & 74 \\
\hline \multirow[t]{5}{*}{ SS } & Kerns 2000 & 104 & $9.1(.45)$ & 51.0 & Non-clinical & USA & English & Convenience & NR \\
\hline & & 72 & $12.1(.5)$ & 45.8 & & & & & \\
\hline & Kerns 2007 & 52 & $10.5(\mathrm{NR})$ & 50.0 & Non-clinical & USA & English & Convenience & NR \\
\hline & Kerns 2011 & 87 & $11.32(.68)$ & 44.8 & $\begin{array}{l}\text { Non-clinical } \\
(95 \%)\end{array}$ & USA & English & Convenience & 77 \\
\hline & Van Ryzin 2012 & 373 & $\sim 14(\mathrm{NR})$ & NR & Non-clinical & USA & English & Convenience & 97 \\
\hline
\end{tabular}


Appendix C: Methodological quality and adequacy of psychometric properties - Observer-rated measures

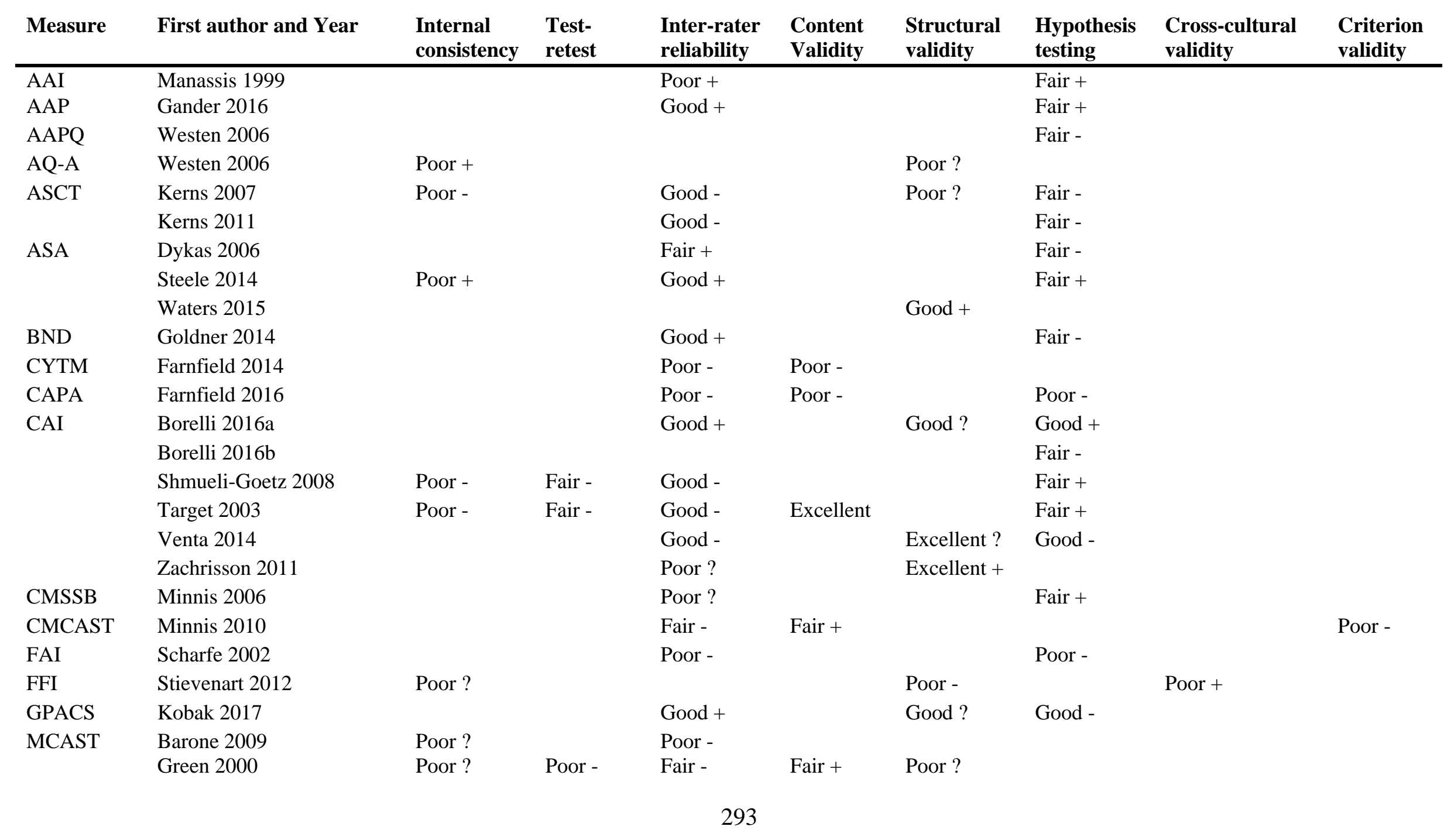




\begin{tabular}{|c|c|c|c|c|c|c|c|c|c|}
\hline Instrument & First author and Year & $\begin{array}{l}\text { Internal } \\
\text { consistency }\end{array}$ & $\begin{array}{l}\text { Test- } \\
\text { retest }\end{array}$ & $\begin{array}{l}\text { Inter-rater } \\
\text { reliability }\end{array}$ & $\begin{array}{l}\text { Content } \\
\text { Validity }\end{array}$ & $\begin{array}{l}\text { Structural } \\
\text { validity }\end{array}$ & $\begin{array}{l}\text { Hypothesis } \\
\text { testing }\end{array}$ & $\begin{array}{l}\text { Cross-cultural } \\
\text { validity }\end{array}$ & $\begin{array}{l}\text { Criterion } \\
\text { validity }\end{array}$ \\
\hline & $\begin{array}{l}\text { Goldwyn } 2000 \\
\text { Wan } 2017\end{array}$ & Fair - & & Fair - & & Fair? & $\begin{array}{l}\text { Fair - } \\
\text { Poor - }\end{array}$ & Poor ? & \\
\hline SAA & $\begin{array}{l}\text { Crittenden } 2010 \\
\text { Crittenden } 2015 \\
\text { Kidwell } 2015\end{array}$ & & & $\begin{array}{l}\text { Poor - } \\
\text { Poor - } \\
\text { Poor? }\end{array}$ & Poor + & & $\begin{array}{l}\text { Poor - } \\
\text { Poor }+ \\
\text { Poor - }\end{array}$ & & \\
\hline $\begin{array}{l}\text { SBST } \\
\text { SAT }\end{array}$ & $\begin{array}{l}\text { Psouni } 2014 \\
\text { Wright } 1995 \\
\text { Kerns } 2000\end{array}$ & $\begin{array}{l}\text { Poor ? } \\
\text { Poor - } \\
\text { Poor - }\end{array}$ & Poor? & $\begin{array}{l}\text { Good + } \\
\text { Poor - }\end{array}$ & Poor + & & $\begin{array}{l}\text { Good - } \\
\text { Poor + } \\
\text { Fair - }\end{array}$ & & \\
\hline
\end{tabular}


Appendix D: Methodological quality and adequacy of psychometric properties - Self-report measures

\begin{tabular}{|c|c|c|c|c|c|c|c|c|c|}
\hline Measure & First author and Year & $\begin{array}{l}\text { Internal } \\
\text { consistency }\end{array}$ & $\begin{array}{l}\text { Test- } \\
\text { retest }\end{array}$ & $\begin{array}{l}\text { Inter-rater } \\
\text { reliability }\end{array}$ & $\begin{array}{l}\text { Content } \\
\text { Validity }\end{array}$ & $\begin{array}{l}\text { Structural } \\
\text { validity }\end{array}$ & $\begin{array}{l}\text { Hypothesis } \\
\text { testing }\end{array}$ & $\begin{array}{l}\text { Cross-cultural } \\
\text { validity }\end{array}$ & $\begin{array}{l}\text { Criterion } \\
\text { validity }\end{array}$ \\
\hline AAQ & $\begin{array}{l}\text { Sochos } 2017 \\
\text { West, } 1998\end{array}$ & $\begin{array}{l}\text { Excellent - } \\
\text { Poor - }\end{array}$ & Fair - & & Excellent & Fair - & Poor? & & \\
\hline AFAS & Wilkinson 2008 & Excellent + & Good + & & Excellent - & Good - & & & \\
\hline AFAS - & Baiocco 2014 & Fair + & & & Fair - & Fair + & & Fair + & \\
\hline AUAQ & $\begin{array}{l}\text { Sochos } 2017 \\
\text { West } 2000\end{array}$ & $\begin{array}{l}\text { Excellent - } \\
\text { Poor - }\end{array}$ & Fair? & Fair + & Excellent & Fair - & Poor? & & \\
\hline ASQ & $\begin{array}{l}\text { Chotai } 2005 \\
\text { Karantzas } 2010\end{array}$ & Excellent + & & & $\begin{array}{l}\text { Excellent } \\
\text { Excellent - }\end{array}$ & & & & \\
\hline $\mathrm{ECR}-\mathrm{RC}$ & Brenning, 2011 & Fair + & & & Fair - & Fair + & & & \\
\hline ECR-RS & Donbaek 2014 & Excellent + & & & Excellent & Fair - & & & \\
\hline ECR-R- & Wilkinson 2011 & Fair + & & Good + & Fair - & Fair - & & & \\
\hline IPPA & $\begin{array}{l}\text { Armsden } 1987 \\
\text { Pace } 2011\end{array}$ & $\begin{array}{l}\text { Poor + } \\
\text { Fair - }\end{array}$ & & Good + & $\begin{array}{l}\text { Poor? } \\
\text { Fair - }\end{array}$ & Fair + & & & \\
\hline & Guarnieri 2010 & Poor? & & & Fair - & Fair + & & & \\
\hline & Gallarin 2013 & Fair + & & & Fair - & Fair + & Poor? & & \\
\hline IPPA-B & Vignoli 2004 & Fair + & & & Fair + & Fair + & Poor? & & \\
\hline IPPA-45 & Wilkinson 2014 & Excellent + & & & Excellent - & & & & \\
\hline IPPA-R & Gullone, 2005 & Fair - & & & & Fair + & & & \\
\hline & Andretta 2017 & Fair - & & & Fair - & Fair + & & & \\
\hline IPPA-S & Andretta 2015 & Fair - & & & Fair - & Fair - & & & \\
\hline PIML & Ridenour 2006 & Fair - & & & Fair + & Fair + & & & \\
\hline PACQ & Finnegan,1996 & Poor - & Fair? & Fair - & & Fair - & & & \\
\hline & Kerns 2000 & Poor + & & & & Fair - & & & \\
\hline & Kerns 2011 & Poor + & & & & Fair - & & & \\
\hline SS & Kerns 2000 & Poor - & & & & Fair - & & & \\
\hline & Kerns 2007 & Poor + & & & & Fair - & & & \\
\hline
\end{tabular}




\begin{tabular}{|c|c|c|c|c|c|c|c|c|c|}
\hline Measure & First author and Year & $\begin{array}{l}\text { Internal } \\
\text { consistency }\end{array}$ & $\begin{array}{l}\text { Test- } \\
\text { retest }\end{array}$ & $\begin{array}{l}\text { Inter-rater } \\
\text { reliability }\end{array}$ & $\begin{array}{l}\text { Content } \\
\text { Validity }\end{array}$ & $\begin{array}{l}\text { Structural } \\
\text { validity }\end{array}$ & $\begin{array}{l}\text { Hypothesis } \\
\text { testing }\end{array}$ & $\begin{array}{l}\text { Cross-cultural } \\
\text { validity }\end{array}$ & $\begin{array}{l}\text { Criterion } \\
\text { validity }\end{array}$ \\
\hline & $\begin{array}{l}\text { Kerns } 2011 \\
\text { Van Ryzin } 2012\end{array}$ & Poor + & & & & $\begin{array}{l}\text { Fair + } \\
\text { Fair - }\end{array}$ & & & \\
\hline
\end{tabular}


Appendix E: COSMIN criteria for evidence synthesis

\begin{tabular}{lll} 
Level & Rating & Criteria \\
\hline Strong & +++ or -- & $\begin{array}{l}\text { Consistent findings in multiple studies of good } \\
\text { methodological quality or in one study of excellent } \\
\text { methodological quality }\end{array}$ \\
Moderate & ++ or -- & $\begin{array}{l}\text { Consistent findings in multiple studies of fair } \\
\text { methodological quality or in one study of good } \\
\text { methodological quality }\end{array}$ \\
Limited & + or - & $\begin{array}{l}\text { One study of fair methodological quality } \\
\text { Conflicting }\end{array}$ \\
Unknown & $+/-$ & Conflicting findings \\
& $?$ & $\begin{array}{l}\text { Indeterminate rating, or only studies of poor } \\
\text { methodological quality }\end{array}$ \\
\hline
\end{tabular}


Appendix F: Reference list of studies included in the review

Andretta, J. R., McKay, M. T., Harvey, S. A., \& Perry, J. L. (2017). Inventory of Parent and Peer Attachment-Revised Scores in Adolescents: A Psychometric and Person-Oriented Study. Family Relations, 66(3), 527-540.

Andretta, J. R., Ramirez, A. M., Barnes, M. E., Odom, T., Roberson-Adams, S., \& Woodland, M. H. (2015). Perceived parental security profiles in African American adolescents involved in the juvenile justice system. Journal of Family Psychology, 29(6), 884.

Armsden, G. C., \& Greenberg, M. T. (1987). The inventory of parent and peer attachment: Individual differences and their relationship to psychological wellbeing in adolescence. Journal of youth and adolescence, 16(5), 427-454.

Baiocco, R., Pallini, S., \& Santamaria, F. (2014). The development and validation of an Italian short form of the adolescent friendship attachment scale. Measurement and Evaluation in Counseling and Development, 47(4), 247-255.

Barone, L., Del Giudice, M., Fossati, A., Manaresi, F., Actis Perinetti, B., Colle, L., \& Veglia, F. (2009). Methods \& measures: Psychometric properties of the Manchester child attachment story task: An Italian multicentre study. International Journal of Behavioral Development, 33(2), 185-190.

Borelli, J. L., Somers, J., West, J. L., Coffey, J. K., De Los Reyes, A., \& ShmueliGoetz, Y. (2016a). Associations between attachment narratives and self-report measures of attachment in middle childhood: Extending evidence for the validity of the Child Attachment Interview. Journal of Child and Family Studies, 25, 1235-1246. 
Borelli, J. L., Somers, J. A., West, J. L., Coffey, J. K., \& Shmueli-Goetz, Y. (2016b). Shedding light on the specificity of school-aged children's attachment narratives. Attachment \& Human Development, 18, 188-211.

Brenning, K., Soenens, B., Braet, C., \& Bosmans, G. (2011). An adaptation of the experiences in close relationships scale-revised for use with children and adolescents. Journal of Social and Personal Relationships, 28, 1048-1072.

Chotai, J., Jonasson, M., Hägglöf, B., \& Adolfsson, R. (2005). Adolescent attachment styles and their relation to the temperament and character traits of personality in a general population. European Psychiatry, 20(3), 251-259.

Crittenden, P., Kozlowska, K., \& Landini, A. (2010). Assessing attachment in schoolage children. Clinical Child Psychology and Psychiatry, 15(2), 185-208.

Crittenden, P., Robson, K., \& Tooby, A. (2015). Validation of the School-age Assessment of Attachment in a short-term longitudinal study. Clinical child psychology and psychiatry, 20(3), 348-365.

Donbaek, D.F., \& Elklit, A. (2014). A validation of the Experiences in Close Relationships-Relationship Structures scale (ECR-RS) in adolescents. Attachment \& human development, 16(1), 58-76.

Dykas, M. J., Woodhouse, S. S., Cassidy, J., \& Waters, H. S. (2006). Narrative assessment of attachment representations: Links between secure base scripts and adolescent attachment. Attachment \& Human Development, 8(3), 221-240.

Farnfield, S. (2014). Assessing attachment in the school years: the application of the Dynamic Maturational Model of Attachment to the coding of a child 
attachment interview with community and looked-after children. Clinical Child Psychology and Psychiatry, 19(4), 516-534.

Farnfield, S. (2016). The Child Attachment and Play Assessment (CAPA): Validation of a new approach to coding narrative stems with children ages 3-11 years. international Journal of play therapy, 25(4), 217.

Finnegan, R. A., Hodges, E. V., \& Perry, D. G. (1996). Preoccupied and avoidant coping during middle childhood. Child Development, 67(4), 1318-1328.

Gallarin, M., \& Alonso-Arbiol, I. (2013). Dimensionality of the inventory of parent and peer attachment: evaluation with the Spanish version. The Spanish Journal of Psychology, 16.

Gander, M., George, C., Pokorny, D., \& Buchheim, A. (2017). Assessing attachment representations in adolescents: discriminant validation of the adult attachment projective picture system. Child Psychiatry \& Human Development, 48(2), 270-282.

Goldner, L. (2014). Revisiting the Bird's Nest Drawing assessment: Toward a global approach. The Arts in Psychotherapy, 41(4), 391-399.

Goldwyn, R., Stanley, C., Smith, V., \& Green, J. (2000). The Manchester child attachment story task: relationship with parental AAI, SAT and child behaviour. Attachment \& Human Development, 2(1), 71-84.

Green, J., Stanley, C., Smith, V., \& Goldwyn, R. (2000). A new method of evaluating attachment representations in young school-age children: The Manchester Child Attachment Story Task. Attachment \& Human Development, 2(1), 4870. 
Guarnieri, S. I. L. V. I. A., Ponti, L. U. C. I. A., \& Tani, F. R. A. N. C. A. (2010). The Inventory of Parent and Peer Attachment (IPPA): A study on the validity of styles of adolescent attachment to parents and peers in an Italian sample. TPMTesting, Psychometrics, Methodology in Applied Psychology, 17(3), 103-130.

Gullone, E., \& Robinson, K. (2005). The inventory of parent and peer attachmentRevised (IPPA-R) for children: a psychometric investigation. Clinical Psychology \& Psychotherapy, 12(1), 67-79.

Karantzas, G. C., Feeney, J. A., \& Wilkinson, R. (2010). Is less more? Confirmatory factor analysis of the Attachment Style Questionnaires. Journal of Social and Personal Relationships, 27(6), 749-780.

Kerns, K. A., Abraham, M. M., Schlegelmilch, A., \& Morgan, T. A. (2007). Motherchild attachment in later middle childhood: Assessment approaches and associations with mood and emotion regulation. Attachment \& Human Development, 9(1), 33-53.

Kerns, K. A., Brumariu, L. E., \& Seibert, A. (2011). Multi-method assessment of mother-child attachment: Links to parenting and child depressive symptoms in middle childhood. Attachment \& Human Development, 13(4), 315-333.

Kerns, K. A., Tomich, P. L., Aspelmeier, J. E., \& Contreras, J. M. (2000). Attachmentbased assessments of parent-child relationships in middle childhood. Developmental Psychology, 36(5), 614.

Kidwell, S. L., Sizemore, K. M., Qu, J., Fugate, K. M., Deaton, M. S., \& Blevins, M. D. (2015). Validity of the School-age Assessment of Attachment for moderate- 
risk, rural early adolescents. Clinical Child Psychology and Psychiatry, 20(3), 366-380.

Kobak, R., Zajac, K., Abbott, C., Zisk, A., \& Bounoua, N. (2017). Atypical dimensions of caregiver-adolescent interaction in an economically disadvantaged sample. Development and Psychopathology, 29(2), 405-416.

Manassis, K., Owens, M., Adam, K. S., West, M., \& Sheldon-Keller, A. E. (1999). Assessing attachment: convergent validity of the adult attachment interview and the parental bonding instrument. Australian \& New Zealand Journal of Psychiatry, 33(4), 559-567.

Minnis, H., Millward, R., Sinclair, C., Kennedy, E., Greig, A., Towlson, K., ... \& Hill, J. (2006). The Computerized MacArthur Story Stem Battery-a pilot study of a novel medium for assessing children's representations of relationships. International journal of methods in psychiatric research, 15(4), 207-214.

Minnis, H., Read, W., Connolly, B., Burston, A., Schumm, T. S., Putter-Lareman, S., \& Green, J. (2010). The Computerised Manchester Child Attachment Story Task: a novel medium for assessing attachment patterns. International Journal of Methods in Psychiatric Research, 19(4), 233-242.

Pace, C. S., San Martini, P., \& Zavattini, G. C. (2011). The factor structure of the Inventory of Parent and Peer Attachment (IPPA): A survey of Italian adolescents. Personality and Individual Differences, 51(2), 83-88.

Psouni, E., \& Apetroaia, A. (2014). Measuring scripted attachment-related knowledge in middle childhood: the Secure Base Script Test. Attachment \& human development, 16(1), 22-41. 
Ridenour, T. A., Greenberg, M. T., \& Cook, E. T. (2006). Structure and validity of people in my life: A self-report measure of attachment in late childhood. Journal of Youth and Adolescence, 35(6), 1037-1053.

Scharfe, E. (2002). Reliability and validity of an interview assessment of attachment representations in a clinical sample of adolescents. Journal of Adolescent Research, 17(5), 532-551.

Shmueli-Goetz, Y., Target, M., Fonagy, P. \& Datta, A. (2008). The Child Attachment Interview: A psychometric study of reliability and discriminant validity. Developmental Psychology, 44, 939.

Sochos, A., \& Lokshum, C. (2017). Adolescent attachment in Nepal: Testing the factorial validity of two scales. European Journal of Developmental Psychology, 14(4), 498-508.

Steele, R. D., Waters, T. E., Bost, K. K., Vaughn, B. E., Truitt, W., Waters, H. S., ... \& Roisman, G. I. (2014). Caregiving antecedents of secure base script knowledge: A comparative analysis of young adult attachment representations. Developmental Psychology, 50(11), 2526.

Stievenart, M., Casonato, M., Muntean, A., \& Van de Schoot, R. (2012). The friends and family interview: measurement invariance across Belgium and Romania. European Journal of Developmental Psychology, 9(6), 737-743.

Target, M., Fonagy, P., \& Shmueli-Goetz, Y. (2003). Attachment representations in school-age children: The development of the Child Attachment Interview (CAI). Journal of Child Psychotherapy, 29, 171-186. 
Tasca, G. A., Szadkowski, L., Illing, V., Trinneer, A., Grenon, R., Demidenko, N., Krysanski, V., Balfour, L. \& Bissada, H. (2009). Adult attachment, depression, and eating disorder symptoms: The mediating role of affect regulation strategies. Personality and Individual Differences, 47(6), 662-667.

Van Ryzin, M. J., \& Leve, L. D. (2012). Validity evidence for the security scale as a measure of perceived attachment security in adolescence. Journal of Adolescence, 35(2), 425-431.

Venta, A., Shmueli-Goetz, Y., \& Sharp, C. (2014). Assessing attachment in adolescence: A psychometric study of the child attachment interview. Psychological Assessment, 26(1), 238-255.

Vignoli, E., \& Mallet, P. (2004). Validation of a brief measure of adolescents' parent attachment based on Armsden and Greenberg's three-dimension model. Revue Européenne de Psychologie Appliquée/European Review of Applied Psychology, 54, 251-260.

Wan, M. W., Danquah, A. N., \& Mahama, S. (2017). Child-caregiver Attachment Representations in a Non-Western Context: The Feasibility and Cultural Equivalence of Story Stems in Urban Ghana. Social Development, 26, 59-79.

Waters, T. E., Fraley, R. C., Groh, A. M., Steele, R. D., Vaughn, B. E., Bost, K. K., ,.. \& Roisman, G. I. (2015). The latent structure of secure base script knowledge. Developmental psychology, 51(6), 823.

West, M., Rose, M. S., Spreng, S., Sheldon-Keller, A., \& Adam, K. (1998). Adolescent attachment questionnaire: A brief assessment of attachment in adolescence. Journal of Youth and adolescence, 27(5), 661-673. 
West, M., Rose, S., Spreng, S., \& Adam, K. (2000). The Adolescent Unresolved Attachment Questionnaire: The assessment of perceptions of parental abdication of caregiving behavior. The Journal of Genetic Psychology, 161(4), 493-503.

Westen, D., Nakash, O., Thomas, C., \& Bradley, R. (2006). Clinical assessment of attachment patterns and personality disorder in adolescents and adults. Journal of consulting and clinical psychology, 74(6), 1065.

Wilkinson, R. B. (2008). Development and properties of the adolescent friendship attachment scale. Journal of Youth and Adolescence, 37(10), 1270-1279.

Wilkinson, R. B. (2011). Measuring attachment dimensions in adolescents: Development and validation of the Experiences in Close RelationshipsRevised-General Short Form. Journal of Relationships Research, 2(1), 5362.

Wilkinson, R. B., \& Goh, D. Y. L. (2014). Structural, age, and sex differences for a short form of the inventory of parent and peer attachment: the IPPA-45. Journal of Relationships Research, 5(e5), 1-11.

Wright, J. C., Binney, V., \& Smith, P. K. (1995). Security of attachment in 8-12-yearolds: a revised version of the Separation Anxiety Test, its psychometric properties and clinical interpretation. Journal of Child Psychology and Psychiatry, 36(5), 757-774.

Zachrisson, H. D., Røysamb, E., Oppedal, B., \& Hauser, S. T. (2011). Factor structure of the child attachment interview. European Journal of Developmental Psychology, 8, 744-759. 
Appendix G: Parent Exposure Variables by parental gender

\begin{tabular}{|c|c|c|c|c|c|}
\hline Exposure variable & $\begin{array}{l}\text { Mother } \\
\text { or father }\end{array}$ & $\mathbf{N}$ & Mean & $\begin{array}{l}\text { Standard } \\
\text { Deviation }\end{array}$ & $\begin{array}{c}\text { Standard } \\
\text { Error Mean }\end{array}$ \\
\hline \multirow[t]{2}{*}{ RFQ8 Certainty (T1) } & M & 149 & 1.2506 & .74928 & .06138 \\
\hline & $\mathrm{F}$ & 14 & .9405 & .63248 & .16904 \\
\hline \multirow[t]{2}{*}{ RFQ8 Uncertainty (T1) } & M & 149 & .4016 & .43712 & .03581 \\
\hline & $\mathrm{F}$ & 14 & .5238 & .54638 & .14603 \\
\hline \multirow[t]{2}{*}{ Hypermentalizing (T1) } & M & 148 & 39.86 & 17.711 & 1.456 \\
\hline & $\mathrm{F}$ & 14 & 38.93 & 16.373 & 4.376 \\
\hline \multirow[t]{2}{*}{ DERS Total (T1) } & M & 149 & 70.16 & 19.067 & 1.562 \\
\hline & $\mathrm{F}$ & 14 & 82.57 & 16.883 & 4.512 \\
\hline \multirow[t]{2}{*}{ DERS Nonacceptance (T1) } & M & 149 & 11.20 & 5.100 & .418 \\
\hline & $\mathrm{F}$ & 14 & 13.71 & 5.121 & 1.369 \\
\hline \multirow[t]{2}{*}{ DERS Goals (T1) } & M & 149 & 12.39 & 4.541 & .372 \\
\hline & $\mathrm{F}$ & 14 & 14.36 & 3.835 & 1.025 \\
\hline \multirow[t]{2}{*}{ DERS Impulse (T1) } & M & 149 & 9.99 & 4.302 & .352 \\
\hline & $\mathrm{F}$ & 14 & 11.79 & 5.056 & 1.351 \\
\hline \multirow[t]{2}{*}{ DERS Awareness (T1) } & M & 149 & 14.31 & 4.416 & .362 \\
\hline & $\mathrm{F}$ & 14 & 16.43 & 3.345 & .894 \\
\hline \multirow[t]{2}{*}{ DERS Strategies (T1) } & M & 149 & 13.66 & 5.430 & .445 \\
\hline & $\mathrm{F}$ & 14 & 15.64 & 5.242 & 1.401 \\
\hline \multirow[t]{2}{*}{ DERS Clarity (T1) } & M & 149 & 8.61 & 2.952 & .242 \\
\hline & $\mathrm{F}$ & 14 & 10.64 & 1.823 & .487 \\
\hline \multirow[t]{2}{*}{ ASQ Confidence (T1) } & M & 149 & 36.30 & 5.652 & .463 \\
\hline & $\mathrm{F}$ & 14 & 33.43 & 4.090 & 1.093 \\
\hline \multirow[t]{2}{*}{ ASQ Secondary (T1) } & M & 149 & 14.30 & 4.678 & .383 \\
\hline & $\mathrm{F}$ & 14 & 17.43 & 2.563 & .685 \\
\hline \multirow[t]{2}{*}{ ASQ Need (T1) } & M & 148 & 20.59 & 5.732 & .471 \\
\hline & $\mathrm{F}$ & 14 & 22.14 & 3.800 & 1.016 \\
\hline \multirow[t]{2}{*}{ ASQ Preoccupation (T1) } & M & 149 & 24.36 & 6.646 & .544 \\
\hline & $\mathrm{F}$ & 14 & 28.00 & 6.139 & 1.641 \\
\hline \multirow[t]{2}{*}{ ASQ Discomfort (T1) } & M & 149 & 31.83 & 8.517 & .698 \\
\hline & $\mathrm{F}$ & 14 & 33.00 & 7.390 & 1.975 \\
\hline \multirow[t]{2}{*}{ SOFTA Engagement (T2) } & M & 117 & 15.91 & 2.683 & .248 \\
\hline & $\mathrm{F}$ & 12 & 14.92 & 1.929 & .557 \\
\hline \multirow[t]{2}{*}{ SOFTA Connection (T2) } & M & 117 & 15.85 & 2.821 & .261 \\
\hline & $\mathrm{F}$ & 12 & 14.92 & 2.999 & .866 \\
\hline \multirow[t]{2}{*}{ SOFTA Safety (T2) } & M & 117 & 15.90 & 2.830 & .262 \\
\hline & $\mathrm{F}$ & 12 & 16.17 & 1.899 & .548 \\
\hline \multirow[t]{2}{*}{ SOFTA Purpose (T2) } & M & 117 & 16.89 & 2.888 & .267 \\
\hline & $\mathrm{F}$ & 12 & 15.92 & 2.610 & .753 \\
\hline
\end{tabular}


Note. Abbreviations in table: M, Mother; F, Father; RFQ8, Reflective Function

Questionnaire (8 item version); RFQ8 Certainty, RFQ8 Certainty About Mental States;

RFQ8 Uncertainty, RFQ8 Uncertainty About Mental States; ASQ, Attachment Style

Questionnaire; ASQ Discomfort, ASQ Discomfort with Closeness; ASQ Preoccupation, ASQ Preoccupation with Relationships; ASQ Secondary, ASQ Relationships as Secondary; DERS, Difficulties in Emotion Regulation Strategies Scale; DERS Nonacceptance, DERS Nonacceptance of Emotional Responses; DERS Goals, DERS Difficulties Engaging in GoalDirected Behaviors; DERS Impulse, DERS Impulse Control Difficulties; DERS Awareness, DERS Lack of Emotional Awareness; DERS Strategies, DERS Limited Access to Effective Emotion Regulation Strategies; DERS Clarity, DERS Lack of Emotional Clarity; Hypermentalizing, Hypermentalizing Questionnaire; SOFTA, System for Observing Family Therapy Alliance; SOFTA Engagement, SOFTA Engagement in the Therapeutic Process; SOFTA Connection, SOFTA Emotional Connection to the Therapist; SOFTA Safety, SOFTA Safety within the Therapeutic System; SOFTA Purpose, SOFTA Shared Sense of Purpose within the Family. 
Appendix H: Adolescent self-reported baseline and exposure variables by gender

\begin{tabular}{|c|c|c|c|c|c|}
\hline $\begin{array}{l}\text { Baseline or Exposure } \\
\text { Variable } \\
\end{array}$ & Gender & $\mathbf{N}$ & Mean & $\begin{array}{l}\text { Standard } \\
\text { Deviation } \\
\end{array}$ & $\begin{array}{c}\text { Standard } \\
\text { Error Mean }\end{array}$ \\
\hline \multirow{2}{*}{ EDEQ Global } & Female & 141 & 3.33 & 1.58 & .13 \\
\hline & Male & 18 & 3.56 & 1.41 & .33 \\
\hline \multirow[t]{2}{*}{ RCADS Total Internalising } & Female & 112 & 56.04 & 27.48 & 2.60 \\
\hline & Male & 15 & 49.07 & 26.30 & 6.79 \\
\hline \multirow[t]{2}{*}{ ASQ Confidence (T1) } & Female & 150 & 29.09 & 7.15 & .58 \\
\hline & Male & 20 & 27.35 & 8.04 & 1.80 \\
\hline \multirow[t]{2}{*}{ YP ASQ Discomfort (T1) } & Female & 150 & 39.69 & 9.00 & .74 \\
\hline & Male & 20 & 40.85 & 7.94 & 1.77 \\
\hline \multirow[t]{2}{*}{ YP ASQ Secondary (T1) } & Female & 150 & 19.39 & 5.98 & .49 \\
\hline & Male & 20 & 21.10 & 5.52 & 1.23 \\
\hline \multirow[t]{2}{*}{ YP ASQ Need (T1) } & Female & 150 & 30.32 & 5.90 & .48 \\
\hline & Male & 20 & 31.30 & 5.13 & 1.15 \\
\hline \multirow[t]{2}{*}{ ASQ Preoccupation (T1) } & Female & 150 & 30.03 & 6.67 & .55 \\
\hline & Male & 20 & 28.25 & 6.86 & 1.53 \\
\hline \multirow{2}{*}{$\begin{array}{l}\text { DERS Nonacceptance } \\
\text { (T1) }\end{array}$} & Female & 150 & 18.24 & 7.42 & .61 \\
\hline & Male & 20 & 21.30 & 8.21 & 1.84 \\
\hline \multirow[t]{2}{*}{ DERS Goals (T1) } & Female & 150 & 17.86 & 5.29 & .43 \\
\hline & Male & 20 & 18.85 & 4.55 & 1.01 \\
\hline \multirow[t]{2}{*}{ DERS Impulse (T1) } & Female & 150 & 17.93 & 6.92 & .57 \\
\hline & Male & 20 & 15.30 & 5.68 & 1.27 \\
\hline \multirow[t]{2}{*}{ DERS Awareness (T1) } & Female & 150 & 17.52 & 5.05 & .41 \\
\hline & Male & 20 & 18.40 & 5.58 & 1.25 \\
\hline \multirow[t]{2}{*}{ DERS Strategies (T1) } & Female & 150 & 25.65 & 8.80 & .72 \\
\hline & Male & 20 & 26.75 & 7.17 & 1.60 \\
\hline \multirow[t]{2}{*}{ DERS Clarity (T1) } & Female & 150 & 14.49 & 5.08 & .42 \\
\hline & Male & 20 & 15.25 & 4.17 & .93 \\
\hline \multirow[t]{2}{*}{ RFQY Total (T1) } & Female & 150 & 8.80 & .78 & .06 \\
\hline & Male & 20 & 8.60 & .84 & .19 \\
\hline \multirow{2}{*}{$\begin{array}{l}\text { Hypermentalizing Total } \\
\text { (T1) }\end{array}$} & Female & 149 & 54.19 & 18.53 & 1.51 \\
\hline & Male & 20 & 48.85 & 17.19 & 3.85 \\
\hline \multirow[t]{2}{*}{ SOFTA Engagement (T2) } & Female & 107 & 12.14 & 3.52 & .34 \\
\hline & Male & 18 & 13.67 & 4.06 & .957 \\
\hline \multirow[t]{2}{*}{ SOFTA Connection (T2) } & Female & 107 & 14.11 & 4.00 & .387 \\
\hline & Male & 18 & 15.72 & 3.80 & .896 \\
\hline \multirow[t]{2}{*}{ SOFTA Safety (T2) } & Female & 107 & 11.31 & 3.65 & .353 \\
\hline & Male & 18 & 12.78 & 3.89 & .917 \\
\hline \multirow[t]{2}{*}{ SOFTA Purpose (T2) } & Female & 107 & 14.99 & 3.20 & .309 \\
\hline & Male & 18 & 15.33 & 4.06 & .957 \\
\hline
\end{tabular}


Note. Abbreviations in table: RCADS, Revised Child Anxiety and Depression Scale (RCADS); EDE-Q, Eating Disorder Examination Questionnaire; ASQ, Attachment Style Questionnaire; ASQ Discomfort, ASQ Discomfort with Closeness; ASQ Preoccupation, ASQ Preoccupation with Relationships; ASQ Secondary, ASQ Relationships as Secondary; DERS, Difficulties in Emotion Regulation Strategies Scale; DERS Nonacceptance, DERS Nonacceptance of Emotional Responses; DERS Goals, DERS Difficulties Engaging in Goal-Directed Behaviors; DERS Impulse, DERS Impulse Control Difficulties; DERS Awareness, DERS Lack of Emotional Awareness; DERS Strategies, DERS Limited Access to Effective Emotion Regulation Strategies; DERS Clarity, DERS Lack of Emotional Clarity; RFQY, Reflective Function Questionnaire - Youth Total Score; SOFTA, System for Observing Family Therapy Alliance; SOFTA Engagement, SOFTA Engagement in the Therapeutic Process; SOFTA Connection, SOFTA Emotional Connection to the Therapist; SOFTA Safety, SOFTA Safety within the Therapeutic System; SOFTA Purpose, SOFTA Shared Sense of Purpose within the Family; RFQ8, Reflective Function Questionnaire (8 item version); RFQ8 Certainty, RFQ8 Certainty About Mental States; RFQ8 Uncertainty, RFQ8 Uncertainty About Mental States. 
Appendix I: Means and Standard Deviations of Baseline Variables and Self-Report Measures by Site

\begin{tabular}{|c|c|c|c|}
\hline & $\begin{array}{l}\text { Site 1 } \\
\text { (Mean, SD) }\end{array}$ & $\begin{array}{l}\text { Site 2 } \\
\text { (Mean, SD) }\end{array}$ & $\begin{array}{l}\text { Site 3 } \\
\text { (Mean, SD) } \\
\end{array}$ \\
\hline \multicolumn{4}{|l|}{ Adolescents } \\
\hline Age (Years) & $14.76(1.60)$ & $14.49(1.33)$ & $14.75(1.60)$ \\
\hline \%median BMI & $84.36(.82)$ & $83.00(1.41)$ & $82.50(2.61)$ \\
\hline EDE-Q Global (T1) & $3.27(.15)$ & $3.55(.23)$ & $3.50(.61)$ \\
\hline RCADS Internalising Total & $55.05(2.70)$ & $55.79(5.56)$ & Not available \\
\hline ASQ Confidence (T1) & $28.99(.65)$ & $29.24(1.31)$ & $26.58(1.08)$ \\
\hline ASQ Discomfort (T1) & $39.66(.77)$ & $40.10(1.58)$ & $40.5(2.80)$ \\
\hline ASQ Secondary (T1) & $19.59(.54)$ & $19.79(1.03)$ & $18.92(1.48)$ \\
\hline ASQ Need for Approval (T1) & $30.26(.53)$ & $30.67(.95)$ & $31.33(1.84)$ \\
\hline ASQ Preoccupation (T1) & $29.45(.64)$ & $29.81(1.03)$ & $33.50(1.19)$ \\
\hline DERS Nonacceptance (T1) & $18.29(.69)$ & $19.90(1.22)$ & $17.00(2.26)$ \\
\hline DERS Goals (T1) & $17.68(.48)$ & $18.36(.82)$ & $19.50(1.52)$ \\
\hline DERS Impulse (T1) & $17.18(.64)$ & $19.07(.99)$ & $16.75(2.22)$ \\
\hline DERS Awareness (T1) & $17.72(.51)$ & $17.79(.64)$ & $16.17(1.31)$ \\
\hline DERS Strategies (T1) & $25.53(.81)$ & $26.57(1.26)$ & $25.50(2.68)$ \\
\hline DERS Clarity (T1) & $14.71(.45)$ & $14.29(.82)$ & $14.33(1.57)$ \\
\hline HMZ Total (T1) & $53.42(1.77)$ & $52.95(2.84)$ & $57.00(3.89)$ \\
\hline RFQY (T1) & $8.86(.07)$ & $8.62(.14)$ & $8.55(.22)$ \\
\hline SOFTA Engagement (T2) & $12.36(.36)$ & $12.65(.84)$ & $11.33(.88)$ \\
\hline SOFTA Connection (T2) & $14.16(.44)$ & $15.06(.76)$ & $13.56(.77)$ \\
\hline SOFTA Safety (T2) & $11.67(.37)$ & $11.39(.83)$ & $10.56(1.12)$ \\
\hline SOFTA Purpose (T2) & $14.85(.36)$ & $15.65(.59)$ & $14.78(1.08)$ \\
\hline \multicolumn{4}{|l|}{ Parents } \\
\hline ASQ Confidence (T1) & $36.03(.55)$ & $36.1(.91)$ & $36.17(.84)$ \\
\hline ASQ Discomfort (T1) & $31.62(.78)$ & $32.33(1.54)$ & $33.50(1.70)$ \\
\hline ASQ Secondary (T1) & $14.98(.43)$ & $13.73(.78)$ & $13.58(1.06)$ \\
\hline ASQ Need (T1) & $20.77(.51)$ & $20.68(1.06)$ & $20.50(1.32)$ \\
\hline ASQ Preoccupation (T1) & $25.06(.64)$ & 24.05 (1.09) & $23.17(1.50)$ \\
\hline DERS Nonacceptance (T1) & $11.48(.48)$ & $11.43(.86)$ & $10.83(1.51)$ \\
\hline DERS Goals (T1) & $12.52(.43)$ & $12.88(.73)$ & $11.83(1.25)$ \\
\hline DERS Impulse (T1) & $10.16(.39)$ & $9.93(.79)$ & $10.75(1.39)$ \\
\hline DERS Awareness (T1) & $14.42(.41)$ & $14.48(.74)$ & $15.17(.98)$ \\
\hline DERS Strategies (T1) & $14.16(.51)$ & $12.78(.89)$ & $14.25(1.60)$ \\
\hline DERS Clarity (T1) & $9.05(.30)$ & $8.10(.40)$ & $8.58(.47)$ \\
\hline RFQ8 Certainty (T1) & $1.25(.06)$ & $1.16(.14)$ & $1.19(.24)$ \\
\hline RFQ8 Uncertainty (T1) & $.38(.04)$ & $.48(.08)$ & $.44(.18)$ \\
\hline HMZ Total (T1) & $40.81(1.68)$ & $39.97(2.75)$ & $29.67(4.52)$ \\
\hline SOFTA Engagement (T2) & $15.62(.28)$ & $16.55(.46)$ & $16.00(.93)$ \\
\hline SOFTA Connection (T2) & $15.49(.28)$ & $16.59(.57)$ & $15.90(.84)$ \\
\hline SOFTA Safety (T2) & $15.75(.28)$ & $16.76(.49)$ & $15.70(.99)$ \\
\hline SOFTA Purpose (T2) & $16.40(.31)$ & $17.90(.39)$ & $17.70(.90)$ \\
\hline
\end{tabular}

Note. Abbreviations in table: EDE-Q Global, Eating Disorder Examination

Questionnaire Global Score; RCADS, Revised Child Anxiety and Depression Scale (RCADS); ASQ, Attachment Style Questionnaire; ASQ Discomfort, ASQ Discomfort 
with Closeness; ASQ Preoccupation, ASQ Preoccupation with Relationships; ASQ Secondary, ASQ Relationships as Secondary; DERS, Difficulties in Emotion Regulation Strategies Scale; DERS Nonacceptance, DERS Nonacceptance of Emotional Responses; DERS Goals, DERS Difficulties Engaging in Goal-Directed Behaviors; DERS Impulse, DERS Impulse Control Difficulties; DERS Awareness, DERS Lack of Emotional Awareness; DERS Strategies, DERS Limited Access to Effective Emotion Regulation Strategies; DERS Clarity, DERS Lack of Emotional Clarity; HMZ Total, Hypermentalizing Questionnaire Total Score; RFQY, Reflective Function Questionnaire - Youth Total Score; SOFTA, System for Observing Family Therapy Alliance; SOFTA Engagement, SOFTA Engagement in the Therapeutic Process; SOFTA Connection, SOFTA Emotional Connection to the Therapist; SOFTA Safety, SOFTA Safety within the Therapeutic System; SOFTA Purpose, SOFTA Shared Sense of Purpose within the Family; RFQ8, Reflective Function Questionnaire (8 item version); RFQ8 Certainty, RFQ8 Certainty About Mental States; RFQ8 Uncertainty, RFQ8 Uncertainty About Mental States. 
Appendix J: Associations between parent exposure, mediator and outcome variables

\begin{tabular}{|c|c|c|c|c|c|c|c|c|c|c|c|c|}
\hline \multirow{4}{*}{ Predictors at baseline } & \multirow{2}{*}{\multicolumn{10}{|c|}{$\overline{\text { Parent Mediator T2 }}$}} & \multirow{3}{*}{\multicolumn{2}{|c|}{$\begin{array}{l}\begin{array}{l}\text { Outcome at end of } \\
\text { treatment }\end{array} \\
\text { Morgan Russell } \\
\text { Outcome }\end{array}$}} \\
\hline & & & & & & & & & & & & \\
\hline & \multicolumn{2}{|c|}{ SOFTA Total } & \multicolumn{2}{|c|}{ SOFTA Engagement } & \multicolumn{2}{|c|}{ SOFTA Connection } & \multicolumn{2}{|c|}{ SOFTA Safety } & \multicolumn{2}{|c|}{ SOFTA Purpose } & & \\
\hline & Beta & $95 \% \mathrm{CI}$ & Beta & $95 \% \mathrm{CI}$ & Beta & $95 \% \mathrm{CI}$ & Beta & $95 \% \mathrm{CI}$ & Beta & $95 \% \mathrm{CI}$ & OR & $95 \% \mathrm{CI}$ \\
\hline ASQ Confidence & .39 & $.08-.70$ & .06 & $-.03-.16$ & .08 & $-.02-.18$ & .17 & $.07-.26$ & .08 & $-.01-.18$ & 1.02 & $.95-1.11$ \\
\hline ASQ Discomfort & .09 & $-.30-.13$ & .00 & $-.06-.07$ & .01 & $-.06-.07$ & -.06 & $-.12-.01$ & -.03 & $-.09-.02$ & 1.01 & $.95-1.08$ \\
\hline ASQ Secondary & -.33 & $-.73-.07$ & -.06 & $-.18-.05$ & -.06 & $-.18-.07$ & -.10 & $-.21-.01$ & -.11 & $-.24-.02$ & 1.02 & $.91-1.14$ \\
\hline ASQ Need for Approval & -.16 & $-.48-.15$ & -.01 & $-.10-.08$ & .03 & $-.07-.13$ & -.10 & $-.19--.01$ & -.09 & $-.18-.00$ & 1.04 & $.94-1.15$ \\
\hline ASQ Preoccupation & -.22 & $-.50-.05$ & -.02 & $-.10-.06$ & -.02 & $-.10-.07$ & -.11 & $-.19--.03$ & -.08 & $-.15--.00$ & 1.03 & $.96-1.10$ \\
\hline DERS Total & -.06 & $-.16-.03$ & -.00 & $-.03-.02$ & .00 & $-.02-.03$ & -.03 & $-.06--.00$ & -.03 & $-.06--.00$ & 1.02 & $.99-1.05$ \\
\hline DERS Nonacceptance & -.14 & $-.48-.20$ & -.00 & $-.10-.09$ & .02 & $-.08-.13$ & -.08 & $-.18-.02$ & -.09 & $-.19-.02$ & 1.09 & $1.00-1.19$ \\
\hline DERS Goals & -.33 & $-.73-.06$ & -.02 & $-.14-.10$ & -.03 & $-.15-.09$ & -.15 & $-.26--.03$ & -.13 & $-.25--.02$ & 1.07 & $.96-1.19$ \\
\hline DERS Impulse & -.18 & $-.61-.26$ & -.01 & $-.13-.11$ & .00 & $-.13-.13$ & -.05 & $-.17-.08$ & -.12 & $-.26-.01$ & 1.23 & $1.07-1.43$ \\
\hline DERS Awareness & -.09 & $-.44-.26$ & -.03 & $-.13-.08$ & .04 & $-.07-.15$ & -.07 & $-.17-.04$ & -.04 & $-.15-.08$ & .93 & $.83-1.05$ \\
\hline DERS Strategies & -.18 & $-.54-.17$ & -.00 & $-.10-.09$ & .00 & $-.10-.11$ & -.10 & $-.21-.01$ & -.08 & $-.19-.03$ & 1.04 & $.94-1.15$ \\
\hline DERS Clarity & -.27 & $-.86-.31$ & .01 & $-.17-.17$ & .07 & $-.11-.25$ & -.13 & $-.31-.04$ & -.22 & $-.38--.04$ & 1.15 & $.97-1.38$ \\
\hline HMZ Total & -.07 & $-.16-.02$ & -.01 & $-.04-.02$ & .00 & $-.03-.03$ & -.03 & $-.05-.00$ & -.04 & $-.07--.01$ & 1.03 & $1.00-1.06$ \\
\hline RFQ8 Certainty & 1.23 & $-1.03-3.50$ & .16 & $-.50-.81$ & -.13 & $-.84-.58$ & .28 & $-.40-.96$ & .93 & $.24-1.62$ & .42 & $.20-.87$ \\
\hline RFQ8 Uncertainty & -1.35 & $-5.32-2.62$ & -.02 & $-1.14-1.10$ & .62 & $-.54-1.78$ & -.81 & $-1.95-.32$ & -1.13 & $-2.32-.05$ & 2.72 & $.78-9.51$ \\
\hline \multicolumn{13}{|l|}{ Mediator - Parents } \\
\hline SOFTA Total & - & - & - & - & - & - & - & - & - & - & 1.07 & $1.01-1.13$ \\
\hline SOFTA Engagement & - & - & - & - & - & - & - & - & - & - & 1.17 & $.97-1.40$ \\
\hline SOFTA Connection & - & - & - & - & - & - & - & - & - & - & 1.32 & $1.09-1.58$ \\
\hline SOFTA Safety & - & - & - & - & - & - & - & - & - & - & 1.24 & $1.02-1.50$ \\
\hline SOFTA Purpose & - & - & - & - & - & - & - & - & - & - & 1.07 & $.90-1.26$ \\
\hline
\end{tabular}


Note. All analyses included the following covariates: age, percentage median BMI at baseline, self-reported eating disorder pathology, duration of illness and site. Abbreviations: CI, Confidence Interval; OR, Odds Ratio; ASQ, Attachment Style Questionnaire; ASQ Discomfort, ASQ Discomfort with Closeness; ASQ Preoccupation, ASQ Preoccupation with Relationships; ASQ Secondary, ASQ Relationships as Secondary; DERS, Difficulties in Emotion Regulation Strategies Scale; DERS Non-acceptance, DERS Non-Acceptance of Emotional Responses; DERS Goals, DERS Difficulties Engaging in Goal-Directed Behaviors; DERS Impulse, DERS Impulse Control Difficulties; DERS Awareness, DERS Lack of Emotional Awareness; DERS Strategies, DERS Limited Access to Effective Emotion Regulation Strategies; DERS Clarity, DERS Lack of Emotional Clarity; RFQ8, Reflective Function Questionnaire (8 item version); RFQ8 Certainty, RFQ8 Certainty About Mental States; RFQ8 Uncertainty, RFQ8 Uncertainty About Mental States; HMZ, Hypermentalizing Questionnaire; SOFTA, System for Observing Family Therapy Alliance; SOFTA Engagement, SOFTA Engagement in the Therapeutic Process; SOFTA Connection, SOFTA Emotional Connection to the Therapist; SOFTA Safety, SOFTA Safety within the Therapeutic System; SOFTA Purpose, SOFTA Shared Sense of Purpose within the Family. 
Appendix K: Associations between adolescent exposure, mediator and outcome variables

\begin{tabular}{|c|c|c|c|c|c|c|c|c|c|c|c|c|}
\hline \multirow{3}{*}{$\begin{array}{l}\text { Predictors at baseline } \\
\text { Exposure - } \\
\text { Adolescents }\end{array}$} & \multicolumn{10}{|c|}{ Adolescent Mediator T2 } & \multirow{2}{*}{\multicolumn{2}{|c|}{$\begin{array}{l}\begin{array}{l}\text { Outcome at end of } \\
\text { treatment }\end{array} \\
\text { Morgan Russell } \\
\text { Outcome }\end{array}$}} \\
\hline & \multicolumn{2}{|c|}{ SOFTA Total } & \multicolumn{2}{|c|}{ SOFTA Engagement } & \multicolumn{2}{|c|}{ SOFTA Connection } & \multicolumn{2}{|c|}{ SOFTA Safety } & \multicolumn{2}{|c|}{ SOFTA Purpose } & & \\
\hline & Beta & $95 \% \mathrm{CI}$ & Beta & $95 \% \mathrm{CI}$ & Beta & $95 \% \mathrm{CI}$ & Beta & $95 \% \mathrm{CI}$ & Beta & $95 \% \mathrm{CI}$ & OR & $95 \% \mathrm{CI}$ \\
\hline$\overline{\text { ASQ Confidence }}$ & .41 & $.06-.75$ & .14 & $.04-.24$ & .06 & $-.06-.17$ & .13 & $.03-.24$ & .08 & $-.00-.16$ & 1.00 & $.93-1.07$ \\
\hline ASQ Discomfort & -.21 & $-.50-.09$ & -.08 & $-.16-.00$ & -.03 & $-.13-.07$ & -.07 & $-.16-.02$ & -.03 & $-.10-.03$ & 1.00 & $.95-1.06$ \\
\hline ASQ Secondary & -.31 & $-.76-.13$ & -.10 & $-.22-.02$ & -.08 & $-.22-.06$ & -.09 & $-.22-.05$ & -.04 & $-.15-.06$ & .99 & $.92-1.08$ \\
\hline ASQ Need for Approval . & .10 & $-.37-.57$ & .01 & $-.11-.14$ & .14 & $-.01-.28$ & -.05 & $-.19-.08$ & .01 & $-.11-.12$ & 1.00 & $.92-1.09$ \\
\hline ASQ Preoccupation & -.05 & $-.47-.38$ & -.03 & $-.15-.09$ & .06 & $-.08-.19$ & -.01 & $-.13-.12$ & -.07 & $-.18-.03$ & 1.01 & $.94-1.10$ \\
\hline DERS Total & -.05 & $-.15-.04$ & -.02 & $-.04-.01$ & .00 & $-.03-.04$ & -.03 & $-.06-.00$ & -.01 & $-.04-.01$ & 1.00 & $.98-1.02$ \\
\hline DERS Nonacceptance . & .10 & $-.25-.45$ & .03 & $-.07-.12$ & .07 & $-.04-.18$ & -.01 & $-.12-.09$ & .02 & $-.07-.11$ & 1.02 & $.95-1.09$ \\
\hline DERS Goals & .05 & $-.48-.58$ & -.01 & $-.15-.14$ & .08 & $-.08-.25$ & -.03 & $-.19-.13$ & .01 & $-.13-.14$ & .96 & $.88-1.06$ \\
\hline DERS Impulse & -.18 & $-.57-.20$ & -.07 & $-.18-.04$ & .02 & $-.09-.14$ & -.10 & $-.21-.02$ & -.04 & $-.14-.05$ & 1.01 & $.94-1.09$ \\
\hline DERS Awareness & -.72 & $-1.21--.23$ & -.22 & $-.35--.08$ & -.19 & $-.35--.03$ & -.16 & $-.31--.02$ & -.15 & $-.28--.02$ & 1.01 & $.92-1.11$ \\
\hline DERS Strategies & -.21 & $-.53-.11$ & -.06 & $-.14-.03$ & .01 & $-.10-.11$ & -.10 & $-.20-.00$ & -.06 & $-.14-.02$ & .97 & $.91-1.03$ \\
\hline DERS Clarity & -.15 & $-.68-.37$ & -.05 & $-.19-.10$ & .03 & $-.14-.20$ & -.07 & $-.23-.09$ & -.06 & $-.19-.06$ & 1.10 & $1.00-1.21$ \\
\hline HMZ Total & -.07 & $-.23-.08$ & -.03 & $-.07-.02$ & .01 & $-.04-.06$ & -.04 & $-.09-.00$ & -.02 & $-.06-.02$ & 1.01 & $.99-1.04$ \\
\hline RFQY & 4.50 & $1.10-7.90$ & 1.10 & $.14-2.07$ & 1.33 & $.19-2.47$ & 1.05 & $.02-2.07$ & 1.02 & $.19-1.85$ & 1.15 & $.68-1.94$ \\
\hline \multicolumn{13}{|l|}{ Mediator - Adolescents } \\
\hline SOFTA Total & - & - & - & - & - & - & - & - & - & - & 1.04 & $.99-1.08$ \\
\hline SOFTA Engagement & - & - & - & - & - & - & - & - & - & - & 1.11 & $.96-1.27$ \\
\hline SOFTA Connection & - & - & - & - & - & - & - & - & - & - & 1.09 & $.96-1.24$ \\
\hline SOFTA Safety & - & - & - & - & - & - & - & - & - & - & 1.08 & $.93-1.26$ \\
\hline SOFTA Purpose & - & - & - & - & - & - & - & - & - & - & 1.18 & $1.01-1.38$ \\
\hline
\end{tabular}

Note. All analyses included the following covariates: age, percentage median BMI at baseline, self-reported eating disorder pathology, duration of illness and site. 
Abbreviations: CI, Confidence Interval; OR, Odds Ratio; ASQ, Attachment Style Questionnaire; ASQ Discomfort, ASQ Discomfort with Closeness; ASQ Preoccupation, ASQ Preoccupation with Relationships; ASQ Secondary, ASQ Relationships as Secondary; DERS, Difficulties in Emotion Regulation Strategies Scale; DERS Nonacceptance, DERS Nonacceptance of Emotional Responses; DERS Goals, DERS Difficulties Engaging in Goal-Directed Behaviors; DERS Impulse, DERS Impulse Control Difficulties; DERS Awareness, DERS Lack of Emotional Awareness; DERS Strategies, DERS Limited Access to Effective Emotion Regulation Strategies; DERS Clarity, DERS Lack of Emotional Clarity; RFQY, Reflective Function Questionnaire - Youth Total Score; HMZ, Hypermentalizing Questionnaire; SOFTA, System for Observing Family Therapy Alliance; SOFTA Engagement, SOFTA Engagement in the Therapeutic Process; SOFTA Connection, SOFTA Emotional Connection to the Therapist; SOFTA Safety, SOFTA Safety within the Therapeutic System; SOFTA Purpose, SOFTA Shared Sense of Purpose within the Family. 